\title{
Volatile communication between fungi and bacteria
}

Ruth Lydia Schmidt 


\section{Thesis committee}

\section{Promotor}

Prof. Dr W. de Boer

Special professor Microbial Soil Ecology

Wageningen University \& Research

\section{Co-promotor}

Dr P Garbeva,

Senior researcher, Netherlands Institute of Ecology (NIOO-KNAW), Wageningen

\section{Other members}

Prof. Dr B.P.H.J. Thomma, Wageningen University \& Research

Prof. Dr M.E. Maffei, University of Turin, Italy

Prof. Dr L. Weisskopf, University of Fribourg, Switzerland

Dr A. Deveau, French National Institute for Agricultural Research (INRA), France

This PhD research was conducted under the auspices of the C.T. de Wit Graduate School for Production Ecology \& Resource Conservation (PE\&RC) 


\title{
Volatile communication between fungi and bacteria
}

\author{
Ruth Lydia Schmidt
}

\section{Thesis}

submitted in fulfillment of the requirements for the degree of doctor at Wageningen University

by the authority of the Rector Magnificus

Prof. Dr A. P. J. Mol,

in the presence of the

Thesis Committee appointed by the Academic Board to be defended in public

on Friday 20th of October 2017

at 1:30 p.m. in the Aula. 
Ruth L. Schmidt

Volatile communication between fungi and bacteria 222 pages.

PhD thesis, Wageningen University, Wageningen, the Netherlands (2017) With references, with summaries in English and German

ISBN: 978-94-6343-688-5

DOI: https://doi.org/10.18174/422840 
"Nothing in life is to be feared. It is only to be understood." Marie Curie 



\section{Table of Contents}

Abstract Why smell is so important for life on earth $\quad 9$

Chapter 1 General introduction and thesis outline $\mathbf{1 1}$

$\begin{array}{lll}\text { Chapter } 2 \text { Volatile affairs in microbial interactions } & \mathbf{2 1}\end{array}$

Chapter $\mathbf{3}$ Microbial small talk: Volatiles in fungal-bacterial interactions $\mathbf{3 5}$

Chapter 4 Fungal volatile compounds induce production of the secondary metabolite Sodorifen in Serratia plymuthica PRI-2C

Chapter 5 Exploring the genomic traits of fungus-feeding bacterial genus Collimonas

Chapter 6 Deciphering the Fusarium culmorum genome:

from genes to products

145

Chapter 7 General discussion

References

Summary

Zusammenfassung 209

Acknowledgement

Curriculum vitae 



\section{Abstract}

\section{Why smell is so important for life on earth}

If you are small, smells are a good way to stand out. Soil microorganisms, such as bacteria and fungi, produce an array of pleasant or repelling odors, also known as volatile compounds. These compounds can easily travel through the abundant air- and water-filled pockets of the soil, mediating interaction and communication amongst physically separated microorganisms. Hence, volatiles play important roles in the microbial world. In this PhD thesis I explored how bacteria and fungi use volatiles to communicate with each other and studied the underlying mechanisms of such volatile dialogues. Fungal volatiles, in particular terpenes, showed to affect the ability of bacteria to move. But not only that; being exposed to a terpeneproducing plant-pathogenic fungus, a beneficial bacterium started to produce its own terpenes. It is still unclear how often these dialogues take place with other microorganisms and plants. But since there are billions of other microorganisms, of which many have the genetic repertoire to produce terpenes, it seems likely that "terpene" is the most spoken language belowground. Thus, this thesis sets the basis for future research on microbe-microbe and plant-microbe interactions and, by understanding their ecological roles, for developments towards sustainable solutions for crop welfare. 



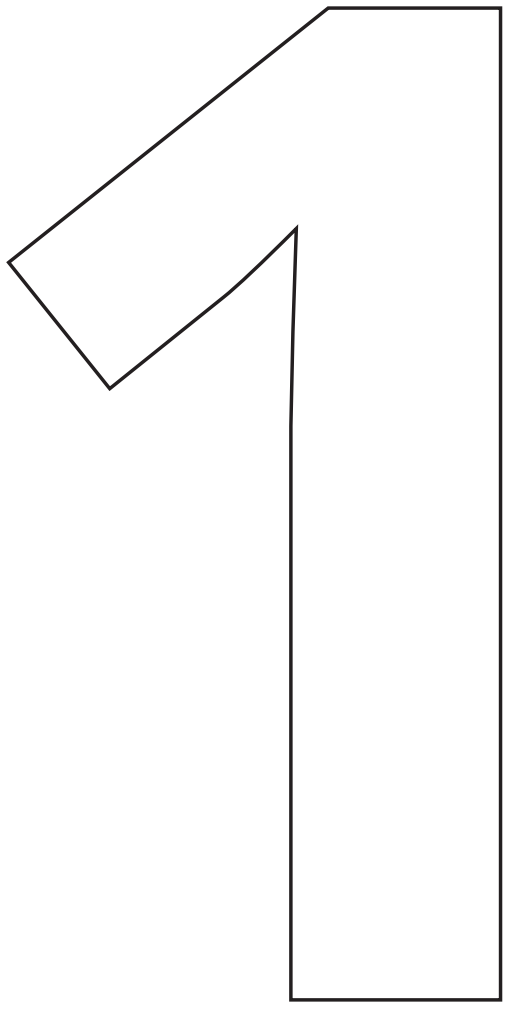

General introduction and thesis outline 

The sense of smell evokes the strongest memory of all the senses. As Rachel Carson writes: "The sense of smell, almost more than any other, has the power to recall memories..." How does this relate to microorganisms? Most odors we perceive as humans are in fact produced by microorganisms. Imagine taking a walk in a forest after the first rainfall after a dry spell, and it hits you: the sweet, fresh, powerfully evocative smell of fresh rain. This earthy-smelling substance is geosmin, a chemical released into the air by soil-dwelling bacteria when rain falls onto dry soil. Or think of the characteristic seashore aroma, which is due to dimethyl sulfide, a rather stinky sulfur compound that puts the funk in everything from nori, to truffles, and beer. But not all bacterial odors are necessarily good in the perspective of the receiver; especially sulfur compounds can be repelling, just think of the smell of rotten eggs or potatoes.

Odors, good and bad, are due to volatile organic compounds (VOCs), and can be a form of information signals between different organisms. Chemically speaking, VOCs are small carbon-containing molecules with low molecular weight, high vapour pressure, and low boiling point (Insam \& Seewald, 2010, Effmert et al., 2012). In environments, where microbes are physically separated and rely on communicating with each other, one could think of VOCs as "words" that build the "language" of microorganisms. This form of language has been already described in plants as means of attractants and communication signals with other plants and belowground organisms to "cry for help" when under attack (Dicke et al., 1990). Although previous work has shown that microbial VOCs are capable of inhibiting or stimulating other individuals (Stotzky \& Schenck, 1976), it was only in the last two decades that the interest in microbial VOCs experienced a revival (Garbeva et al., 2011, Bailly \& Weisskopf, 2012, Effmert et al., 2012, Schmidt et al., 2015). At this moment we are just in the beginning to decipher the versatile ecological roles of VOCs in microbial interactions. The knowledge we have gained from research so far shows that VOCs can have both beneficial and harmful effects towards the interacting organism, ranging from growth promotion to growth inhibitory effects (Effmert et al., 2012, Schmidt et al., 2015). In the following, I will explore the variety of microbial aromas and their biosynthesis and zoom into the complexity of VOCs in microbial interactions. 


\section{How many microbial aromas are there and how are they made?}

Taking into consideration that the Earth is predicted to be home to upward of 1 trillion (1012) microbial species (Locey \& Lennon, 2016), the aroma or VOC profiles of only a small number of microorganisms are known. Up to date, more than 1000 VOCs of bacterial and fungal origin have been described in literature (Effmert et al., 2012). Microbial VOCs are usually composed of alkenes, alcohols, aldehydes, ketones, terpenes, benzenoids, pyrazines, acids, and esters (Piechulla \& Degenhardt, 2014) and derive from the microbes' primary and secondary metabolism (Korpi et al., 2009). The biosynthetic pathways involved in the production of VOCs include aerobic, heterotrophic carbon metabolism, fermentation, amino-acid catabolism, terpenoid biosynthesis, fatty acid degradation and sulfur reduction (Penuelas et al., 2014). Particularly, the group of terpenes represents an interesting group of VOCs when studying microbial interactions. These compounds have been shown to play a variety of roles in mediating antagonistic and beneficial interactions among microorganisms (Gershenzon \& Dudareva, 2007, Dickschat et al., 2014).

Terpenes represent the largest class of VOCs with over 50,000 known members, including monoterpenes, sesquiterpenes, and diterpenes (Hill, 1991, Dickschat et al., 2014). Despite their remarkable chemical and functional diversity, the biosynthesis of all terpenes starts from just a few acyclic precursors, including geranyl diphosphate (GPP) farnesyl diphosphate (FPP) and geranylgeranly diphosphate (GGPP) (Dickschat et al., 2014). The primary enzymes responsible for catalyzing the formation of monoterpenes, sesquiterpenes or diterpenes are terpene synthases (Tholl, 2006). Even though terpenes are generally considered to be plant metabolites, recent research shows that microorganisms are a rich source of terpenes too (Dickschat et al., 2014). An increasing number of terpenes have been reported for fungi, most of them being sesquiterpenes (Collado et al., 2007, Ebel, 2010, Dickschat, 2017). Even more recently, the growing knowledge on bacterial genomes resulted in the discovery of terpenes and their synthases in bacteria, indicating that the genetic capacity of bacteria to make terpenes is widespread (Cane \& Ikeda, 2012, Song et al., 2015). Hence, it is an exciting era to study terpenes as means of communication signals between microorganisms. 


\section{Volatiles in fungal-bacterial interactions}

Bacteria together with fungi make up the two major groups of microorganisms inhabiting all ecosystems on our planet, including the soil (Prosser, 2015). However, the knowledge on fungal-bacterial interactions is still limited. One such way of interaction that has been vastly overlooked is mediated by VOCs. VOC with inhibitory effects on fungi, a phenomenon called fungistasis, is already known since the 70s (Hora \& Baker, 1970, Hora \& Baker, 1972, Lockwood, 1977). However, only in recent years, several studies paid renewed attention to the fungistatic effect of VOCs on spore germination and mycelial growth for a variety of fungi, including plant pathogenic fungi (Wheatley, 2002, Vespermann et al., 2007, Zou et al., 2007, Kai et al., 2009, Garbeva et al., 2011). Such VOCs with antifungal activity often include sulphur compounds, like dimethyl disulfide, dimethyl trisulfide, and S-methyl thioacetate and benzonitrile (Garbeva et al., 2014). Besides these effects, several bacterial VOCs have been reported to modify fungal morphology, enzyme activity and gene expression (Mackie \& Wheatley, 1999, Minerdi et al., 2008, Minerdi et al., 2009, Garbeva et al., 2014). Bacterial VOCs have been shown to also affect the growth of other bacteria, induce changes in motility and antibiotic resistance (Bitas et al., 2013, Garbeva et al., 2014, Hagai et al., 2014).

Already in 1966, Dick and Hutchinson indicated that VOCs mediate interactions between fungi. However, up till now most research has been focused on bacterial VOCs and only little is known about fungal strategies to employ VOCs to contact or combat other fungi or bacteria (Werner et al., 2016). Despite this lack of knowledge, probably most of us know the most common fungal VOC 1-Octen3-ol that is also known as "mushroom alcohol" and is essential for the earthy, "mushroomy" aroma and flavour of fungi (Morath et al., 2012). This compound has been described to act on Penicillium species by inhibition of mycelial growth and suppression of conidia germination (Werner et al., 2016). The broad range of VOC profiles emitted by members of the genus Trichoderma is the reason for its extensive use as biocontrol agents in agriculture to fight against the fungal pathogens.

Similar to fungistasis, bacteriostasis describes the inhibition of bacterial growth. Few studies have described fungal VOCs with bacteriostatic effects. For example, VOCs produced by the oyster mushroom Pleurotus ostreatus showed inhibitory effects on Bacillus cereus and Bacillus subtilis (Beltran-Garcia et al., 1997). 
Another study demonstrated that VOCs emitted by $T$. atroviride increased the expression of a biocontrol gene (ph|A) of $P$. fluorescens (Lutz et al., 2004).

These examples suggest that both, fungi and bacteria employ VOCs to influence their target organism in various ways.

\section{Volatiles and the environment soil}

The soil and the rhizosphere represent dynamic environments for fungi and bacteria to thrive. Especially the rhizosphere, the soil compartment surrounding and influenced by the plant roots, represents a hotspot for microbial life due to the high amount of organic nutrients (in form of root exudates) released by the roots. By taking up those nutrients microbes interact in various ways, ranging from competition to cooperation, which in turn has an effect on the health of the plant (Mendes et al., 2013). These interactions are mediated by a range of secondary metabolites produced by the microorganisms. Especially in long-distance interactions, the unique properties of VOCs are ideal compounds to travel through the abundant air- and water-filled pockets in the soil (Berendsen et al., 2012). Besides VOCs, soluble compounds such as antibiotics and siderophores as well as enzymes play important roles in direct interactions between microorganisms (Insam \& Seewald, 2010).

The production of VOCs in soil is influenced by various factors, including the growth stage, nutrient availability, temperature, oxygen availability, $\mathrm{pH}$, and moisture content (Wheatley, 2002, Insam \& Seewald, 2010). Thus, when studying microbial interactions in natural environments it is of crucial importance to consider representative conditions.

\section{Conclusions}

The importance of VOCs as molecules in long-distance microbial interactions is becoming apparent. Despite the growing body of literature supporting this, the ecological roles of and underlying mechanisms of VOC-mediated microbial interactions still remain largely unknown. New detection techniques will allow to characterise the "volatilome" involved in such interactions. Moreoever, combining state-of-the-art "omic" tools such as genomics, transcriptomics and proteomics will give insight into underlying molecular mechanisms of VOC perception and responses. 


\section{Major questions}

What are the ecological roles of microbial VOCs?

Can bacteria sense and respond to fungal VOCs?

Do bacteria react with specific phenotypic responses to fungal VOCs?

Can nutrient condition and fungal growth stage effect VOCs mediated interactions?

How are VOCs perceived by the interacting microorganism and which regulatory genes and pathways are involved in the response?

Do terpenes form major constituents of a common language between fungi and bacteria?

\section{Research questions and thesis outline}

The major aim of this thesis was to zoom into the complexity of the volatile-mediated fungal bacterial interactions and to investigate the underlying mechanisms. For this, in vitro bioassays were combined with several "omic" approaches including, genomics, metabolomics, transcriptomics and proteomics (Fig. 1). 


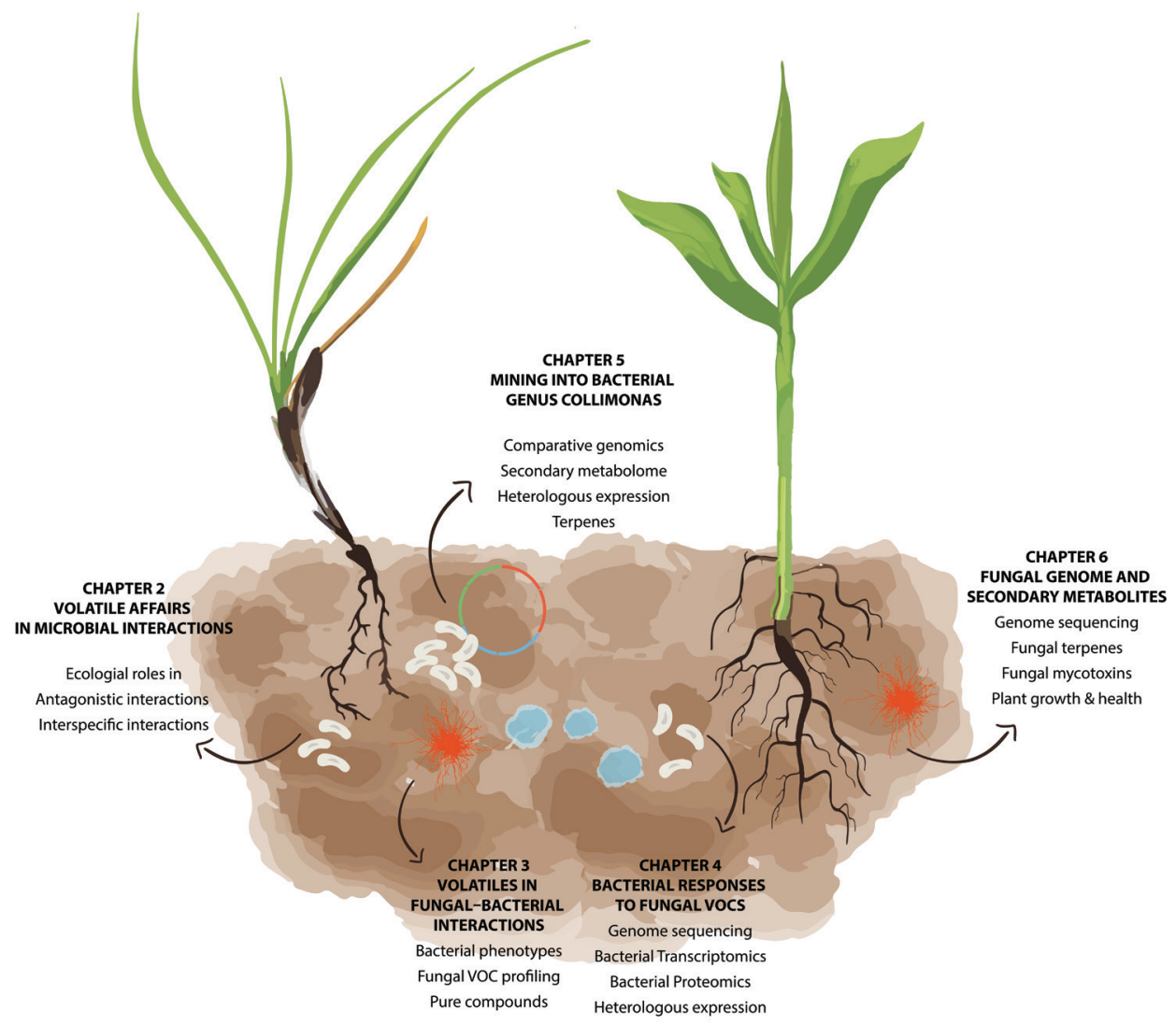

Fig. 1 / Schematic overview of the chapters of this thesis

Microbial VOCs and their various effects on other organisms have been receiving increased attention in the last few decades. However, their ecological roles still remain largely unknown. With the question "What are the ecological roles of VOCs in microbe-microbe interactions?" in Chapter $\mathbf{2}$ we will provide an up-todate review of the activities and the natural roles of VOCs in microbial interactions, including their antibacterial and antifungal activities, the possible modes of action and their effects on pathogen growth, morphology, enzyme activity, gene expression. Furthermore, the chapter highlights the current challenges in studying microbial VOCs and their future applications.

Based on the hypothesis that VOCs play important roles in the communication between microbes, I asked whether "Soil bacteria are able to sense fungal vocs 
and in response modify their behaviour?" In Chapter $\mathbf{3}$ I demonstrate that VOCS produced by fungi/oomycetes can be sensed by bacteria, and therefore modulate their behaviour (e.g. motility). These results shed new lights into one possible mechanism used by particular soil and rhizospheric fungi/oomycetes to attract or repel bacterial neighbours.

Inspired by the findings that bacteria change their ability to move in response to fungal VOCS, I asked "What are the molecular mechanisms underlying bacterial responses to fungal voCs?" In Chapter $\mathbf{4}$ I describe the discovery of several genes and proteins involved in the mechanisms underlying the various responses to fungal VOCs. Moreover, I identified the specific terpene molecule sodorifen as one of the key player in the fungal-bacterial dialogue.

To identify genes potentially playing a role in VOC and secondary metabolite production, I asked "Which gene clusters are involved in the production of vocs and other secondary metabolites?" In Chapter $\mathbf{5}$ we selected Collimonas as model organism to mine into several genomes and to search for genes encoding for secondary metabolites with potential relevance for interactions with fungi and other microorganisms. Here we identified several genes encoding for novel enzymes and secondary metabolites (including terpenes), which reflect the behavior, antimicrobial activity and mycophagous lifestyles of Collimonas spp.

Linking genes to their biosynthetic pathways for VOC production, in particular terpenes, is still a missing corner piece in VOC research. Thus, in Chapter 6, I address the question "Which genes are linked to the production of fungal terpenes?" by exploring the genome of the fungal plant pathogen Fusarium culmorum for genes and their related products. I describe several terpene synthases, their biosynthetic pathways, and related products as well as mycotoxins that play important roles in fungal pathogenicity and fungal-bacterial interactions.

In Chapter $\mathbf{7}$ I integrate the findings of this thesis and further discuss the role of VOCs in microbial interactions. Furthermore, I discuss the importance of VOCs mediated interactions and the potential future applications of microbial VOCs. 
20 / Chapter 1 


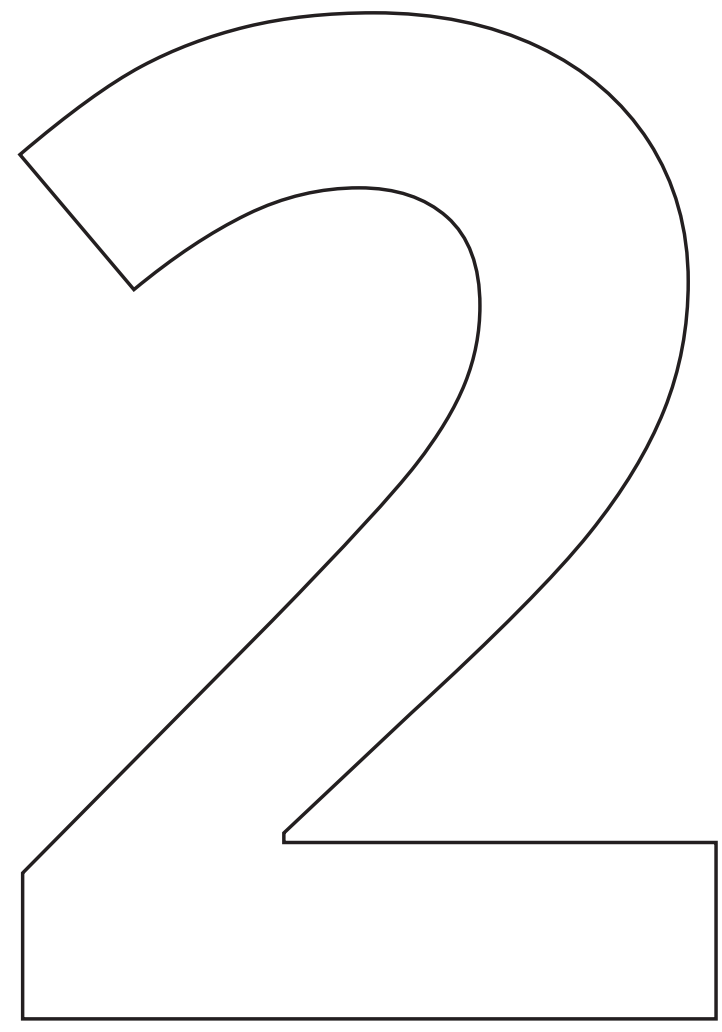

\section{Volatile affairs in microbial interactions}

Ruth Schmidt*, Viviane Cordovez*, Wietse de Boer, Jos Raaijmakers and Paolina Garbeva

*Authors contributed equally to this work

The ISME Journal (2015) 



\section{Abstract}

Microorganisms are important factors in shaping our environment. One key characteristic that has been neglected for a long time is the ability of microorganisms to release chemically diverse volatile compounds. At present, it is clear that the blend of volatiles released by microorganisms can be very complex and often includes many unknown compounds for which the chemical structures remain to be elucidated. The biggest challenge now is to unravel the biological and ecological functions of these microbial volatiles. There is increasing evidence that microbial volatiles can act as info-chemicals in interactions among microbes and between microbes and their eukaryotic hosts. Here, we review and discuss recent advances in understanding the natural roles of volatiles in microbe-microbe interactions. Specific emphasis will be given to the antimicrobial activities of microbial volatiles and their effects on bacterial quorum sensing, motility, gene expression and antibiotic resistance. 


\section{Introduction}

Microorganisms from diverse ecosystems produce a wide range of volatile organic compounds. Compared with other secondary metabolites (for example, enzymes, antibiotics and toxins), volatiles are typically small compounds (up to C20) with low molecular mass (100-500 Daltons), high vapour pressure, low boiling point and a lipophilic moiety. These properties facilitate evaporation and diffusion through both water- and gas-filled pores in soil and rhizosphere environments. Hence, microbial volatiles have important roles in marine and terrestrial environments (Schulz \& Dickschat, 2007, Romoli et al., 2014). To date, the chemical structure of 1000 volatiles have been described originating from a wide range of bacterial and fungal genera and species (Effmert et al., 2012, Lemfack et al., 2014). Bacterial volatiles are typically dominated by alkenes, alcohols, ketones, terpenes, benzenoids, pyrazines, acids and esters, whereas fungal volatiles are dominated by alcohols, benzenoids, aldehydes, alkenes, acids, esters and ketones (Piechulla \& Degenhardt, 2014). Most microbial volatiles are considered as side-products of primary and secondary metabolism. They are formed mainly by oxidation of glucose from various intermediates (Korpi et al., 2009). The underlying biosynthetic pathways are aerobic, heterotrophic carbon metabolism, fermentation, amino-acid catabolism, terpenoid biosynthesis, fatty acid degradation and sulphur reduction (Penuelas et al., 2014). The main metabolic pathways for microbial volatiles are summarized in Fig. 1. 


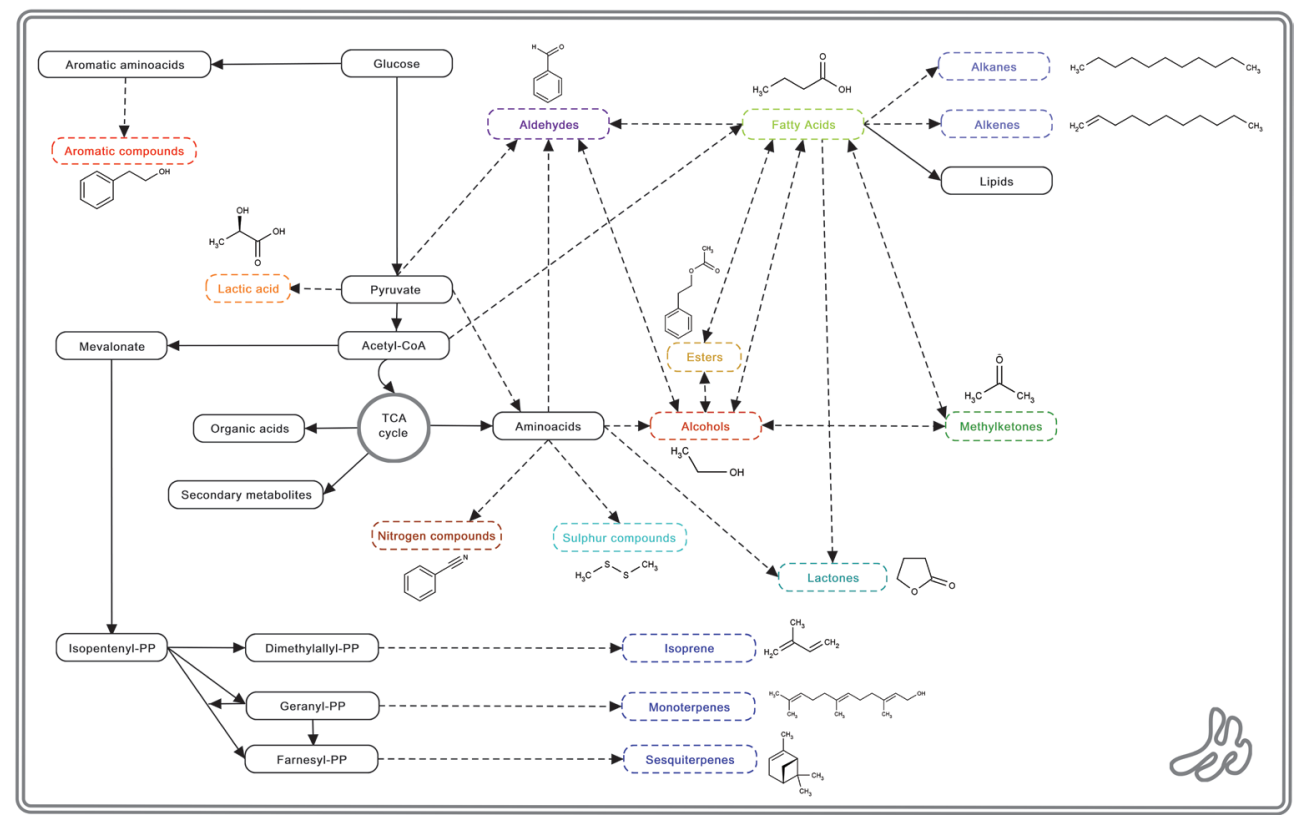

Fig. 1 / Main metabolic pathways for the production of microbial volatiles. Volatiles are depicted in colored dashed rectangles indicating different chemical classes. Representative examples are given per class: alcohols (for example, ethanol), aldehydes (for example, benzaldehyde), alkanes (for example, undecane), alkenes (1-undecene), aromatic compounds (for example, 2-phenylethanol), esters (for example, 2-phenylethyl ester), fatty acids (for example, butyric acid), isoprene, lactic acid, lactones (for example, gamma-butyrolactone), methylketones (for example, acetone), monoterpenes (for example, farnesol), nitrogen compounds (for example, benzonitrile), sesquiterpenes (for example, pinene) and sulphur compounds (for example, dimethyl disulphide).

Although there are common volatiles produced by different, often unrelated, microorganisms, other volatiles are unique for certain strains (Schulz \& Dickschat, 2007, Garbeva et al., 2014, Garbeva et al., 2014). The amount and composition of volatiles produced by microorganisms can vary according to culturing conditions (Claeson et al., 2007, Blom et al., 2011, Garbeva et al., 2014, Garbeva et al., 2014). Other important factors influencing the production of volatiles are the physiological state of the producing microorganism, oxygen availability, moisture, temperature and pH (Insam \& Seewald, 2010, Romoli et al., 2014).

The importance of microbial volatiles for the ecology of microorganisms has been overlooked for a long time, probably due the lack of appropriate detection techniques. However, in the last 10 years the number of studies on microbial 
volatiles has increased substantially in different research areas such as food, medical, agricultural and environmental sciences. In this review, we focus on the ecological role of volatiles in microbe-microbe interactions. For more information on techniques used for volatile analyses and their role in microbe interactions with their eukaryotic hosts, we refer to several recent reviews (Effmert et al., 2012, Farag et al., 2013, Junker \& Tholl, 2013, Penuelas et al., 2014).

\section{Ecological roles of microbial volatiles in antagonistic interactions}

Microbial volatiles can have a significant role in antagonistic interactions between microorganisms occupying the same ecological niche. Here, we will focus on the antimicrobial activity of volatiles with specific emphasis on their antifungal and antibacterial activities.

\section{Volatile-mediated antifungal activity}

It is well known that germination of fungal spores as well as hyphal growth can be inhibited by bacterial volatiles (Herrington et al., 1985, Herrington et al., 1987). Furthermore, exposure to bacterial volatiles has been reported to change fungal morphology, enzyme activity and gene expression (Wheatley, 2002, Vespermann et al., 2007, Minerdi et al., 2008, Kai et al., 2009, Minerdi et al., 2009, Garbeva et al., 2011, Garbeva et al., 2014). For example, activity of laccases and tyrosinases can be strongly affected by bacterial volatiles (Wheatley, 2002).

Fungal volatiles can also have inhibitory effects on other fungi. For example, the endophytic fungi Muscodor albus and Oxysporus latemarginatus strongly inhibited growth of several plant pathogenic fungi, including Botrytis cinerea and Rhizoctonia solani (Strobel et al., 2001). Moreover, M. albus volatiles were shown to kill the fungal human pathogens Aspergillus fumigatus and Candida albicans (Strobel et al., 2001). Fungi often live in symbiosis with bacteria. For Fusarium oxysporum, hyphae-associated bacteria were shown to produce the volatile sesquiterpene caryophyllene, which repressed the expression of two virulence genes. When cured from the bacterial symbionts, caryophyllene was not detected and F. oxysporum became pathogenic (Minerdi et al., 2008).

Sensitivity to volatiles can strongly differ between fungal species and the extent of inhibition depends on the individual bacteria-fungus or fungus-fungus interaction (Kai et al., 2007, Vespermann et al., 2007, Kai et al., 2009, Garbeva et 
al., 2014). Several independent studies have reported that $F$. solani is not much affected by bacterial volatiles, whereas Pythium species (oomycetes) are highly sensitive to bacterial volatiles (Kai et al., 2009, Effmert et al., 2012, Garbeva et al., 2014, Garbeva et al., 2014). F. oxysporum was also reported to be rather resistant to volatiles produced by the fungus O. latemarginatus, whereas Magnaporthe grisea was sensitive. High sensitivity to bacterial volatiles was recently reported for the late blight oomycete pathogen Phytophthora infestans. Two volatiles, hydrogen cyanide and 1 -undecen, were indicated as the main compounds responsible for the growth inhibition (Hunziker et al., 2015). The apparent high sensitivity of oomycetes to volatiles may be related to their cell wall composition and structure, which is different from that of fungi. To date, however, very little is known about fungal resistance to volatiles with the exception of resistance to azole-derived compounds (Lupetti et al., 2002). Azole resistance commonly involves modifications of the cyp51A gene, the target of antifungal azoles (Lupetti et al., 2002, Seyedmousavi et al., 2014). The resistance selection is believed to occur via exposure to azole compounds in the environment (Snelders et al., 2009), released by humans via application of crop protections agents or by bacterial genera commonly found in soil, such as Bacillus, Serratia, Pseudomonas and Burkholderia (Lemfack et al., 2014).

\section{Volatile-mediated antibacterial activity}

Relatively fewstudies have reported on volatiles with antibacterial activity. Screenings of commonly produced volatiles with antimicrobial activity often did not reveal antibacterial activity (Schulz et al., 2010). Moreover, volatiles with strong antifungal activity (such as dimethyl disulphide, dimethyl trisulphide, S-methyl thioacetate and benzonitrile) did not exhibit antibacterial effects and even stimulated the growth of some bacteria (Garbeva et al., 2014). However, some specific volatiles produced by only a few microorganisms have been indicated as potential antibacterial agents. These include volatile lactones such as y-butyrolactones, which exhibit antibacterial activity against a broad range of Gram-positive and Gram-negative bacteria (Schulz et al., 2010).

An odoriferous actinomycete isolate from corn seeds, identified as Streptomyces albidoflavus, was shown to produce a sesquiterpene, named albaflavenone with antibacterial properties (Gurtler et al., 1994). More recently, albaflavenone was isolated from other Streptomyces species and fungi (Takamatsu et al., 2011, Moody et al., 2012). Another sesquiterpene compound with antibacterial 
activity, dihydro- $\beta$-agarofuran, is produced by Streptomyces sp. (Brana et al., 2014). Recently, Dandurishvili et al. (2011) reported that volatiles emitted by Pseudomonas fluorescens and Serratia plymuthica have bacteriostatic effects against the bacterial plant pathogens Agrobacterium tumefaciens and A. vitis and inhibited the growth of these pathogens in planta. The major volatile emitted by S. plymuthica under the tested conditions was dimethyl disulphide, whereas $P$. fluorescens emitted a mix of 1-undecene, methanthiol, methanthiol acetate and dimethyl disulphide (Dandurishvili et al., 2011).

Volatile-producing endophytes have recently attracted great attention due to their strong antimicrobial activity. For example, M. albus (an endophytic fungus of tropical tree species) emitted a number of volatiles, such as tetrohydofuran, aciphyllene and an azulene derivate (Atmosukarto et al., 2005). Volatiles emitted by $M$. albus as well as the artificial mixture of volatiles effectively inhibited or killed a range of plant and human-pathogenic bacteria. Another recently described endophytic fungus M. crispans, isolated from wild pineapple, produced a mixture of volatile compounds with strong activity against a major bacterial pathogen of citrus, Xanthomonas axonopodis pv. citri, and the human pathogens Yersinia pestis, Mycobacterium tuberculosis and Staphylococcus aureus (Mitchell et al., 2010). Five classes of volatiles (acids, alcohols, esters, ketones and lipids) were identified in Muscodor species and although each class had some antimicrobial effect, their collective action was required to kill a broad range of bacterial pathogens.

In the past years, a group of pyrazine volatile compounds have been attracting wide interest due to their promising antitumour, antimicrobial and insecticidal activities (Rajini et al., 2011). The production of pyrazines is widely distributed in plants, and only few bacteria have been reported so far to synthesize these volatile compounds (Rajini et al., 2011, Brana et al., 2014). S. albus, Corynebacterium g/utamicum and Bacillus spp. produce tetramethylpyrazine (also known as ligustrazine), a compound that is used in traditional Chinese medicine against cystic fibrosis.

Although the mode of action of antibacterial volatiles has not been studied in detail, it is likely that hydrophobicity of some volatiles enables them to partition in the lipid layer of the cell membrane, rendering the membrane more permeable. Indeed, a study on the mechanisms of inhibitory action of three monoterpenes against S. aureus and Escherichia coli revealed a perturbation of the lipid fraction 
of microorganisms' plasma membrane, resulting in alteration of membrane permeability and a leakage of intracellular compounds (Trombetta et al., 2005).

Finally, volatile compounds may have a synergistic effect when combined with antibiotics. For example, hydrophilic antibiotics such as vancomycin and $\beta$-lactam antibiotics, which have a marginal activity on the Gram-negative bacteria $E$. coli and Listeria monocytogenes, exhibit an enhanced antibacterial activity when pretreated with the volatile eugenol (Hemaiswarya \& Doble, 2010). Synergistic effects of terpenes and penicillin on multiresistant strains S. aureus and E. coli have also been reported (Gallucci et al., 2009).

\section{Ecological role of microbial volatiles in interspecific interactions}

Volatiles have an important role in interactions between physically separated microorganisms. Microarray analysis of E. coli exposed to volatiles emitted by Bacillus subtilis revealed that volatiles induce changes in gene expression and affect motility and biofilm formation of the exposed bacteria (Bitas et al., 2013). More recently, a study, using P. putida as a model organism, showed that indole has a role as an interspecific signalling molecule (Molina-Santiago et al., 2014). This compound influenced the expression pattern of $P$. putida genes involved in cell metabolism, cell wall biosynthesis and stress defence. In our research group, we have tested the effect of volatiles emitted by different soil bacteria grown in sand supplemented with artificial root exudates on the soil bacterium $P$. fluorescens. The $P$. fluorescens strain was grown on nutrient-limited agar while being exposed to volatiles produced by four phylogenetically different bacterial isolates (Collimonas pratensis, S. plymuthica, Paenibacillus sp. and Pedobacter sp.) as well as a mixture of all four bacteria. A genome-wide, microarray-based analysis revealed that volatiles of each bacterial strain affected gene expression of $P$. fluorescens, but with a different pattern for each strain. Only a small core set of 22 genes was differentially expressed by all volatileproducing bacteria, including the mixture. These genes were mainly involved in amino-acid transport and metabolism, energy production and conversion, signal transduction mechanisms, inorganic ion transport and metabolism, secretion and cell motility. Among these common, differentially expressed genes was the Pfl_0064 catalase, an important enzyme that protects the cell against damage by reactive oxygen species (Lushchak, 2001). Furthermore, the volatiles produced by C. pratensis triggered the production of antimicrobial secondary metabolites (Garbeva et al., 2014). 
Antibiotic production triggered by volatiles in microbial interactions was also observed in $P$. aeruginosa during co-culture with Enterobacter aerogenes and this enhanced production was due to the volatile 2,3-butanediol emitted by E. aerogenes (Venkataraman et al., 2014). Also for Chromobacterium violaceum and P. aeruginosa, several monoterpenes increased violacein and pyocyanin production, respectively (Ahmad et al., 2015). The fact that the production of antibiotics in these bacteria is regulated by quorum sensing (QS) suggests that volatiles may interfere with bacterial cell-cell communication. Indeed, several studies revealed that volatiles can affect QS systems in bacteria, negatively or positively (Schulz et al., 2010, Chernin et al., 2011, Ahmad et al., 2015). For example, volatiles produced by S. plymuthica can inhibit cell-cell communication mediated by acyl homoserine lactone molecules in Agrobacterium, Pectobacterium and Pseudomonas. Volatiles emitted by S. plymuthica decreased the amount of acyl homoserine lactone produced by these bacteria, leading to significant suppression of transcription of acyl homoserine lactone synthase genes (Chernin et al., 2011).

Volatiles may also influence fungal QS as well as fungal development and virulence. C. albicans and C. dubliniensis, well-known human opportunistic pathogenic yeasts, produce large amounts of the QS molecule (E,E)-farnesol, a sesquiterpene, that is able to modulate morphogenesis of these species. Accumulation of farnesol blocked the yeast-to-mycelium morphology switch, mycelial development and biofilm formation, important traits for virulence of Candida (Hornby et al., 2001, Martins et al., 2007). Moreover, volatiles produced by Trichoderma were shown to function as signalling molecules regulating development and mediating intercolony communication: volatiles such as 1-octen-3-ol, 3-octanol and 3-octanone produced by conidiating colonies elicited conidiation in other colonies (Nemcovic et al., 2008). The underlying mechanisms of the effects volatiles on fungal development remain largely unknown.

Recently, several studies reported on the effect of volatiles on bacterial antibiotic resistance or tolerance. For example, exposure of $E$. coli to volatiles emitted by Burkholderia ambifaria increased its resistance to gentamycin and kanamycin by yet unknown mechanisms (Groenhagen et al., 2013). Exposure to the volatile compound trimethylamine was shown to modify the antibiotic resistance profiles of several Gram-positive and Gram-negative bacteria (Letoffe et al., 2014). In addition, indole, a volatile that has been proposed to act as signalling molecule, 
can also affect antibiotic resistance. For example, P. putida does not produce the volatile indole itself but recognizes indole produced by other bacteria (for example, E. coli) and activates the expression of the gene encoding the TtgGHI efflux pump (Lee et al., 2010, Molina-Santiago et al., 2014). Biogenic ammonia, an inorganic volatile compound, was also reported to modify antibiotic resistance in physically separated bacteria (Bernier et al., 2011). One of the underlying mechanisms proposed involves ammonia-induced synthesis of polyamines, which alters the permeability of the bacterial membrane or helps the bacteria to cope with oxygen radicals. A recent study reported on ammonia-mediated growth promotion of ampicillin-sensitive bacteria by means of antibiotic inactivation (Cepl et al., 2014). However, this phenomenon appeared to result from $\mathrm{pH}$ increase in the media caused by bacterial volatiles rather than by alteration of specific traits in the target bacterium. Another inorganic volatile compound, hydrogen sulphide, was suggested as a universal defence against antibiotics in bacteria as it seemed to trigger broadspectrum antibiotic resistance, most probably due to alleviation of oxidative stress (Shatalin et al., 2011).

As was shown for the above mentioned Candida species, also virulence and fitness of microorganisms can be affected by microbial volatiles. This was for instance observed for Pectobacterium species, bacterial pathogens responsible for soft rot disease in potato. Disruption of the biosynthesis of the volatile 2,3-butanediol coincided with reduced virulence (Marquez-Villavicencio Md et al., 2011).

Volatile compounds can also have a role in the attraction of other microorganisms. During interaction between $X$. perforans and Paenibacillus vortex, volatiles produced by $X$. perforans were found to attract the proficient swarmer $P$. vortex (Hagai et al., 2014). Interestingly, the volatiles released by $X$. perforans did not only attract the swarmer but also increased its dispersal without affecting its growth rate. Using fluorescent-stained X. perforans, Hagai et al. (2014) revealed that this hitch-hiking strategy also occurs on tomato leaves with different swarming bacterial species, suggesting that this might be a widespread and ecologically important phenomenon.

\section{Conclusions and perspectives}

Most studies to date have focused on the role of volatiles in plant-microbe interactions and their role in plant growth and health (Bitas et al., 2013, Penuelas 
et al., 2014). However, the role of volatiles in microbe-microbe communication and competition in soils remains largely unknown. It is not completely clear why microorganisms produce volatiles and what their exact functions are. It has been proposed that volatiles represent waste material or a detoxification system of the producing microorganisms (Claeson et al., 2007). However, from recent studies summarised in part 2 and 3 of this review, it is clear that microbial volatiles can have two major roles in a long-distance interactions in microbial communities as: (i) infochemical molecules affecting the behaviour, population dynamic and gene expression in the responding microorganism, and (ii) competitive tools directly exerting antimicrobial activity, providing an advantage by suppressing or eliminating potential enemies.

Currently, most studies on microbial volatiles are performed in vitro under nutrient rich conditions (Kai et al., 2009, Weise et al., 2012) and may not represent the conditions that prevail in the microbial environment. Furthermore, as indicated by (Garbeva et al., 2014, Garbeva et al., 2014), the composition of volatiles produced by a mixture of bacterial species can differ from those produced by each bacterial monoculture.

Soil is a complex, highly diverse and heterogeneous environment; an important characteristic of most soils is the occurrence of air-filled pores. Hence, the gaseous phase forms an important part of the natural surroundings of soil microorganisms. It has been estimated that the area of soil particles covered by microorganisms is less than $1 \%$, implying that the distance between microcolonies of microbial neighbours can be considerable (Young et al., 2008). Compared with diffusible compounds, volatile compounds can travel faster and over longer distances through both the liquid and gaseous phase of the soil (Insam \& Seewald, 2010, Effmert et al., 2012), which facilitate the interactions between soil microorganisms. Therefore, volatiles have an important role in the communication and competitiveness between physically separated soil microorganisms (Kai et al., 2009, Effmert et al., 2012, Garbeva et al., 2014). It is plausible that in soil, dormant microorganisms can sense changes in their environments via emitted volatiles and change their behaviour accordingly and in turn, influence the behaviour of other soil microorganisms (Garbeva et al., 2011). Although several studies have shown that volatile compounds can be used as signalling molecules in soil microbial communication, so far it is unclear how volatiles are perceived as signals by the 
microorganisms.

To date, little is still known about the regulatory pathways and genes involved in volatile biosynthesis, as well as the possible role of QS in the production of volatiles. Because the production of volatiles is often reported to vary depending on cell density (Weise et al., 2012, Groenhagen et al., 2013), it is tempting to reason that volatiles are regulated by QS. However, there are only few and contradictory reports regarding QS regulation of volatile production. Whereas for hydrogen cyanide in Pseudomonas and Chromobacterium species it was concluded to be QS regulated (Pessi \& Haas, 2000, Blom et al., 2011), for B. ambifaria, production of volatiles appeared not to be controlled by QS as the volatile profiles of the wild-type and the QS mutant were very similar (Groenhagen et al., 2013). Future challenges are therefore to further elucidate the large chemical diversity of microbial volatiles, to discover regulatory pathways and genes involved in the biosynthesis of volatiles in soil bacteria and fungi, to determine biologically relevant concentrations and to resolve the importance of volatiles in ecosystem processes. Monitoring volatiles may be used as a potential indicator of microbial activity, measuring shifts in community composition in the environment and ultimately for determining the soil health status of agricultural soils.

\section{Conflict of interest}

The authors declare no conflict of interest.

\section{Acknowledgements}

This work is supported by The Netherlands Organization for Scientific Research (NWO) VIDI personal grant to PG (864.11.015). This is publication 5808 of the Netherlands Institute of Ecology (NIOO-KNAW). We wish to thank the anonymous reviewers for their helpful comments. 
34 / Chapter 2 


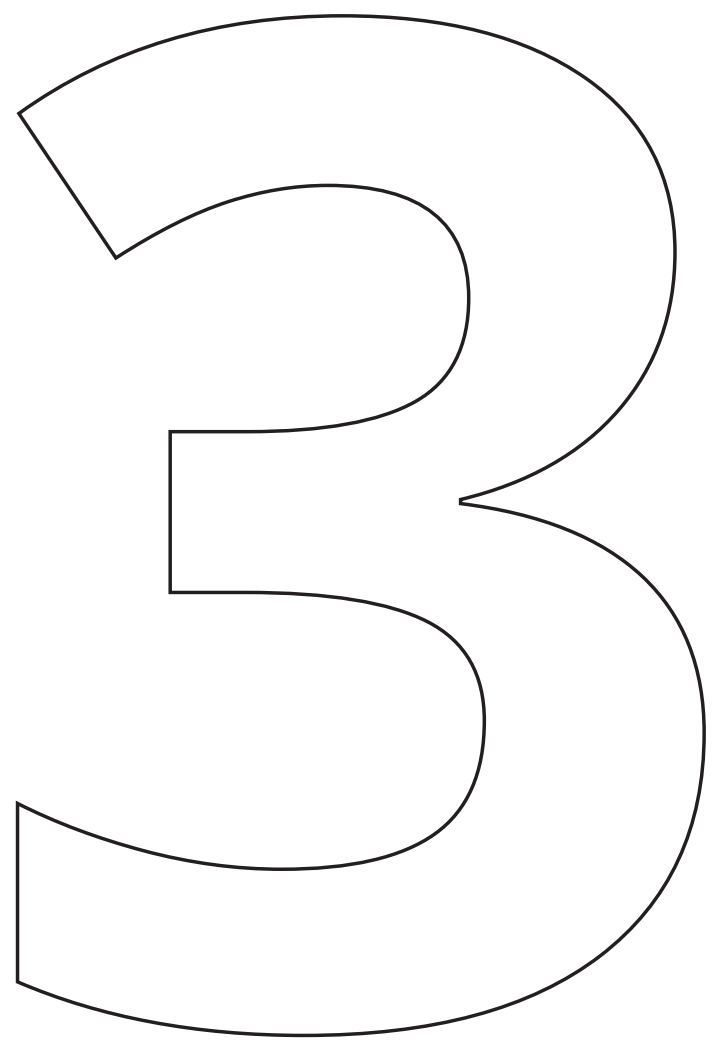

\section{Microbial small talk: Volatiles in fungal-bacterial interactions}

Ruth Schmidt, Desalegn W. Etalo, Victor de Jager, Saskia Gerards, Hans Zweers, Wietse de Boer, and Paolina Garbeva

Frontiers in Microbiology (2015) 



\section{Abstract}

There is increasing evidence that volatile organic compounds (VOCs) play an important role in the interactions between fungi and bacteria, two major groups of soil inhabiting microorganisms. Yet, most of the research has been focused on effects of bacterial volatiles on suppression of plant pathogenic fungi whereas little is known about the responses of bacteria to fungal volatiles. In the current study we performed a metabolomics analysis of volatiles emitted by several fungal and oomycetal soil strains under different nutrient conditions and growth stages. The metabolomics analysis of the tested fungal and oomycetal strains revealed different volatile profiles dependent on the age of the strains and nutrient conditions. Furthermore, we screened the phenotypic responses of soil bacterial strains to volatiles emitted by fungi. Two bacteria, Collimonas pratensis Ter291 and Serratia plymuthica PRI-2C, showed significant changes in their motility, in particular to volatiles emitted by Fusarium culmorum. This fungus produced a unique volatile blend, including several terpenes. Four of these terpenes were selected for further tests to investigate if they influence bacterial motility. Indeed, these terpenes induced or reduced swimming and swarming motility of S. plymuthica PRI-2C and swarming motility of $C$. pratensis Ter291, partly in a concentration-dependent manner. Overall the results of this work revealed that bacteria are able to sense and respond to fungal volatiles giving further evidence to the suggested importance of volatiles as signaling molecules in fungal-bacterial interactions.

Keywords: soil microorganisms, fungal-bacterial interactions, volatiles, terpenes, signaling, motility 


\section{Introduction}

In terrestrial ecosystems fungi and bacteria live in complex multi-species networks (Frey-Klett et al., 2011, Hung et al., 2015). Within those networks, both fungi and bacteria produce a plethora of secondary metabolites of diverse chemical classes (Schulz \& Dickschat, 2007, Morath et al., 2012, Muller et al., 2013). Several of these secondary metabolites are diffusible signaling molecules, such as antibiotics and antibiotic-like substances or quorum-sensing molecules, which are important in interactions between fungi and bacteria (Ryan \& Dow, 2008, Frey-Klett et al., 2011, Haq et al., 2014).

A group of metabolites that is increasingly recognized to play important roles in microbial interactions and communications are volatile organic compounds (VOCS). Those compounds are low molecular weight carbon-containing compounds (<400 Da) that evaporate easily at normal temperatures and air pressures (Schulz \& Dickschat, 2007, Bitas et al., 2013). Their physico-chemical properties facilitate evaporation and diffusion through both water- and gas-filled pores in soil and rhizosphere environments (Schmidt et al., 2015). Hence, they possess important functions for long distance fungal-bacterial interactions in the porous soil matrix.

Although it is known that many soil microorganisms produce a wide range of volatile compounds (Wheatley, 2002, Effmert et al., 2012) relatively little attention has been paid to fungal volatiles and to their ecological role. In these studies, over 300 distinct volatiles have been identified from fungi, belonging to different chemical classes including alcohols, benzenoids, aldehydes, alkenes, acids, esters and ketones (Morath et al., 2012, Lemfack et al., 2014). However, most research focused on volatiles produced by single species growing under nutrient rich conditions which is far from the nutrient-limited growth that most microbes experience in soil (Garbeva \& de Boer, 2009, Kai et al., 2009, Weise et al., 2012). Furthermore, it has been shown that the composition of volatiles can vary depending on several factors, such as the fungal growth stage, moisture, temperature and $\mathrm{pH}$ (Wheatley, 2002, Insam \& Seewald, 2010, Romoli et al., 2014).

Within the past years, it has become evident that microbial volatiles can play two major roles in long-distance interactions within soil microbial communities: (i) as infochemical molecules affecting the behavior, population dynamics and gene expression in responding microorganisms and (ii) as interference competition 
tools suppressing or eliminating potential enemies (Effmert et al., 2012, Garbeva et al., 2014, Schmidt et al., 2015). Currently, most research on microbial volatiles is focused on the effect of bacterial volatiles on other bacteria and/or fungi whereas the effect of fungal volatiles on bacteria remains largely unknown.

In this study, we aimed to profile volatiles emitted by a range of fungal and oomycetal soil strains and to test the effect of these volatiles on the behavior of phylogenetically different soil bacteria which are known from previous studies to interact with fungi (Leveau et al., 2010, Mela et al., 2011, Garbeva et al., 2014). The main research questions we addressed were (1) Can bacteria sense the presence of fungal and oomycetal volatiles and react with specific phenotypical responses and (2) Is the response dependent on the nutrient conditions and growth stage of the fungal and oomycetal strains?

\section{Material and Methods}

\section{Bacterial, fungal and oomycetal strains and growth conditions}

All bacterial, fungal and oomycetal strains used in this study (Table 1) have been isolated from bulk or rhizosphere soil. The bacterial strains Collimonas fungivorans Ter331 and Ter6, Collimonas pratensis Ter91, Ter291 and Collimonas arenae Ter10 and Ter282, Burkholderia sp. AD24, Pedobacter sp. V48 and Paenibacillus sp. AD87 are strains from sandy dune soils in The Netherlands (De Boer et al., 1998, de Boer, 2004). Serratia plymuthica PRI-2C strain was isolated from maize rhizosphere, The Netherlands (Garbeva et al., 2004). Bacterial strains were pre-cultured from frozen glycerol stocks on 0.1 Tryptic Soya Broth plates (0.1 TSB) $\left(5 \mathrm{~g} \mathrm{~L}^{-1} \mathrm{NaCl}, 1 \mathrm{~g} \mathrm{~L}^{-1} \mathrm{KH}_{2} \mathrm{PO}_{4^{\prime}}\right.$ $3 \mathrm{~g} \mathrm{~L}^{-1} \mathrm{TSB}, 20 \mathrm{~g} \mathrm{~L}^{-1}$ Merck Agar; $\mathrm{pH}=6.5$ ) (Garbeva \& de Boer, 2009) and grown for three days at $20^{\circ} \mathrm{C}$ prior usage.

The fungal strains Trichoderma harzianum PVDG2, Mucor hiemalis PVDG1 and Fusarium culmorum PV were also isolated from a sandy dune soil in The Netherlands (De Boer et al., 1998). Verticillium dahliae JR2 was isolated from tomato, Canada and Rhizoctonia solani AG2.2IIIB was isolated from sugar beet, the Netherlands (Garbeva et al., 2014). The fungal oomycete Pythium ultimum P17 was isolated from tulip bulb rhizosphere, The Netherlands (Garbeva et al., 2014). All fungal and oomycetal strains were pre-cultured on 0.5 Potato Dextrose Agar plates 
(19.5 g L'1 PDA, $7.5 \mathrm{~g} \mathrm{~L}^{-1}$ Merck Agar; pH=5.5-6) (Fiddaman \& Rossall, 1993) and incubated for 6 days at $20^{\circ} \mathrm{C}$ prior usage.

Table 1 / Bacterial, fungal and oomycetal strains used in this study

\begin{tabular}{|c|c|c|c|c|}
\hline & Phylum/Class & Source & $\begin{array}{l}\text { Accession } \\
\text { Number }\end{array}$ & Reference \\
\hline \multicolumn{5}{|l|}{ Bacterial strains } \\
\hline $\begin{array}{l}\text { Collimonas } \\
\text { fungivorans Ter331 }\end{array}$ & $\begin{array}{l}\text { Proteobacteria, } \\
\beta \text {-Proteobacteria }\end{array}$ & $\begin{array}{l}\text { Inner coastal dune } \\
\text { soil in Terschelling, } \\
\text { the Netherlands }\end{array}$ & NR_074756 & $\begin{array}{l}\text { (De Boer et al., } \\
\text { 1998, de Boer, } \\
\text { 2004) }\end{array}$ \\
\hline $\begin{array}{l}\text { Collimonas } \\
\text { fungivorans Ter6 }\end{array}$ & $\begin{array}{l}\text { Proteobacteria, } \\
\text { ß-Proteobacteria }\end{array}$ & $\begin{array}{l}\text { Inner coastal dune } \\
\text { soil in Terschelling, } \\
\text { the Netherlands }\end{array}$ & NR_028007 & $\begin{array}{l}\text { (De Boer et al., } \\
\text { 1998, de Boer, } \\
\text { 2004) }\end{array}$ \\
\hline $\begin{array}{l}\text { Collimonas pratensis } \\
\text { Ter91 }\end{array}$ & $\begin{array}{l}\text { Proteobacteria, } \\
\beta \text {-Proteobacteria }\end{array}$ & $\begin{array}{l}\text { Inner coastal dune } \\
\text { soil in Terschelling, } \\
\text { the Netherlands }\end{array}$ & - & $\begin{array}{l}\text { (De Boer et al., } \\
\text { 1998, de Boer, } \\
\text { 2004) }\end{array}$ \\
\hline $\begin{array}{l}\text { Collimonas pratensis } \\
\text { Ter291 }\end{array}$ & $\begin{array}{l}\text { Proteobacteria, } \\
\text { } \text {-Proteobacteria }\end{array}$ & $\begin{array}{l}\text { Inner coastal dune } \\
\text { soil in Terschelling, } \\
\text { the Netherlands }\end{array}$ & - & $\begin{array}{l}\text { (De Boer et al., } \\
\text { 1998, de Boer, } \\
\text { 2004) }\end{array}$ \\
\hline $\begin{array}{l}\text { Collimonas arenae } \\
\text { Ter10 }\end{array}$ & $\begin{array}{l}\text { Proteobacteria, } \\
\text { } \text {-Proteobacteria }\end{array}$ & $\begin{array}{l}\text { Inner coastal dune } \\
\text { soil in Terschelling, } \\
\text { the Netherlands }\end{array}$ & - & $\begin{array}{l}\text { (De Boer et al., } \\
\text { 1998, de Boer, } \\
\text { 2004) }\end{array}$ \\
\hline $\begin{array}{l}\text { Collimonas arenae } \\
\text { Ter282 }\end{array}$ & $\begin{array}{l}\text { Proteobacteria, } \\
\text { } \text {-Proteobacteria }\end{array}$ & $\begin{array}{l}\text { Inner coastal dune } \\
\text { soil in Terschelling, } \\
\text { the Netherlands }\end{array}$ & - & $\begin{array}{l}\text { (De Boer et al., } \\
\text { 1998, de Boer, } \\
\text { 2004) }\end{array}$ \\
\hline $\begin{array}{l}\text { Burkholderia sp. } \\
\text { AD24 }\end{array}$ & $\begin{array}{l}\text { Proteobacteria, } \\
\beta \text {-Proteobacteria }\end{array}$ & $\begin{array}{l}\text { Rhizosphere and } \\
\text { bulk soil of } C . \\
\text { arenaria }\end{array}$ & KJ685239 & $\begin{array}{l}\text { de Ridder- } \\
\text { Duine et al. } \\
\text { (2005) }\end{array}$ \\
\hline $\begin{array}{l}\text { Paenibacillus sp. } \\
\text { AD87 }\end{array}$ & Firmicutes, Bacilli & $\begin{array}{l}\text { Rhizosphere and } \\
\text { bulk soil of } C . \\
\text { arenaria }\end{array}$ & KJ685299 & $\begin{array}{l}\text { de Ridder- } \\
\text { Duine et al. } \\
\text { (2005) }\end{array}$ \\
\hline Pedobacter sp. V48 & $\begin{array}{l}\text { Bacteroidetes, } \\
\text { Sphingobacteriia }\end{array}$ & $\begin{array}{l}\text { Coastal dune soil, } \\
\text { the Netherlands }\end{array}$ & DQ778037 & $\begin{array}{l}\text { de Boer et al. } \\
(2007)\end{array}$ \\
\hline $\begin{array}{l}\text { Serratia plymuthica } \\
\text { PRI-2C }\end{array}$ & $\begin{array}{l}\text { Proteobacteria, } \\
\text { y-Proteobacteria }\end{array}$ & $\begin{array}{l}\text { Maize rhizosphere } \\
\text { soil, the } \\
\text { Netherlands }\end{array}$ & AJTB00000000 & $\begin{array}{l}\text { Garbeva et al. } \\
(2004)\end{array}$ \\
\hline
\end{tabular}

\section{Fungal and Oomycetal strains}

\begin{tabular}{|c|c|c|c|c|}
\hline $\begin{array}{l}\text { Trichoderma } \\
\text { harzianum PVDG2 }\end{array}$ & Ascomycota & $\begin{array}{l}\text { Coastal dune soil, } \\
\text { the Netherlands }\end{array}$ & KC888990 & $\begin{array}{l}\text { De Boer et al. } \\
\text { (1998) }\end{array}$ \\
\hline $\begin{array}{l}\text { Fusarium culmorum } \\
\text { PV }\end{array}$ & Ascomycota & $\begin{array}{l}\text { Coastal dune soil, } \\
\text { the Netherlands }\end{array}$ & - & $\begin{array}{l}\text { De Boer et al. } \\
\text { (1998) }\end{array}$ \\
\hline $\begin{array}{l}\text { Verticillium dahliae } \\
\text { JR2 }\end{array}$ & Ascomycota & Tomato soil, Canada & $\begin{array}{l}\text { CP009075.1,.., } \\
\text { СР009081.1 }\end{array}$ & Huang (2014) \\
\hline $\begin{array}{l}\text { Mucor hiemalis } \\
\text { PVDG1 }\end{array}$ & Zygomycota & $\begin{array}{l}\text { Coastal dune soil, } \\
\text { the Netherlands }\end{array}$ & KC888987 & $\begin{array}{l}\text { De Boer et al. } \\
\text { (1998) }\end{array}$ \\
\hline
\end{tabular}




\begin{tabular}{llll}
$\begin{array}{l}\text { Rhizoctonia solani } \\
\text { AG2.2IIIB }\end{array}$ & Basidiomycota & $\begin{array}{l}\text { Sugar beet } \\
\text { rhizosphere soil, the KT124637 } \\
\text { Netherlands } \\
\text { Pythium ultimum }\end{array}$ & $\begin{array}{l}\text { Rhizosphere } \\
\text { of bulb, the } \\
\text { P17 }\end{array}$ \\
\hline
\end{tabular}

\section{Screening for volatile-mediated phenotypes}

To investigate the effect of fungal volatiles on bacterial phenotypes, variations of assays in double plate-within-a-plate system (Lee et al., 2015) were performed (schematically described in Fig. S1). A 3.5-cm Petri dish containing the fungal and oomycetal strains was placed into a partitioned 9-cm Petri dish containing the bacterial strains. Plates containing only sterile medium was used as a control. The bacterial response to fungal and oomycetal volatiles was studied by comparing the phenotypic responses of the bacteria under the two nutrient conditions.

\section{Test of bacterial growth and antimicrobial activity}

The 3.5-cm Petri dish contained either $3 \mathrm{~mL} 0.5$ PDA medium or 1.5\% water-agar ( $5 \mathrm{~g} \mathrm{~L}^{-1} \mathrm{NaCl}, 1 \mathrm{~g} \mathrm{~L}^{-1} \mathrm{KH}_{2} \mathrm{PO}_{4^{\prime}} 15 \mathrm{~g} \mathrm{~L}^{-1}$ Merck Agar; $\mathrm{pH}$ 6.5) supplied with artificial root exudates (WA+ARE). The artificial root exudates stock solution contained 18.4 mM glucose; $18.4 \mathrm{mM}$ fructose; $9.2 \mathrm{mM}$ saccharose; $4.6 \mathrm{mM}$ citric acid; $9.2 \mathrm{mM}$ lactic acid; 6.9 mM succinic acid; $18.4 \mathrm{mM} \mathrm{L-serine;} 11 \mathrm{mM} \mathrm{L}$-glutamic acid and 18.4 $\mathrm{mM}$ L-alanine (C/N 10.4). Per liter of water-agar, $70.4 \mathrm{~mL}$ of ARE stock solution was added. A small plate $(3.5 \mathrm{~cm}$ ) containing the fungal and oomycetal plugs $(6 \mathrm{~mm}$ in diameter) was placed into the partitioned Petri dish $(9 \mathrm{~cm})$ and grown for 3 days at $20^{\circ} \mathrm{C}$ (Fig. S1 A). Bacterial strains were grown in $10 \mathrm{~mL} 0.1 \mathrm{TSB}$ broth overnight at 20 ${ }^{\circ} \mathrm{C}$. The cells were washed twice with sterile $10 \mathrm{~mm}$ sodium phosphate buffer (1.361 g K $\mathrm{KH}_{2} \mathrm{PO}_{4}$ in $1 \mathrm{~L}$ milliQ, pH 6.5), adjusted to a range of $1 \times 10^{6}-10^{2}$ cells $/ \mathrm{mL}, 5 \mu \mathrm{l}$ of cell suspension was spotted on 1.5\% WA+ARE of the partitioned Petri dish containing the fungal and oomycetal strains in the other compartment. The Petri dish was then closed and incubated for 3 days at $20^{\circ} \mathrm{C}$. On day 6, bacterial growth was determined by comparing the $\mathrm{cfu} / \mathrm{mL}$ of bacteria exposed to fungal and oomycetal volatiles to that of bacteria exposed only to sterile growth media only.

To test the triggering of antimicrobial activity by bacterial strains in response to fungal and oomycetal volatiles, an agar overlay assay was performed on day 6 (Tyc et al., 2014). The two indicator organisms E. coli WA321 and S. aureus 533R4 
were grown in liquid LB broth overnight at $37^{\circ} \mathrm{C}$, $220 \mathrm{rpm}$. Fresh LB-agar $(1.5 \%$ Merck Agar) was prepared, cooled down to $\sim 4{ }^{\circ} \mathrm{C}$ and the target organisms were added to $6 \times 10^{5}$ cells $/ \mathrm{mL}$ (E. coli WA321) or $4 \times 10^{5}$ cells $/ \mathrm{mL}$ (S. aureus 533R4). A volume of $5 \mathrm{~mL}$ liquid LB-agar containing the target organisms was poured over the compartment in which bacteria were growing. After solidification of the overlay agar, plates were incubated overnight at $37^{\circ} \mathrm{C}$. The next day, plates with bacteria exposed to fungal and oomycetal volatiles were examined for visible zones of inhibition (ZOI) compared to the bacteria exposed only to sterile media.

\section{Test of bacterial motility}

The effect of fungal and oomycetal volatiles on bacterial swarming and swimming motility was assessed on soft WA+ARE (0.6\% wt/vol and 0.3\% wt/vol, adapted from de Bruijn \& Raaijmakers (2009). After autoclaving, the medium was cooled down in a water bath to $60{ }^{\circ} \mathrm{C}$. Next, $10 \mathrm{~mL}$ of the medium was pipetted into the partitioned Petri dish and the plates were kept for $24 \mathrm{~h}$ at room temperature $\left(20^{\circ} \mathrm{C}\right)$ prior to the swarming and swimming assay. For all swarming and swimming assays, the same conditions (agar temperature \& volume, time period of storage of the poured plates) were kept constant to maximize reproducibility. A plate containing the fungal and oomycetal plugs (6 $\mathrm{mm}$ in diameter) that were grown on either 0.5 PDA or $1.5 \%$ water-agar supplied with artificial root exudates (WA+ARE) was placed into the partitioned Petri dish and grown for 3 days at $20^{\circ} \mathrm{C}$ (Fig. S1B). Recipient bacteria were grown in $10 \mathrm{~mL} 0.1 \mathrm{TSB}$ broth overnight at $20^{\circ} \mathrm{C}$. The cells were washed twice with sterile $10 \mathrm{~mm}$ sodium phosphate buffer (1.361 $\mathrm{g} \mathrm{KH}_{2} \mathrm{PO}_{4}$ in $1 \mathrm{~L}$ milliQ, pH 6.5), adjusted to $1 \times 10^{7}$ cells $/ \mathrm{mL}$ and $5 \mu$ of cell suspension was spotted in the center of the soft WA+ARE of the partitioned Petri dish containing the fungal and oomycetal strains. The Petri dish was then closed and incubated for 3 days at $20{ }^{\circ} \mathrm{C}$. On day 6 , volatile effect was determined by comparing the motility diameter of bacteria exposed to fungal and oomycetal volatiles to that exposed only to media. Motility diameter was calculated by measuring the radial swimming and swarming zones of the bacteria in two directions and calculating the mean for each of the three replicates.

\section{Test of bacterial biofilm formation}

The test for biofilm formation was adapted and modified from O'Toole et al. (1999). A small plate containing the fungal and oomycetal plugs ( $6 \mathrm{~mm}$ in diameter) that were 
grown on either 0.5 PDA or 1.5\% water-agar supplied with artificial root exudates (WA+ARE) was placed into the partitioned Petri dish and grown for 3 days at $20^{\circ} \mathrm{C}$ (Fig. S1C). Recipient bacteria were grown in $10 \mathrm{~mL} 0.1 \mathrm{TSB}$ broth overnight at $20^{\circ} \mathrm{C}$. The cells were washed twice with sterile $10 \mathrm{~mm}$ sodium phosphate buffer, adjusted to $1 \times 10^{7}$ cells $/ \mathrm{mL}$ and $20 \mu \mathrm{l}$ of cell suspension was added into 6-wells strip of a flatbottom 96-well plates made of transparent polystyrene (Greiner) with $180 \mu \mathrm{l} 0.1$ TSB broth per well. Part of the 96-well plates was placed into the partitioned Petri dish containing the fungal and oomycetal strains in the other compartment. The Petri dish was then closed and incubated for 2 days at $20^{\circ} \mathrm{C}$. On day 6 , the 6 -well strip was removed from the large Petri-dish and $10 \mu$ of $1 \%$ crystal violet solution was added to each well. These were incubated for 15 min at room temperature and rinsed three times with demi water. Biofilm formation was estimated by solubilization of crystal violet by adding $200 \mu \mathrm{l}$ of $96 \%$ ethanol and determining the OD600.Volatile activity was determined by comparing biofilm formation from bacteria exposed to fungal and oomycetal volatiles to that of bacteria exposed to media.

\section{Fungal and oomycetal volatile trapping and GC-Q-TOF analysis}

For the collection of fungal and oomycetal volatiles, glass Petri dishes with leads to which a steel trap containing 150 mg Tenax TA and 150 mg Carbopack B (Markes International Ltd., Llantrisant, UK) could be fixed were used (Garbeva et al., 2014). Fungi/oomyctes were grown on either 0.5 PDA or $1.5 \%$ water-agar supplied with artificial root exudates (WA+ARE) for 3 and 6 days at $20^{\circ} \mathrm{C}$. Petri dishes containing medium only served as controls. All treatments were inoculated in triplicates. The Tenax steel traps were collected at two time points for two fungal and oomycetal growth stages (day 3 and day 6) and under two nutrient conditions. Traps were removed, capped and stored at $4^{\circ} \mathrm{C}$ until analysis using GC-Q-TOF. Volatiles were desorbed from the traps using an automated thermodesorption unit (model UnityTD-100, Markes International Ltd., Llantrisant, UK) at $210{ }^{\circ} \mathrm{C}$ for $12 \mathrm{~min}(\mathrm{He}$ flow $50 \mathrm{~mL} / \mathrm{min}$ ) and trapped on cold trap at $-10{ }^{\circ} \mathrm{C}$. The trapped volatiles were introduced into the GC-QTOF (model Agilent 7890B GC and the Agilent 7200A QTOF, Santa Clara, USA) by heating the cold trap for 3 min to $280^{\circ} \mathrm{C}$ with split ratio set to $1: 20$. The column used was a $30 \times 0.25 \mathrm{~mm}$ ID RXI-5MS, film thickness 0.25 um (Restek 13424-6850, Bellefonte, PA, USA). Temperature program used was as follows: $39^{\circ} \mathrm{C}$ for $2 \mathrm{~min}$, from $39^{\circ} \mathrm{C}$ to $95^{\circ} \mathrm{C}$ at $3.5^{\circ} \mathrm{C} / \mathrm{min}$, then to $165^{\circ} \mathrm{C}$ at $6{ }^{\circ} \mathrm{C} /$ 
min, to $250{ }^{\circ} \mathrm{C}$ at $15{ }^{\circ} \mathrm{C} / \mathrm{min}$ and finally to $300{ }^{\circ} \mathrm{C}$ at $40{ }^{\circ} \mathrm{C} / \mathrm{min}$, hold $20 \mathrm{~min}$. The Volatiles were detected by the MS operating at $70 \mathrm{eV}$ in El mode. Mass spectra were acquired in full scan mode (30-400 amu, 4 scans/s). MassHunter Qualitative Analysis Software V B.06.00 Build 6.0.633.0 (Agilent Technologies, Santa Clara, USA) was used to control the instrument and for data acquisition and analysis. The mass chromatogram that were generated exported as mzData files and were processed (peak picking, baseline correction and peak alignment) in untargeted manner using the MetAlign software package (Lommen, 2009). Extraction and reconstitution of compound mass spectra were performed according to the method described previously by Tikunov et al. (2012). Identification of metabolites was performed using NIST-MS Search and accurate mass, retention indices and spectra match factor using NIST 2014 V2.20 (National Institute of Standards and Technology, USA, http:// www.nist.gov) and Wiley $9^{\text {th }}$ edition spectral libraries and by their linear retention indexes (Iri). The Iri values were compared with those found in the NIST and in the in-house NIOO Iri database.

\section{Test of terpene compounds on bacterial motility}

Four fungal volatiles, $\mathrm{a}$-Terpinene, $\beta$-Phellandrene, 3-Carene and Camphene were confirmed though injection of authentic standards obtained from Sigma Aldrich and the Natural Products Laboratory, Leiden. Volatile chemicals were dissolved in ethanol with concentrations of $10 \mathrm{nM}, 100 \mathrm{nM}, 10 \mu \mathrm{M}, 100 \mu \mathrm{M}, 10 \mathrm{mM}$ and $100 \mathrm{mM}$. The effect of individual terpene volatiles on the motility of $C$. pratensis Ter291 and S. plymuthica PRI-2C was investigated using the double plate-withina-plate system as described previously. The pure compounds were applied as a $10-\mu \mathrm{l}$ droplet on a sterile filter paper $(1 \times 1 \mathrm{~cm})$ on the bottom of a $3.5 \mathrm{~cm}$ Petri dish which was then transferred into the partitioned Petri dish. Plates were sealed immediately and incubated for 3 days at $20^{\circ} \mathrm{C}$. The activity of the pure compounds was determined by comparing the motility diameter of bacteria exposed to pure volatile compounds as single compounds to that of bacteria exposed to control (only ethanol). Motility diameter was calculated by measuring the radial swimming and swarming zones of the bacteria in two directions and calculating the mean for each of the three replicates. 


\section{Statistical analysis}

In all experiments, both for the treatments and the controls three independent replicates were considered. For the metabolomics analyses Genemaths XT software (Applied Maths, Belgium) was used for ANOVA (with Bonferroni correction), Principal Component Analysis (PCA) and Hierarchical Cluster Analysis. Pearson's correlation coefficients were used to calculate the distance or similarity between two entries and the resulting clusters were summarized using a complete linkage algorithm. The raw values of each sample were log-transformed and auto-scaled by the use of the average as an offset and the standard deviation as scale (raw value-average (offset)/SD (scale)).

Data obtained from the phenotypical assays were expressed as standard error of the mean and analyzed using OriginPro 2015 (OriginLab Corporation, MA) and SPSS (Science Inc., IL). Student's t test $(p<0.05)$ and one-way analysis of variance (ANOVA) between groups (treatments and control) were performed for all data.

\section{Results}

\section{Volatile profiles of fungal and oomycetal strains}

Based on metabolomics analysis a total of 306 putative volatiles were detected when the fungal and oomycetal strains were grown on the nutrient poor WA+ARE medium. 106 of these volatiles differed significantly in abundance between at least two of the fungal/oomycetal strains. A total of 578 putative volatiles were detected from the head space of the fungal and oomycetal strains when grown on the nutrient rich PDA with 173 volatiles significantly different in their abundance between at least two fungal/oomycetal strains. Volatiles that differed significantly were further used to compute Principal Component Analysis (PCA) and Hierarchical Cluster Analysis (HCA). In the PCA, the first three principal components (PC) explained 63\% of the total variation observed between the fungal/oomycetal strains that were grown on nutrient poor medium (Fig. 1). The first principal component PC1 explained $29 \%$ of the total variation and is primarily attributed to volatiles that were altered depending on the growth stage of the fungal/oomycetal strains (Fig. 1). The emission profile of these volatiles is indicated in cluster 5 of the HCA and is characterized by 
volatiles with higher abundance at the early stage of growth in all strains (Fig. S2A). Volatiles in cluster 4 of the HCA also showed higher emission in four out of the six strains (namely M. hiemalis, R. solani, T. harzianum, F. culmorum) at the early stage of growth while their emission pattern and abundance was fairly similar for the remaining two strains ( $P$. ultimum and $V$. dahliae) at both growth stages. The second PC explained $21 \%$ of the total variation and is attributed to the volatiles indicated in cluster 6 and 7 of the HCA (Fig. 1 and Fig. S2A, cluster 6 and 7). Volatiles in cluster 6 are primarily consisting of terpenes and were largely produced by F. culmorum at both growth stages with increased emission at the later growth stage (Fig. S2A). Volatiles in cluster 7 were abundantly detected in F. culmorum and T. harzianum at both growth stages with a noticeable increase in emission at the later growth stage. Some volatiles within this cluster were also detected in $V$. dahliae and were increasingly abundant at the later growth stage (Fig. S2A, cluster 7). The third PC explained $13 \%$ of the total variation. The volatiles that explained this variation are indicated in cluster 2 of the HCA and are mainly emitted by P. ultimum at the later stage of growth (Fig. S2A, cluster 2). 


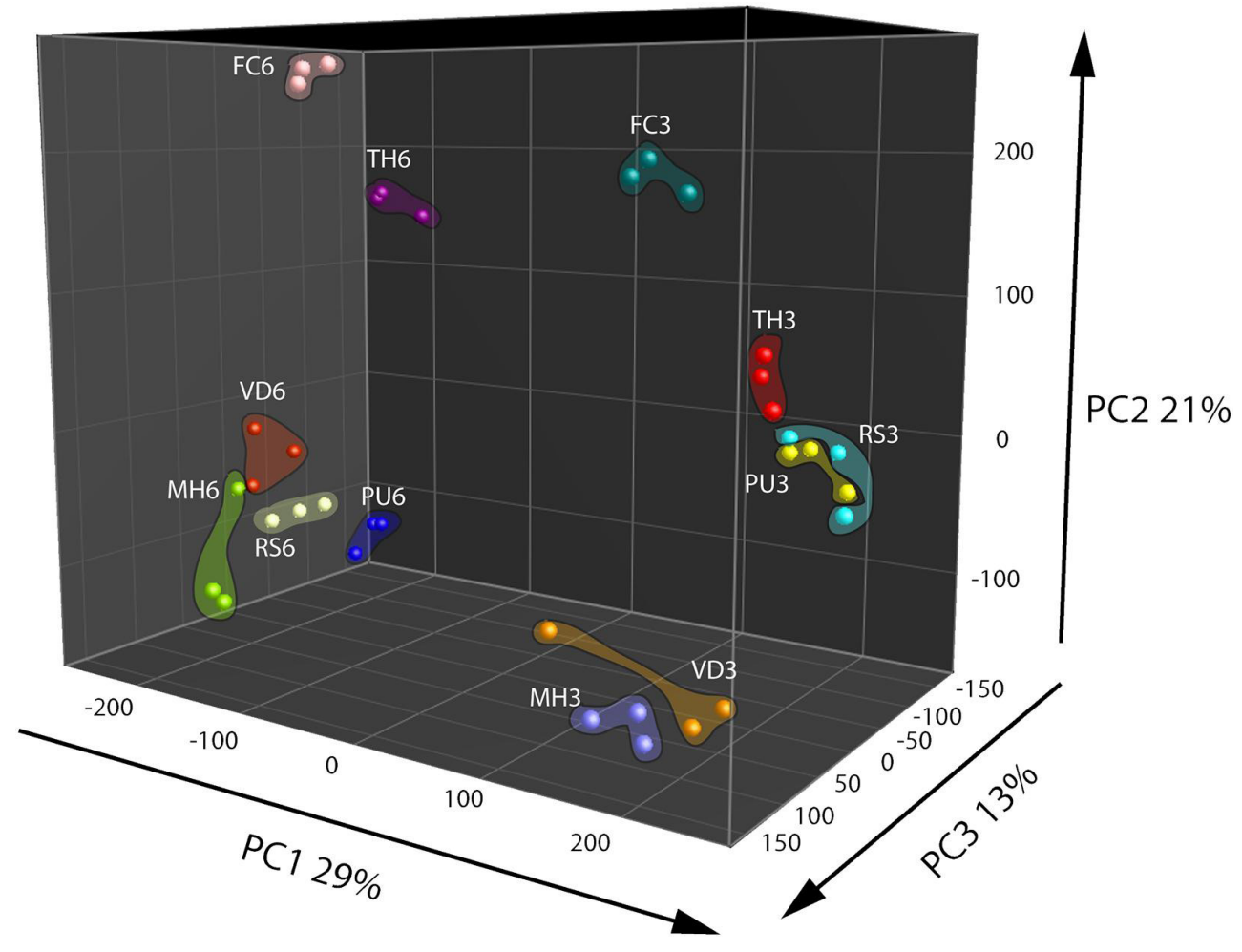

Fig. 1 / Principal Component Analysis (PCA) of fungal and oomycetal strains based on 106 volatiles that were significantly different $(P<0.05$ with Bonferroni correction) in abundance between at least two strains when grown on water agar supplied with artificial root exudates at day 3 (early growth stage) and day 6 (late growth stage). MH, Mucor hiemalis; RS, Rhizoctonia solani; PU, Pythium ultimum; VD, Verticillium dahliae; FC, Fusarium culmorum; TH, Trichoderma harzianum.

PCA based on volatiles from the fungi and oomycete grown on nutrient rich PDA showed that the first three PCs explained $60 \%$ of the total observed variation between the strains (Fig. 2). The first PC that explained 37\% of the total variation is related to compounds that are found in cluster 3 of the HCA (Fig. 2 and Fig. S2B, cluster 3). This cluster is characterized by higher emission of the volatiles at the early growth stage by all strains except P. ultimum and V. dahlia. The second PC explained $14 \%$ of the variation and is primarily associated to volatiles grouped in the cluster 6 of the HCA that predominantly explains the growth stage dependent emission of volatiles by the strains (Fig. 2 and Fig. S2B, cluster 6). Similar to the observation under the nutrient poor conditions, this group of volatiles showed higher emission at the early growth stage in all the strains. The third PC explained $8 \%$ of the total 
variation and the volatiles associated to this variation are indicated in cluster 2 of the HCA (Fig. 2 and Fig. S2B, cluster 2). This cluster consisted of terpenes and they are emitted predominantly by F. culmorum at the later growth stage. Although these terpenes and other volatiles in this cluster were emitted by F. culmorum on both media, they were emitted to higher extent at the early stages of growth on WA+ARE (Fig. S2A, cluster 6 and Fig. S2B, cluster 2).

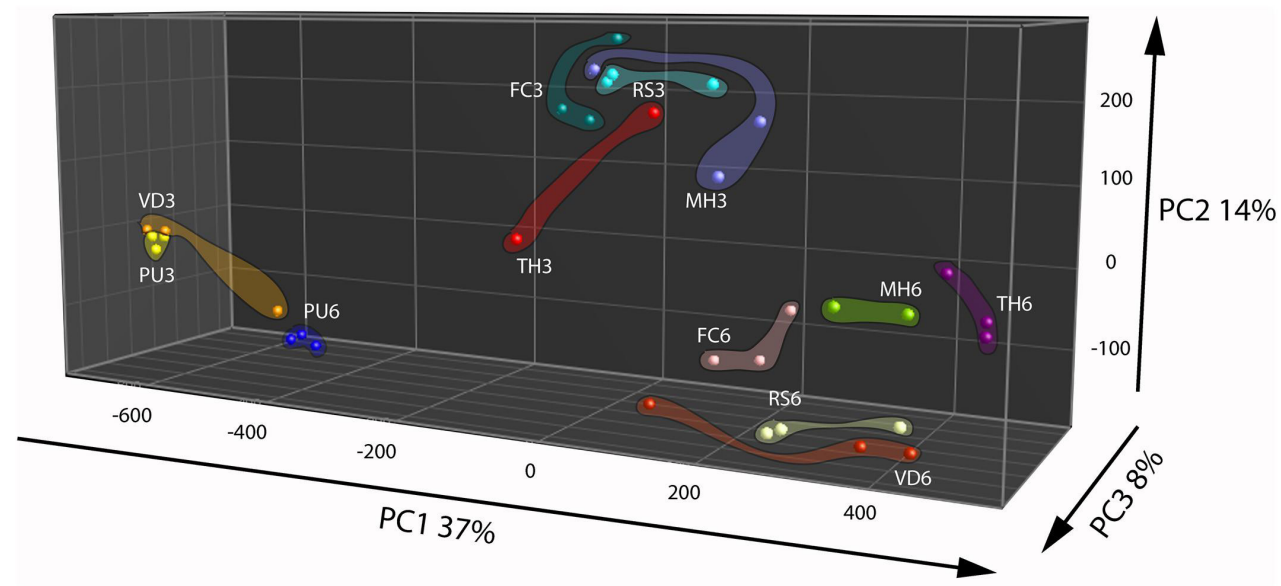

Fig. $\mathbf{2}$ / Principal Component Analysis of fungal and oomycetal strains based on 173 volatiles that were significantly different $(P<0.05$ with Bonferroni correction) in their abundance at least between two strains when grown on potato dextrose agar at day 3 (early growth stage) and day 6 (late growth stage). $\mathrm{MH}$, Mucor hiemalis; RS, Rhizoctonia solani; PU, Pythium ultimum; VD, Verticillium dahliae; FC, Fusarium culmorum; TH, Trichoderma harzianum.

The volatiles belonging to the unique clusters of $F$. culmorum were investigated in more detail for both nutrient poor and nutrient rich conditions. Cluster 6 consisted of 19 volatiles belonging to the classes of terpenes (monoterpenes and sesquiterpenes), alkylbenzenes, cycloalkenes and furans and Cluster 2 consisted of 17 volatiles belonging to the classes of terpenes (monoterpenes and sesquiterpenes), alkaloids, benzenoids and furans (Table 2). Some volatiles could not be identified and are thus indicated as unknown compounds with their respective retention times and accurate masses. In both clusters terpenes represented the most abundant class with unique volatiles for both nutrient conditions. The identity of the terpenes a-Terpinene, $\beta$-Phellandrene, 3-Carene and Camphene were confirmed with commercially available authentic standards. 
Table 2 / Characteristics of volatile compounds of cluster 6 (water agar supplied with artificial root exudates) and 2 (potato dextrose agar) emitted by F. culmorum.

\begin{tabular}{|c|c|c|c|c|c|c|}
\hline$\#$ & Compound & MSI* & $\begin{array}{l}\text { Average } \\
\mathrm{RT}^{\star \star}(\mathrm{min})\end{array}$ & $\begin{array}{l}\text { Accurate } \\
\text { mass }\end{array}$ & $\mathrm{RI} * * *$ & Class \\
\hline & & \multicolumn{5}{|c|}{ Cluster 6 WA+ARE } \\
\hline 1 & 2-Furancarboxaldehyde & 2 & 4.73 & & 771 & Furans \\
\hline 2 & Unknown & & 12.41 & 77.038 & & \\
\hline 3 & a-Phellandrene & 2 & 13.61 & & 1005 & Monoterpenes \\
\hline 4 & Pentamethylcyclopentadiene & 2 & 13.88 & & 1006 & \\
\hline 5 & 3-Carene & 1 & 14.33 & & 1017 & Monoterpenes \\
\hline 6 & o-Cymene & 2 & 14.42 & & 1026 & Alkylbenzenes \\
\hline 7 & Unknown & 2 & 21.48 & 93.067 & 1197 & \\
\hline 8 & Unknown & & 27.60 & 93.068 & & \\
\hline 9 & Unknown & & 29.22 & 229.001 & & \\
\hline 10 & a-Copaene & 2 & 29.52 & & 1433 & Sequiterpenes \\
\hline 11 & $\begin{array}{l}\text { 1,3-Cyclopentadiene-1- } \\
\text { butanenitrile, a-ethyl- }\end{array}$ & 2 & 29.73 & & 1433 & Cycloalkenes \\
\hline 12 & Unknown & & 29.84 & 161.128 & & \\
\hline 13 & (-)-Isoledene & 2 & 30.38 & & 1472 & Sequiterpenes \\
\hline 14 & Unknown & & 30.51 & 93.067 & & \\
\hline 15 & Unknown & & 31.09 & 67.053 & & \\
\hline 16 & Unknown & & 31.83 & 80.059 & & \\
\hline \multirow[t]{2}{*}{17} & cis-Farnesol & 2 & 32.59 & & 1503 & Sequiterpenes \\
\hline & & Cluste & 2 PDA & & & \\
\hline 1 & 2,4-Dimethylfuran & 2 & 4.08 & & 714 & Furans \\
\hline 2 & Unknown & & 4.88 & 95.047 & & \\
\hline 3 & Camphene & 1 & 12.79 & & 970 & Monoterpenes \\
\hline 4 & a-Terpinene & 1 & 13.85 & & 1004 & Monoterpenes \\
\hline 5 & $\beta$-Phellandrene & 1 & 14.55 & & 1032 & Monoterpenes \\
\hline 6 & 1,3,8-p-Menthatriene & 2 & 19.03 & & 1136 & Sesquiterpenes \\
\hline 7 & 2,6-Dichloroanisol & 2 & 20.65 & & 1157 & Benzenoids \\
\hline 8 & Unknown & & 25.42 & 189.164 & & \\
\hline 9 & Unknown & & 27.76 & 121.097 & & \\
\hline 10 & Longifolene & 2 & 27.91 & & 1347 & Sesquiterpenes \\
\hline 11 & Ledene & 2 & 27.98 & & 1348 & Sesquiterpenes \\
\hline 12 & y-Muurolene & 2 & 28.28 & & 1356 & Sesquiterpenes \\
\hline 13 & Streptazone C & 2 & 29.47 & & 1411 & Alkaloids \\
\hline
\end{tabular}




\begin{tabular}{|c|c|c|c|c|c|c|}
\hline 14 & Germacrene-D & 2 & 29.77 & & 1433 & Sesquiterpenes \\
\hline 15 & §-Guaiene & 2 & 30.04 & & 1412 & Sesquiterpenes \\
\hline 16 & Unknown & & 30.31 & 105.068 & & \\
\hline 17 & Unknown & & 30.67 & 67.054 & & \\
\hline 18 & Unknown & 2 & 31.77 & 109.100 & 1471 & \\
\hline 19 & a-Bisabolene & 2 & 32.55 & & 1500 & Sesquiterpenes \\
\hline
\end{tabular}

*1: Identified metabolites based on authentic standards; 2: Putatively annotated compounds (e.g. without chemicals reference standards, based upon physicochemical properties and/or spectral similarity with public/commercial NIST 2014 V2.20 and Wiley 9th edition spectral libraries). The reporting grades ( 1 and 2 ) are assigned according to the proposed minimum reporting standards for chemical analysis (metabolomics standards initiative (MSI)) (Sumner et al., 2007).

$* *$ Retention time
$* * *$ Retention index 


\section{Screening for bacterial phenotypes in response to fungal and oomycetal volatiles}

A screening using variations of assays of a double-plate-within-a-plate system was performed to test phenotypical responses of 10 bacterial strains to volatiles emitted by 6 fungal and oomycetal strains. The two different media, WA+ARE and PDA, for which fungal and oomycetal volatile production was analyzed, were also used for screening the bacterial response to volatiles. Out of all phenotypes tested (growth, antimicrobial activity, biofilm formation and motility), motility was the only phenotype affected by the fungal volatiles.

From all bacterial strains screened, C. pratensis Ter291 and S. plymuthica PRI-2C revealed the strongest and highly reproducible responses in motility upon exposure to the fungal and oomycetal volatiles (Fig. 3, Table S1). For the other bacterial strains high variability was observed between replicates (Table S1). Consequently, we focused on the description of the response in motility of $C$. pratensis Ter291 and S. plymuthica PRI-2C.
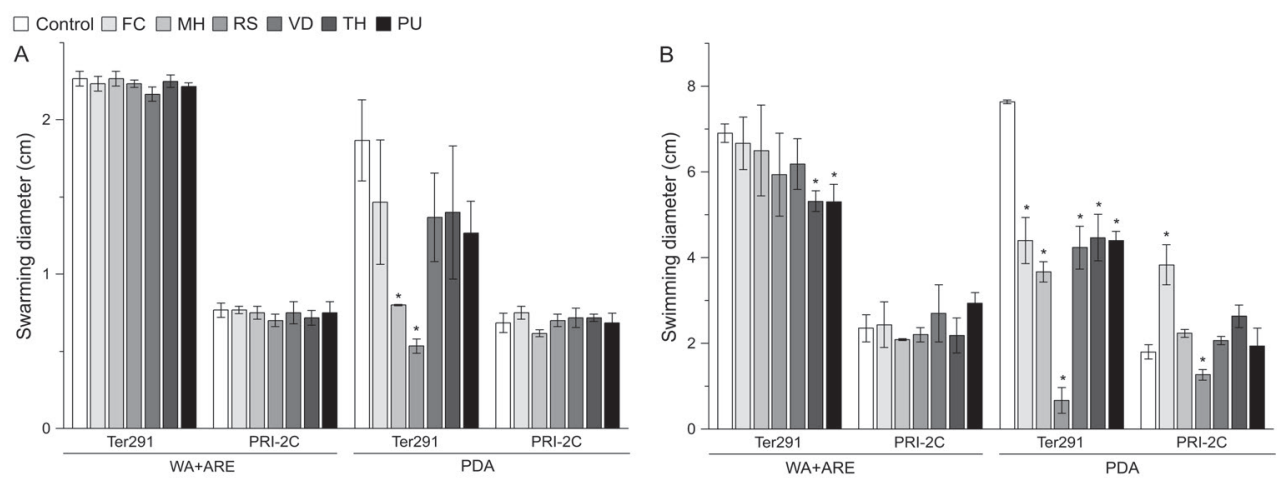

C

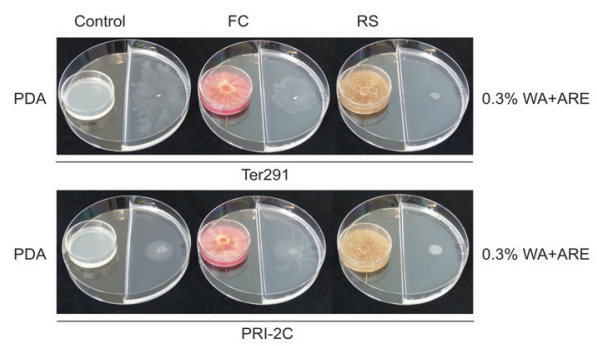

Fig. 3 / Effect of fungal and oomycetal volatiles on Collimonas pratensis Ter291 and Serratia plymuthica PRI$2 \mathrm{C}$ swarming motility ( $0.6 \%$ wt/vol agar) and swimming motility $(0.3 \%$ wt/vol agar) on water agar supplied 
with artificial root exudates (A) and potato dextrose agar (B). Setup of experiment and effect of FC and RS volatiles on swimming motility of C. pratensis Ter291 and S. plymuthica PRI-2C (C). MH, M. hiemalis; RS, R. solani; PU, P. ultimum; VD, V. dahliae; FC, F. culmorum; TH, T. harzianum, Control: media. Five microliters of washed overnight cultures of C. pratensis Ter291 and S. plymuthica PRI-2C was spotted in the center of a soft agar partitioned Petri dish containing the fungal and oomycetal strains and incubated for 3 days at $20^{\circ} \mathrm{C}$. As an indicator of motility the average swimming and swarming diameter $(\mathrm{cm})$ was measured. The error bars represent standard errors of the mean of three independent biological replicates. The asterisks indicate statistically significant $(P<0.05)$ differences relative to the control.

Overall, swimming motility was more strongly affected than swarming motility and the effect on motility was much more pronounced by fungal and oomycetal volatiles emitted from PDA than from WA+ARE (Fig. 3). Only C. pratensis Ter291 swarming motility was significant reduced when exposed to volatiles emitted by $M$. hiemalis and $R$. solani on PDA (Fig. 3A). No significant effect was observed in swarming motility of C. pratensis Ter291 and S. plymuthica PRI-2C by fungal and oomycetal volatiles emitted from WA+ARE and for S. plymuthica PRI-2C by volatiles emitted from PDA (Fig. 3A).

The swimming motility of C. pratensis Ter291 was significantly reduced by volatiles emitted by T. harzianum and P. ultimum growing on WA+ARE (Fig. 3B). No such effect was observed for S. plymuthica PRI-2C.Volatiles emitted by all fungal and oomycetal strains growing on PDA revealed a significant reduction of the swimming motility of C. pratensis Ter291 with a very strong effect observed by R. solani (Fig. 3B and C). For S. plymuthica PRI-2C swimming motility was significantly induced when exposed to volatiles produced by F. culmorum on PDA whereas the swimming motility was significantly reduced when exposed to volatiles produced by $R$. solani on PDA (Fig. 3B and C).

Volatiles emitted by $R$. solani growing on PDA reduced swarming as well swimming motility (Fig. 3B and C). The hierarchical cluster analysis (Fig. 2B) resulted in a unique cluster for $R$. solani, however the compounds could not be identified with certainty and thus no correlation between the reduction in motility and the potentially involved compounds could be drawn.

Interestingly, volatiles emitted by one fungus lead to different responses in the two strains. For example F. culmorum grown on PDA revealed a reduction of swimming motility of C. pratensis Ter291 and an induction in S. plymuthica PRI2C (Fig. 3B and C). As shown in the hierarchical cluster analysis, F. culmorum is characterized by a unique cluster of volatiles, consisting primarily of terpenes (Fig. 1B). 


\section{Effect of individual terpenes on S. plymuthica PRI-2C and C. pratensis Ter291 motility}

To test whether terpenes may play a role in the observed motility response of S. plymuthica PRI-2C and C. pratensis Ter291, four terpenes (a-Terpinene, $\beta$-Phellandrene, 3-Carene and Camphene) were selected from the unique $F$. culmorum cluster to be tested individually. These compounds showed a reliable annotation based on their retention indices and mass spectral similarity, with a match score $>800$, and which were commercially (synthetically) available as authentic standards. The identity of the four pure compounds was confirmed by GC-MS by comparing their mass spectra and RI with those found in the F. culmorum volatile profile. Their respective mass spectra are given in Fig. S3. A range of different concentrations, previously reported in experiments with microorganisms (Blom et al., 2011, Kim et al., 2013), was used to test their effect on motility of S. plymuthica PRI-2C and C. pratensis Ter291.

The screening showed that S. plymuthica PRI-2C was affected in both swarming and swimming motility, while C. pratensis Ter291 was only affected in swarming motility (Fig. 4). For some of the pure compounds, a concentration dependent effect was observed. For example, a -Terpinene affected S. plymuthica PRI-2C swimming motility in concentrations of $10 \mathrm{~nm}, 100 \mathrm{nM}$ and $100 \mu \mathrm{M}$, but no effect was observed with high concentrations (10 mM and $100 \mathrm{mM}$ ) (Fig. 4D). B-Phellandrene induced swimming motility in S. plymuthica PRI-2C in concentrations from $10 \mu \mathrm{M}$ to $100 \mathrm{mM}$ (Fig. 4D). Interestingly, depending on the concentration, 3-Carene affected the swarming motility in C. pratensis Ter291 in different ways. At concentrations of $10 \mathrm{nM}$ and $100 \mathrm{nM}$, C. pratensis Ter291 swarming motility was significantly increased while being significantly decreased at concentration of $10 \mu \mathrm{M}$ (Fig. 4A). Independently of the concentrations applied, ß-Phellandrene significantly reduced swarming motility of C. pratensis Ter291 (Fig. 4A). Likewise, 3-Carene and Camphene lead to a significant decrease in S. plymuthica PRI-2C swarming and swimming motility, respectively (Fig. 4B and D). 

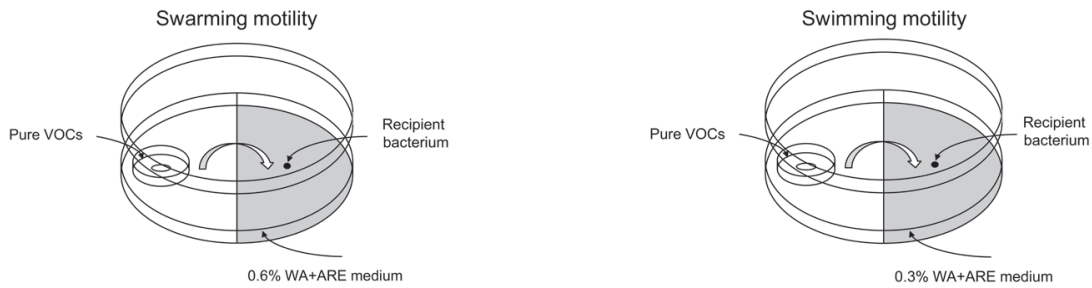

$\square$ Control $\square 10 \mathrm{nM} \square 100 \mathrm{nM} \square 10 \mu \mathrm{M} \square 100 \mu \mathrm{M} \square 10 \mathrm{mM}$
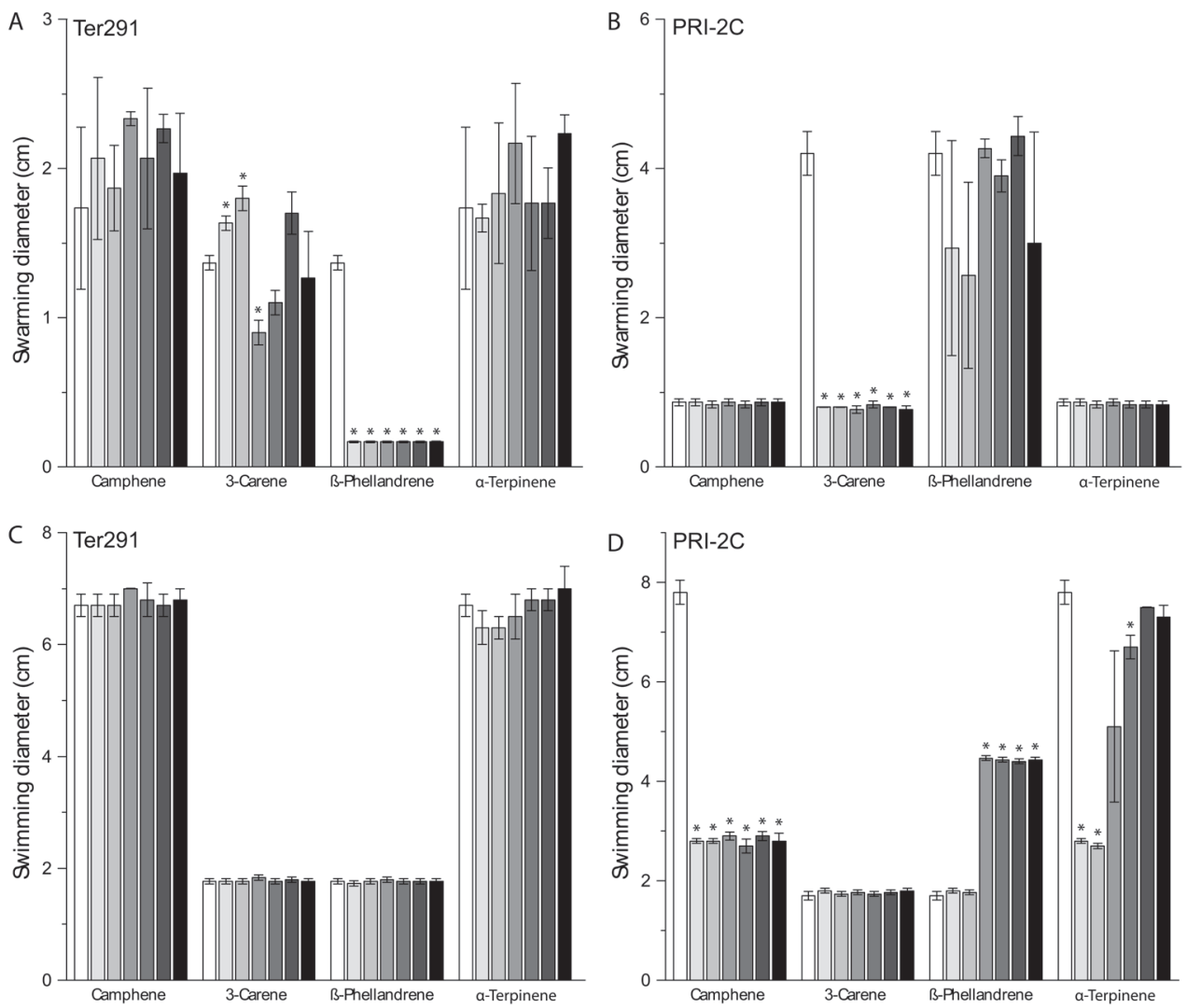

Fig. 4 / Setup of experiment and effect of pure volatiles on Collimonas pratensis Ter291 and Serratia plymuthica PRI-2C swarming motility (0.6\% wt/vol agar) (A,B) and swimming motility (0.3\% wt/vol agar) $(\mathbf{C}, \mathbf{D})$ on water agar supplied with artificial root exudates. Control: media. Five microliters of washed overnight cultures of C. pratensis Ter291 and S. plymuthica PRI-2C was spotted in the center of a soft agar partitioned Petri dish containing the pure volatiles and incubated for 3 days at $20^{\circ} \mathrm{C}$. As an indicator of motility the average swimming and swarming diameter $(\mathrm{cm})$ was measured. The error bars represent standard errors of the mean of three independent biological replicates. The asterisks indicate statistically significant $(P<0.05)$ differences relative to the control. 


\section{Discussion}

Volatile compounds form an important part in the interactions between different soil inhabiting microorganisms (Insam \& Seewald, 2010, Wenke et al., 2010, Effmert et al., 2012, Garbeva et al., 2014). They can have different ecological functions, including inhibition or promotion of other (micro)-organisms (Kai et al., 2007, Vespermann et al., 2007, Kai et al., 2009, Bailly \& Weisskopf, 2012, Bitas et al., 2013). However, one important role that has been long overlooked is the ability of volatiles to act as signaling molecules in the communication between different soil microorganisms despite their physico-chemical properties that facilitate diffusion through soil. To date, very little is known about the role of fungal volatiles in fungalbacterial interactions. Thus, the aim of this study was to investigate the effect of fungal volatiles on bacteria.

Our results added to this gap in knowledge by showing that fungal and oomycetal volatiles can play an important role in long distance fungal-bacterial interactions, and can lead to specific phenotypical responses in the interacting partners. Out of all the phenotypical responses considered namely growth, antimicrobial activity, biofilm formation and motility, motility of bacteria, both swimming (individual cells moving in more liquid environments) and swarming (direct, signal-dependent movement powered by rotating flagella), were significantly affected. Fungal and oomycetal volatiles either triggered or suppressed bacterial motility depending on the interacting partner. This finding could, therefore, reflect a potential strategy employed by the fungus to attract mutualistic bacteria towards itself and to repel competitors from common niches by manipulating their motility through volatiles. The composition and abundance of volatiles is affected by the growth stage of the fungal/oomycetal strains and the nutrient conditions. Several independent studies have reported that the volatile profiles of bacteria are also dependent on growth condition and nutrient availability (Korpi et al., 2009, Insam \& Seewald, 2010, Blom et al., 2011, Bitas et al., 2013, Garbeva et al., 2014).

Besides the growth stage- and nutrient condition-dependent changes in the global volatile profile, certain groups of volatiles are emitted in higher amounts by specific individual strains. Terpenes emitted by F. culmorum are the most salient example from our study: a nutrient-poor growing condition triggers higher levels of terpene emission at an early growth stage and an even higher emission at a later growth stage; on the contrary, under nutrient-rich conditions, the emission of this 
volatile cluster was induced only at a later growth stage. This suggests that fungi and oomycetes can invest their carbon resources towards formation of specific blends of volatiles depending on their growth stages and the nutrient availability in their environment. Our findings are in line with those by Korpi et al. (2009), who demonstrated that a lack of certain nutrients leads to terpene emission, suggesting that some volatiles are produced only under nutrient-limited conditions, which is often the case in natural environments. Terpenes represent the biggest and most diverse family of primary and secondary metabolites found in a variety of organisms, among which several fungi (Keller et al., 2005, Gioacchini et al., 2008, Strobel et al., 2011, Muller et al., 2013, Busko et al., 2014). Most studies, however, focused mainly on the detection and chemical characterization of these molecules, while only few addressed the biological function of terpenes. The latter studies indicate that fungal terpenes may be used in defense against competitors (e.g. caryophyllene) or as a signaling molecule (e.g. farnesol) (Martins et al., 2007). Our work, based on the screening of fungal and oomycetal strains and using pure terpene compounds, proved that individual terpenes affect the motility of the exposed bacteria.

Several studies showed that fungi have a high sensitivity to volatiles emitted by bacteria leading to reduction and inhibition in spore germination and growth (fungistasis) (Garbeva et al., 2011, Effmert et al., 2012, Garbeva et al., 2014, Schmidt et al., 2015). The difference in susceptibility between fungi and oomycetes may be due to the structure of their cell wall (Schmidt et al., 2015). It was recently proposed that bacteriostasis (inability of bacteria to multiply in soil) (Ho \& Ko, 1982, Effmert et al., 2012) might also involve volatile compounds. However, within this study we did not observed effect on bacterial growth by fungal volatiles. In contrast to fungi, bacteria seem to be more resistant to volatiles. It has been speculated that variations in sensitivity of bacteria to volatiles may possibly be mediated by an ATPdependent efflux mechanism, which has been investigated for several terpene compounds against $P$. aeruginosa (Cox \& Markham, 2007) as well as the ability of volatiles to disintegrate the outer membrane (Longbottom et al., 2004). These findings may indicate that bacteria are more resistant to volatiles emitted by fungi and oomycetes.

The identity of volatile molecules is an important basis for understanding their ecological roles. However, it is a challenging task to unambiguously identify the high number of compounds detected, just as it is to set the right ranges of 
concentrations that are representative of the natural condition during screenings with pure compounds. The technology used in this study does not allow measuring the actual concentration of the volatile compounds produced by the strains. Thus, when testing the effect of pure compounds we adopted a range of concentrations known to be relevant in such assays (Blom et al., 2011, Kim et al., 2013). Interestingly, some of the pure compounds showed a dose-dependent effect on the motility. This suggests that by regulating the emission of volatiles, fungi might be able to influence bacterial responses in different ways. For instance, the emission of volatiles in lower concentrations might attract the bacterium to move towards the fungus, while volatiles emitted in higher concentrations might be toxic and thus repel the bacteria away from the fungus. For example, bacteria from the genus Collimonas, used in this study, were previously shown to colonize and grow on living fungal hyphae (a phenomenon called mycophagy) (de Boer, 2004), implying that volatiles might play a role as long-distance signals for attracting such mycophagous bacteria.

\section{Conclusion}

Bacteria can sense fungal and oomycetal volatiles and respond with changes in motility. This response was dependent on the volatile blend emitted by the organisms, which was influenced by the nutrient conditions and, for some strains, by their growth stage. Several identified volatile terpenes were shown to affect motility. To better understand how bacteria perceive fungal volatiles on a cellular level, a valuable insight could stem from future studies involving transcriptomics and proteomics tools.

\section{Conflict of Interest Statement}

The authors declare that the research was conducted in the absence of any commercial or financial relationships that could be construed as a potential conflict of interest.

\section{Acknowledgments}

We thank Sacha Hilversum and Lennart Backus for their help in bioinformatics analysis using RFScout and Prof. Gilles van Wezel for providing us with $\beta$-Phellandrene for this study. Paolina Garbeva is financed by The Netherlands Organization for Scientific Research (NWO) VIDI personal grant (864.11.015). This is publication 5980 of the NIOO-KNAW. 


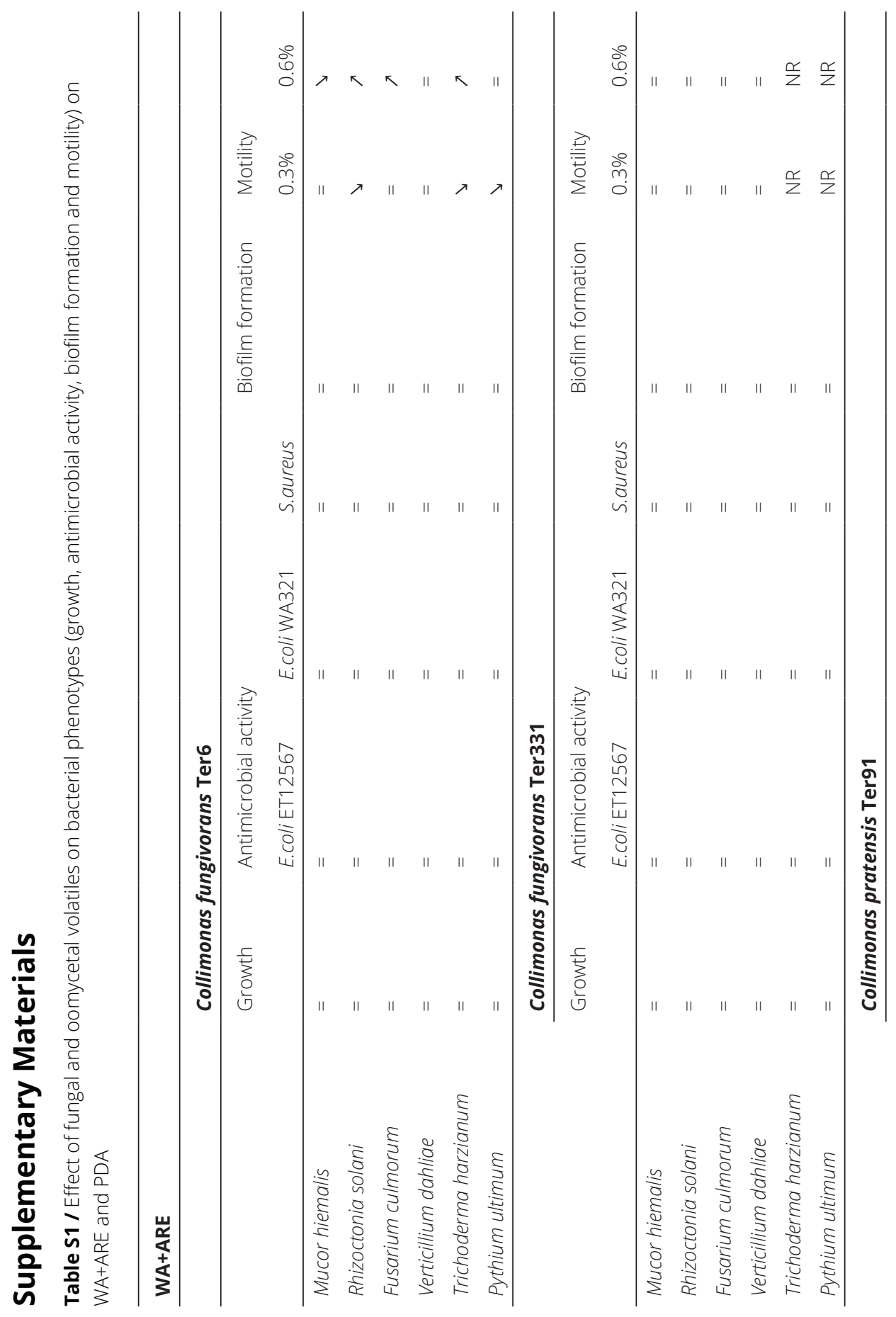




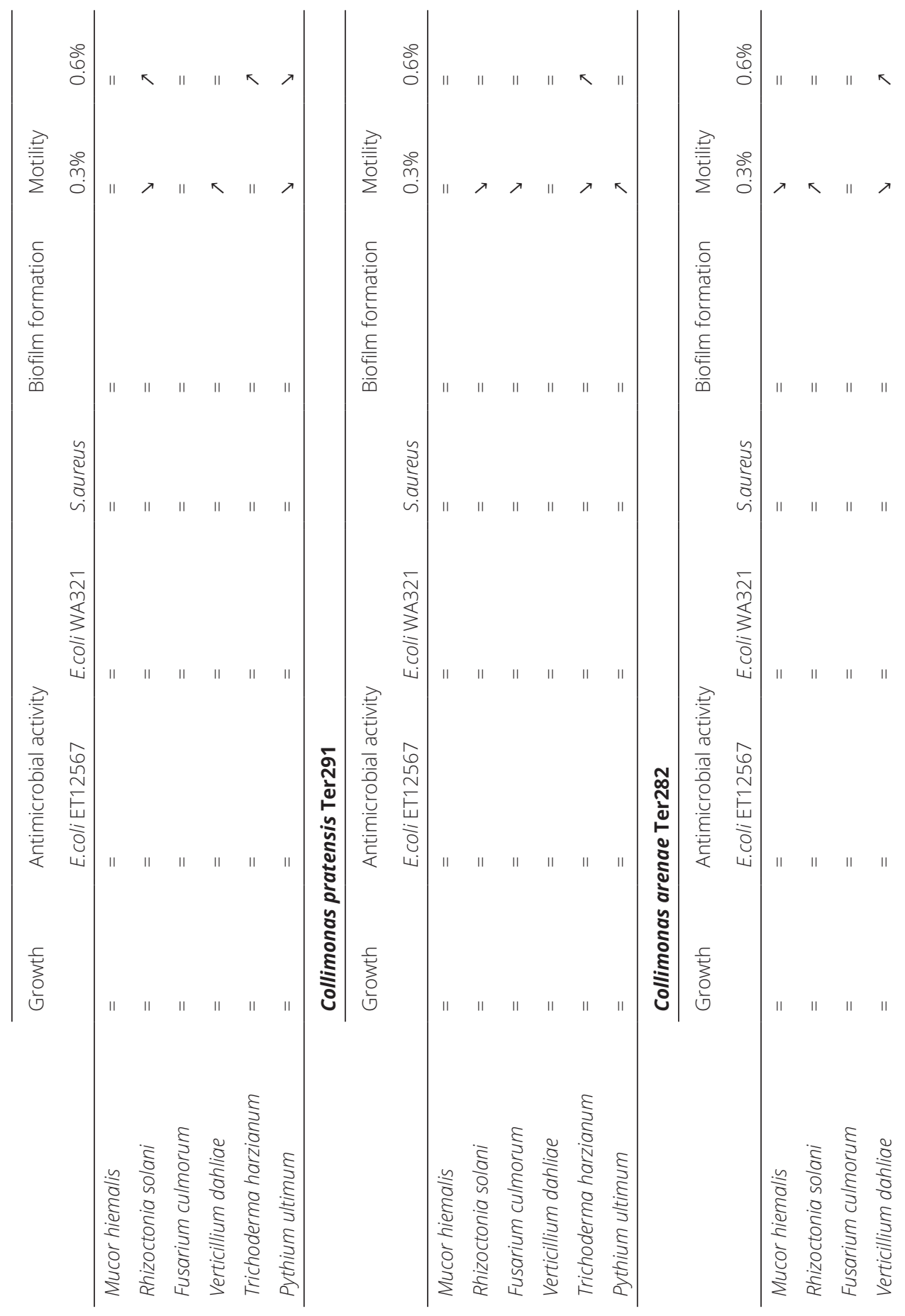




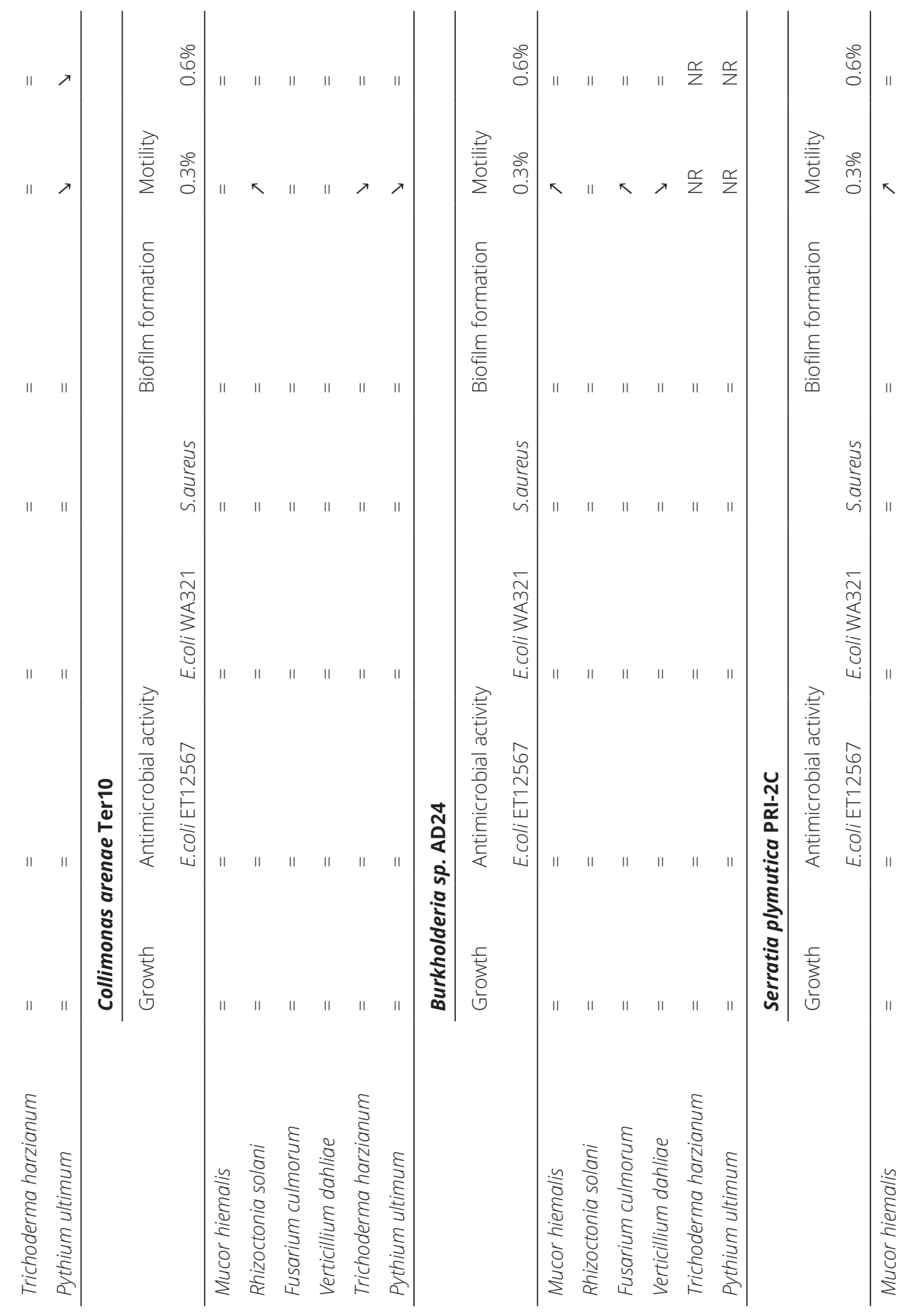




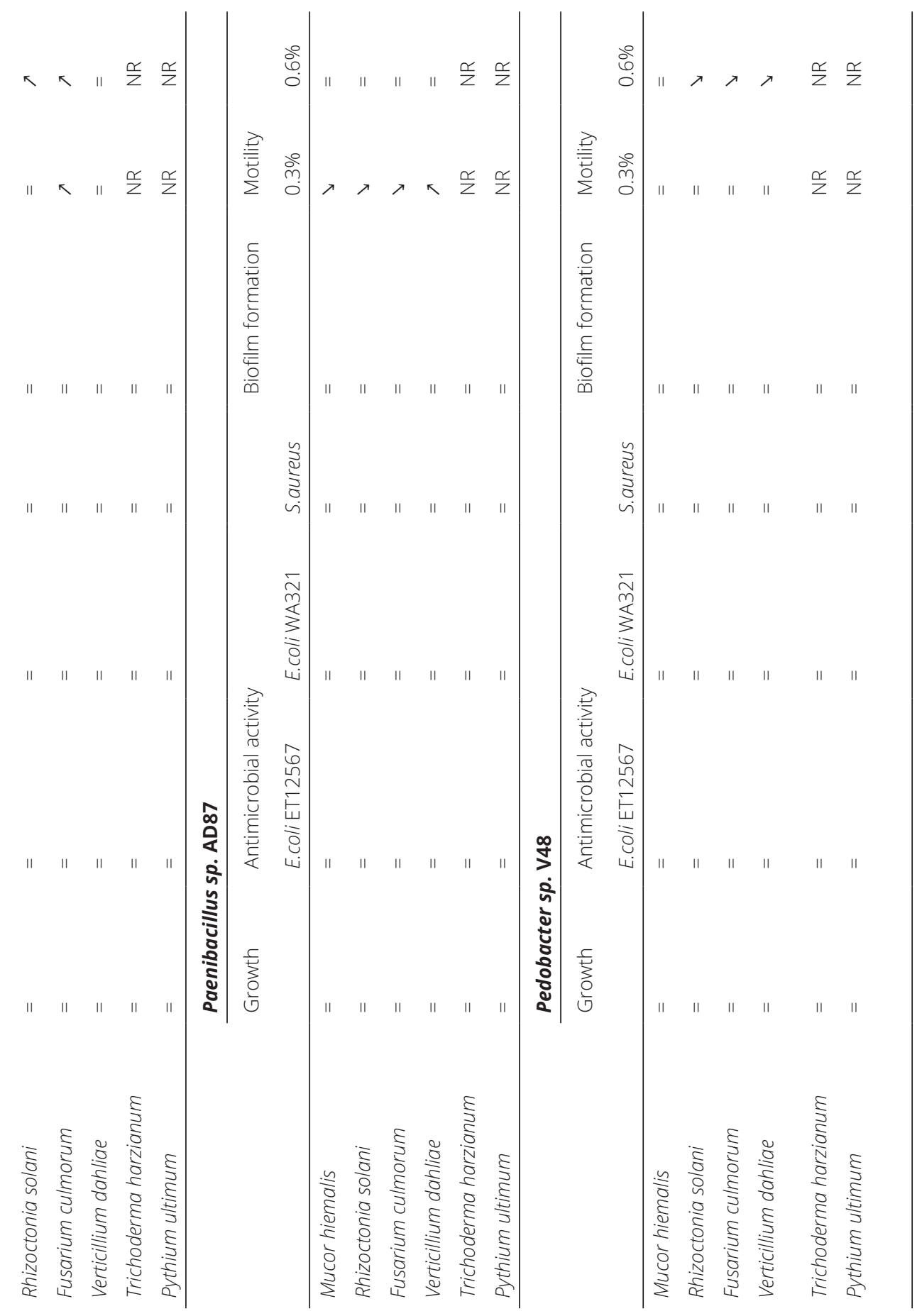




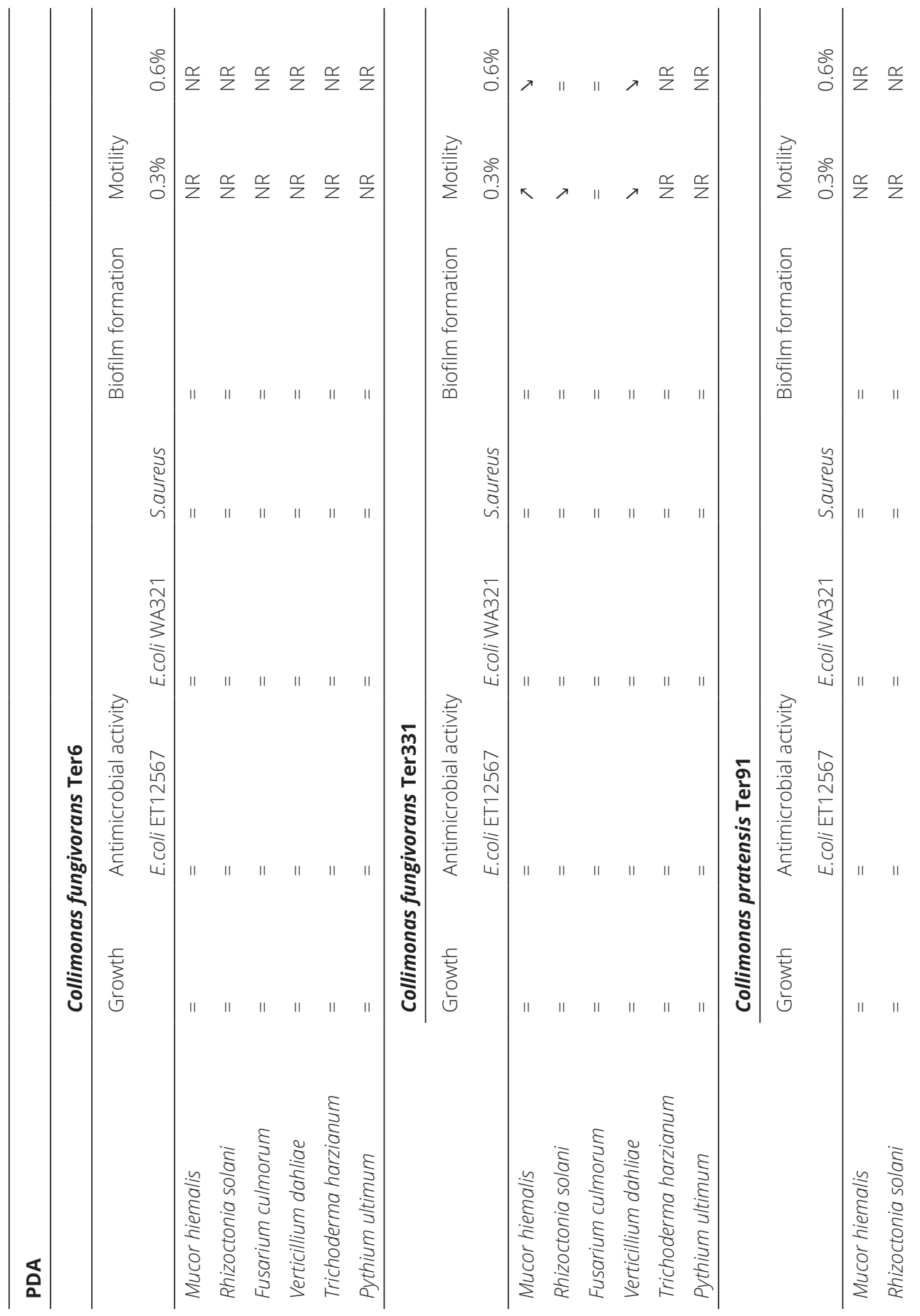




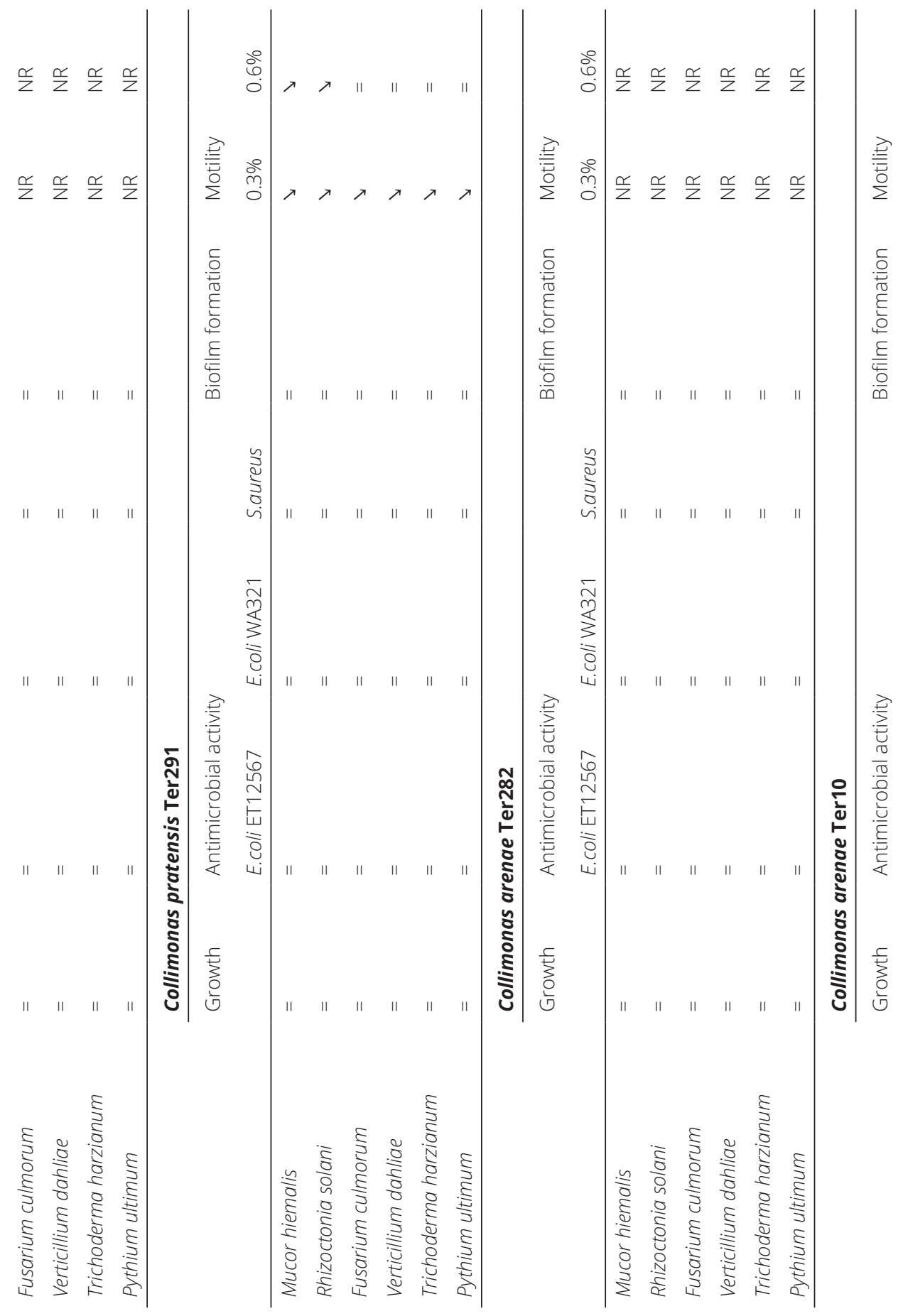




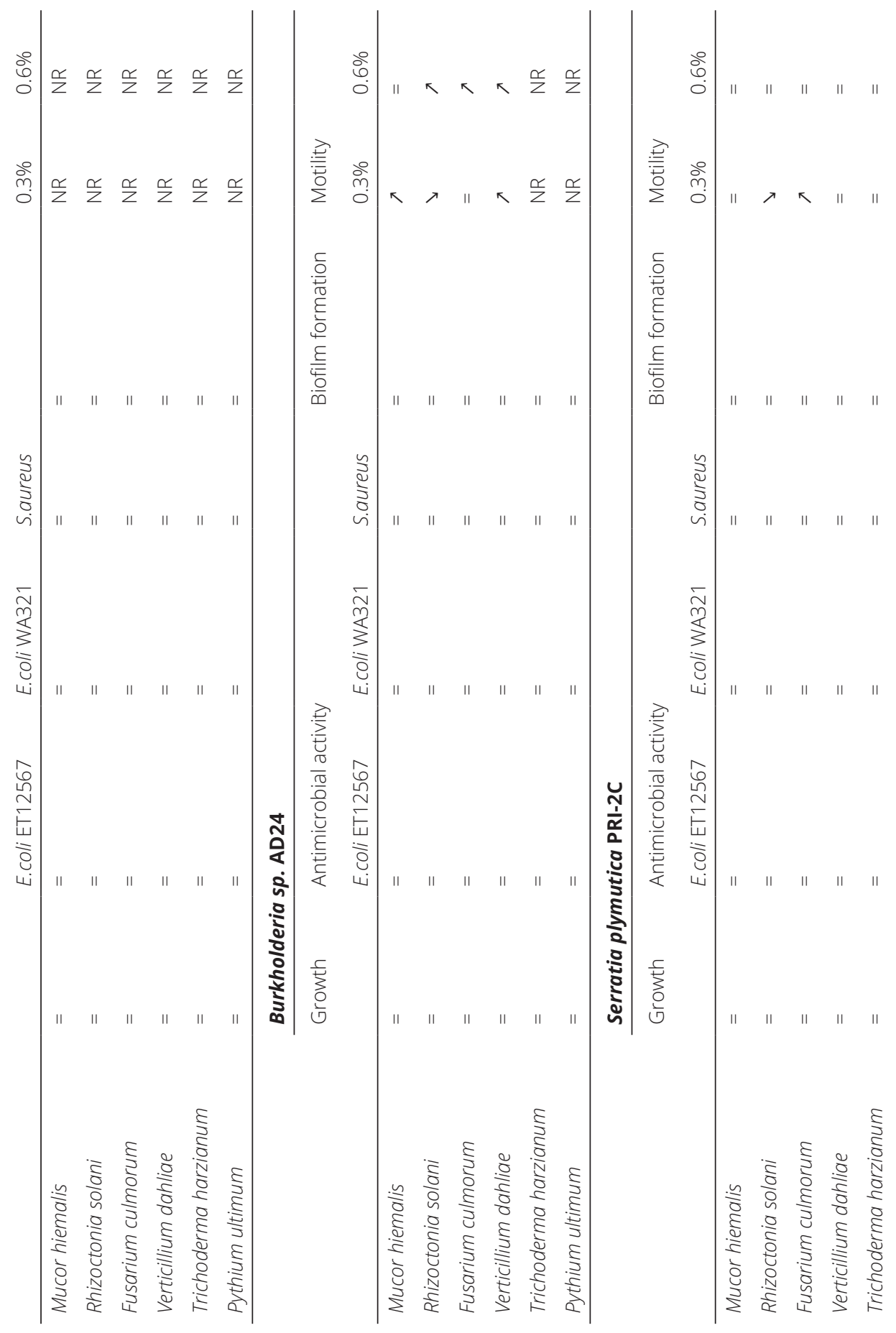




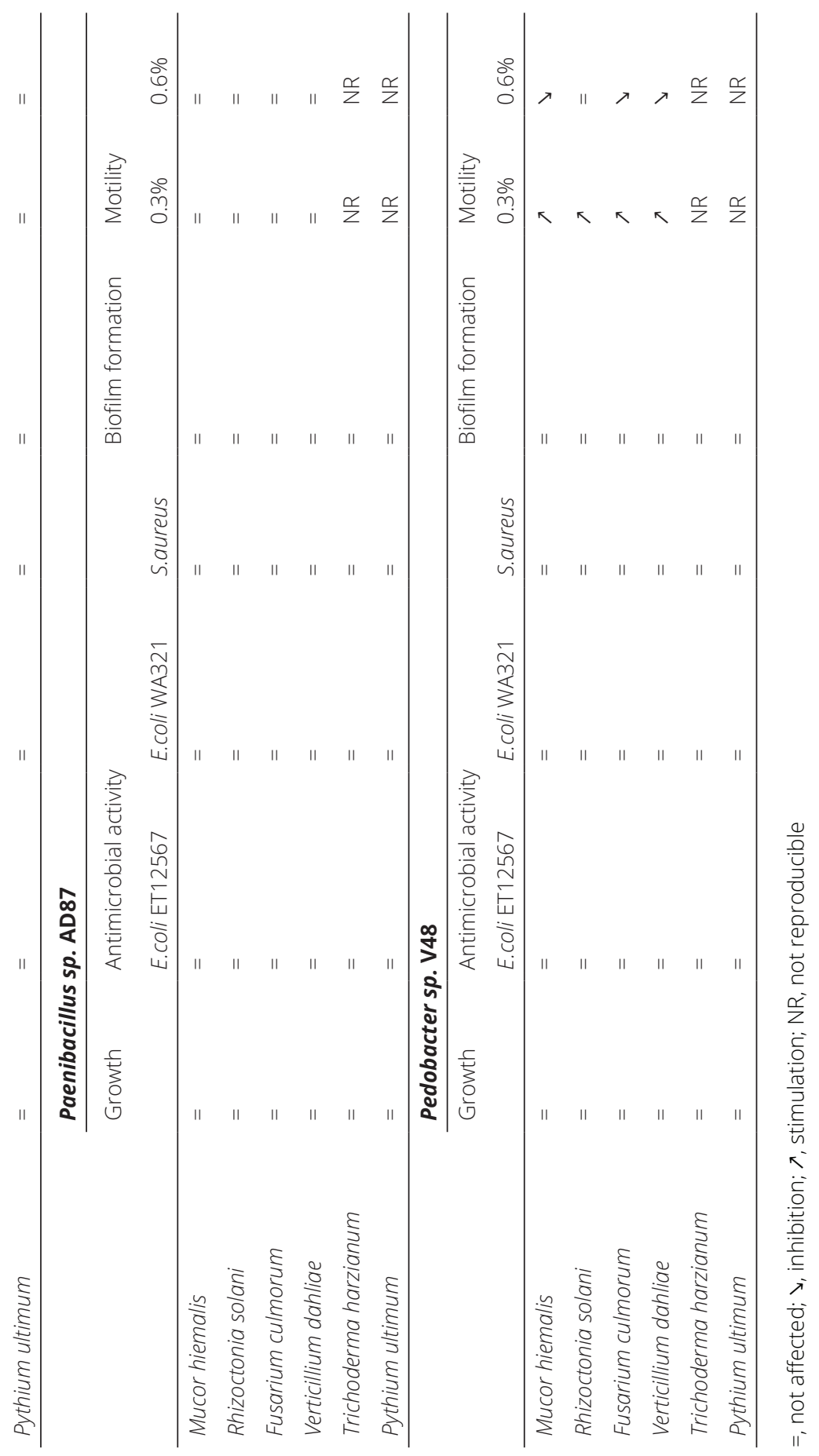


A

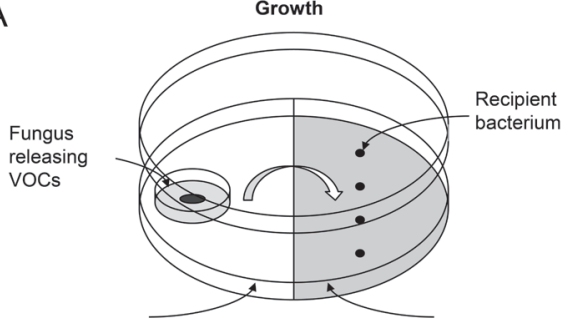

$1.5 \%$ WA+ARE or PDA medium $1.5 \%$ WA+ARE medium

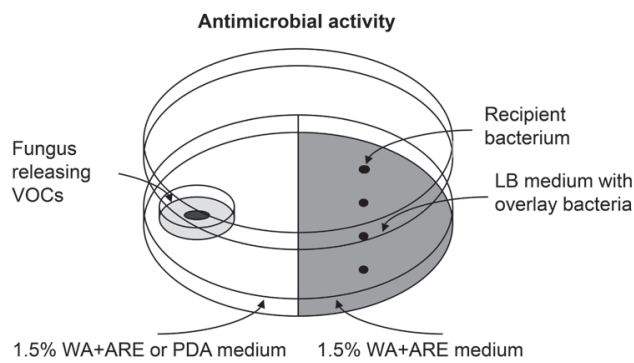

B

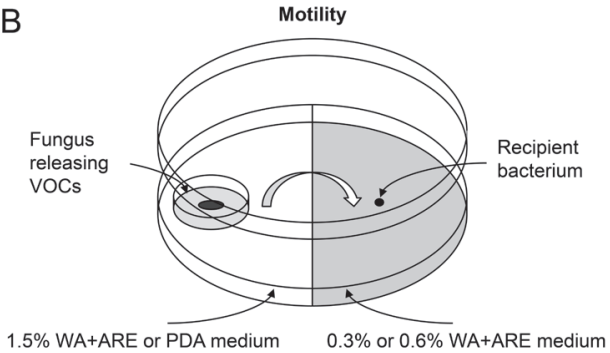

C

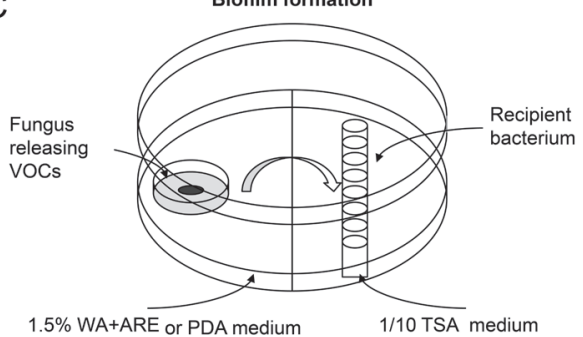

Fig. S1 / Variations of assays in double plate-within-a-plate system used to test the effect of fungal volatile compounds on bacterial growth and antimicrobial activity (A), motility (B) and biofilm formation (C) as described in section "Materials and Methods." 
A

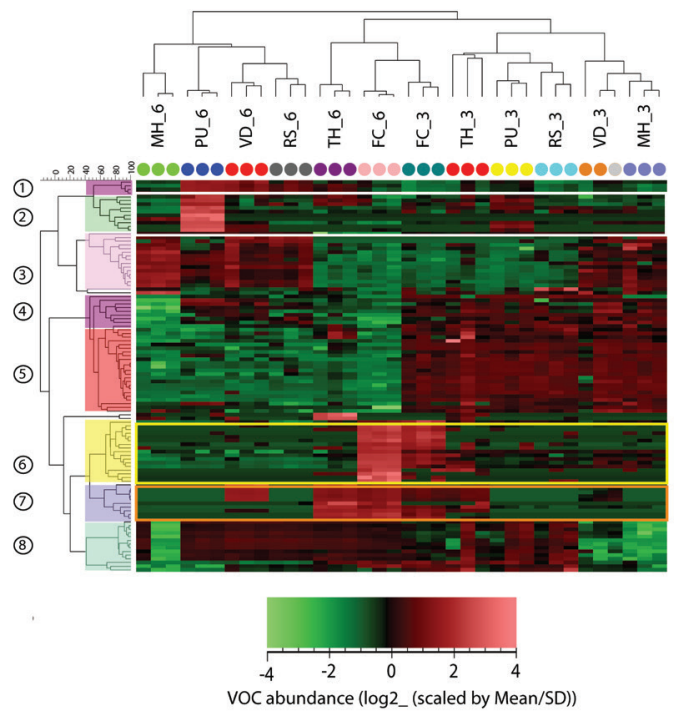

B
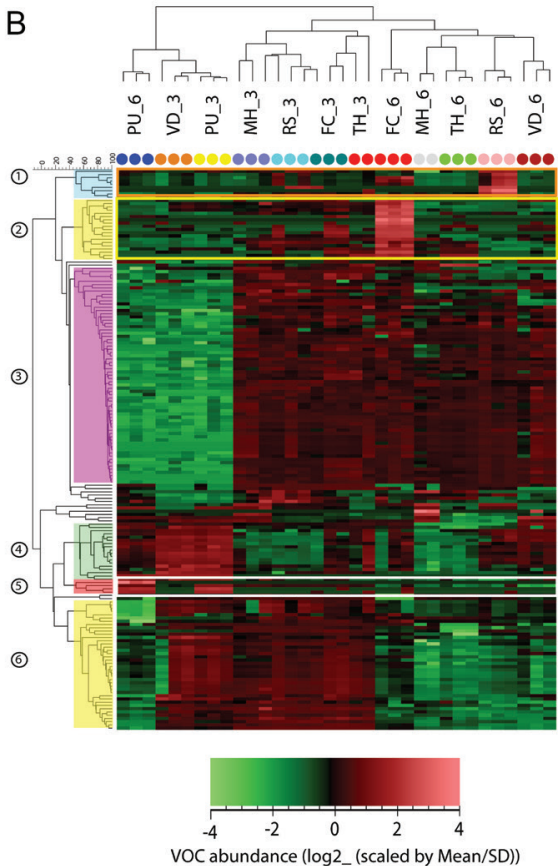

Fig. S2 I Hierarchical cluster analysis (HCA) of fungal and oomycetal strains based on volatiles that were significantly different ( $P<0.05$ with Bonferroni correction) in abundance between at least two strains when grown on water agar supplied with artificial root exudates (A) and on potato dextrose agar (B) at day 3 (early growth stage) and day 6 (late growth stage). MH, Mucor hiemalis; RS, Rhizoctonia solani; PU, Pythium ultimum; VD, Verticillium dahliae; FC, Fusarium culmorum; TH, Trichoderma harzianum; (C), Control (media). The color code below the Fig. indicates the abundance of the volatiles, which is log2transformed and scaled by Mean/SD. The boxes indicated in different colors discern the eight distinct clusters that determine the spatial separation of the samples in the PCA. 

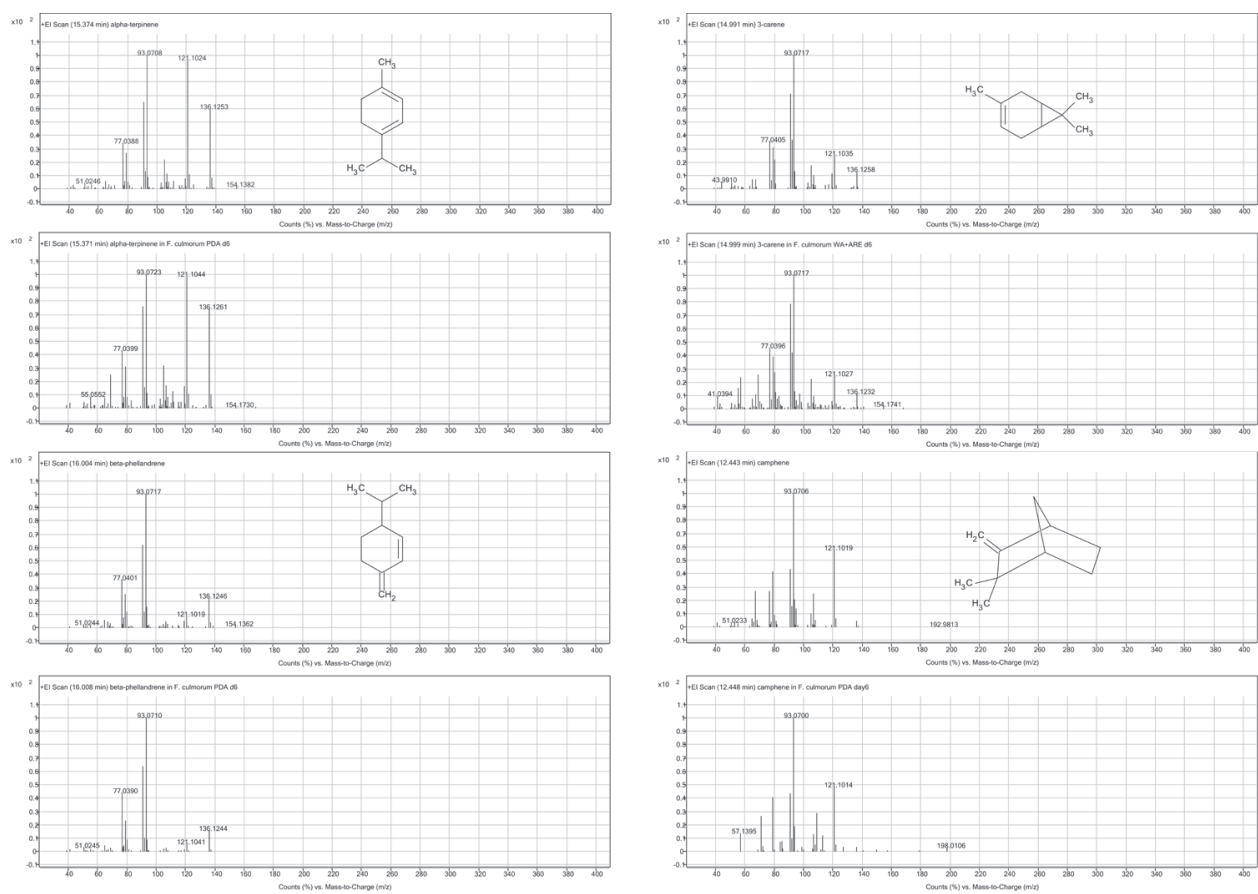

Fig. $\mathbf{S 3}$ / Comparison of mass spectra of the pure volatile compounds with those found in F. culmorum. 


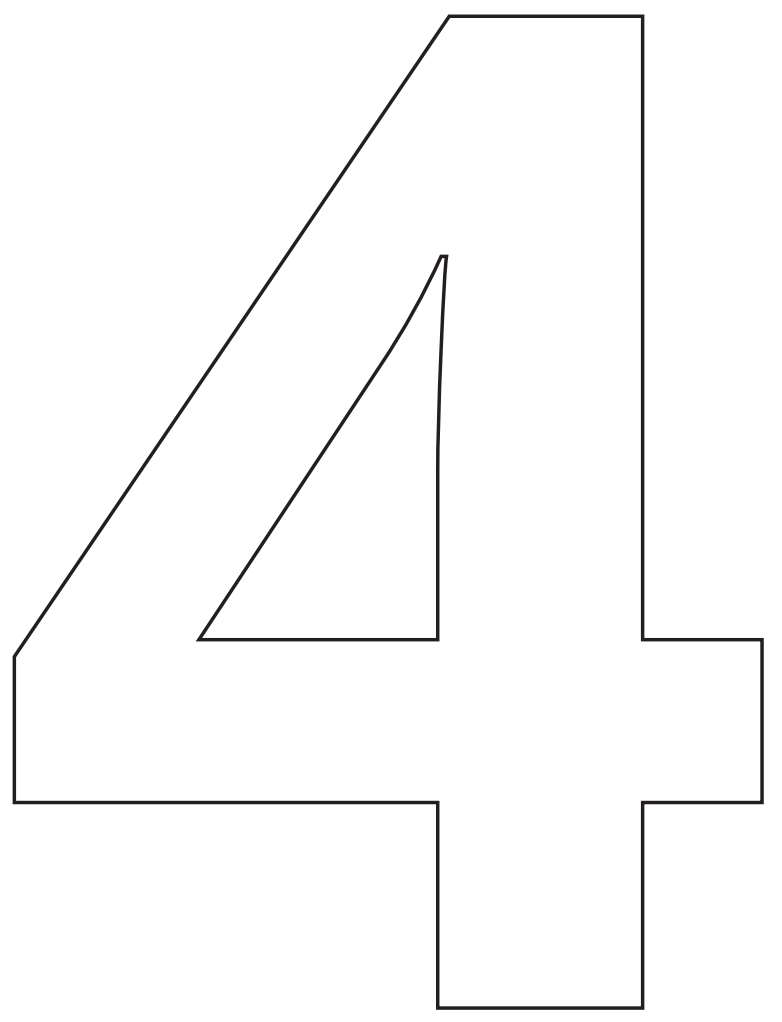

Fungal volatile compounds induce production of the secondary metabolite Sodorifen in Serratia plymuthica PRI-2C

Ruth Schmidt, Victor de Jager, Daniela Zühlke, Christian Wolff, Jörg Bernhardt, Katarina Cankar, Jules Beekwilder, Wilfred van ljcken, Frank Sleutels, Wietse de Boer, Katharina Riedel and Paolina Garbeva 



\section{Introduction}

Interactions and communication among organisms are central to understanding any ecosystem. The essential role of volatile organic compounds (VOCs) in the communication with other organisms, also known as infochemicals, has been acknowledged for more than 30 years (Dicke \& Sabelis, 1988). However, their ecological functions have been mainly studied for aboveground plant-plant and plant-insect interactions (Baldwin et al., 2006, Babikova et al., 2013). In recent years VOCs are becoming increasingly important in the field of microbial ecology. Due to their unique nature (low molecular mass, high vapor pressure, low boiling point and a lipophilic moiety) VOCs play important roles in the long-distance interaction and communication within the microbial world (Schmidt et al., 2015). In soil and in the rhizosphere VOCs readily diffuse under atmospheric pressure and travel throughout the air- and liquid-filled pockets of the soil (Morath et al., 2012, Schmidt et al., 2015).

Several soil-associated bacteria were shown to have positive effects on plant growth and resistance (Ryu et al., 2003, Blom et al., 2011) as well as to have the ability to control plant diseases via production of VOCs (Kai et al., 2007). Various studies have documented that VOCs can have diverse roles in the interaction between physically separated microorganisms ranging from infochemical molecules affecting the behavior, population dynamics and gene expression in responding microorganisms to interference competition tools suppressing or eliminating potential enemies (Effmert et al., 2012, Kim et al., 2013, Garbeva et al., 2014, Hol et al., 2015, Schmidt et al., 2015). Interestingly, most studies have only examined the role of bacterial VOCs and their effect on plants and fungi. However, the role and function of fungal VOCs on bacteria remains largely unknown. Only few studies demonstrated that the growth of some bacterial species was suppressed by fungal VOCs (Werner et al., 2016). For example, VOCs produced by the oyster mushroom Pleurotus ostreatus showed inhibitory effects on Bacillus cereus and Bacillus subtilis (Pauliuc, 2013). Another study by Lutz et al. (2004) demonstrated that VOCs emitted by $T$. atroviride increased the expression of a biocontrol gene ( $p h / A)$ of $P$. fluorescens.

Previously, we investigated the effect of fungal VOCs on the behavior of phylogenetically different soil bacteria (Schmidt et al., 2015). In these experiments we showed that VOCs emitted by several fungi lead to phenotypical responses in bacteria, for example, by inducing a change in bacterial motility (Schmidt et al., 
2015). We observed that the plant pathogenic fungus Fusarium culmorum produced a unique cluster of VOCs, consisting primarily of terpenes. When exposed to the VOCs emitted by this fungus, the rhizobacterium Serratia plymuthica PRI-2C responded with an induction of motility.

Itis plausiblethat insoil, microorganismssensechanges in their environments via shifts in VOCs blends and adapt their behavior accordingly (Garbeva et al., 2014). Although several studies indicated that VOCs can be used as signaling molecules in microbial inter-species interactions, the following questions remain unanswered: how are VOCs perceived as signals by the interacting microorganism and which regulatory genes and pathways are involved in the response?

To answer these questions, the rhizosphere isolate S. plymuthica PRI$2 \mathrm{C}$ was grown exposed or unexposed to VOCs emitted by F. culmorum and the bacterial transcriptome and proteome were analyzed under each situation to identify the molecular basis of the bacterial response to fungal VOCs. Further, metabolomics analysis and heterologous expression studies were performed to confirm the production of an unusual terpene compound involved in this bacterialfungal conversation.

\section{Methods}

\section{Strains and growth conditions}

Based on previous work (Schmidt et al., 2015) S. plymuthica PRI-2C and F. culmorum PV were selected for this study. The bacterial strain S. plymuthica PRI-2C was isolated from maize rhizosphere in the Netherlands (Garbeva et al., 2004), pre-cultured from frozen glycerol stocks on 0.1 Tryptic Soy Broth plates (TSB) (Garbeva \& de Boer, 2009) and grown for 3 days at $20^{\circ} \mathrm{C}$ before use. The fungal strain F. culmorum PV was isolated from a sandy dune soil in the Netherlands (De Boer et al., 1998), precultured on 0.5 Potato Dextrose Agar plates (PDA) (Fiddaman \& Rossall, 1993) and incubated for 6 days at $20^{\circ} \mathrm{C}$ before use.

\section{S. plymuthica PRI-2C genome sequencing}

Genomic DNA was extracted from S. plymuthica PRI-2C cells using the Qiagen MagAttract HMW DNA kit (Qiagen) and subjected to PacBio RS II sequencing at 
the Institute for Genome Sciences (IGS), Baltimore, Maryland, USA. PacBio RS II sequences were obtained from 1 SMRT cell. Sequences were filtered using SMRT Analysis server v2.3.0 with default settings and assembled using the RS_HGAP Assembly.3 (HGAP3) protocol, followed by a final Quiver correction using the RS_Resequencing protocol. The Illumina reads obtained from RNA-sequencing were filtered using Fastq MCF (Aronesty, 2011) with default settings and aligned against the contigs using BWA V0.7.12 (Li \& Durbin, 2009). The aligned reads were re-aligned with GATK V3.5.0 (McKenna et al., 2010)2010 to ensure correct indel calls. Contigs were corrected using the re-aligned reads with Pilon (Walker et al., 2014)2014. Singular contigs were checked with a custom script and overlapping ends were trimmed. The final circular contig was rearranged to start at the $d n a A$ gene in the forward direction. The sequences were annotated using Prokka 1.11 (Seemann, 2014). The accession number of the genome after submission to NCBI is CP015613.

\section{Interaction experiment between S. plymuthica PRI-2C and F. culmorum PV}

To investigate the response of S. plymuthica PRI-2C to volatiles emitted by $F$. culmorum PV, a plate-within-a-plate system was used (Fig. 1A). F. culmorum PV plugs ( $6 \mathrm{~mm}$ in diameter) were inoculated on a 0.5 PDA Petri dish $(3.5 \mathrm{~cm}$ ) that was placed into the partitioned Petri dish $(9 \mathrm{~cm})$ and incubated for 3 days at $20{ }^{\circ} \mathrm{C}$. On day 3 S. plymuthica PRI-2C inoculum, consisting of $50 \mu$ washed cell suspension in a $10 \mathrm{mM}$ phosphate buffer ( $\mathrm{pH}$ 6.5) and containing $10^{7} \mathrm{cells} / \mathrm{ml}$, was spread on 1.5\% water agar supplied with artificial root exudates (WA + ARE) (Schmidt et al., 2015) of the partitioned Petri dish. As control S. plymuthica PRI-2C was exposed to 0.5 PDA medium only. Plates were incubated at $20^{\circ} \mathrm{C}$. Total RNA and cytosolic proteins were extracted after $48 \mathrm{~h}$ (time point 1 ) and $72 \mathrm{~h}$ (time point 2). All experiments were performed in triplicates. 

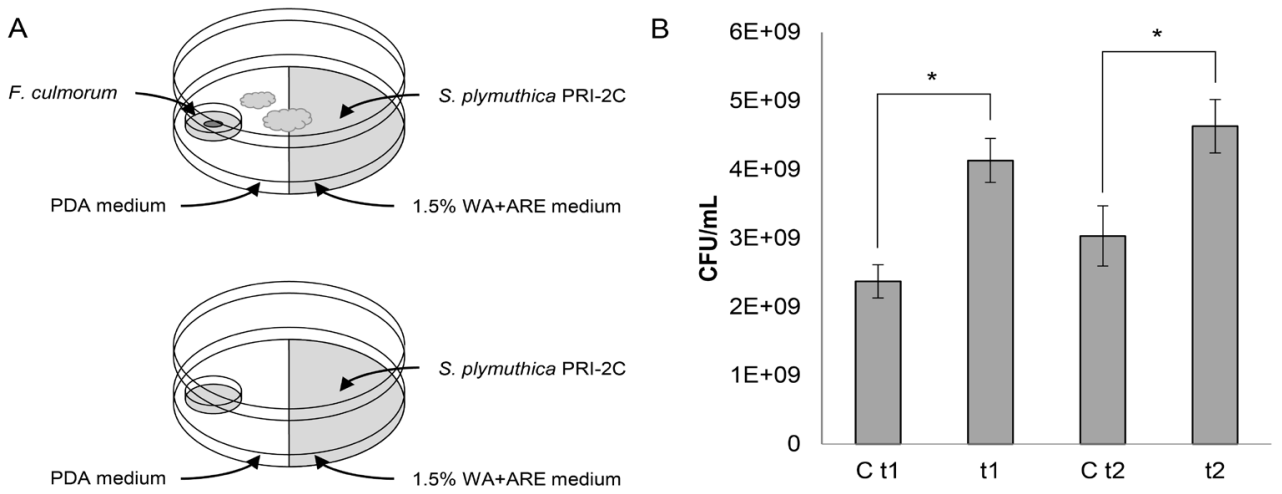

Fig. 1 I Overview of experimental setup and fungal VOC effect on bacterial growth. Schematic overview of the plate-within-a-plate system (A). Growth of S. plymuthica PRI-2C exposed to fungal VOCs after $48 \mathrm{~h}$ (t1) and $72 \mathrm{~h} \mathrm{(t2)} \mathrm{(B).} \mathrm{Controls} \mathrm{S.} \mathrm{plymuthica} \mathrm{PRI-2C} \mathrm{without} \mathrm{exposure} \mathrm{to} \mathrm{VOCs} \mathrm{after} 48 \mathrm{~h}$ (C t1) and $72 \mathrm{~h}$ (C t2). Error bars represent standard deviations between replicates $(n=3)$. Asterisk indicates statistical significance $(p<0.05)$ of each time point compared to the respective control.

To test the effect of fungal VOCs on the growth of S. plymuthica PRI-2C the CFU/ $\mathrm{ml}$ were determined after $48 \mathrm{~h}$ and $72 \mathrm{~h}$ (Fig. 1B). After scratching off the cells from the surface dilution series of each treatment were prepared in $10 \mathrm{mM}$ phosphate buffer ( $\mathrm{pH}$ 6.5). A volume of $100 \mu \mathrm{l}$ of each serial dilution was plated on $1 / 10$ th TSB plates in duplicates. The plates were incubated for three days at $20^{\circ} \mathrm{C}$. Bacterial enumeration was carried out on an aCOlyte Colony Counter (Meintrup DWS Laborgeräte $\mathrm{GmbH}$ ). A two-tailed tailed Student's t-test $(p<0.05)$ was used to evaluate the differences between two groups using SPSS (SPSS, Chicago, IL, USA). Results are shown as mean \pm S.D. Sample number $(n)$ represents biological replicates.

\section{RNA Extraction}

S. plymuthica PRI-2C cells were suspended in $2 \mathrm{ml}$ of sterile phosphate buffer and transferred to a double volume of RNAprotect Bacteria Reagent (Qiagen). The mixture was centrifuged at $14,000 \mathrm{rpm}$ for $1.5 \mathrm{~min}$ at $4^{\circ} \mathrm{C}$. The supernatant was discarded and the cell pellets were directly frozen in liquid $\mathrm{N}_{2}$ and stored at -80 ${ }^{\circ} \mathrm{C}$ until RNA extraction. RNA was extracted using the Aurum Total RNA Mini Kit (Bio-Rad), following the manufacturer's recommendations. Contaminating DNA was removed using the TURBO DNA-free Kit (Ambion). RNA concentrations and quality were measured on a NanoDrop Spectrophotometer (Isogen Life Science) and on a 
Fragmentanalyzer RNA 6000 Pico Chip (Agilent).

\section{RNA sequencing and differential expression analysis}

RNA sequencing was performed at Erasmus Center for Biomics, The Netherlands. RNA was depleted of rRNA using the Ribo-Zero rRNA Removal Bacteria Kit (Illumina). A sequencing library was constructed using the KAPA Stranded RNA-Seq Kit (Kapa Biosystems), and sequenced according to the Illumina TruSeq V3 protocol on the HiSeq2000 with a single read $50 \mathrm{bp}$ and $7 \mathrm{bp}$ index.

The obtained Illumina reads from the RNA-sequencing were filtered using Fasta MCF and aligned against the CDNA sequences of S. plymythica PRI-2C using Bowtie 2 (2.2.5) (Langmead \& Salzberg, 2012) with the following settings: ---no-mixed --no-discordant --gbar 1000 --end-to-end. Transcript abundance was estimated using RSEM V1.1.26 (Li \& Dewey, 2011) and differential expression between the treatments was analyzed using edgeR V3.2 (Robinson et al., 2010, Zhou et al., 2014). Differentially expressed genes (DEGs) were defined with a cutoff at log fold change of greater than 0.585 or less than -0.585, an FDR of less than 0.05. Data were filtered with a $p$-value of 0.001 (Table S2). Venn diagrams were produced using Venny (Oliveros). Functional prediction and assignment of genes to TIGRFAMs (Haft et al., 2013)2013 was accomplished by the in-house developed analysis pipeline 'Prophane 2.0' (http://www.prophane.de; Schneider et al. (2011). Voronoi treemaps were generated using Paver (Decodon, Greifswald, Germany, http://www.decodon. $\mathrm{com} /)$.

\section{Validation of RNA sequencing data}

Validation of RNA sequencing data was performed by Quantitative Real-Time PCR (qRT-PCR) analysis of a subset of differentially expressed genes related to terpene production, motility and a gene encoding for isocitrate lyase. Two housekeeping genes, rpo $B$ and $g y r B$, were used as internal standards (Table 1). All experiments were performed in triplicates. 
Table $\mathbf{1} /$ Primers used in $\mathrm{qPCR}$

\begin{tabular}{|c|c|c|c|}
\hline $\begin{array}{l}\text { Primer } \\
\text { code }\end{array}$ & Sequence $\left(5^{\prime}-3^{\prime}\right)$ of primers & Target & Reference \\
\hline 011535 _F & TGGACCGCATGACAAAGGAG & Putative terpene synthase & this study \\
\hline 011535 _R & TGAAGCGGTATAAGCCAGCC & Putative terpene synthase & this study \\
\hline $015265 \_F$ & TGTCTCAGGTTGACTCGCTG & Flagellin & this study \\
\hline 015265 _R & AGTCGGCATCCTGAATACGG & Flagellin & this study \\
\hline $015275_{-} F$ & AGTGGCGTAATGAGTGCCAG & Flagellar protein Flis & this study \\
\hline 015275 _R & ATATAGCCAGCCCTTTGCCG & Flagellar protein FliS & this study \\
\hline $011530 \_F$ & GCGCAATGGAAGAAGATCCG & Arabinose operon regulatory protein & this study \\
\hline $011530 \_R$ & GATTGCCGGTGATGAACTGAC & Arabinose operon regulatory protein & this study \\
\hline $023425 \_F$ & TACACCGATTTCTTCCTGCCG & Isocitrate lyase & this study \\
\hline 023425 _R & TCGCCTTCATCAGTTCGTAGG & Isocitrate lyase & this study \\
\hline $001325 \_F$ & GCCGAAAGGTGAAACCCAAC & $\begin{array}{l}\text { DNA-directed RNA polymerase } \\
\text { subunit beta RpoB }\end{array}$ & this study \\
\hline 001325 _R & TGCACGTCGATAACCGTACC & $\begin{array}{l}\text { DNA-directed RNA polymerase } \\
\text { subunit beta RpoB }\end{array}$ & this study \\
\hline 000020_F & GCACTACAGCGTGCAGAAAC & DNA gyrase subunit B GyrB & this study \\
\hline 000020_R & GGGTTGGCTGGTAGATCAGG & DNA gyrase subunit B GyrB & this study \\
\hline
\end{tabular}

First strand cDNA was synthesized from $2 \mu \mathrm{g}$ previously extracted total RNA with random hexamer primers using the SuperScript VILO cDNA Synthesis Kit (Invitrogen) according to the manufacturer's protocol. A volume of $4 \mu \mathrm{l}$ CDNA of each treatment was subjected to qRT-PCR using QuantiNova SYBR Green I PCR master mix (Qiagen) a Qiagen Research Rotor- Gene Q thermal cycler (Qiagen) with the following conditions: initial cycle $95^{\circ} \mathrm{C}$ for $2 \mathrm{~min}$, followed by 40 cycles of $95^{\circ} \mathrm{C}$ for $10 \mathrm{sec}$, and $60^{\circ} \mathrm{C}$ for $30 \mathrm{sec}$. Standard curves were established for each cDNA sample to calculate the expression values (Ct-value). Log fold changes in gene expression were determined using the $\Delta \Delta C$ t method (Livak \& Schmittgen, 2001), data were normalized against the two housekeeping genes ( $r p o B$ and gyrB), and compared to log fold changes obtained from RNA sequencing data (Fig. S1).

\section{Protein extraction and MS sample preparation}

S. plymuthica PRI-2C cells were suspended in $2 \mathrm{ml}$ of sterile phosphate buffer and centrifuged at $14,000 \mathrm{rpm}$ for $1.5 \mathrm{~min}$ at $4{ }^{\circ} \mathrm{C}$. Cytosolic proteins were extracted using a bead-beating protocol. Fractions were transferred into $2 \mathrm{ml}$ Eppendorf tubes with $0.25 \mathrm{ml}$ of $0.1 \mathrm{~mm}$ glass beads (BioSpec Products) and placed into FastPrep-24 lyser 
tubes (MP Biomedicals). Eight rounds of $30 \mathrm{~s}$ lysis at $6.5 \mathrm{~m} \mathrm{~s}-1$ followed by $5 \mathrm{~min}$ incubation on ice were used to lyse the cells. Afterwards, cell debris was removed by centrifugation; determination of cytosolic protein concentration was done using Roti ${ }^{\circledR}$-Nanoquant (Roth). Reduction of disulfide bonds with TCEP, alkylation of thiolgroups with iodoacetamide and subsequent in-solution digestion of proteins using trypsin was performed as described by Muntel et al. (2012). Desalting of peptides prior to mass spectrometry analysis using Stage tips was done according to the protocol by Rappsilber et al. (2007).

\section{Setup for label-free protein quantification (LC-IMSE) and data analysis}

The nanoACQUITYTM UPLCTM system (Waters) was used to separate and to introduce peptides into the Synapt G2 (Waters) mass spectrometer. Parameters for liquid chromatography and IMSE (MSE with ion mobility separation) were used as described previously (Muntel et al., 2014, Zuhlke et al., 2016).

LC-IMSE data were processed using PLGS v3.0.1 Processing parameters were set as follows: Chromatographic peak width and MS TOF resolution were set to automatic, lock mass charge 2 set to 785.8426 Da/e with a lock mass window of $0.25 \mathrm{Da}$, low energy threshold 200.0 counts, elevated energy threshold 20.0 counts, intensity threshold 750 counts. The data were searched against a randomized S. plymuthica PRI-2C database (version February 2016) with added laboratory contaminants and yeast ADH1 sequence (10,090 entries). For positive protein identification the following criteria had to be met: 1 fragment ion matched per peptide, 5 fragment ions matched per protein, 1 peptide matched per protein; 2 missed cleavages allowed, primary digest reagent: trypsin, fixed modification: carbamidomethylation C (+ 57.0215), variable modifications: deamidation N, Q (+ 0.9840), oxidation M (+ 15.9949), pyrrolidonecarboxylacid N-TERM (- 27.9949). The protein false discovery rate (FDR) was set to $5 \%$. For the final analysis only identifications based on at least two peptides were considered. In total 9 MS-runs per time point were conducted (3 biological replicates with 3 technical replicates each). For positive identification and reliable quantification, a replicate filter of $4 / 9$ was applied. This reduced the false discovery rate per sample to less than $0.7 \%$. Absolute protein quantification of identified proteins was performed using top3 peptide intensity with spiked-in yeast alcohol dehydrogenase (Waters; concentration of $50 \mathrm{fmol} / \mathrm{\mu l}$ sample) as a reference (Hi3 approach, Silva et al. (2006)). Data were corrected for detector saturation effects by implementing a correction factor as 
recently described (Zuhlke et al., 2016). To allow comparison between samples absolute protein amounts calculated by PLGS (given in fmol) were normalized (fmol/ng total protein).

Statistical analysis was done using MeV v4.8.1 (Saeed et al., 2003) for proteins that were present in at least four out of nine replicates. Hierarchical clustering and Student's t-test were performed with the following settings: unequal group variances were assumed (Welch approximation), $p$-values based on all permutation with $p=0.01$, significance determined by adjusted Bonferroni correction. Only proteins showing at least 1.5 fold changes in addition to statistical significance were considered for further analysis. So-called 'off/on' proteins needed to be detected in at least four replicates of one treatment and absent from all replicates of another treatment.

Functional prediction and assignment of proteins to TIGRFAMs (Haft et al., 2013)2013, respectively, was accomplished by the in-house developed analysis pipeline 'Prophane 2.0' (http://www.prophane.de; Schneider et al. (2011). Voronoi treemaps were generated using Paver (Decodon, Greifswald, Germany, http://www. decodon.com/). The mass spectrometry proteomics data have been deposited in the ProteomeXchange Consortium via the PRIDE partner repository (Vizcaino et al., 2014) with the dataset identifier PXD004819.

\section{Volatile trapping}

For the collection of volatiles produced during the interaction, partitioned glass Petri dishes with lids connected to a steel trap containing $150 \mathrm{mg}$ Tenax TA and 150 mg Carbopack B (Markes International Ltd., Llantrisant, UK) were used (Garbeva et al., 2014). F. culmorum PV was grown on 0.5 PDA at $20{ }^{\circ} \mathrm{C}$ for 3 days. On day 3 S. plymuthica PRI-2C inoculum, consisting of $50 \mu$ washed cell suspension in a $10 \mathrm{mM}$ phosphate buffer ( $\mathrm{pH}$ 6.5) and containing $10^{7} \mathrm{cells} / \mathrm{ml}$, was spread on 1.5\% WA + ARE. As control S. plymuthica PRI-2C was exposed to 0.5 PDA medium only. Plates were incubated for $48 \mathrm{~h}$ and $72 \mathrm{~h}$ at $20^{\circ} \mathrm{C}$. Volatiles were collected during $48 \mathrm{~h}$ and $72 \mathrm{~h}$ of incubation at $20^{\circ} \mathrm{C}$. All experiments were performed in triplicates. Traps were removed, capped and stored at $4{ }^{\circ} \mathrm{C}$ until analysis using GC-Q-TOF.

The trapped VOCs were desorbed from the traps using an automated thermo desorption unit (Unity TD-100, Markes International Ltd., Llantrisant, UK) 
at $210^{\circ} \mathrm{C}$ for $12 \mathrm{~min}$ (He flow $50 \mathrm{ml} / \mathrm{min}$ ) and trapped on a cold trap at $-10{ }^{\circ} \mathrm{C}$. The trapped volatiles were introduced into the GC-Q-TOF (model Agilent 7890B GC and the Agilent 7200A QTOF, Santa Clara, USA) by heating the cold trap for 3 min to 280 ${ }^{\circ} \mathrm{C}$ with split ratio set to 1:20. The column used was a $30 \mathrm{~mm} \times 0.25 \mathrm{~mm}$ ID RXI5MS, film thickness $0.25 \mu \mathrm{m}$ (Restek 13424-6850, Bellefonte, PA, USA). VOCs were detected by the MS operating at $70 \mathrm{eV}$ in El mode. Mass spectra were acquired in full scan mode (30-400 amu, 4 scans/s). MassHunter Qualitative Analysis Software V B.06.00 Build 6.0.633.0 (Agilent Technologies, Santa Clara, CA, USA) was used to control the instrument and for data acquisition and analysis. Mass-spectra were extracted with MassHunter Qualitative Analysis Software V B.06.00 Build 6.0.633.0 (Agilent Technologies, Santa Clara, USA) and compared with the data of the NIST NIST 2014 V2.20 library and the spectrum of sodorifen published in (von Reuss et al., 2010).

\section{Production of sodorifen in Escherichia coli}

PCR-generated DNA encompassing the complete coding sequence of the sodorifen synthase (SpSS, Q5A_011535) and the methyltransferase (SpMT, Q5A_011540) of S. plymuthica PRI-2C were inserted into MCS1 (BamHI, Notl) and MCS2 (Ndel, Kpnl) cloning sites of the expression vector pACYCDuet-1 (Novagen, $\mathrm{Cm}^{\mathrm{R}}$ ), respectively. The insertion was confirmed by colony PCR and sequencing. The constructed plasmids, pACYCDuet SpSS, pACYCDuet SPMT, PACYCDuet SpSS/SPMT and pACYCDuet empty, were transformed into chemically competent E. coli BL21 DE3 carrying the plasmid pMEV, which is a kanamycin-resistant version of the mevalonate pathway-expressing plasmid i-pBbA5c-MevT(CO)-MBIS(CO)-Nptll (Martin et al., 2003) (Addgene, Peralta-Yahya et al. (2011)), which was kindly supplied by Thamara Hendricks. Transformants were plated on LB plates supplemented with kanamycin $(50 \mu \mathrm{g} / \mathrm{ml})$, chloramphenicol $(50 \mu \mathrm{g} / \mathrm{ml})$ and $1 \%$ glucose. A starter culture of all 4 clones was grown overnight in $5 \mathrm{ml}$ LB medium supplemented with kanamycin (50 $\mu \mathrm{g} / \mathrm{ml})$, chloramphenicol $(50 \mu \mathrm{g} / \mathrm{ml})$ and $1 \%$ glucose at $37^{\circ} \mathrm{C}, 250 \mathrm{rpm} .500 \mu \mathrm{l}$ of the starter culture was inoculated into $50 \mathrm{ml}$ LB supplemented with kanamycin (50 $\mathrm{\mu g}$ / $\mathrm{ml}$ ) and chloramphenicol $(50 \mu \mathrm{g} / \mathrm{ml})$ in $250 \mathrm{ml}$ Erlenmeyer flasks. The culture was incubated at $37^{\circ} \mathrm{C}, 250 \mathrm{rpm}$ until the $\mathrm{OD}_{600}$ reached 0.4. At this point $100 \mu \mathrm{l}$ of each culture were spread on a glass Petri dish with $20 \mathrm{ml}$ LB agar supplemented with kanamycin $(50 \mu \mathrm{g} / \mathrm{ml})$, chloramphenicol $(50 \mu \mathrm{g} / \mathrm{ml})$ and $0.1 \mathrm{M} \mathrm{IPTG}$. Steel trap containing $150 \mathrm{mg}$ Tenax TA and $150 \mathrm{mg}$ Carbopack B were added into the lids and 
the plates were incubated at $25^{\circ} \mathrm{C}$ for $48 \mathrm{~h}$. All experiments were performed in duplicates. Traps were removed, capped and stored at $4{ }^{\circ} \mathrm{C}$ until analysis by using GC-Q-TOF. The traps were measured as described in the previous section with a split ratio set to 1:80. Mass-spectra were extracted with MassHunter Qualitative Analysis Software V B.06.00 Build 6.0.633.0 (Agilent Technologies, Santa Clara, USA) and compared with the data of the NIST NIST 2014 V2.20 library and the spectrum of sodorifen published in von Reuss et al. (2010) and Domik et al. (2016).

\section{Results}

\section{Genomic features of S. plymuthica PRI-2C}

The complete genome of S. plymuthica PRI-2C was sequenced to facilitate the interpretation of proteomics and transcriptomics data and to gain better insights into the bacterial perception and response to fungal VOCs. The complete genome of S. plymuthica PRI-2C is 5,464,425 bp in size. Annotation of the genome predicted 5,002 protein-coding genes, 88 tRNAs and 22 rRNAs (Accession number CP015613).

S. plymuthica PRI-2C harbors several gene clusters encoding for secondary metabolites. Based on in silico prediction using antiSMASH a total of 36 secondary metabolite gene clusters were identified. One of those gene clusters belonged to the class of terpenes, one to the class of arylpolyene-siderophore, one to the class of butyrolactones, one to nonribosomal peptide synthetase (nrps) -Type I polyketide synthase (t1 pks), one to homoserine lactone (hserlactones), one to others, two to nonribosomal peptide synthetase (nrps). Out of the remaining 28 gene clusters, four were identified as putative fatty acids, four as putative saccharides and twenty as non-identified putative gene clusters.

Important for microbial interactions and communication are signal transduction systems that enable bacteria to detect and respond to changes in the environment (Stock et al., 2000). We investigated the genome for signal transduction systems using the MIST2 database (Ulrich \& Zhulin, 2010). The genome of S. plymuthica PRI-2C encodes 428 one-component systems (1CSs) and 69 twocomponent systems (TCSS). Two extracytoplasmic function (ECF) sigma factors were found, which comprise the largest group among the $\sigma 70$ family (Ulrich et al., 2005). Additionally, 8 genes involved in chemotaxis systems were found (Table S1). 


\section{Effect of fungal VOCs on the growth of S. plymuthica PRI-2C}

After $48 \mathrm{~h}$ of exposure to fungal VOCs the cell density of S. plymuthica PRI-2C was $4.13 \times 10^{9} \mathrm{CFU} / \mathrm{ml}$ and after $72 \mathrm{~h}$ of exposure $4.63 \times 10^{9} \mathrm{CFU} / \mathrm{ml}$. We observed a significant increase in the growth of S. plymuthica PRI-2C exposed to VOCs after both time points as compared to S. plymuthica PRI-2C without exposure to VOCS (Fig. 1B).

\section{Global changes in the transcriptome and proteome of S. plymuthica PRI-2C in response to fungal VOCs}

To study the response of S. plymuthica PRI-2C to fungal VOCS, we investigated changes at the transcriptome and proteome level after $48 \mathrm{~h}$ (t1) and $72 \mathrm{~h}$ (t2) of exposure to F. culmorum VOCs. After $48 \mathrm{~h}$ of exposure to fungal VOCs 204 differentially expressed genes (DEGs) were identified (199 up- and 5 downregulated), while after $72 \mathrm{~h}$ of exposure only 6 DEGs were detected (5 up- and 1 down-regulated) (Fig. 2A, Table S2). Only 2 DEGs were unique for t2 and 4 DEGs were expressed at both times points (Fig. 2B). These 4 genes were Q5A_006040 (coding for the Pyrimidine-specific ribonucleoside hydrolase RihA), Q5A_018030 (coding for the putative HTH-type transcriptional regulator YahB), Q5A_003275 (coding for the UDP-N-acetylmuramoyl-L-alanyl-D-glutamate--2,6-diaminopimelate ligase) and Q5A_011375 (coding for a hypothetical protein). Most of the DEGs at t1 were assigned to functions involved in energy metabolism, transport and binding proteins, regulatory functions, cell envelope, cellular processes, protein synthesis and fate as well as unknown function. For t2 DEGs were assigned to functions involved in DNA metabolism, regulatory functions, cell envelope and unknown function (Fig. 2C, Table S2). 
A
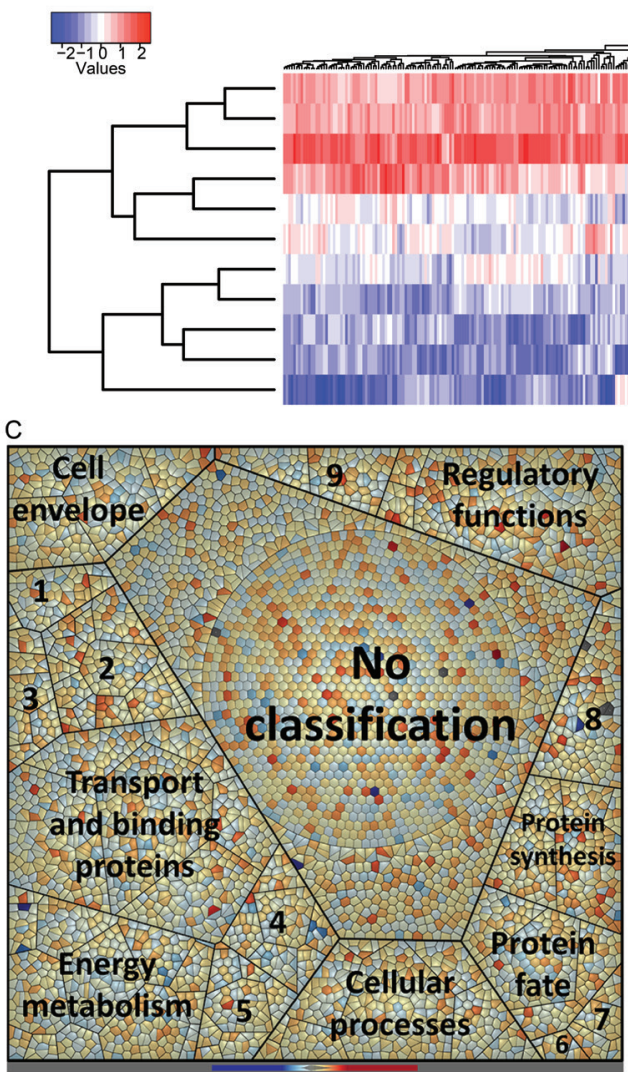

B

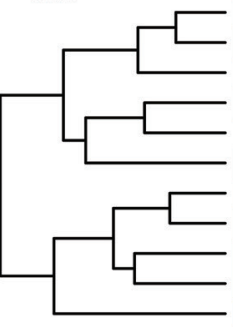

min

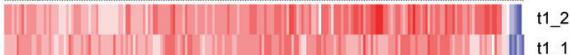

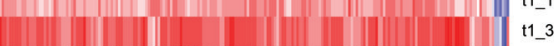

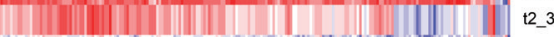

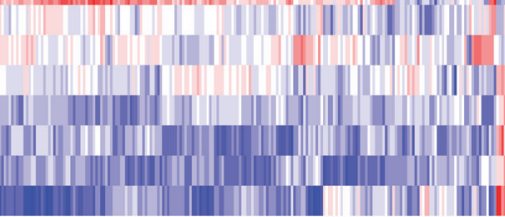

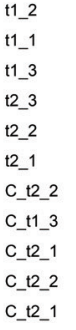
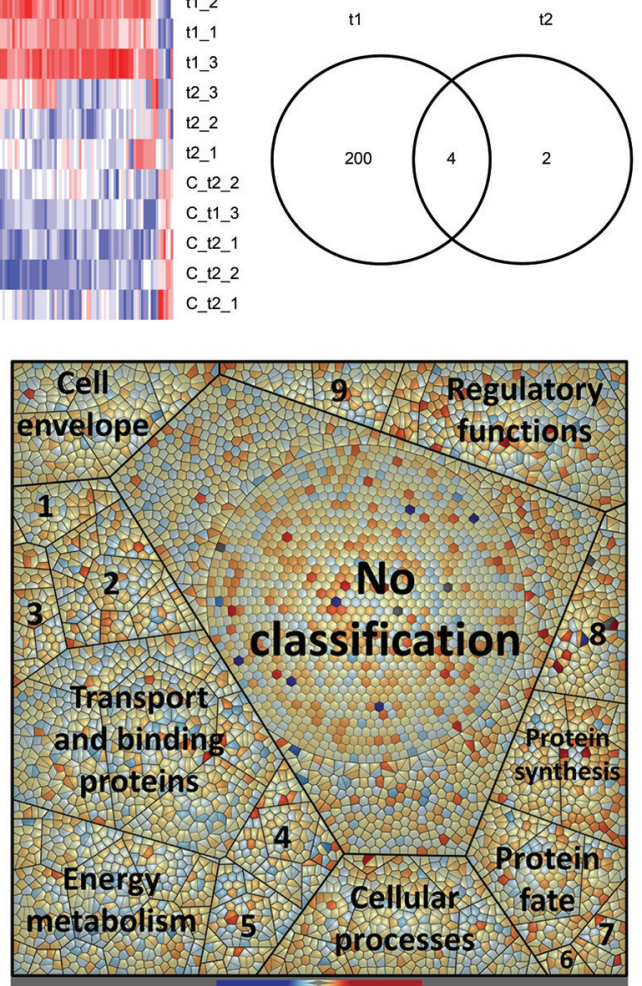

Fig. 2 I Changes in transcriptome of S. plymuthica PRI-2C in response to fungal VOCs. Heatmap of 206 DEGs with at least +/-1.5-fold changes and a p-value less than 0.001 as a cut-off (A). Hierarchical clustering was performed using the Pearson Correlation metric. Venn diagram of uniquely and commonly expressed DEGs at $\mathrm{t} 1$ and $\mathrm{t} 2$ (B). Voronoi treemaps visualizing changes in the transcriptome of S. plymuthica PRI-2C exposed to fungal VOCs at t1 (C left) and at t2 (C right) (c). Functional classification of genes was carried out by Prophane 2.0 and is based on TIGRFAMS. Red color indicates higher expression of the respective gene and blue color indicates lower expression as compared to the control. Each cell represents a single gene; functional classes are separated by thicker black lines. Numbers indicate the following classes: 1 - Fatty acid and phospholipid metabolism, 2 - Biosynthesis of cofactors, prosthetic groups, and carriers, 3 - Purines, pyrimidines, nucleosides, and nucleotides, 4 - Amino acid biosynthesis, 5 - Central intermediary metabolism, 6 - Mobile and extrachromosomal element functions, 7 - Transcription, 8 - DNA metabolism, 9 - Signal transduction.

On the proteome level absolute amounts for a total of 1,574 proteins were obtained by applying the LC-MSE approach(Silva et al., 2006) (Table S3). A large 
number of these proteins were assigned to functions associated with metabolic processes (584 proteins; e.g. biosynthesis of amino acids and cofactors; fatty acid, energy and central intermediary metabolism) and genetic information processing (311 proteins; e.g. protein synthesis and protein fate, DNA metabolism and transcription). Moreover, a high number of so far uncharacterized or unclassified proteins (448 proteins) were detected (Fig. 3, Table S3).

A

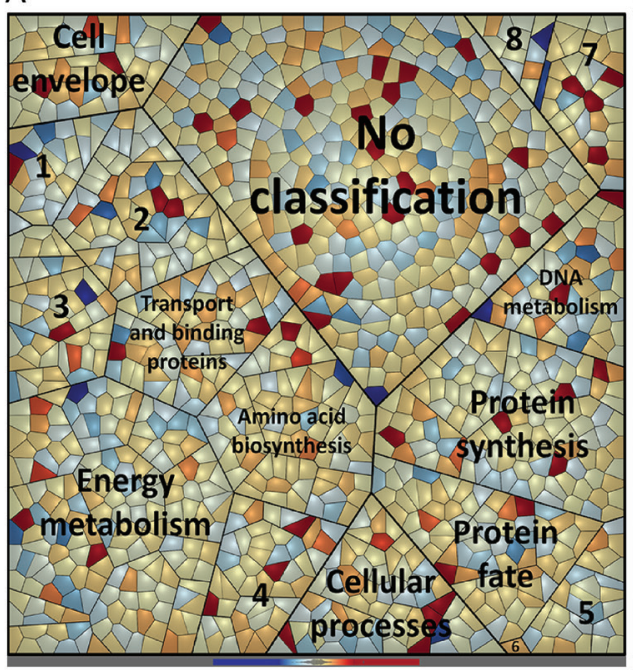

B

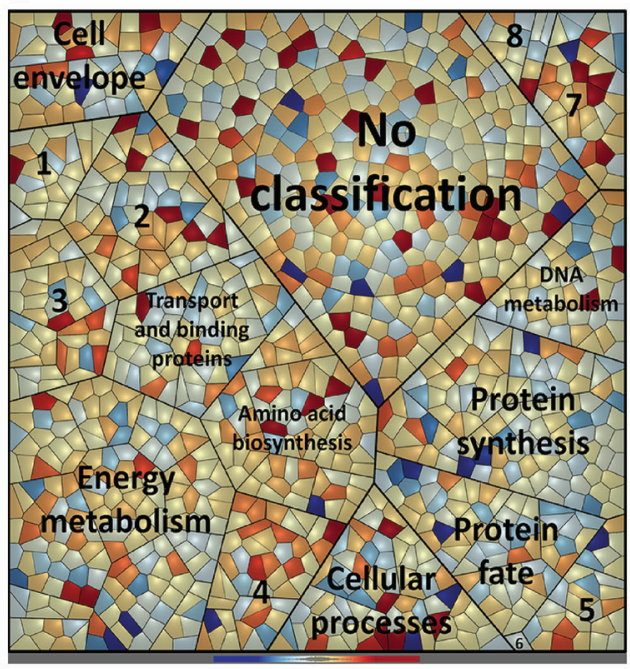

Fig. 3 I Voronoi treemaps visualizing changes in the proteome composition of S. plymuthica PRI$2 \mathrm{C}$ exposed to fungal VOCs. Quantitative expression values of S. plymuthica PRI-2C proteins at t1 of exposure (A) and at t2 (B). Functional classification of proteins was carried out by Prophane 2.0 and is based on TIGRFAMS. Red color indicates higher expression of the respective protein and blue color indicates lower expression as compared to the control. Each cell represents a single protein. Functional classes are separated by thicker black lines. Numbers indicate the following classes: 1 - Fatty acid and phospholipid metabolism, 2 - Biosynthesis of cofactors, prosthetic groups, and carriers, 3 - Purines, pyrimidines, nucleosides, and nucleotides, 4 - Central intermediary metabolism, 5 - Transcription, 6 Mobile and extrachromosomal element functions, 7 - Regulatory functions, 8 - Signal transduction.

Longer exposure (72h) to fungal VOCs led to higher number of proteins with a significantly changed amount as compared to the control. Overall 169 proteins were differentially expressed (significance determined by t-test and expression change of more than 1.5 fold) when exposed to fungal VOCs as compared to the non-exposed control (Table S3). At t1 51 differentially expressed proteins (DEPs) were identified (28 up- and 23 down-regulated) while at t2 124 DEPs were identified (78 up- and 46 down-regulated). For both time points mainly proteins involved in 
metabolic processes (amino acid biosynthesis, energy and central intermediary metabolism, biosynthesis of cofactors), signal transduction, transport and binding proteins and several uncharacterized proteins were found to be differentially expressed (Fig. 4, Table S3).

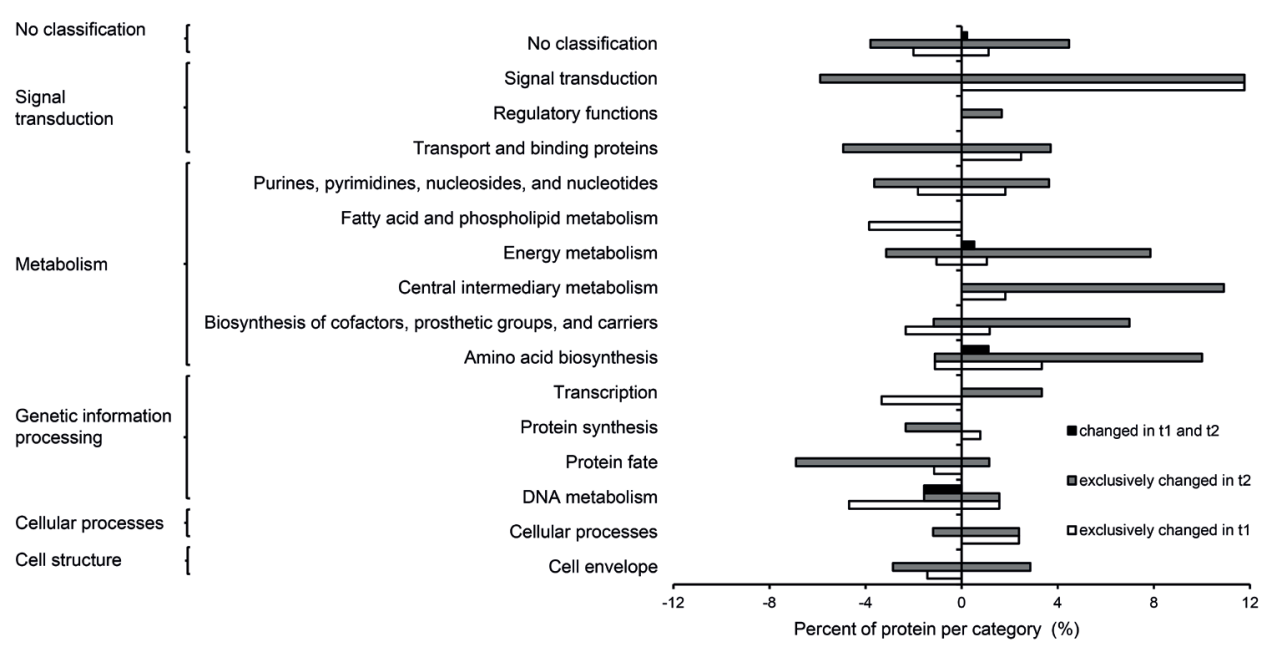

Fig. $\mathbf{4}$ / Impact of fungal volatiles on S. plymuthica PRI-2C protein content. The percentage of proteins with at least 1.5 fold change in relation to the number of identified proteins is depicted. Black bars indicate proteins with changed amount in both, early (t1, $48 \mathrm{~h}$ ) and late (t2, $72 \mathrm{~h}$ ) sampling points, grey bars indicate changed amounts on late sampling point, white bars indicate changed amount in early sampling point. Functional annotation is based on TIGRFAMS.

\section{Functional gene and protein categories affected by fungal VOCs}

\section{Differentially expressed genes and proteins related to chemotaxis and motility}

The expression of one gene, Q5A_015275 (coding for the flagellar protein FliS) relating to chemotaxis and motility was up-regulated at t1 when S. plymuthica PRI-2C was exposed to fungal VOCs (Table S2). One protein, Q5A_015505 (Methylaccepting chemotaxis protein IV) was identified at t1 (Table S3) and is known to act as a sensor detecting attractants and promoting bacterial movement towards suitable sites for colonization (Li et al., 2014).

\section{Differentially expressed genes and proteins related to signal transduction}

29 genes related to signal transduction were up-regulated in our transcriptomics dataset(Table S2). Due to the high number we focus here on genes coding for proteins 
related to small molecule interactions, protein interactions and two-component systems. Cyclic di-GMP phosphodiesterase YhjH (Q5A_007585) is known to be involved in regulating the levels of c-di-GMP that control cell motility (the flagella) and adhesion (adhesive curli fimbriae) (Pesavento et al., 2008). Phytochrome-like protein Cph2 (Q5A_015970) acts as a photoreceptor that perceives light signals (Park et al., 2000). The putative diguanylate cyclase AdrA (Q5A_005955) is an integral component of the membrane that induces cellulose synthesis, cell adherence to abiotic surfaces and swimming and swarming motility (Da Re \& Ghigo, 2006). Serine/threonine-protein kinase HipA (Q5A_018950) can inhibit cell growth and induces multidrug tolerance (Germain et al., 2013). Sensor histidine kinase Glrk (Q5A_019275) belongs to a two-component regulatory system that up-regulates transcription of sRNA (Yamamoto et al., 2005). The two-component system Nitrogen assimilation regulatory protein (Q5A_025300) was up-regulated in both, transcriptomics and proteomics data at t1. The expression of the corresponding gene is usually activated in response to nitrogen limitation (Feng \& Marzluf, 1998). Nitrate/nitrite response regulator protein NarL (Q5A_015065 and Q5A_018475) is involved in the modulate transcription of genes needed for anaerobic respiration with nitrate or nitrite as electron acceptors (Zhang et al., 2003). Transcriptional regulatory protein CitB (Q5A_019710) is involved in the expression of citratespecific fermentation genes (Yamamoto et al., 2008)2008.

In the proteomics dataset, the glucose-specific phosphotransferase enzyme IIA component (Q5A_018245) and nitrogen regulatory protein (Q5A_022765) were induced at t1 and t2 respectively (Table S3). Glucose-specific phosphotransferase enzyme IIA component is part of the sugar phosphotransferase system that is involved in glucose transport across the cell membrane (Saffen et al., 1987). The nitrogen regulatory protein is involved in the regulation of nitrogen utilization (Huergo et al., 2013). The sensor protein QseC (Q5A_021135) was only identified at $\mathrm{t} 2$, which is a member of the two-component regulatory system QseBC that activates the flagella regulon by activating transcription of FlhDC in E. coli (Sperandio et al., 2002). The PTS system fructose-specific EIIBC component (Q5A_017065), which is involved in the translocation of fructose across the membrane (Prior and Kornberg 1988), was repressed at $t 2$. 


\section{Differentially expressed genes and proteins related to the cell envelope}

Five genes coding for proteins related to the cell envelope were up-regulated at t1 of exposure to fungal VOCs (Table S2). The outer membrane protein W (Q5A_013980) is assumed to be involved in the protection of bacteria against various forms of environmental stress (Li et al., 2016). UDP-3-O-(3-hydroxymyristoyl)glucosamine $\mathrm{N}$-acyltransferase (Q5A_019950) is involved in the biosynthesis of lipid A of the outer membrane (Bartling \& Raetz, 2009). Fimbria A protein (Q5A_015665) is a major structural component of mannose-resistant fimbriae (Mizunoe et al., 1988)1988 and the putative fimbrial chaperone YfCS (Q5A_021485) is involved in pilus organization. At both $\mathrm{t} 1$ and $\mathrm{t2}$, the gene Q5A_003275 (UDP-N-acetylmuramoyl-L-alanyl-Dglutamate--2,6-diaminopimelate ligase) was up-regulated, which is involved in the biosynthesis of bacterial cell-wall peptidoglycan (McGroty et al., 2013).

At t2 on the proteomics level, two proteins, UDP-N-acetylmuramate--Lalanyl-gamma-D-glutamyl-meso-2,6-diaminoheptandioate ligase (Q5A_001880) and UDP-N-acetylglucosamine 1-carboxyvinyltransferase (Q5A_022690) were present in higher levels. These proteins are involved in the peptidoglycan recycling pathway, part of cell wall biogenesis (Mengin-Lecreulx et al., 1996, Skarzynski et al., 1996).

The expression of three proteins was reduced at $\mathrm{t} 1$ and $\mathrm{t} 2$. The methioninebinding lipoprotein MetQ (Q5A_007015) was reduced at t1 and is involved in the transport of methionine (Gal et al., 2002). D-alanyl-D-alanine carboxypeptidase DacA (Q5A_005980) was reduced at t2 and plays a role in the pathway peptidoglycan biosynthesis. Mannose-1-phosphate guanylyltransferase 1 (Q5A_007950) was repressed at $\mathrm{t} 2$ and is involved in the synthesis of GDP-alpha-D-mannose.

\section{Differentially expressed genes and proteins related to energy metabolism}

Three genes, Q5A_001620 (fumarate reductase subunit D), Q5A_011355 (Glutathione S-transferase GST-6.0) and Q5A_024300 (Putative 3-oxopropanoate dehydrogenase) were upregulated at $\mathrm{t1}$ (Table S2). The latter one is involved in the degradation of beta-alanine (Yao et al., 2011).

On the transcriptome level, alpha-acetolactate decarboxylase(Q5A_018170) and isocitrate lyase (Q5A_023425) were downregulated at t1, while the latter one was found in higher amounts on the proteome level at t1. Isocitrate lyase catalyzes 
the bypass of decarboxylation steps of the TCA cycle (glyoxylate shunt). The Glyoxylate shunt is upregulated under different stress conditions, e.g. oxidative stress and antibiotic stress.

\section{Differentially expressed genes and proteins related to biosynthesis of natural products}

We observed an upregulation of gene expression of Q5A_011535, which encodes a putative terpene synthase, at 11 of exposure to VOCs (Table S2). The corresponding protein showed the highest induction rate of all proteins in the proteomics dataset at t2 (Table S3). This gene is part of a 3-gene cluster comprising genes from the DXP pathway for isoprenoid biosynthesis (DXP synthase Q5A_011545), isopentenyl diphosphate isomerase (Idi, Q5A_011550) and a methyl transferase (Q5A_011540).

\section{Volatile analysis of S. plymuthica PRI-2C and F. culmorum PV interaction and heterologous expression of sodorifen}

As in both, transcriptomics and proteomics datasets, the putative terpene synthase (Q5A_011535) was upregulated in response to F. culmorum VOCs we further investigated the production of the terpene compound encoded by this gene. By comparing the VOC emission of S. plymuthica PRI-2C exposed to F. culmorum VOCS to S. plymuthica PRI-2C unexposed to fungal VOCs, we observed one particular compound produced in higher amounts in response to fungal VOCs. This compound was identified as sodorifen $\left(\mathrm{C}_{16} \mathrm{H}_{26} ;\right.$ mass=218.2033; RT=28.62) (Fig. 5). 


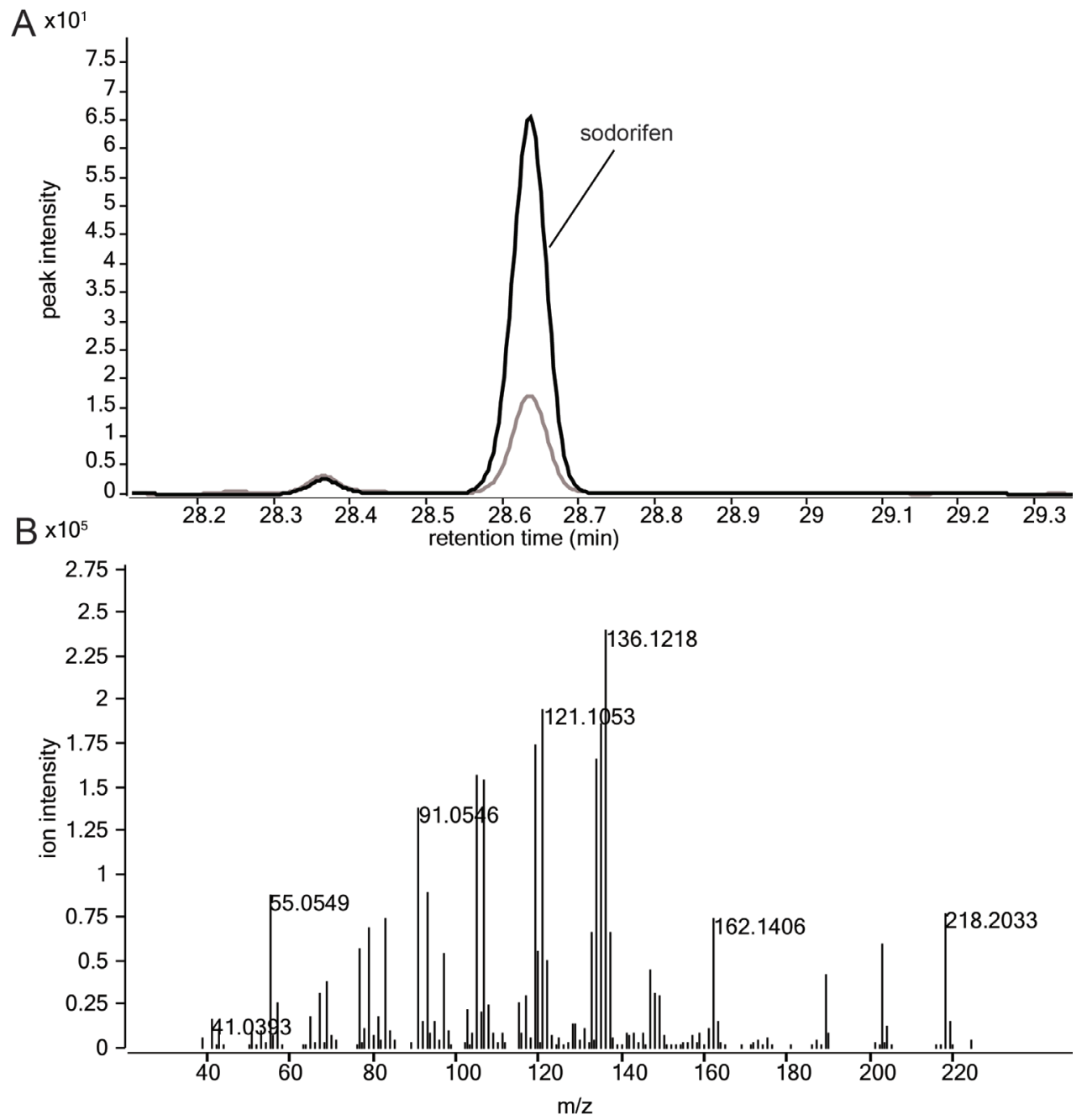

Fig. 5 I Volatile spectra of the sodorifen peak at RT 28.6 produced by S. plymuthica PRI-2C exposed to fungal VOCs (black) and by S. plyumthica without exposure to fungal VOCs (grey) (A) and mass spectrum of sodorifen produced by S. plymuthica PRI-2C (B)

Sodorifen is a recently discovered terpene which is likely derived from farnesyl pyrophosphate (FPP), but would need an additional methylation to reach its final number of carbons (C16). To confirm that the terpene synthase and methyltransferase can mediate the production of sodorifen, the synthase and methyltransferase genes Q5A_011535 and Q5A_011540 were expressed in an E. 
coli strain overproducing FPP. Upon co-expression of both genes, formation of sodorifen was detected in the headspace of $E$. coli cultures, confirming involvement of these genes in the synthesis of sodorifen (Fig. 6A). No products were detected upon expression of either Q5A_011535 (now called SpSS) or Q5A_011540 (now called SpMT) alone. In addition to sodorifen we observed a cluster of other unidentified terpene compounds following the peak of sodorifen (Fig. 6B). This suggests that the terpene synthase is a multi-product terpene synthase that produces a set of sodorifen like terpenes simultaneously. Indeed a number of these peaks were also found in the headspace of Serratia (Fig. 6B).

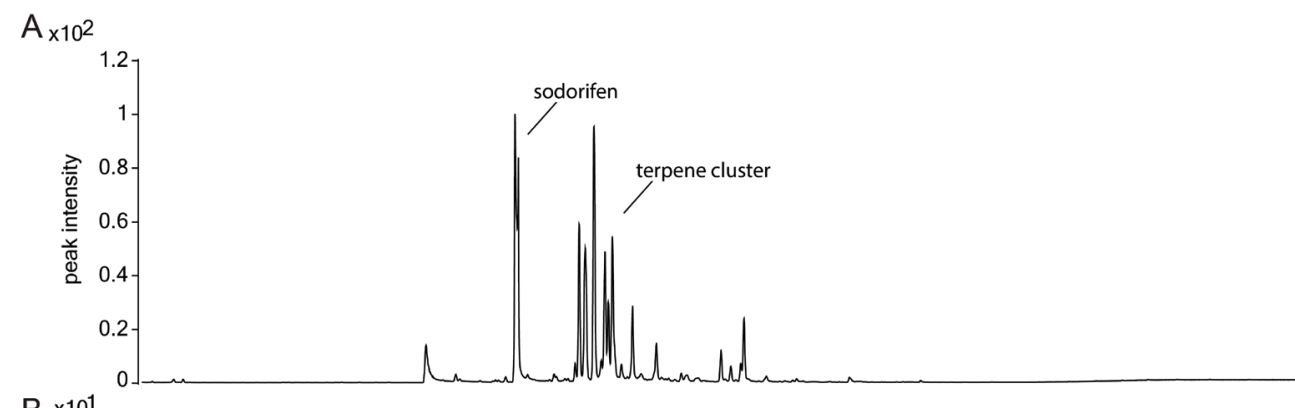

$B \times 10^{-1}$
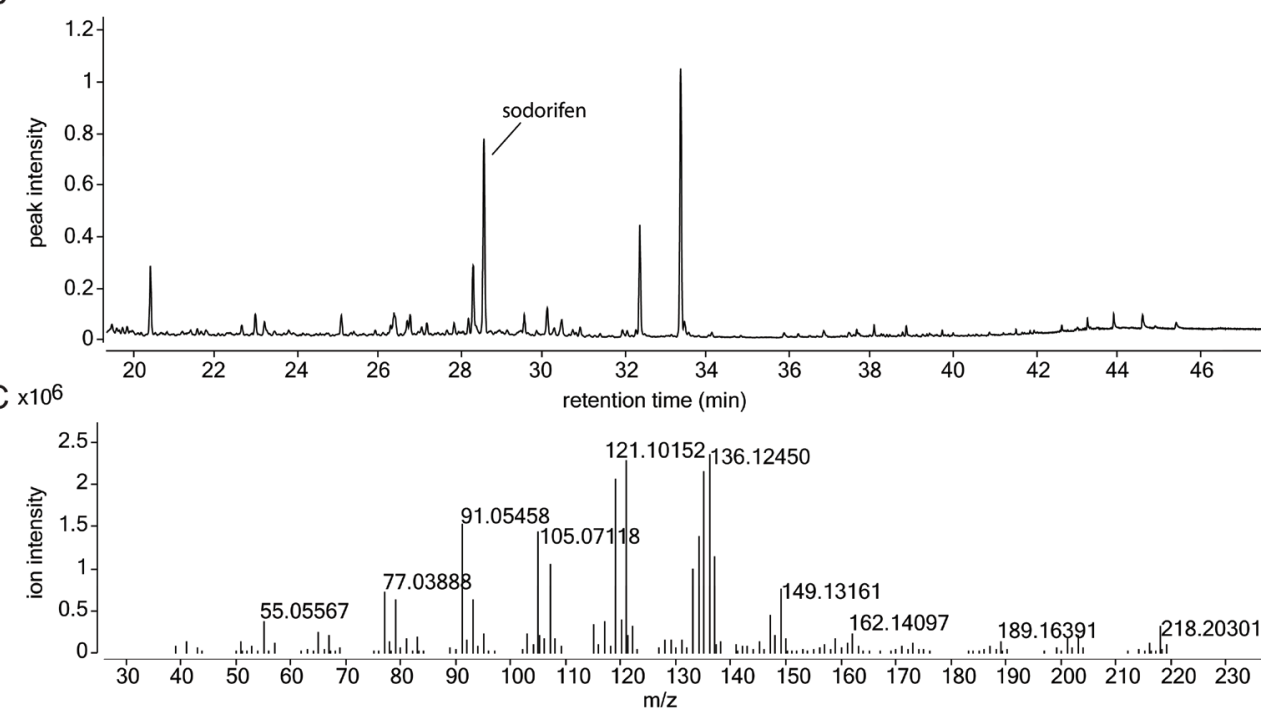

Fig. $\mathbf{6}$ / Volatile spectra of E. coli producing sodorifen (A) in comparison to S. plymuthica PRI-2C (B) and mass spectrum of sodorifen produced by E. coli co- expressing SpSS and SpMT (C). 


\section{Comparison of the sodorifen cluster in other bacterial species}

We compared the presence of the sodorifen cluster with other bacterial species using antiSMASH (Medema et al., 2011). The results show that the sodorifen cluster of S. plymuthica PRI-2C is present in several closely related isolates, including the originally described sodorifen producer S. plymuthica 4Rx13 (Fig. 7).

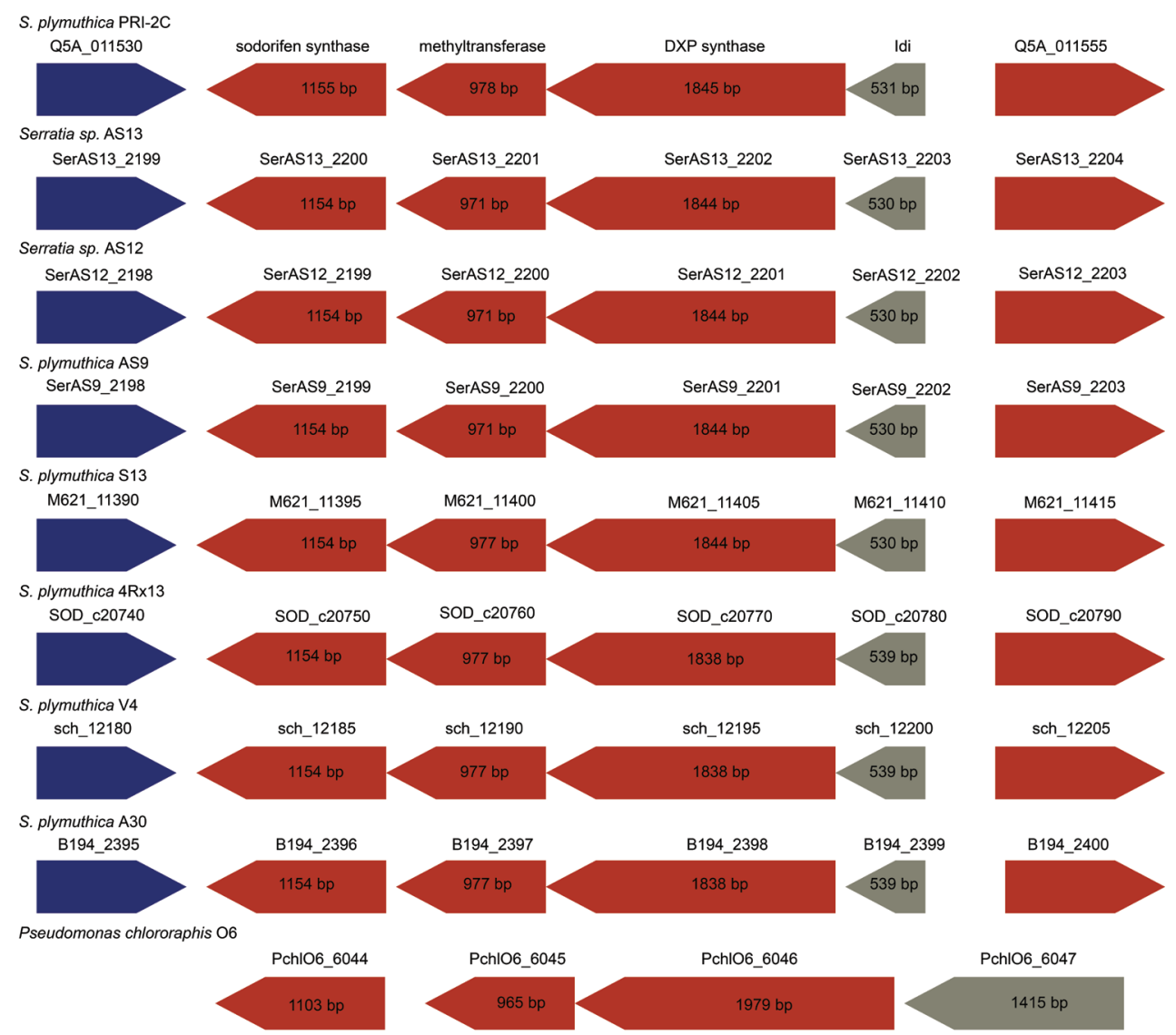

Fig. 7 / Comparison of the sodorifen cluster within other bacteria. The sodorifen synthase (Q5A_011535) forms a cluster with three additional genes, methyltransferase (Q5A_011540), 1-deoxy-D-xylulose-5phosphate synthase (Q5A_011545) and isopentenyl-diphosphate delta-isomerase (Q5A_011550), which are positioned in the sense direction within the genome of S. plymuthica PRI-2C. The cluster is bordered by genes that are in antisense orientation. Red indicates biosynthesis genes; blue indicates regulatory genes and grey indicates other genes. 


\section{Discussion}

The ability of microorganisms to communicate is well recognized, however, deciphering the language and the mechanism of communication remains a challenging task. In the recent years several independent studies, including our own, indicated VOCs are ideal info-chemicals for mediating both, short- and longdistance interactions (Effmert et al., 2012, Garbeva et al., 2014, Schmidt et al., 2015). Recently, we have shown that VOCs produced by Fusarium culmorum affect the motility of the rhizosphere isolate Serratia plymuthica without affecting bacterial cell numbers (Schmidt et al., 2015). In the current study we applied both transcriptomic and proteomic approaches to understand the mechanisms underlying bacterial perception and responses to fungal VOCs. Integrated data of our complementary Omics-approach are summarized in Fig. 8.

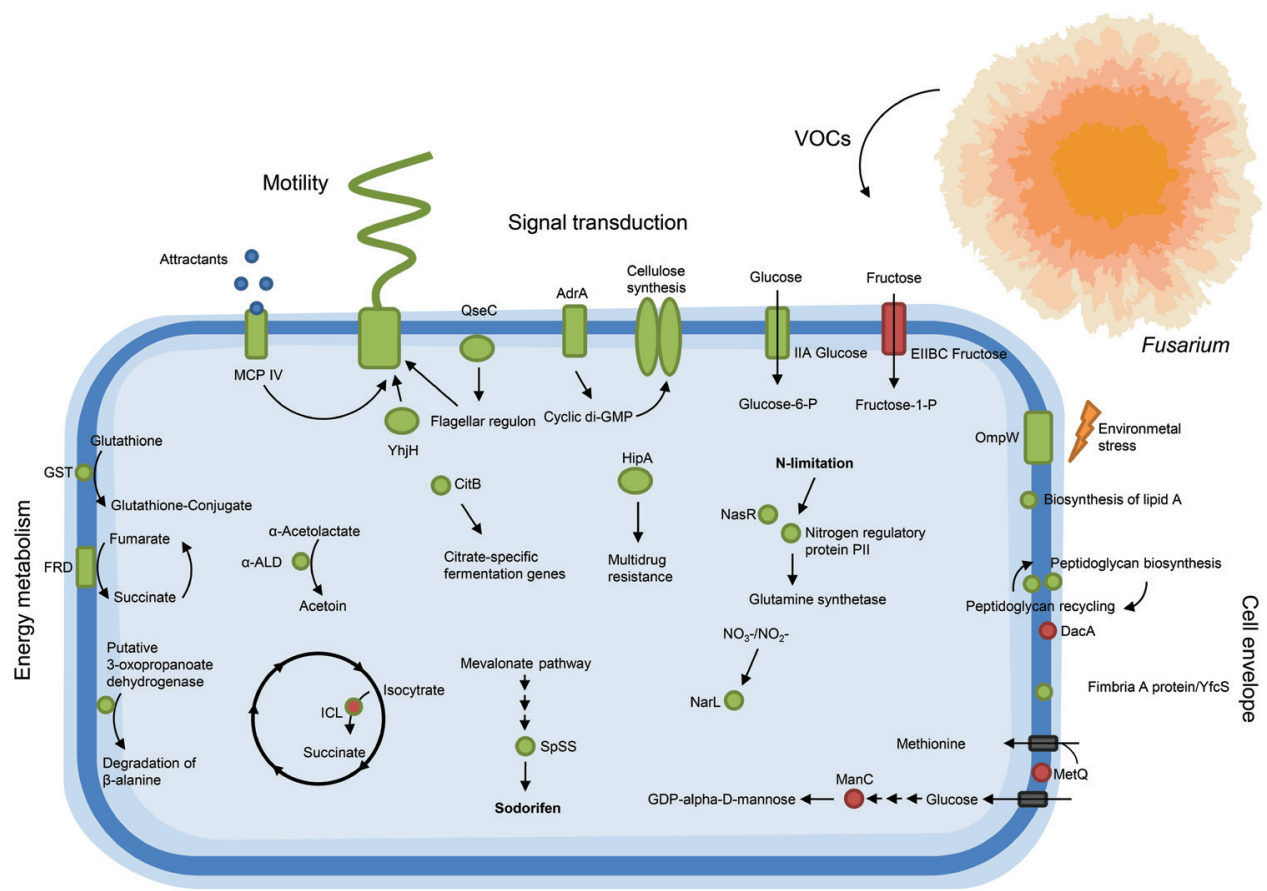

Serratia plymuthica

Biosynthesis of natural products

Fig. 8 / Schematic overview of the most important changes in S. plymuthica PRI-2C transcriptome and proteome in response to fungal VOCs. The overview includes genes and proteins involved in major pathways that have been detected in this analysis and genes and proteins whose amounts were either decreased (red) or increased (green) after exposure to fungal VOCs. 
Access to the complete genomic sequence of an organism is crucial for a reliable interpretation of functional omics data as it enables to correctly identify differentially expressed genes and proteins. Hence, prior to the transcriptomic and proteomic analysis the complete genome of S. plymuthica PRI-2C was sequenced and submitted to NCBI. Both transcriptomics and proteomics approaches identified several genes and proteins related to motility, which is in line with the induced $S$. plymuthica motility in response to fungal VOCs. Among these, Flis was described as a flagellin-specific chaperone that prevents premature polymerization of flagellins by facilitating its export (Galeva et al., 2014). Fimbria A protein was identified in S. marcescens as a major structural component of mannose-resistant fimbriae involved in cell-adhesion (Mizunoe et al., 1988)1988. On the proteomics level the methylaccepting chemotaxis protein IV was identified, which acts as chemotactic-signal sensor that detects attractants and promotes bacterial movement towards suitable sites for colonization (Baer et al., 2014). Bacterial motility is an important strategy to move toward a favorable stimulus or away from an unfavorable one (Alexandre, 2015). We speculate that the increased motility in S. plymuthica in response to F. culmorum VOCs indicates that fungal VOCs act either as chemo attractants or repellents in these fungal-bacterial interactions.

Next to motility our results revealed several differentially expressed genes and proteins involved in signal transduction. These genes and proteins were involved in the regulation of motility, cell growth, and multidrug tolerance or were part of the two-component system, involved in the regulation of nutrient uptake across the membrane and in the regulation of nutrient limitation. Based on the fact that VOCs modulate antimicrobial activity, Kim et al. (2013) hypothesized that VOCs could modulate multidrug resistance. Indeed, the study by Kovac et al. (2015) showed that the monoterpene (-)-a-pinene modulates antibiotic resistance in Campylobacter jejuni and decreased membrane permeability, which was related to the increased expression of the outer membrane protein product of the omp50 gene. The authors speculated that the up-regulation of omp50 is a strategy for bacterial adaptation contributing to decreased membrane permeability and decreased antimicrobial influx. Overexpression of the observed HipA protein has been described to lead to multidrug tolerance in E. coli (Coerreia et al 2006). Thus, we suggest that fungal VOCs may as well modulate multidrug tolerance in bacteria.

Some VOCs might serve as energy source under nutrient limited conditions 
and stimulate the growth of other organisms (Garbeva et al., 2014, Tyc et al., 2015). Recently Tyc et al. (2015) revealed that bacterial response to VOCs can vary from growth stimulation to growth inhibition. In this study, we observed a significant increase in S. plymuthica PRI-2C cell numbers when exposed to fungal VOCs. It seems plausible that bacteria take up carbon- or nitrogen-containing volatiles produced by other microorganisms in their surroundings.

In addition we observed a number of upregulated genes and proteins relating to cell envelope biogenesis. Induction of these genes and proteins could imply that the bacterial cells activate membrane biosynthetic pathways when exposed to certain fungal VOCs that causing damages in the bacterial cell wall and thus require repair. Similar findings have been observed by Yung et al. (2016) when E. coli was exposed to $\mathrm{N}$-methyl-2-pyrrolidone. However, at the same time several genes and proteins relating to cell wall/envelope biogenesis were repressed.

In a previous study we observed that $F$. culmorum emits a cluster of terpenes (Schmidt et al., 2015). The interaction between terpenes and the bacterial cell of pathogenic bacteria wall has been mainly described as increased permeability of the cell membrane leading to a loss of microbial viability (Trombetta et al., 2005, Nazzaro et al., 2013). Kovac et al. (2015) described a concentration dependent effect of (-)-a-pinene on antimicrobial resistance in C. jejuni, leading to either inhibition of antimicrobial efflux in lower concentrations while in higher concentrations leading to increased permeability of the cell membrane thereby promoting the influx of antimicrobials. Our results indicate that terpenes emitted by F. culmorum make the cell wall more permeable for VOCs to enter and to be taken up by the cell.

Volatiles may play a role as elicitors that activate gene clusters involved in secondary metabolites production. Likewise, antibiotic production triggered by volatiles mediated interactions was observed in Pseudomonas aeruginosa during co-culture with Enterobacter aerogenes and in P. fluorescens PfO-1 in response to volatiles produced by Collimonas pratensis (Garbeva et al., 2014, Venkataraman et al., 2014).

Moreover, in Chromobacterium violaceum and P. aeruginosa, several monoterpenes increased violacein and pyocyanin production, respectively (Ahmad et al., 2015). Here we observed an up-regulation of a terpene synthase gene encoding the synthesis of a terpene volatile compound. This compound 
was identified as sodorifen, an unusual volatile with extraordinary structure, where every carbon atom is substituted with either methyl or methylene group (Domik et al., 2016). However, little is known about the biosynthesis of sodorifen other than the study by Domik et al. (2016) that reports that a terpene synthase is indispensable for the synthesis of sodorifen and that it descends from the terpene metabolism. Similarly, knockout mutants of the methyl transferase found in the same operon showed no sodorifen production (Domik et al., 2016). We confirmed in our work that a terpene synthase and methyltransferase are essential for the biosynthesis of sodorifen by co-expressing SpSS and SpMT in E. coli. Furthermore, we demonstrated that methylation of FPP is necessary before the cyclisation by the terpene synthase. This was similarly demonstrated by Wang \& Cane (2008) for the biosynthesis of methylisoborneol, a methylated monoterpene with an earthy odor, from Streptomyces coelicolor.

The sodorifen synthase from Serratia is a multiproduct terpene synthase and sodorifen was produced together with a cluster of other terpene compounds. This is in agreement with the analysis of knockout mutants of either the terpene cyclase or the methyl transferase of the sodorifen operon which lacked the production of sodorifen including a number of other terpenoids (Domik et al., 2016). Sodorifen has been described to be produced by several Serratia species (Weise et al.). Even though S. plymuthica PRI-2C was previously reported as a non-producer of sodorifen, this work reveals that the compound is produced under certain conditions and in even higher amounts in response to fungal VOCs. Moreover, in a recent study that described the sodorifen cluster in S. plymuthica 4R×13 (Domik et al., 2016), the cluster was not detected in S. plymuthica PRI-2C when using antiSMASH. This is most likely due to the fact that at the time of the study the genome annotation of this strain was still in draft state and the described cluster could not be found. Comparison of the cluster with the complete genome of S. plymuthica PRI-2C shows a very high sequence similarity at the amino acid level of all four genes of the cluster to other Serratia strains including S. plymuthica $4 \mathrm{R} \times 13$.

This work indicates that terpenes may be important molecules in the communication between different microorganisms and hence, may serve as a lingua franca in fungal- bacteria interactions. 


\section{Acknowledgements}

This work is supported by The Netherlands Organization for Scientific Research (NWO) VIDI personal grant to Paolina Garbeva (864.11.015). This is publication 6238 of the NIOO-KNAW.

\section{Author contributions}

R.S. designed and conducted transcriptomics, proteomics and metabolomics experiments, analyzed the data and wrote the manuscript. V.D.J. contributed to the analysis of the transcriptomics data and provided genome assembly and annotation; D.Z. assisted in the proteomics design, conduction of the experiment, data analysis and in writing the manuscript; C.W. assisted in the proteomics experiment; J.B. assisted in the proteomics data analysis; K.C. performed heterologous expression experiments; J.B. contributed to the design of the heterologous expression experiments and edited the manuscript; W.V.I and F.S. performed RNA-Sequencing. W.D.B. edited the manuscript; K.R. contributed to the design of the proteomics experiments and edited the manuscript; P.G. assisted in the design of all experiments and edited the manuscript. All authors read and approved the manuscript.

\section{Competing interest}

The authors declare no competing financial interests. 


\title{
Supplementary Materials
}

\author{
qPCR \\ RNA sequencing
}

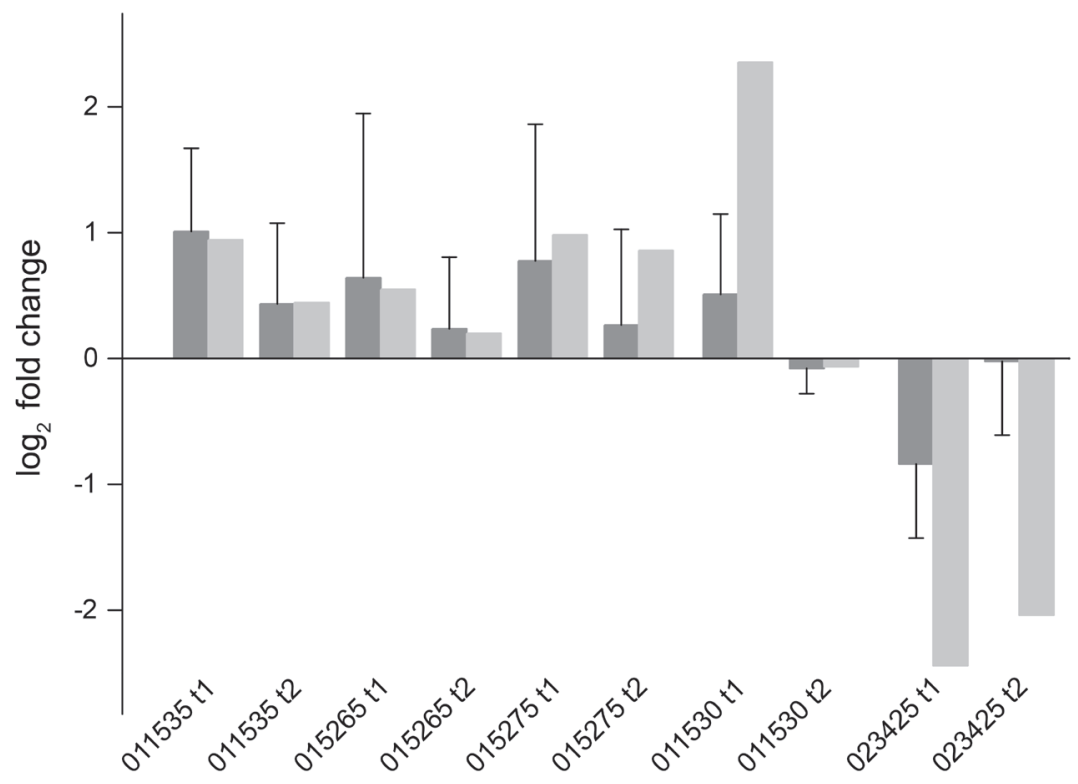

Fig. S1 / qPCR validation of the RNA-seq data at two time points (t1 and t2); 011535 (Putative terpene synthase), 015265 (Flagellin), 015275 (Flagellar protein FliS), 011530 (Arabinose operon regulatory protein), 023425 (Isocitrate lyase). $\log _{2}$ fold changes determined from the relative Ct values of the five genes were compared to those detected by RNA-seq. All Ct values were normalized to two housekeeping genes (rpoB and gyrB). Positive values correspond to higher expression and negative values to lower expression in S. plymuthica PRI-2C samples. 
Table S1 / signal transduction systems in S. plymuthica PRI-2C

\begin{tabular}{|c|c|c|c|}
\hline type & input functions & output functions & \\
\hline \multirow[t]{14}{*}{$1 \mathrm{cp}$} & Cofactor binding, Small-molecule binding & DNA-binding & 1 \\
\hline & Enzymatic & DNA-binding & 17 \\
\hline & Enzymatic, Small-molecule binding & Hydrolase & 1 \\
\hline & Small-molecule binding & Di-guanylate cyclase & 2 \\
\hline & Small-molecule binding & DNA-binding & 181 \\
\hline & Small-molecule binding & RNA-binding & 1 \\
\hline & Unknown & Di-guanylate cyclase & 1 \\
\hline & & Adenylate cyclase & 1 \\
\hline & & Di-guanylate cyclase & 13 \\
\hline & & DNA-binding & 201 \\
\hline & & Hydrolase & 2 \\
\hline & & Other & 5 \\
\hline & & Protein kinase & 1 \\
\hline & & RNA-binding & 1 \\
\hline Total & & & 428 \\
\hline \multirow[t]{5}{*}{$2 \mathrm{cp}$} & Small-molecule binding & Protein kinase & 1 \\
\hline & Small-molecule binding & & 8 \\
\hline & Unknown & & 1 \\
\hline & & DNA-binding & 31 \\
\hline & & & 28 \\
\hline Total & & & 69 \\
\hline ecf & & ECF & 2 \\
\hline Total & & & 2 \\
\hline chemotaxis & Small-molecule binding & & 1 \\
\hline & & & 7 \\
\hline Total & & & 8 \\
\hline other & Small-molecule binding & & 5 \\
\hline Total & & & 5 \\
\hline
\end{tabular}

$1 \mathrm{cp}=$ Oe-component system, $2 \mathrm{cp}=$ Two-component system , Ecf = Extracytoplasmic function 


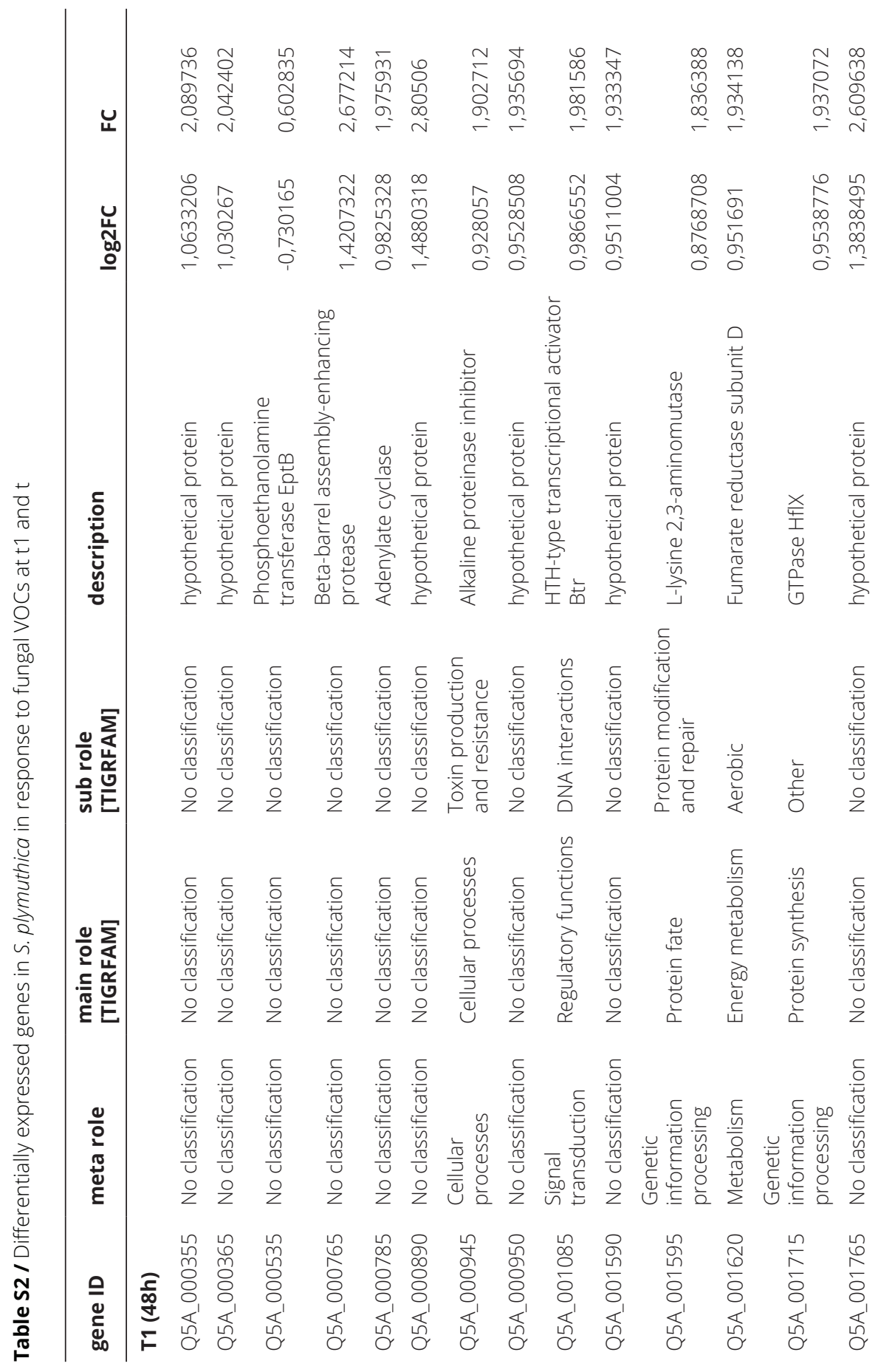




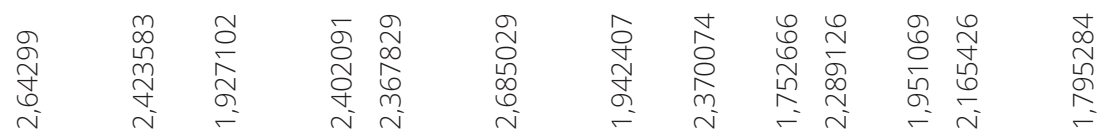

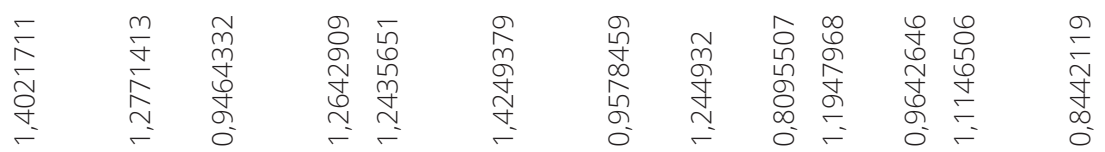

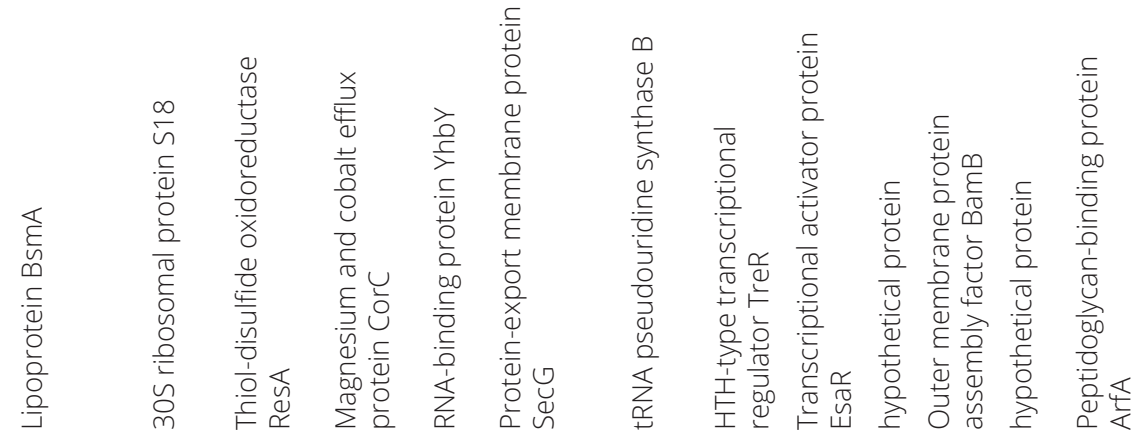

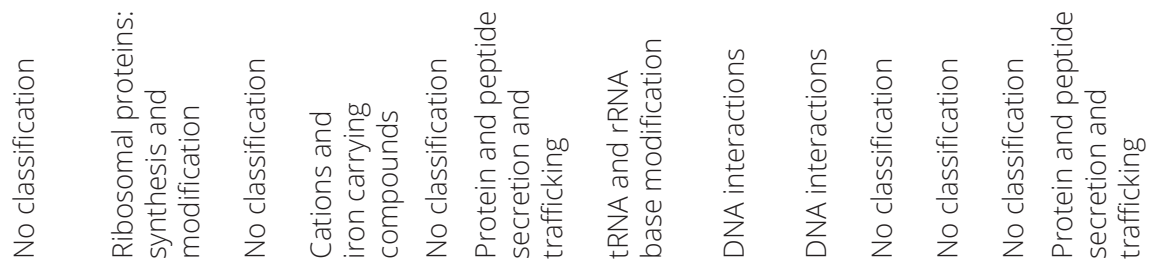

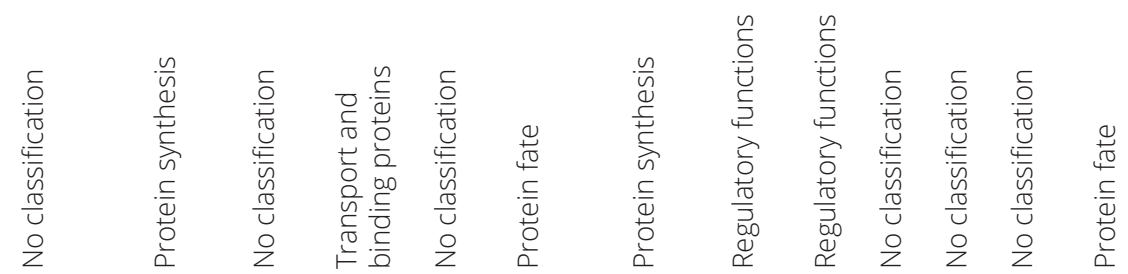

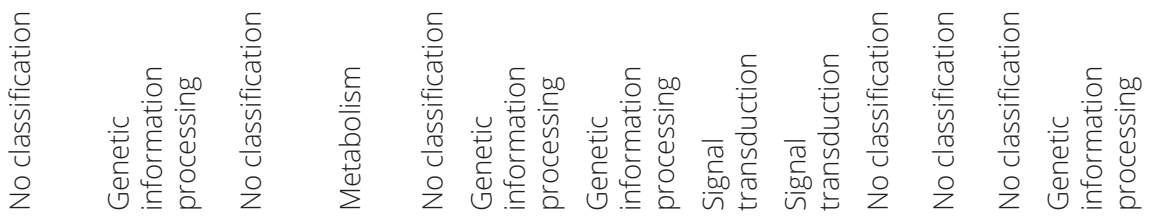

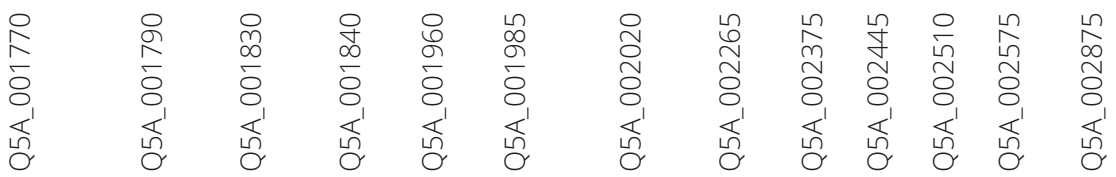




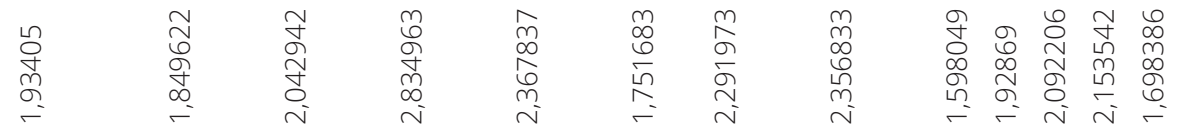

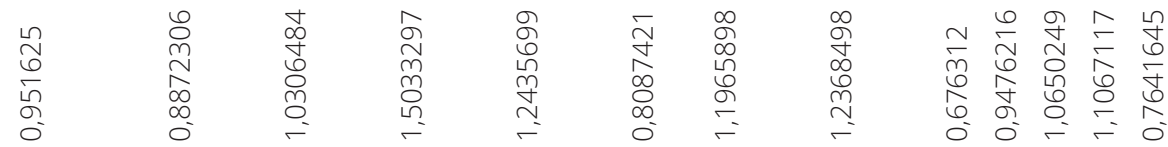

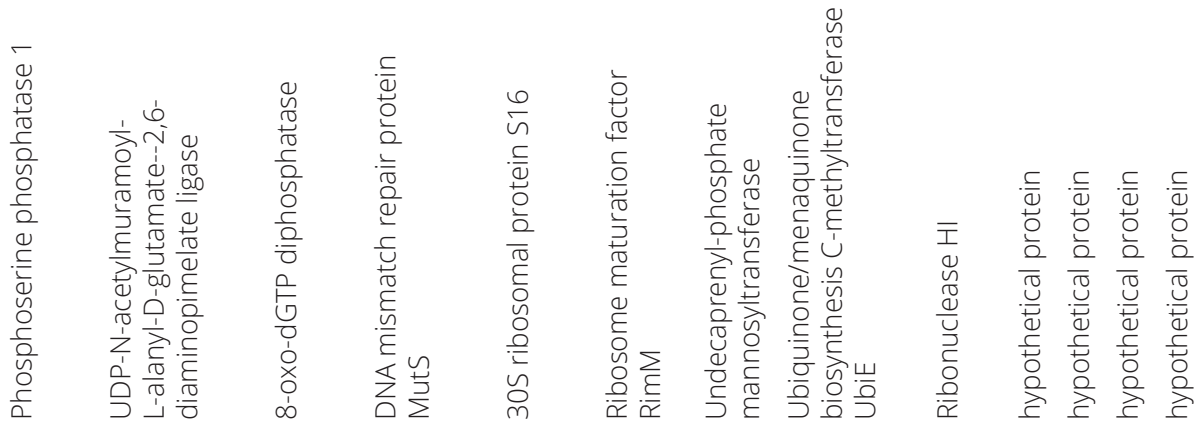

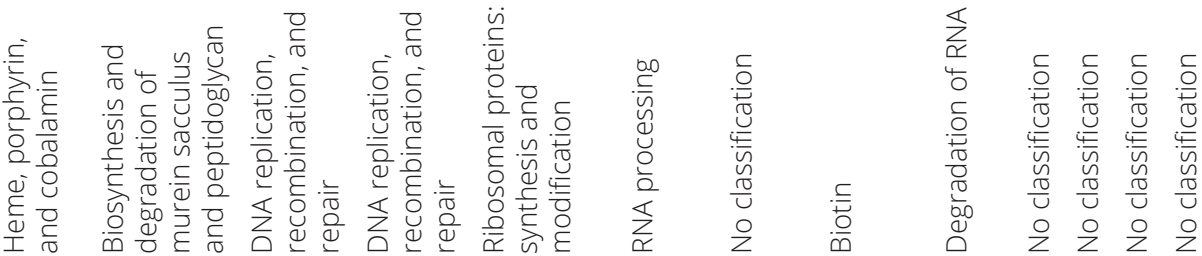

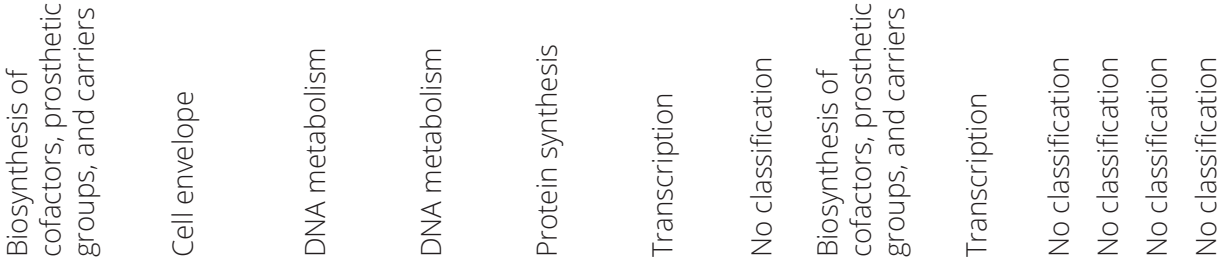

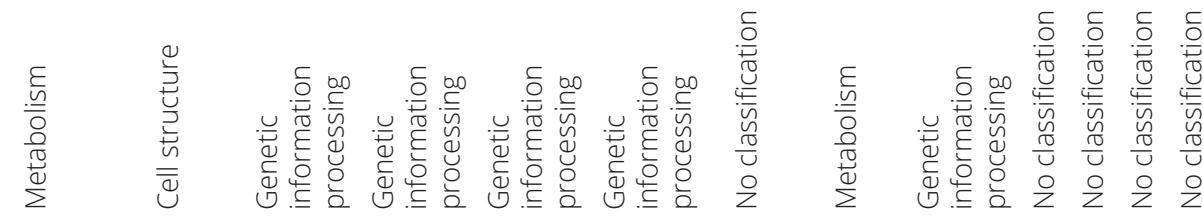

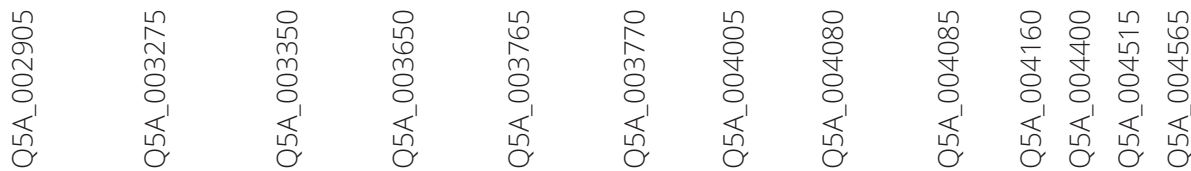




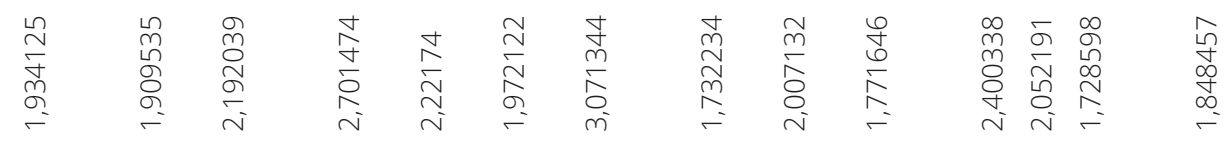

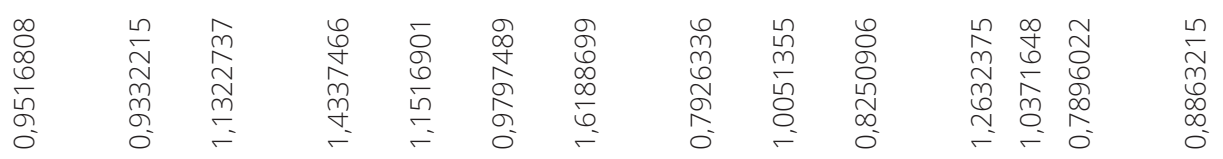

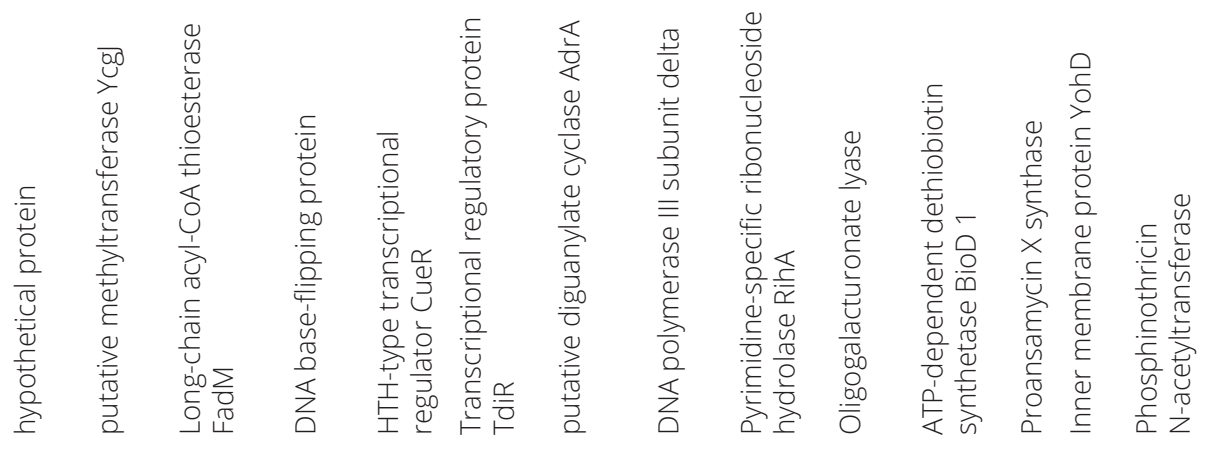

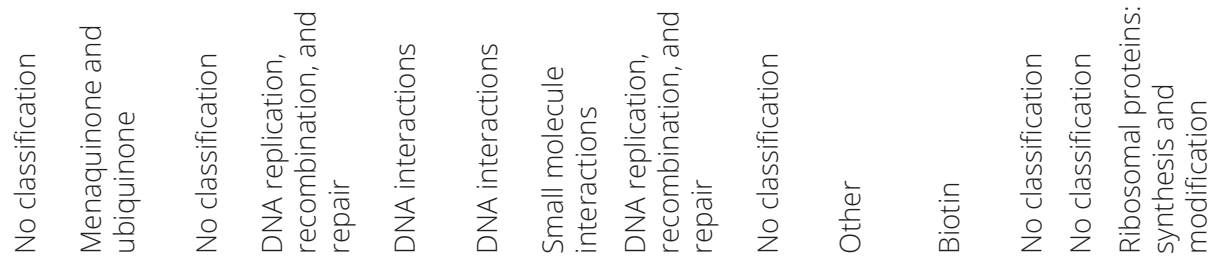

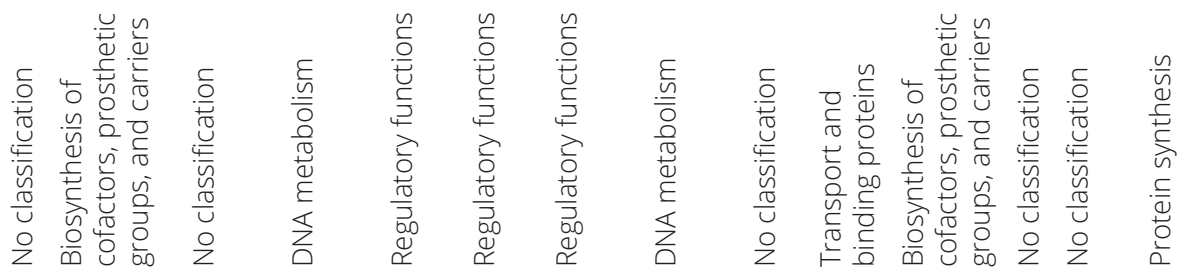

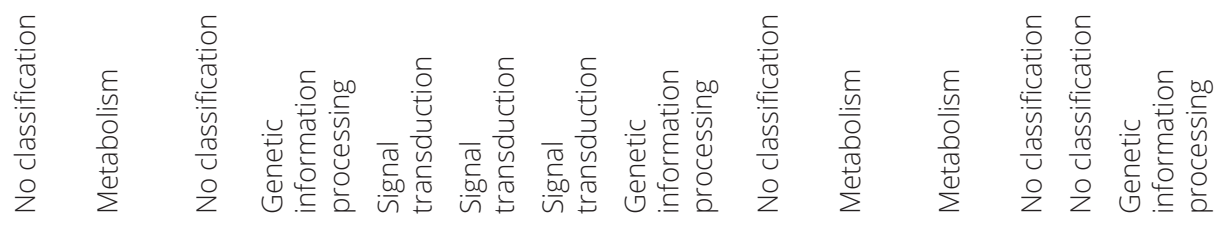

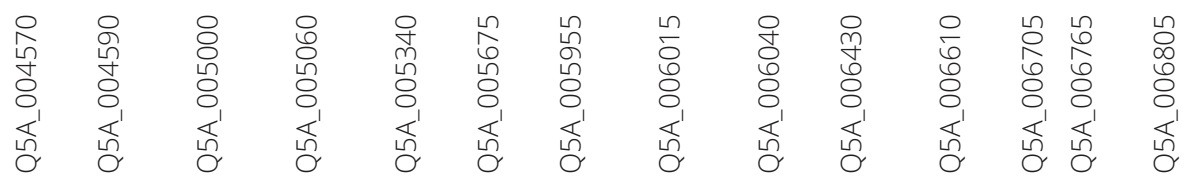




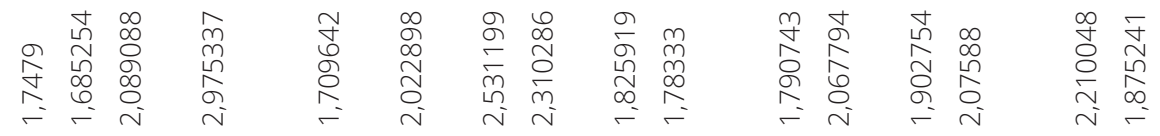

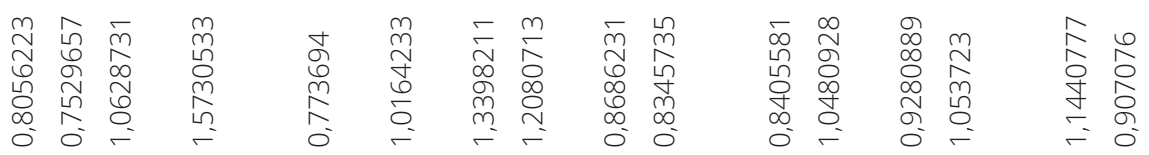

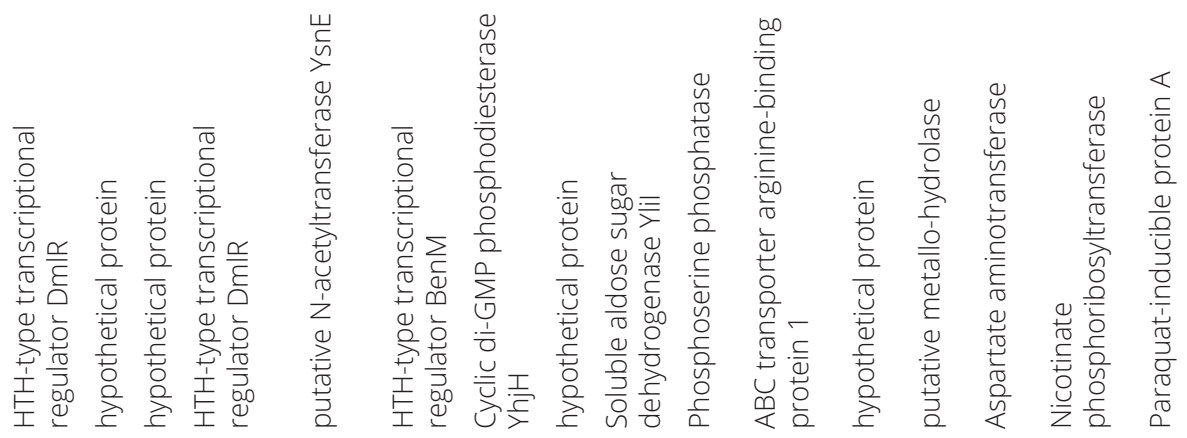

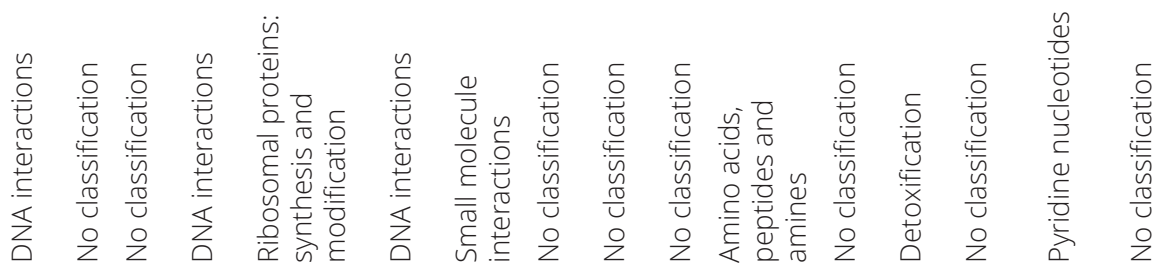

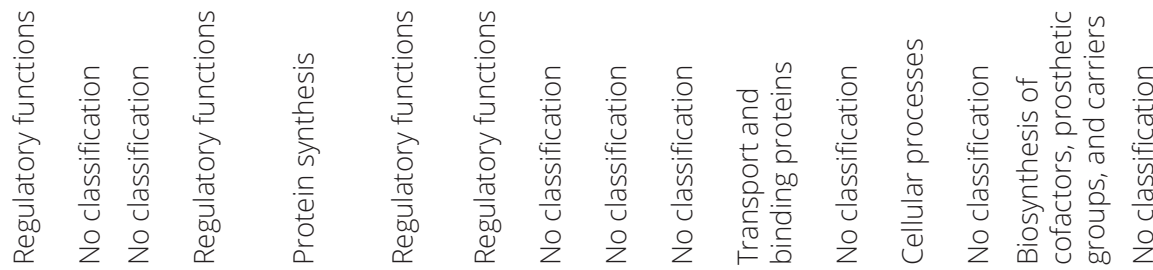

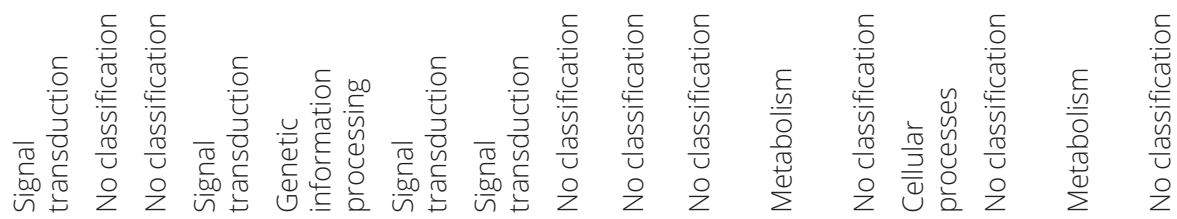

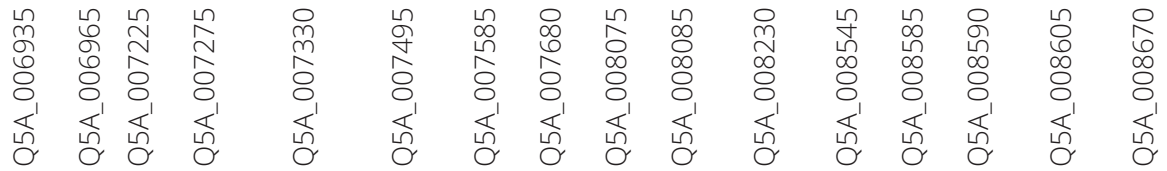




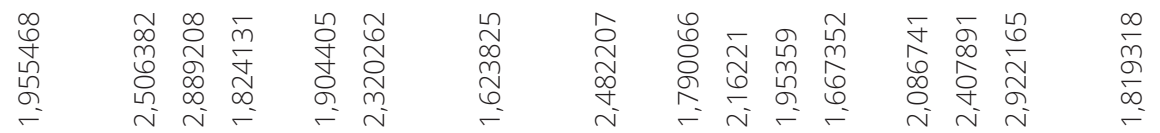

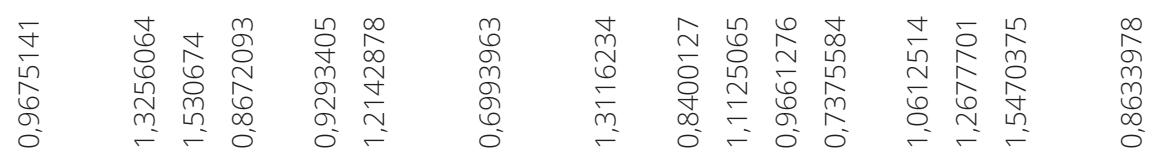

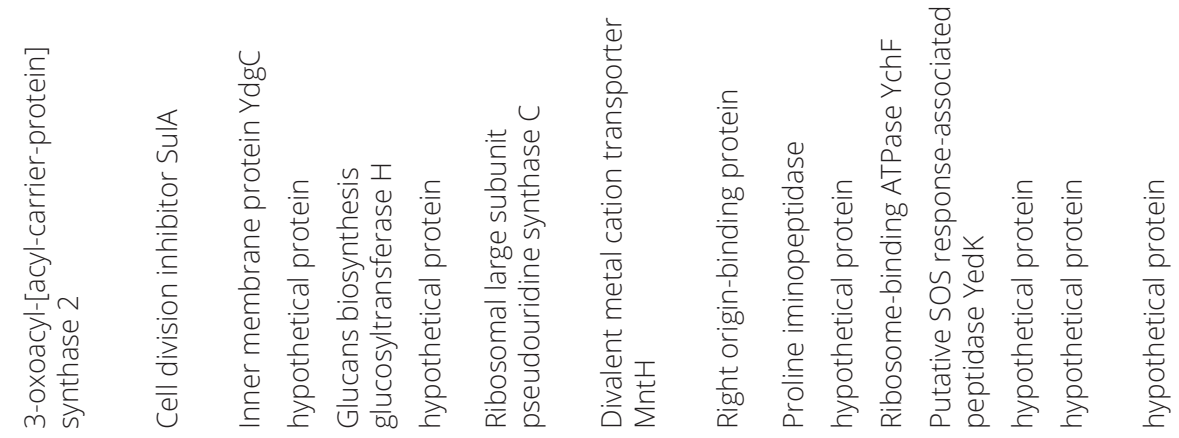

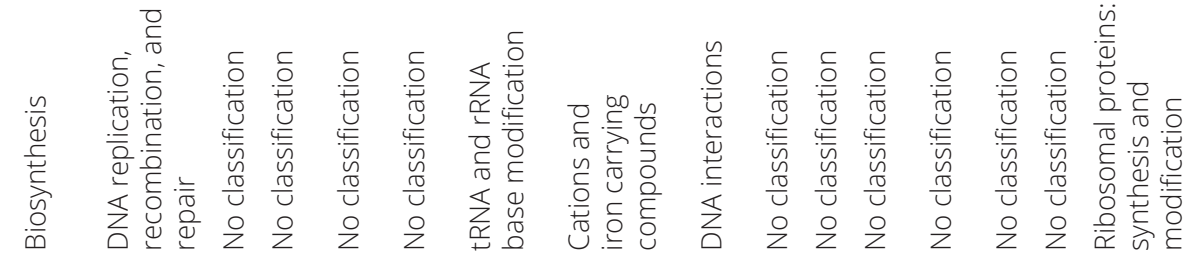

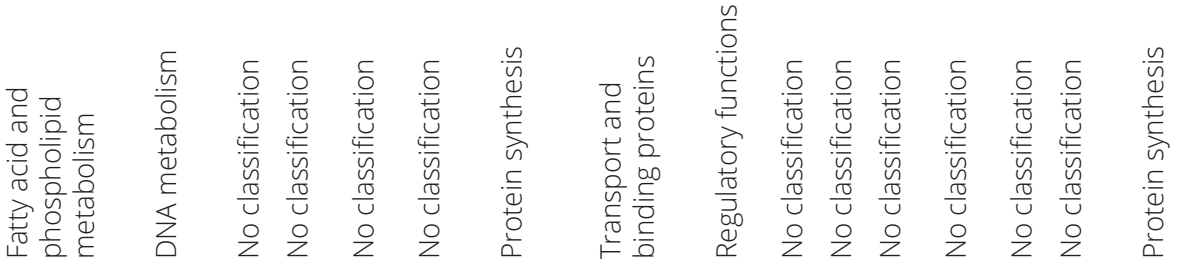

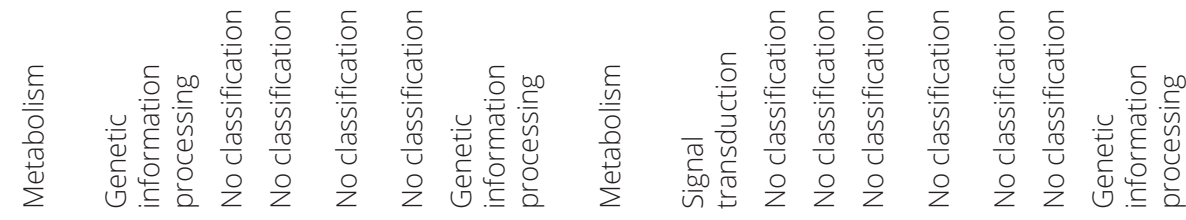

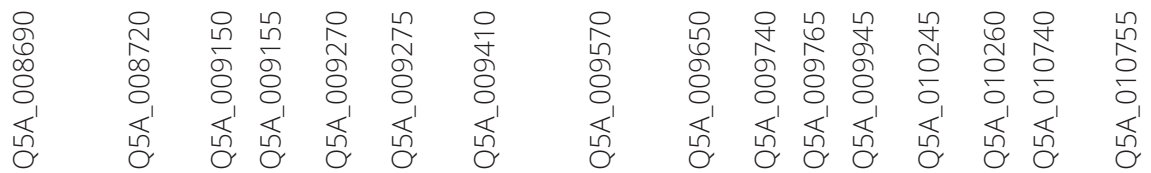




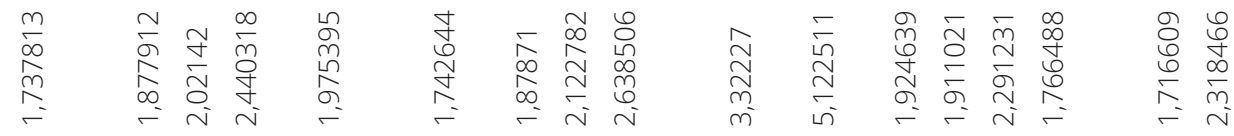

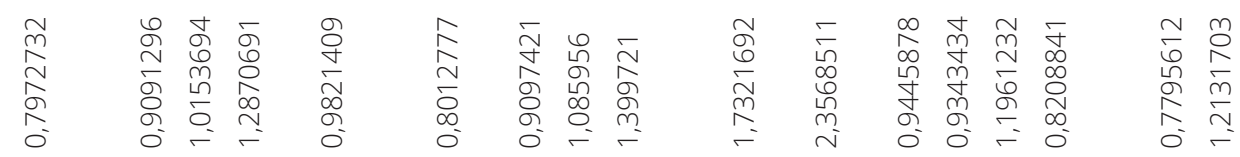

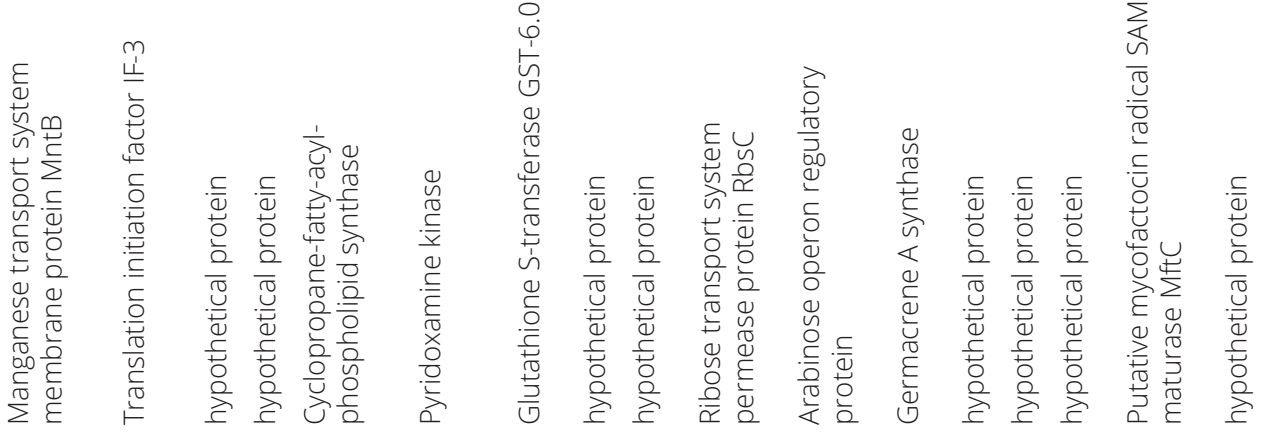

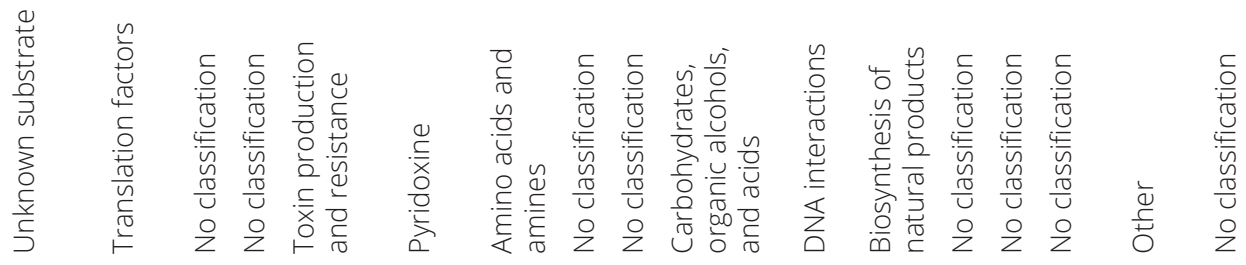

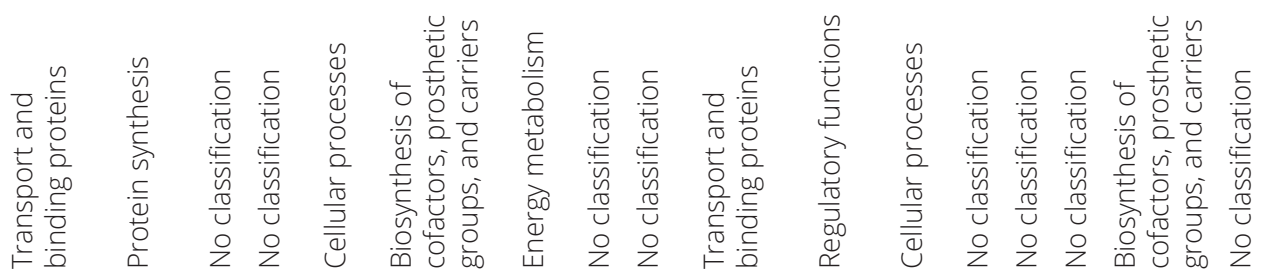

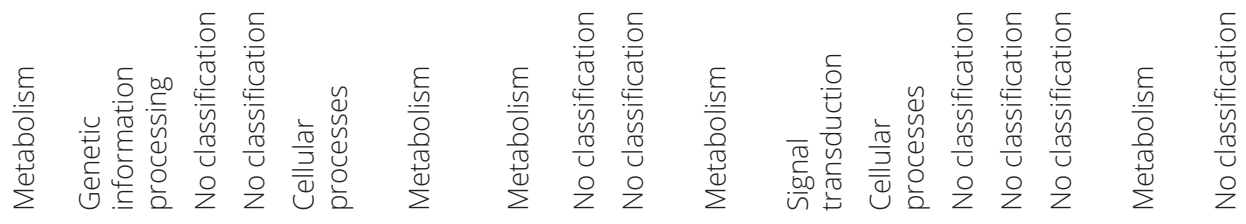

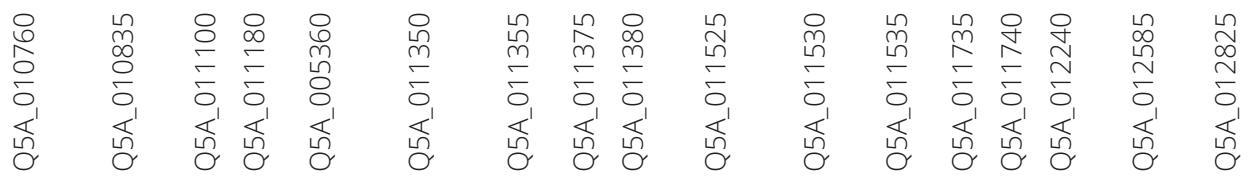




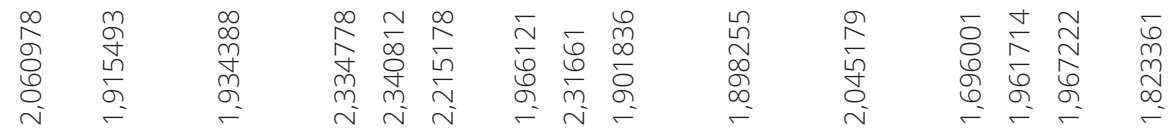

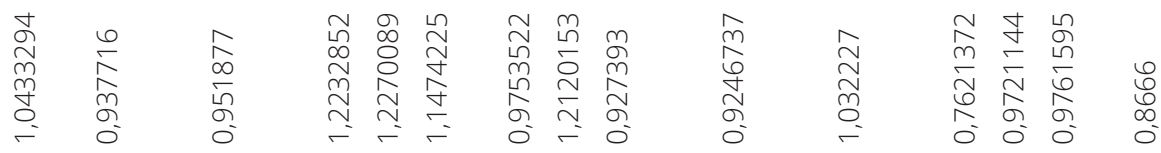

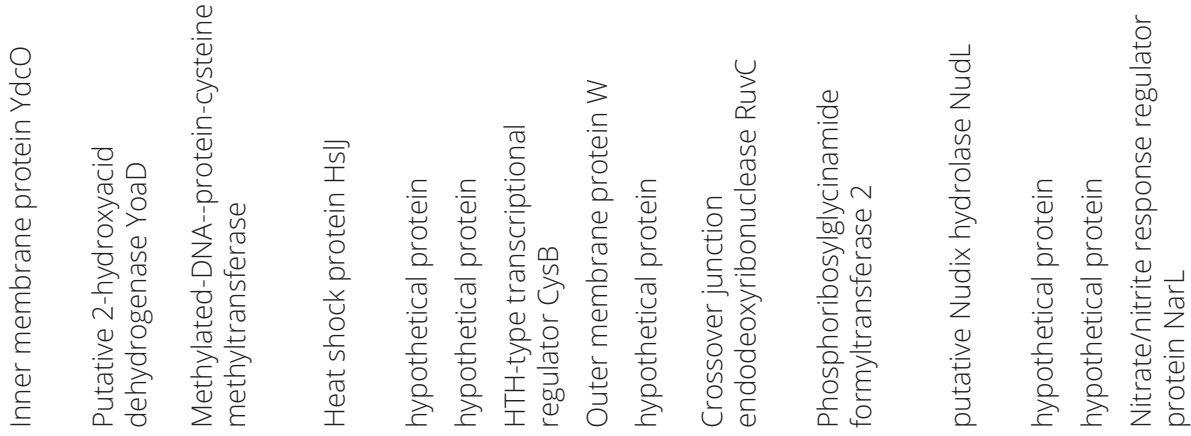

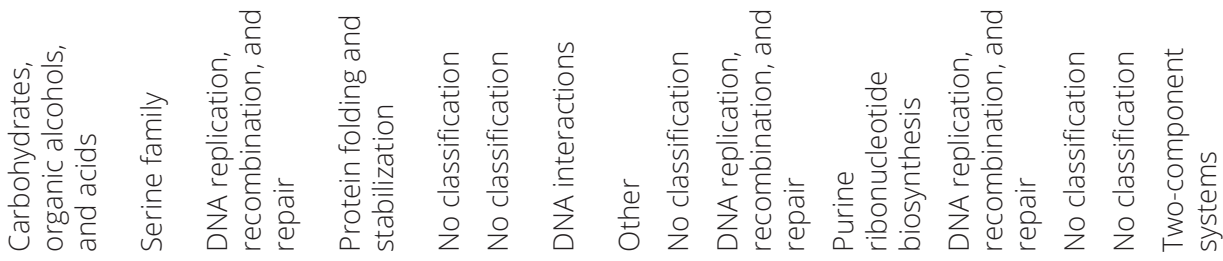

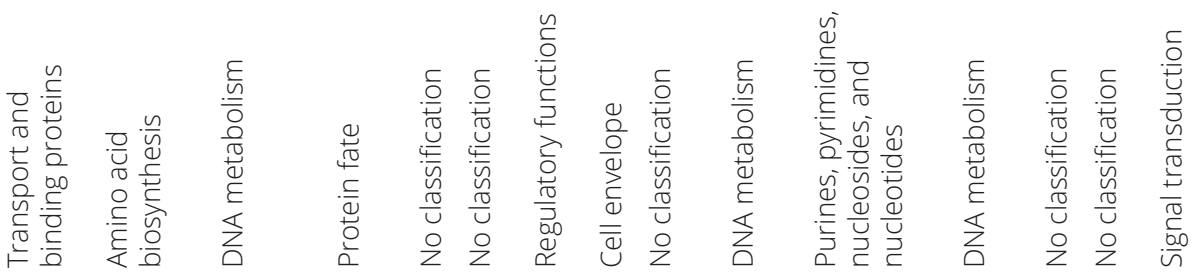

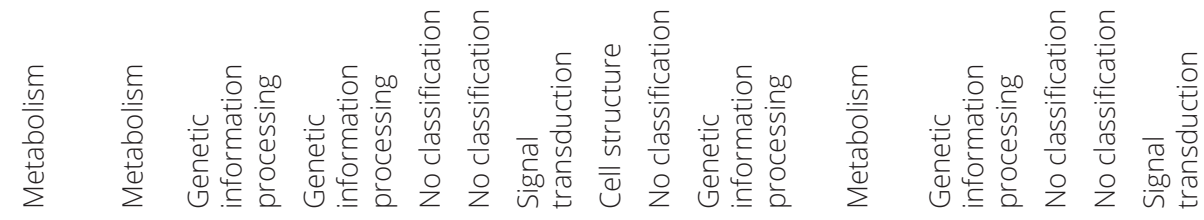

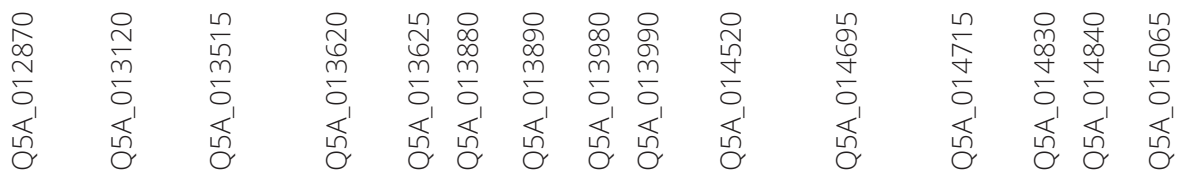




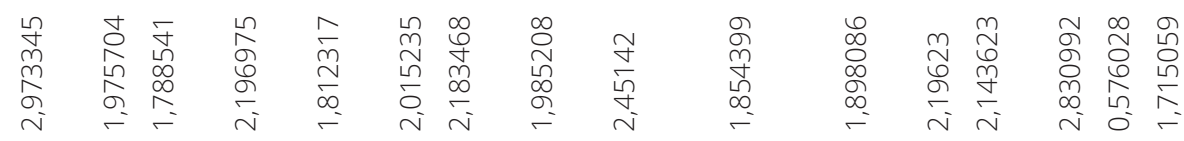

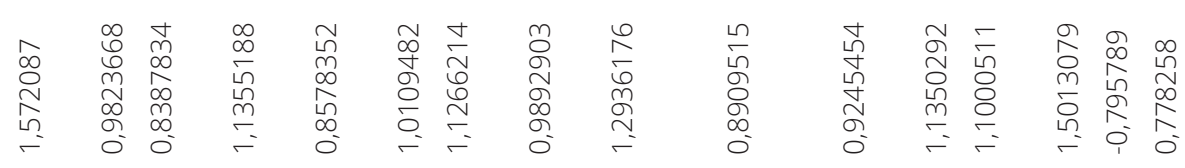

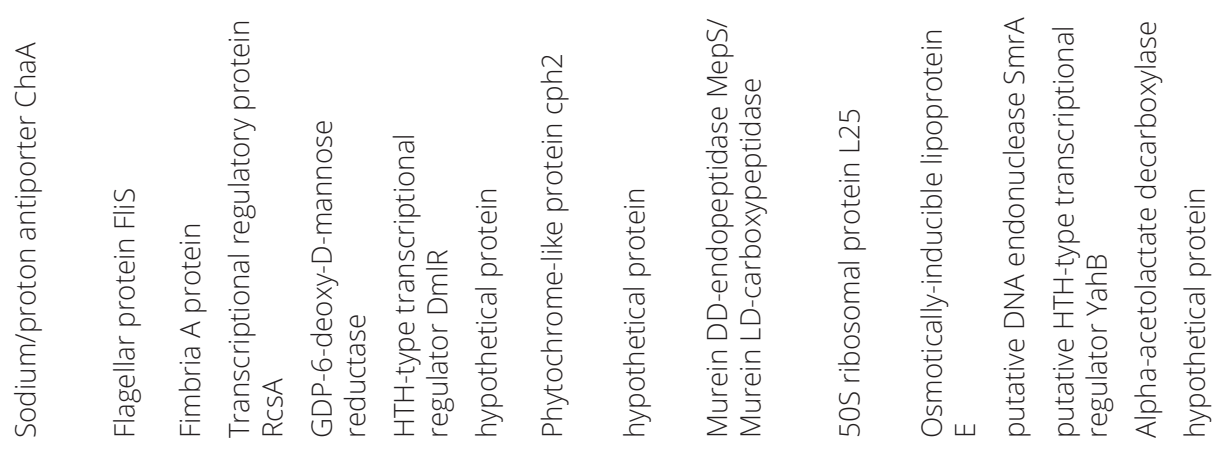

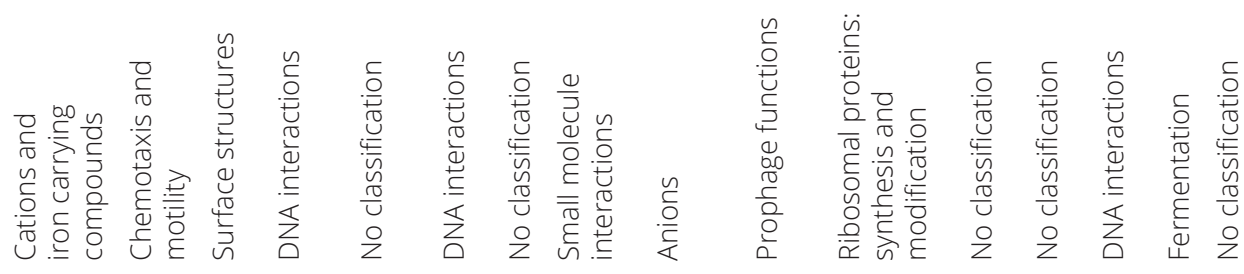

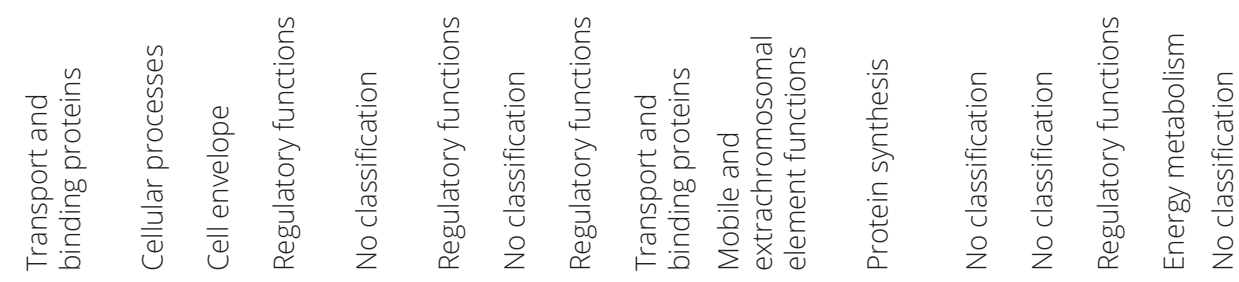

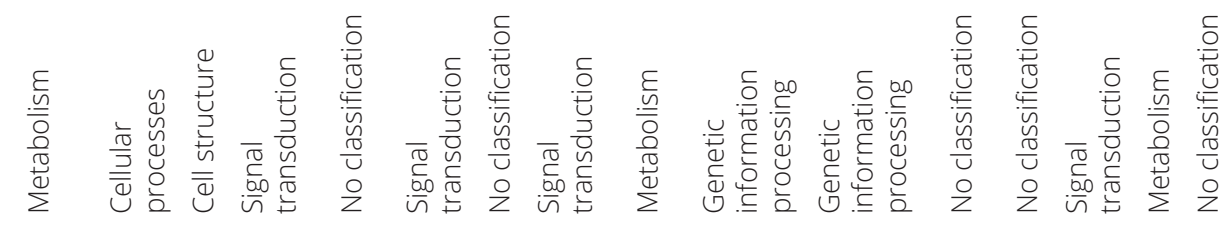

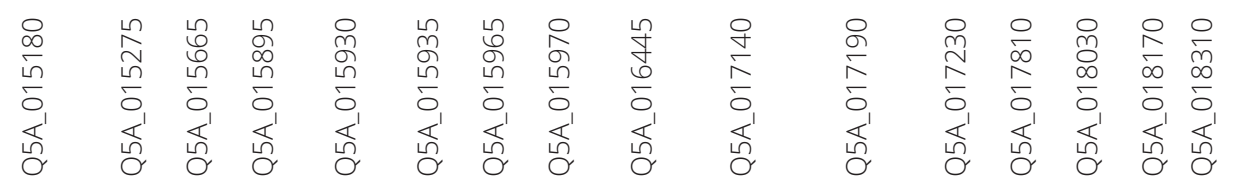




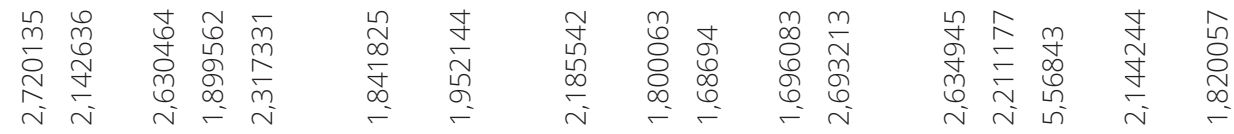

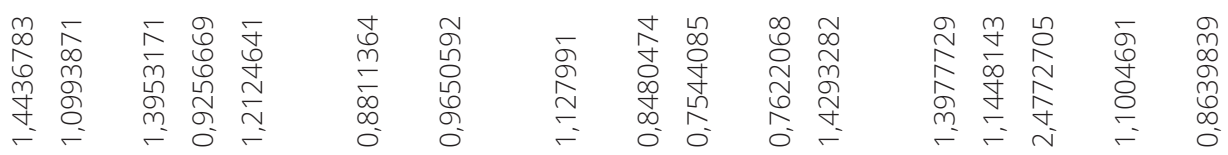

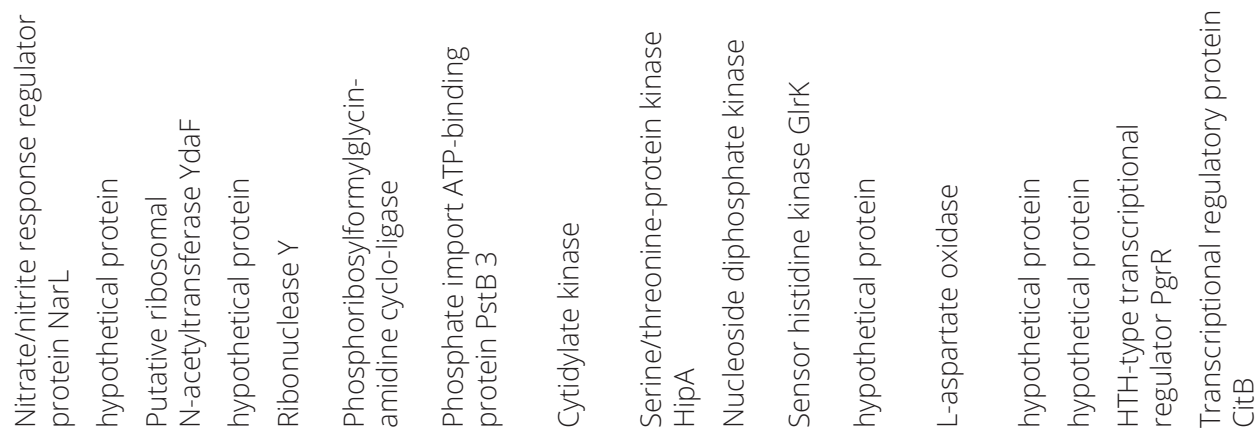

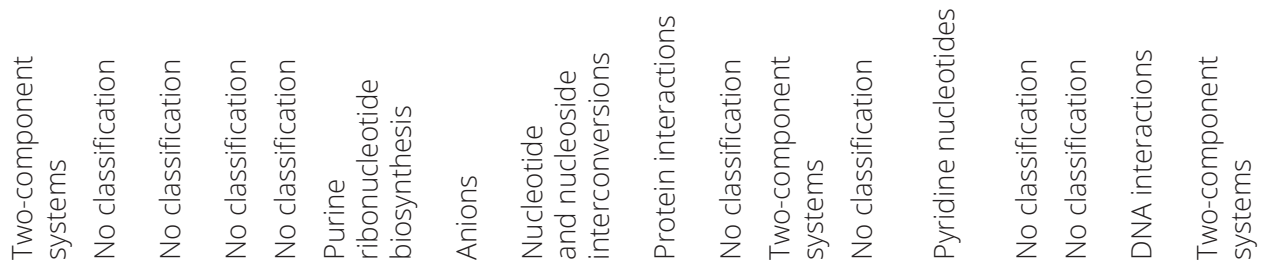

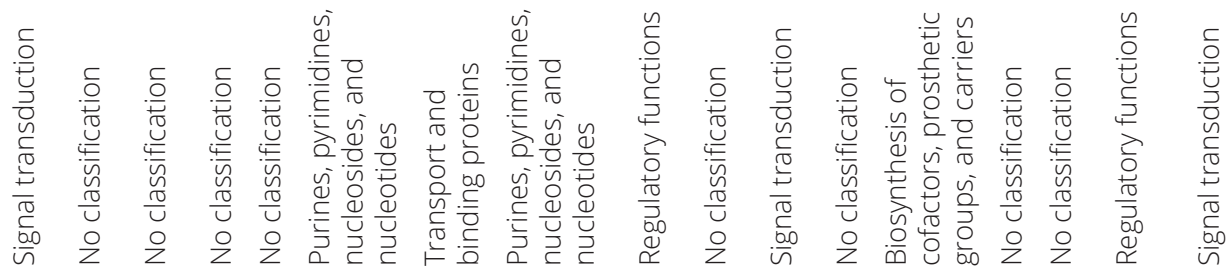

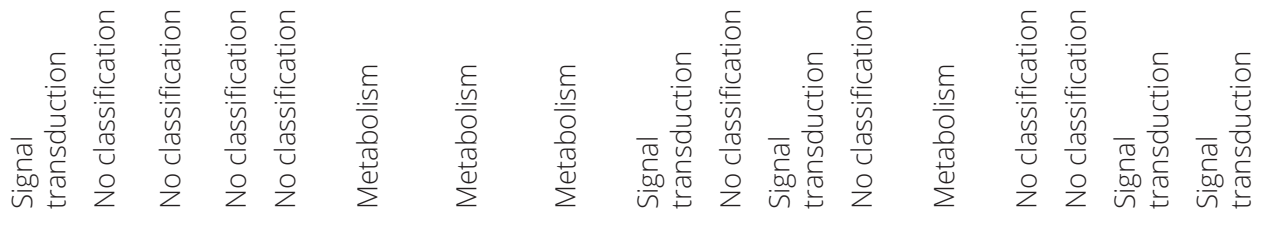

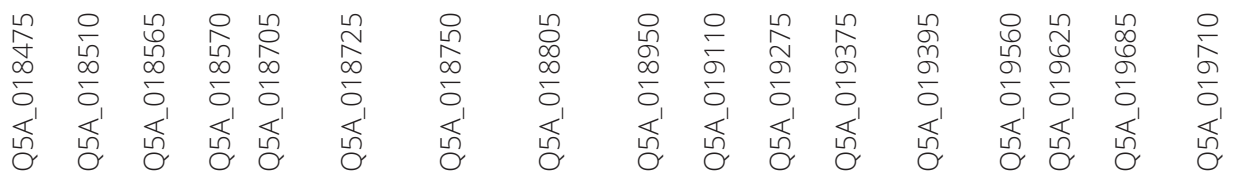




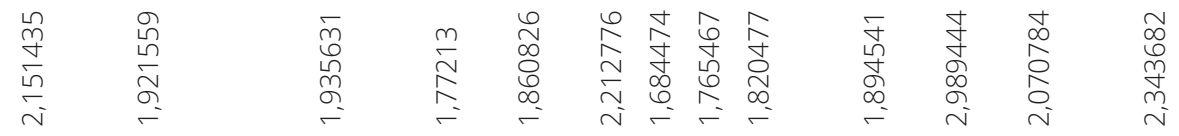

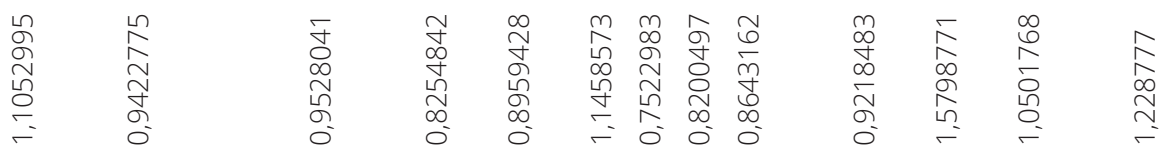

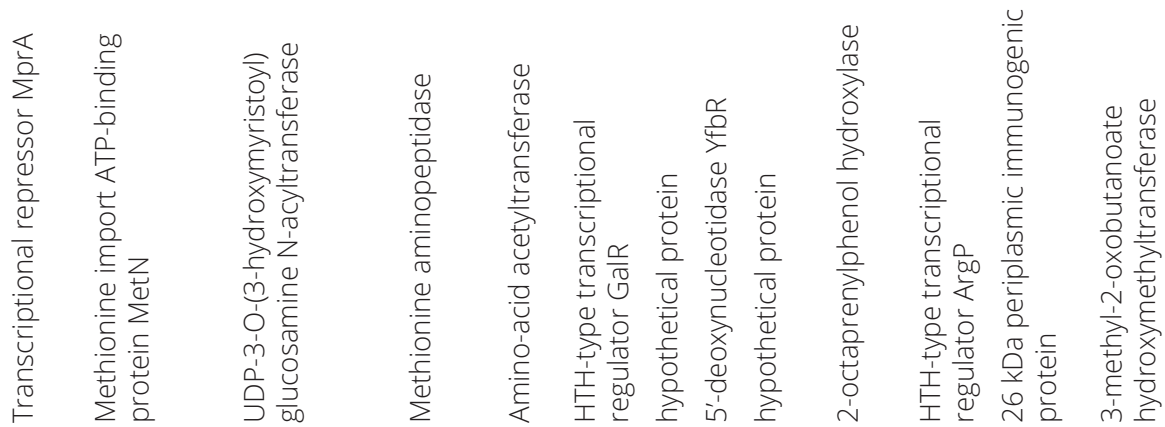

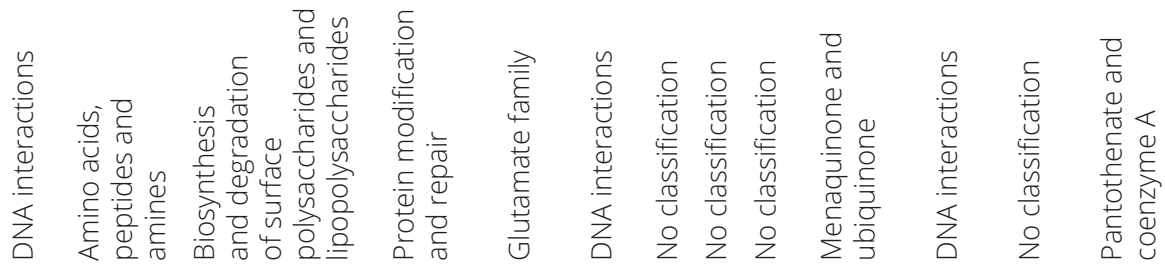

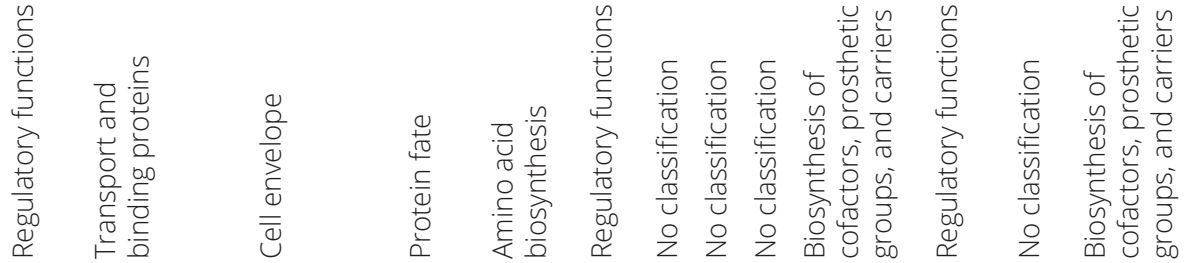

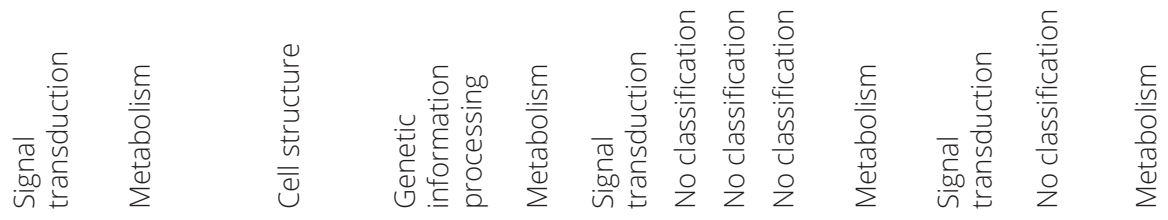

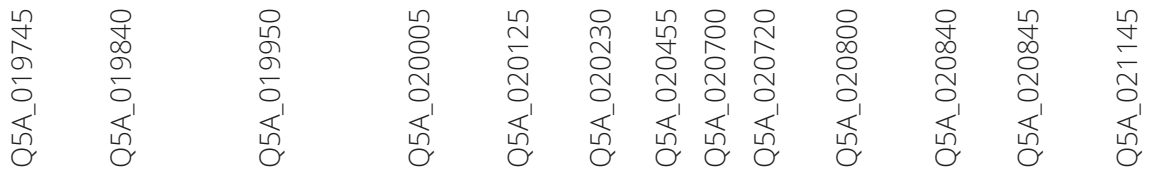




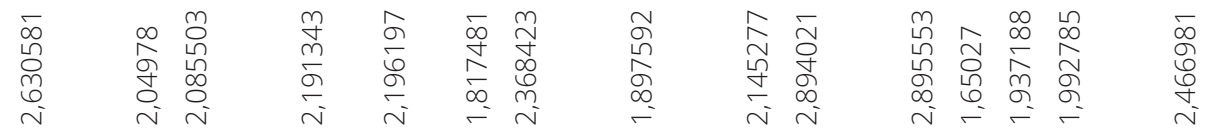

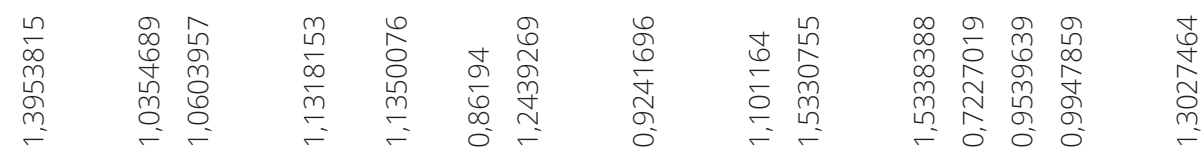

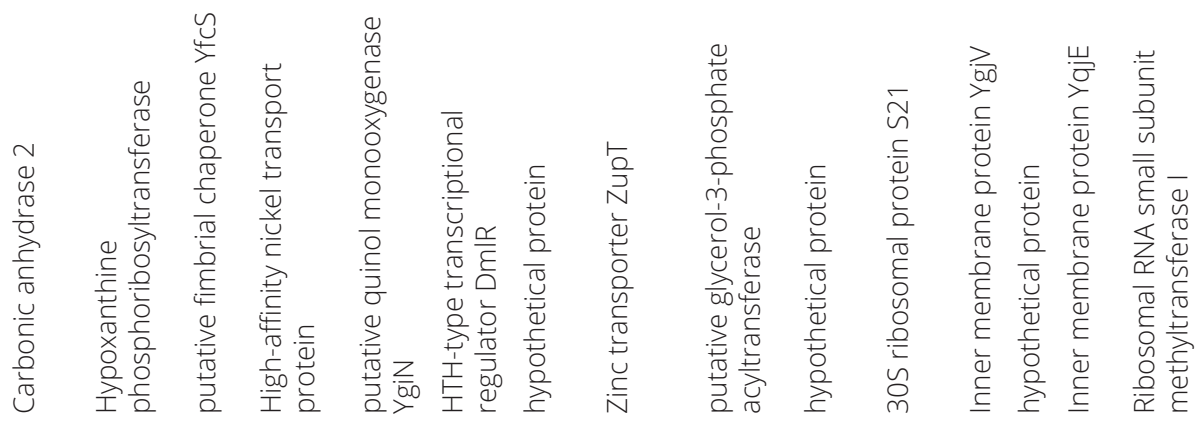

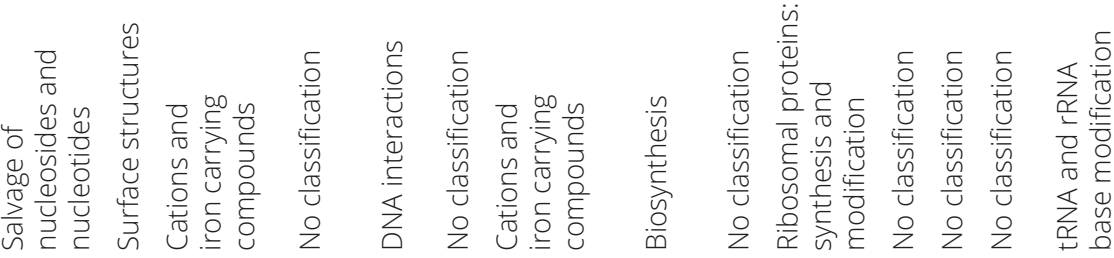

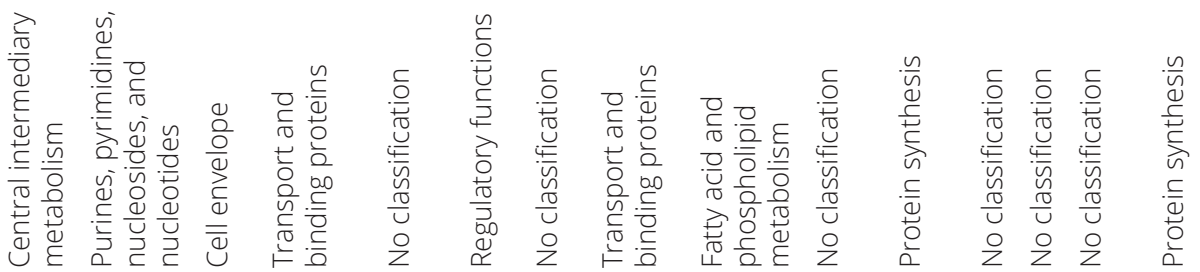

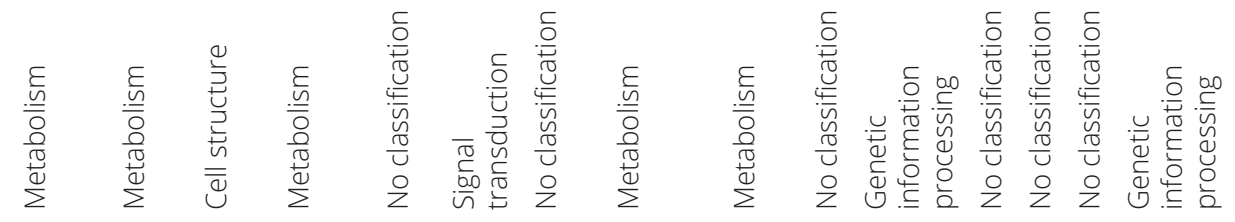

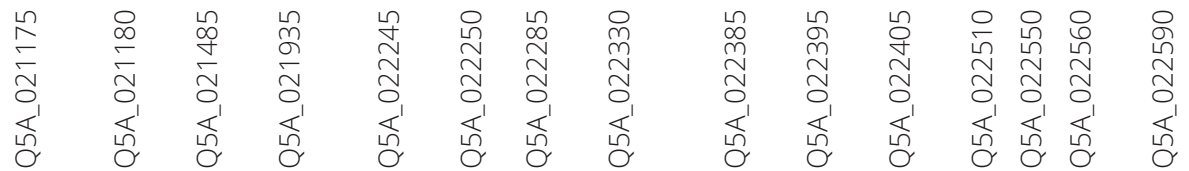




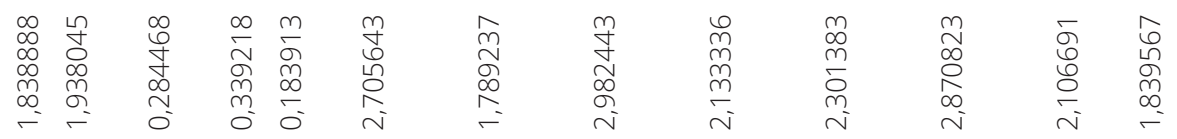

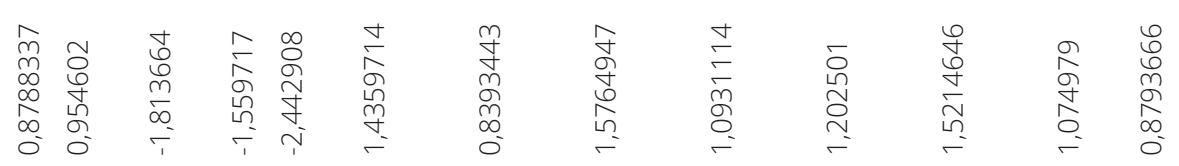

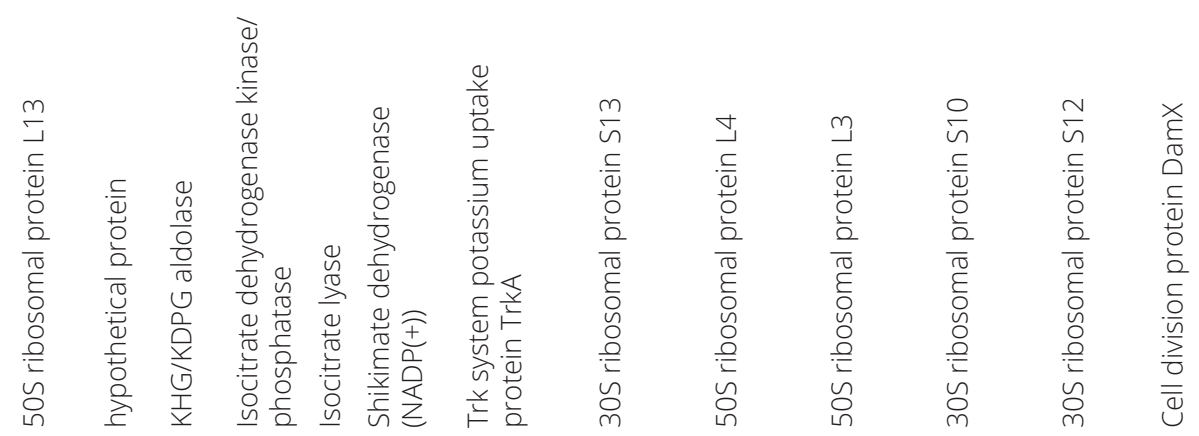

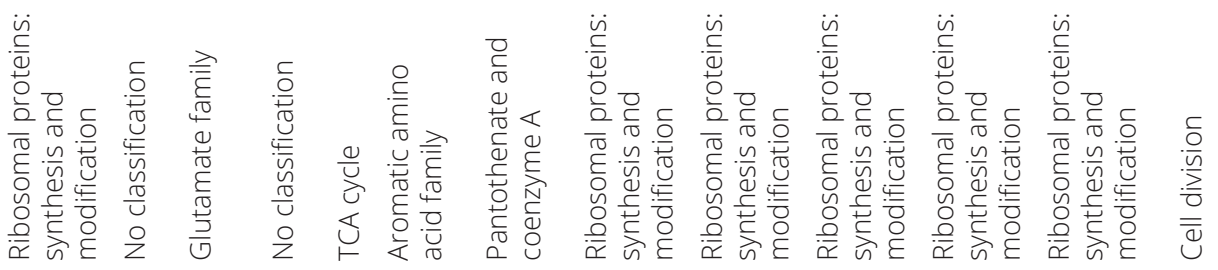

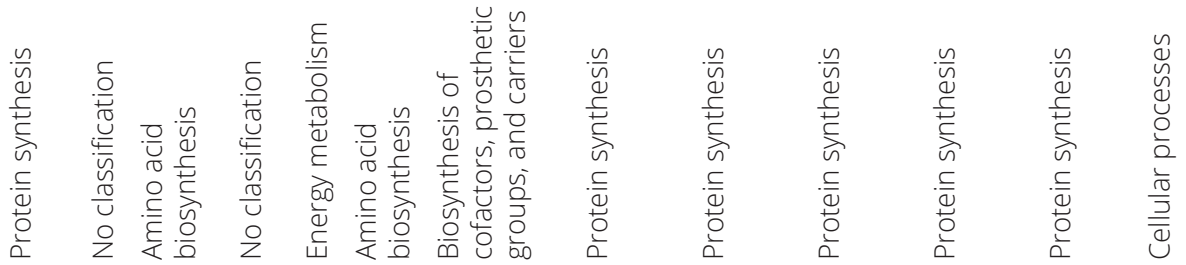

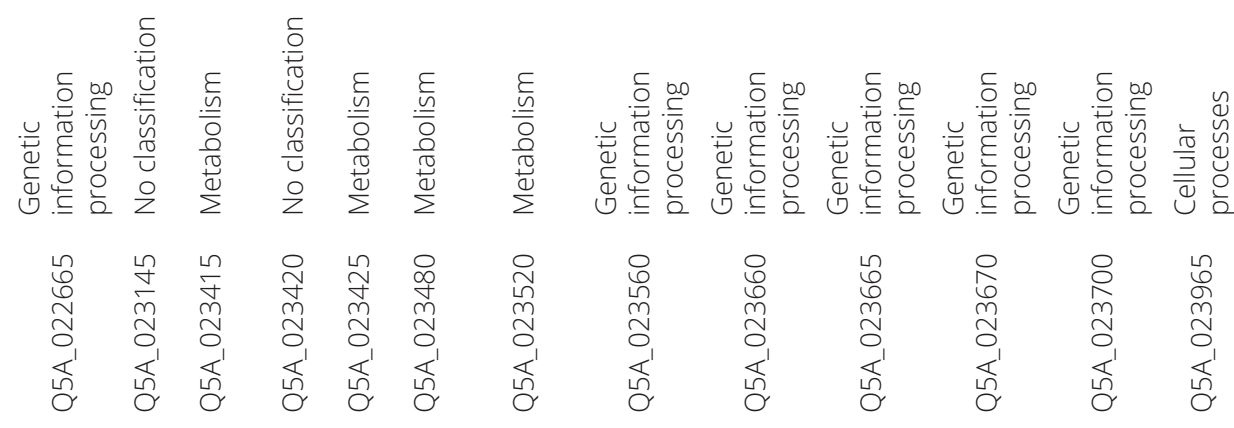




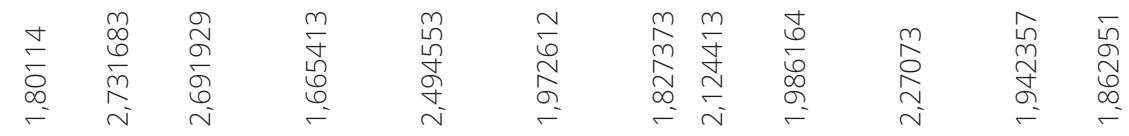

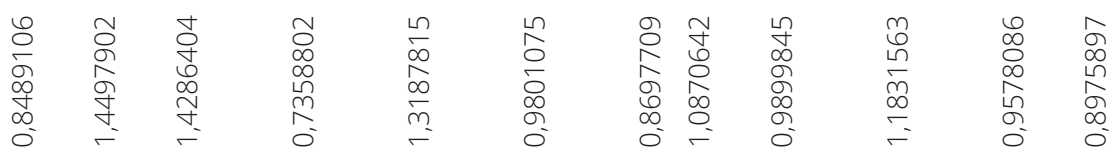

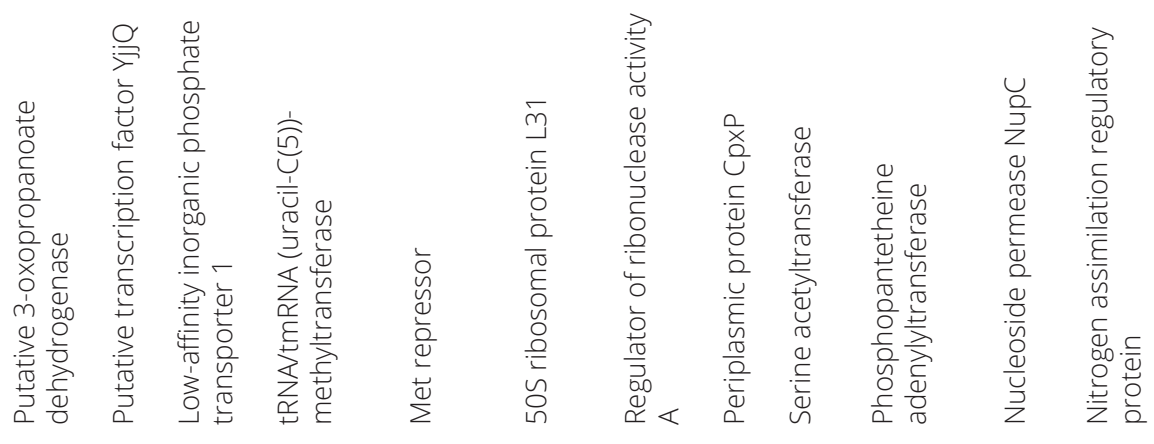

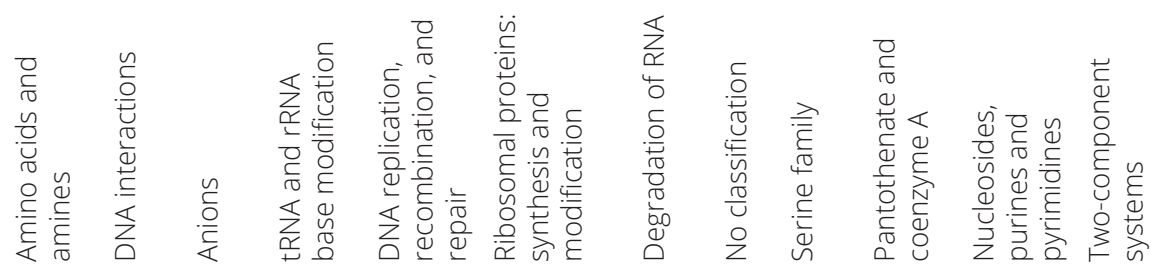

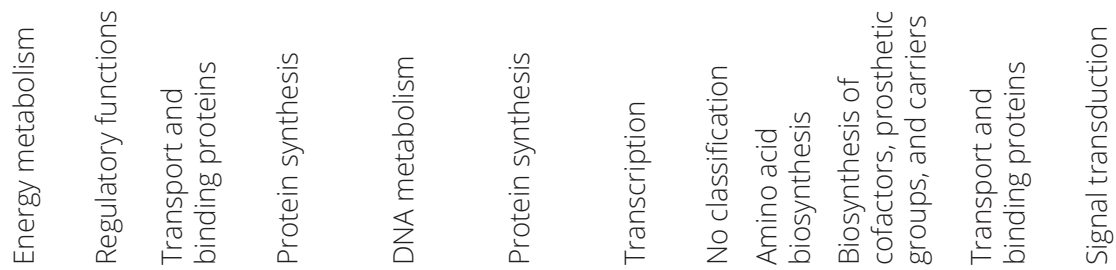

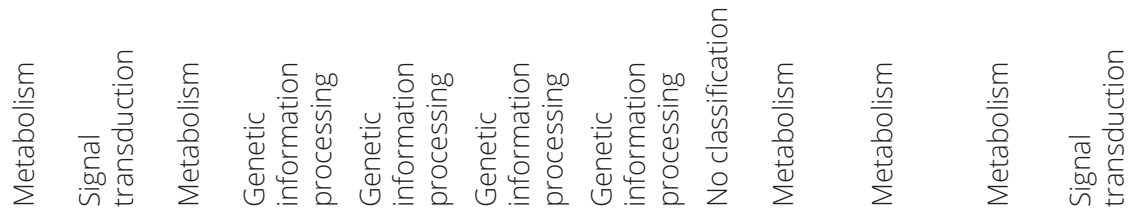

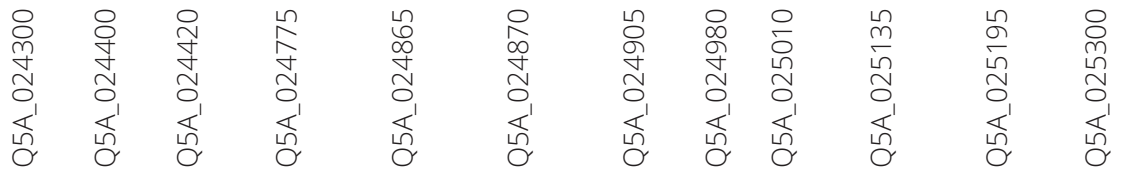




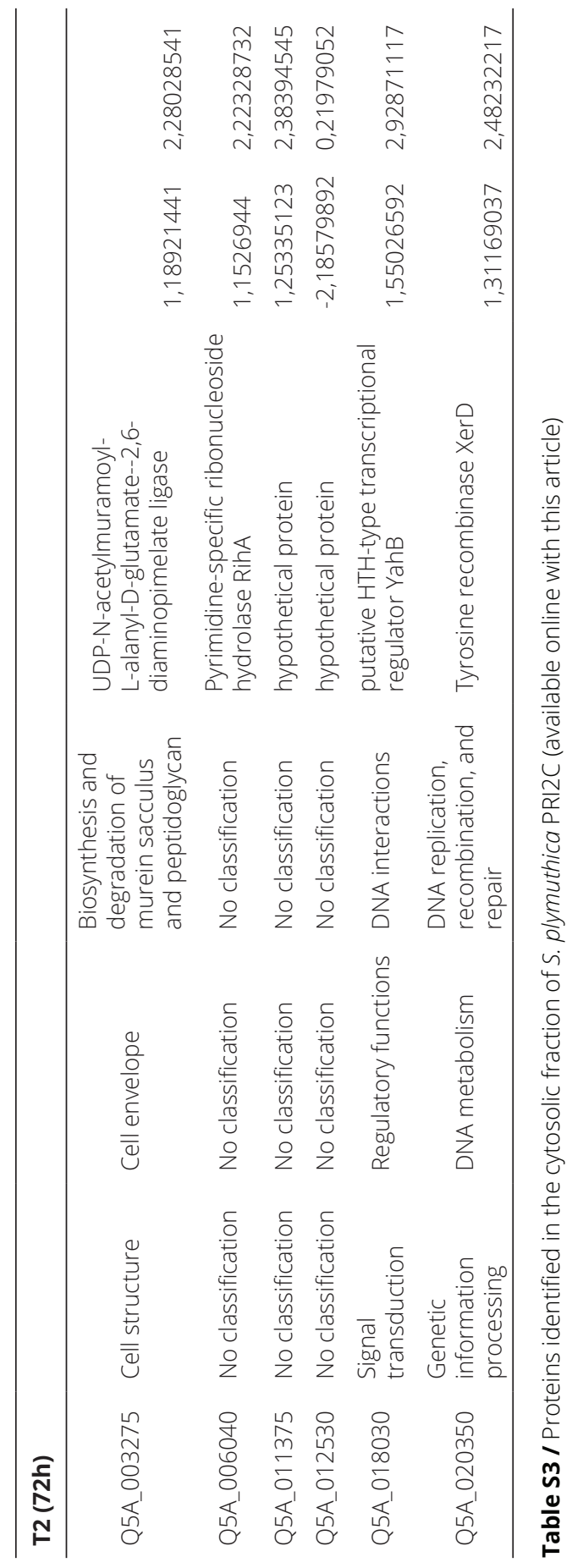




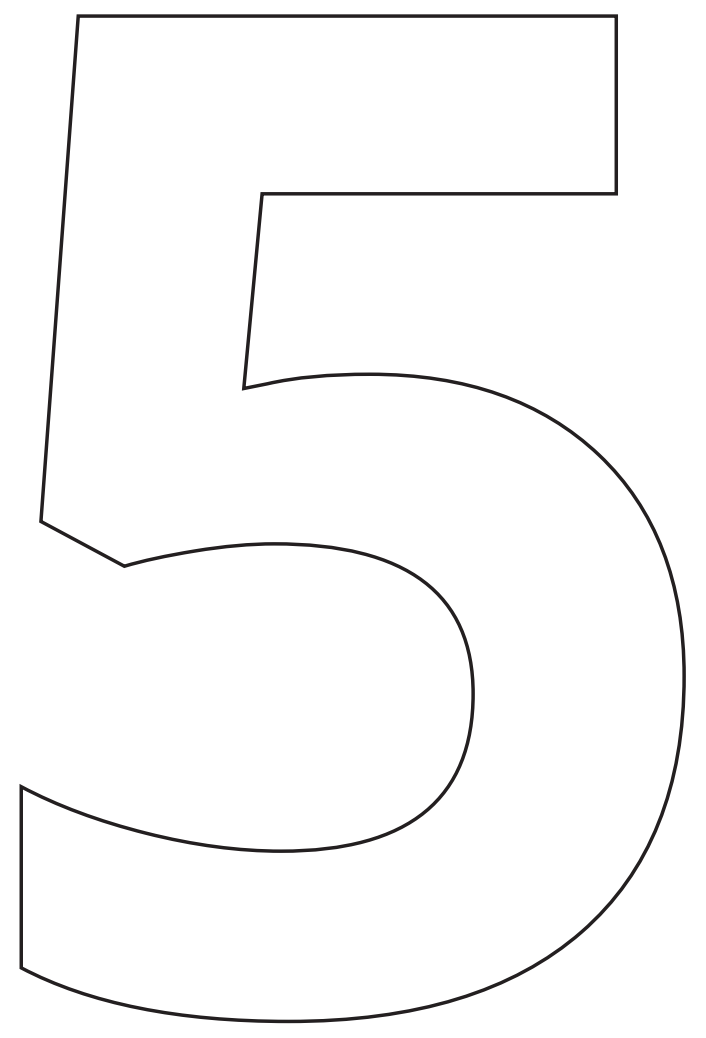

\section{Exploring the genomic traits of fungus-feeding bacterial genus Collimonas}

Chunxu Song, Ruth Schmidt, Victor de Jager, Dorota Krzyzanowska, Esmer Jongedijk, Katarina Cankar, Jules Beekwilder, Anouk van Veen, Wietse de Boer, Johannes A. van Veen and Paolina Garbeva

BMC Genomics (2015) 



\section{Abstract}

\section{Background}

Collimonas is a genus belonging to the class of Betaproteobacteria and consists mostly of soil bacteria with the ability to exploit living fungi as food source (mycophagy). Collimonas strains differ in a range of activities, including swimming motility, quorum sensing, extracellular protease activity, siderophore production, and antimicrobial activities.

\section{Results}

In order to reveal ecological traits possibly related to Collimonas lifestyle and secondary metabolites production, we performed a comparative genomics analysis based on whole-genome sequencing of six strains representing 3 recognized species. The analysis revealed that the core genome represents $49.8 \%$ to $59.6 \%$ of the genomes of the six individual strains. These include genes coding for extracellular enzymes (chitinase, peptidase, phospholipase), iron acquisition and type II secretion systems. In the variable genome, differences were found in genes coding for secondary metabolites (e.g. tripropeptin A and volatile terpenes), several unknown orphan polyketide synthase-nonribosomal peptide synthetase (PKSNRPS), nonribosomal peptide synthetase (NRPS) gene clusters, a new lipopeptide and type III and type VI secretion systems. Potential roles of the latter genes in the interaction with other organisms were investigated. Mutation of a gene involved in tripropeptin A biosynthesis strongly reduced the antibacterial activity against Staphylococcus aureus, while disruption of a gene involved in the biosynthesis of the new lipopeptide had a large effect on the antifungal/oomycetal activities.

\section{Conclusions}

Overall our results indicated that Collimonas genomes harbor many genes encoding for novel enzymes and secondary metabolites (including terpenes) important for interactions with other organisms and revealed genomic plasticity, which reflect the behavior, antimicrobial activity and lifestyles of Collimonas spp.

Keywords: comparative genomics, Collimonas, secondary metabolites, terpenes 


\section{Background}

The genus Collimonas comprises soil bacteria with the ability to grow at the expense of living fungal hyphae under nutrient-limited conditions (De Boer et al., 1998, de Boer et al., 2004, Leveau et al., 2010). Since the first description of Collimonas, more mycophagous bacteria have been detected (Rudnick, 2015), but Collimonas species are still highly interesting in view of the interactions between bacteria and fungi in soil and the associated ecosystem functions including suppression of pathogens and the production of novel bioactive compounds.

Collimonas belongs to the family Oxalobacteraceae, class Betaproteobacteria. The first Collimonas isolates were obtained within the framework of a project searching for a naturally occurring biocontrol agent of fungi pathogenic to marram grass (Ammophilia arenaria) and were determined as being dominant among the cultivable chitinolytic bacteria in the acidic Dutch dune soils (De Boer et al., 1998). Three species have been described so far: C. fungivorans, C. pratensis and C. arenae (Hoppener-Ogawa et al., 2008). All three species display the ability to feed on fungi (mycophagy), to degrade chitin and to dissolve minerals (weathering) (Leveau et al., 2010). However, Collimonas strains differ in important ecological traits such as colony morphology, the ability to oxidize various carbon sources, in their antibacterial, antifungal and antioomycetal activities (Mela et al., 2011, Mela et al., 2012). A comparative genomic approach would help to reveal the genetic basis of these ecological differences. Applying a comparative genomic hybridization approach (Mela et al., 2012) showed that a gene cluster involved in the production of an antifungal polyyne was only found in the genome of a few Collimonas strains. The study of Mela et al (2012) was biased in the sense that the hybridization assay used allowed only to screen for absence/presence of genes in other strains as compared to C. fungivorans strain Ter 331, the only strain for which the complete genome was sequenced at that time. To reveal the real plasticity of Collimonas strains more genome sequences are needed. This will demonstrate constant and variable genetic elements, and hence determine the adaptations of Collimonas species and traits important for inter-specific microbial interactions in the soil. To date, only two genome sequences of Collimonas are publicly available (Mela et al., 2012, Wu et al., 2015). Here we report on full genome sequences of five Collimonas strains across the three recognized species and performed comparative genome analysis including the recently published C. fungivorans Ter331 genome (Mela et 
al., 2012). Gene clusters with potential relevance for interactions of the Collimonas species with fungi and other microorganisms were further investigated by gene knock-out mutations and/or enzymatic characterization.

\section{Results and discussion}

\section{Genomic features}

A genome sequence analysis was performed for strains Ter6, Ter91, Ter291, Ter10 and Ter282, using a combined strategy of Illumina Hiseq and PacBio sequencing. A summary of the general genomic features (size, GC content, predicted number of coding sequences, and number of rRNAs) of each Collimonas strain is presented in Table 1. Considerable variation in genome size and differences in plasmid content was observed. The six genomes vary in size by approximately one megabase (ranging from 4.7-5.7 Mb) with the number of coding sequences (CDSs) ranging from 4436-5424, indicating substantial strain-to-strain variation. The genomes of C. fungivorans and C. pratensis are larger and have higher GC content than the two strains of C. arenae. Only strain C. fungivorans Ter331 has a plasmid, described in detail by Mela et al (2008). Despite the absence of a plasmid, C. pratensis Ter91 has the largest genome size (5.7 kb) and highest number of encoding genes (5424), which is likely due to the large number of horizontally acquired genes as indicated by the number of genomic islands (see below). 
Table 1 / Collimonas strains and their genomic features

\begin{tabular}{|c|c|c|c|c|c|c|}
\hline \multirow[t]{2}{*}{ Feature } & \multicolumn{2}{|c|}{ Collimonas fungivorans } & \multicolumn{2}{|c|}{ Collimonas pratensis } & \multicolumn{2}{|c|}{ Collimonas arena } \\
\hline & Ter331 & Ter6 & Ter91 & Ter291 & Ter10 & Ter282 \\
\hline Source & \multicolumn{6}{|c|}{ Inner coastal dune soil in Terschelling, the Netherlands } \\
\hline $\begin{array}{l}\text { Chromosome size } \\
(\mathrm{Mb})\end{array}$ & 5.2 & 5.6 & 5.7 & 5.6 & 4.7 & 4.7 \\
\hline Plasmid size & $40 \mathrm{~kb}$ & NA & NA & NA & NA & NA \\
\hline $\mathrm{G}+\mathrm{C} \%$ & $59.6 \%$ & $59 \%$ & $58.8 \%$ & $59 \%$ & $56.8 \%$ & $56.8 \%$ \\
\hline $\begin{array}{l}\text { Protein-encoding } \\
\text { sequences (CDSs) }\end{array}$ & 4910 & 5233 & 5424 & 5228 & 4436 & 4473 \\
\hline CDSs on plasmid & 44 & NA & NA & NA & NA & NA \\
\hline Hypothetical proteins & 1091 & 1209 & 1292 & 1214 & 1101 & 991 \\
\hline $\begin{array}{l}\text { Average CDS length } \\
\text { (nt) }\end{array}$ & 927 & 923 & 904 & 917 & 834 & 899 \\
\hline Coding (\%) & $87.8 \%$ & $86.2 \%$ & $85.6 \%$ & $85.8 \%$ & $78.4 \%$ & $85.4 \%$ \\
\hline rRNA & 9 & 9 & 9 & 9 & 9 & 9 \\
\hline tRNA & 52 & 52 & 52 & 51 & 52 & 53 \\
\hline contigs & 1 & 1 & 1 & 1 & 1 & 1 \\
\hline
\end{tabular}

NA not applicable, refers to strains in which no plasmids are naturally present

\section{Phylogenetic analysis}

A phylogenetic tree based on 233 protein-coding genes (Fig. 1A) revealed that C. fungivorans and $C$. pratensis are more closely related to each other than to $C$. arenae. Similar clustering was observed based on phylogenetic trees generated with whole genome fragments (200 bp fragment sizes) (Fig. 1B) and 16S rRNA (Additional file 1: Fig. S1) where each strain falls into its respective species clade. 
A

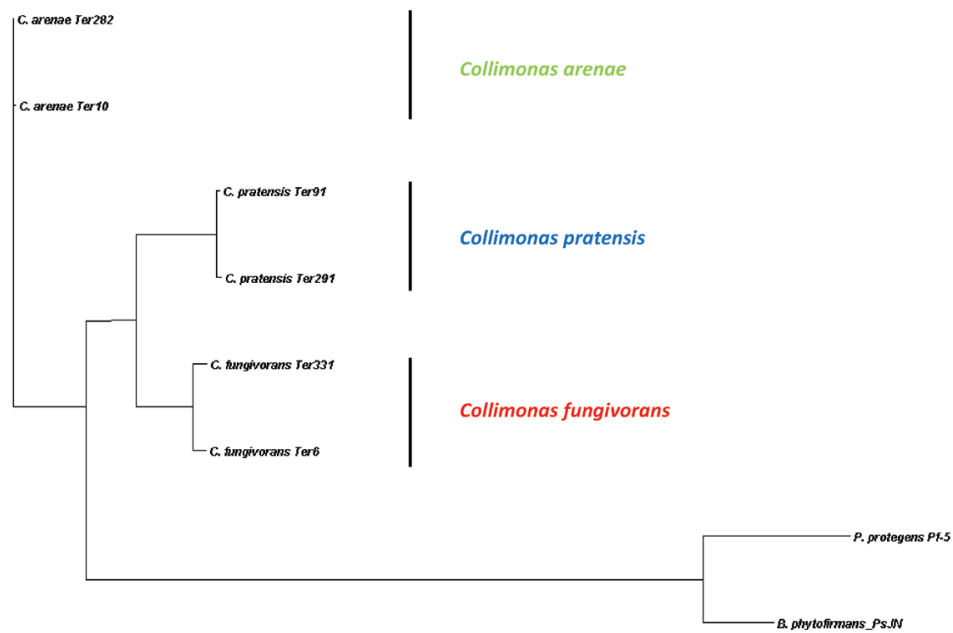

B

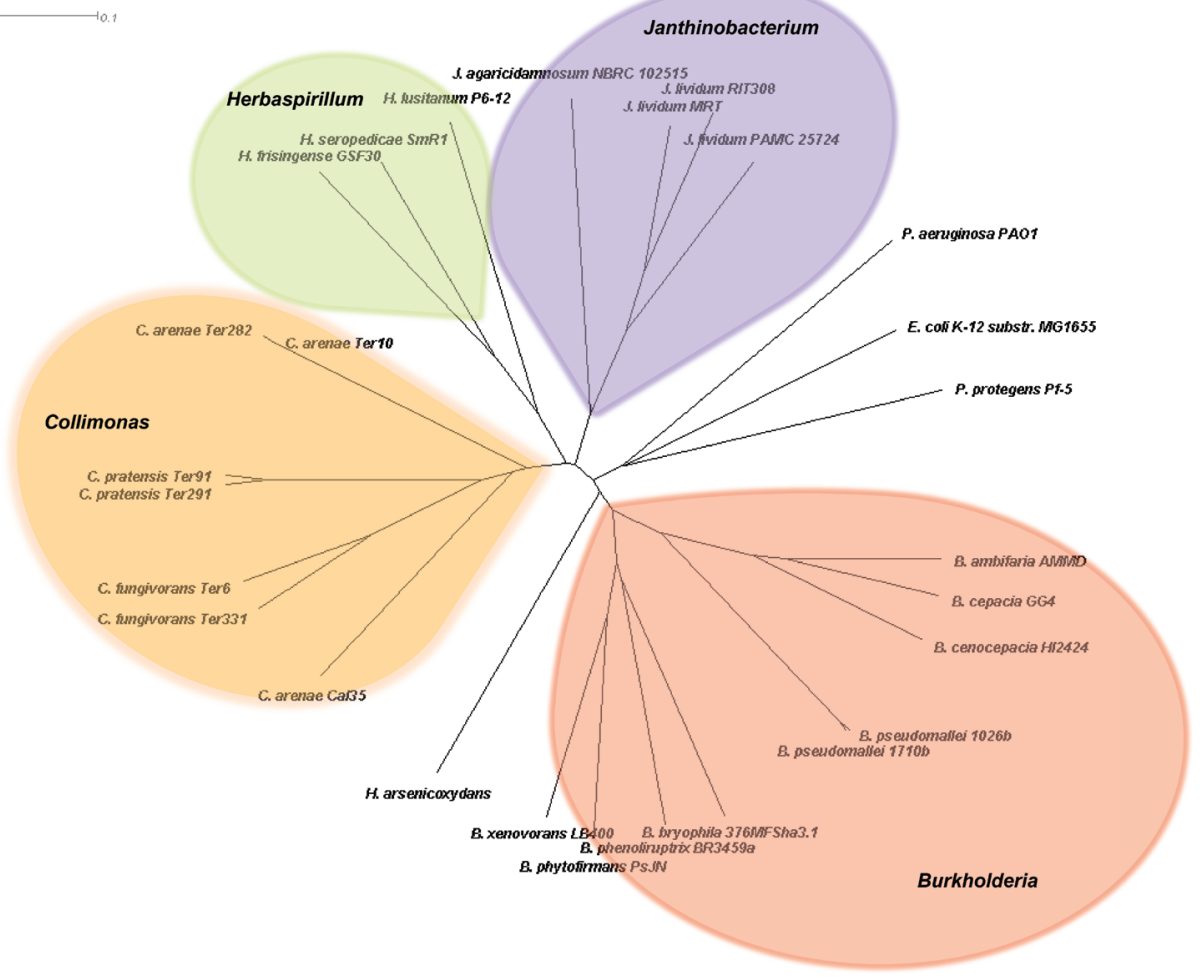

119 / Chapter 5 
Fig. 1 I Whole genome phylogeny of the six Collimonas genomes. (A) Neighbor-joining tree based on concatenated sequences for 233 protein encoding genes. Pseudomonas protegens Pf-5 and Burkholderia phytofirmans PsJN were used as outgroup. Bootstrap values are shown on branches. (B) Phylogenetic tree based on a fragmented alignment using BLASTN made with settings 200/100. A dendrogram was produced in SplitsTree 4.13.1 (using neighbor joining method) made from a Nexus file exported from Gegenees. Burkholderia, Janthinobacterium and Herbaspirillum were set as outgroups. Bootstrap values are of all the branches are 100, for clarity reason, not shown in the Figure.

\section{Core and Pan-genome analysis}

A core genome containing 2339 predicted orthologous groups was identified for the six Collimonas strains based on the all-vs-all BLASTp search (Fig. 2A). This core genome represents 43.1 to $52.7 \%$ of the predicted ORF's of each strain (Fig. 2A), illustrating a large degree of genomic diversity between these strains. Each of the six genomes includes 125 to 835 orthologous groups that are unique (Fig. 2A). Species core orthologous clusters and strain-specific unique clusters within the three Collimonas species were examined, respectively (Fig. 2B-D). In the three species, 5868, 5810 and 4546 orthologous clusters were identified and of these, 3859, 4194 and 3829 orthologs were present as the species core genome for $C$. fungivorans, $C$. pratensis, C. arenae, respectively (Fig. 2B-D). A core-pan genome evolution plot summarizing the variability in each possible combination of Collimonas species shows that the number of unique (singleton) gene clusters is stable. The variable gene clusters are increasing and the core gene clusters are decreasing (Fig. 2E). In order to determine the differences in functions encoded by the core and variable genome of each strain, the proportion of proteins in each COG (Clusters of Orthologous Groups) was plotted versus the COG function. The relative abundance of almost all the COG categories was higher in the core genome of the six strains than in the variable genome. This was not the case for COG categories N (Cell motility), $\cup$ (Intracellular trafficking, secretion, and vesicular transport) and proteins that cannot be assigned in COG categories (data not shown) where the proteins were more abundant in variable genomes for C. fungivorans and C. arenae (Fig. 3A). This is most probably due to the fact that a flagellar and chemotaxis-related gene cluster is only present in these two species but not in C. pratensis (Additional file 2: Table S1). This finding is consistent with the observed reduced swimming motility of C. pratensis Ter91 and Ter291 as compared to the other four strains (Fig. 3B). 


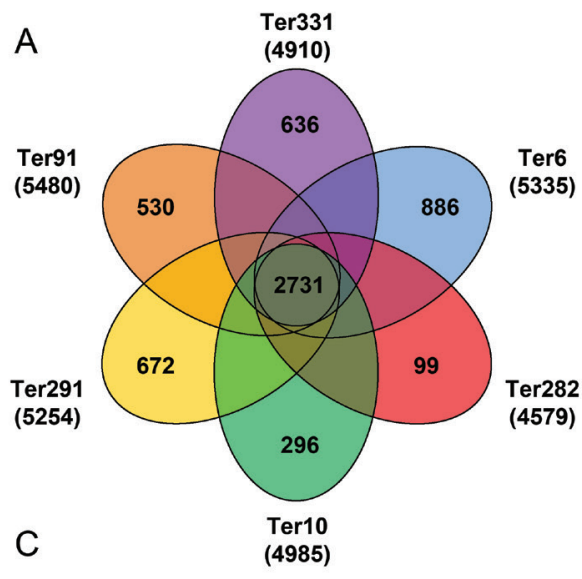

B

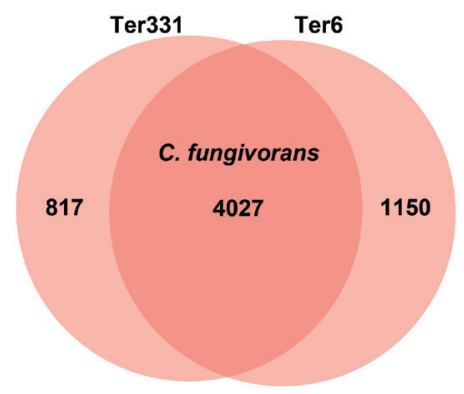

D
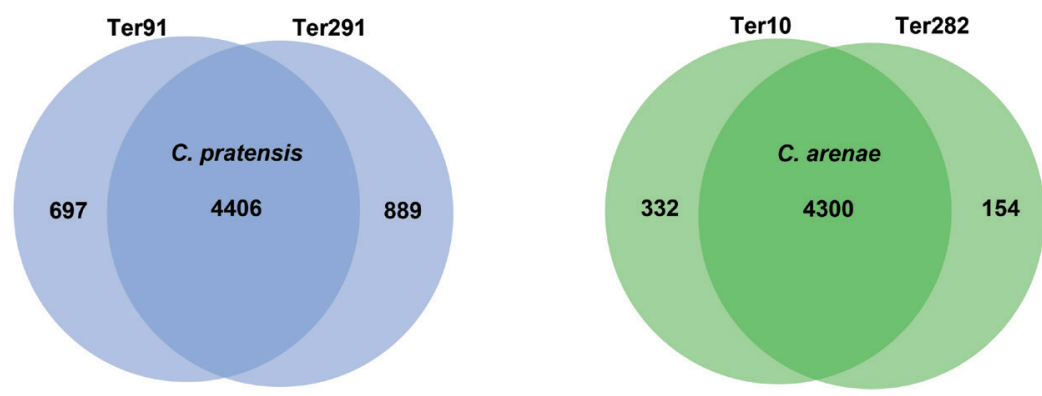

E

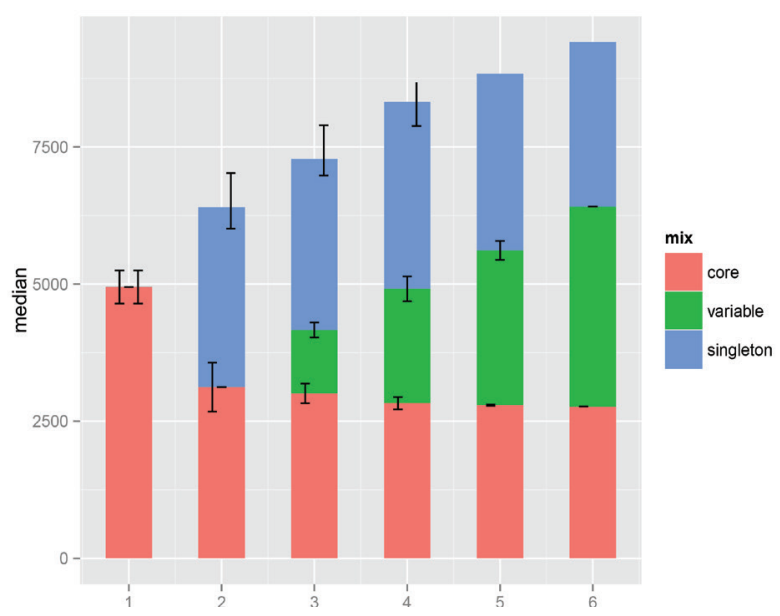

Fig. 2 I The pan-core genome of Collimonas strains. The venn diagrams illustrate the number of shared and unique genes based on clusters of orthologs. (A) Venn diagram showing numbers of speciesspecific genes commonly found in each genome of each species, (non-overlapping of each oval) and Collimonas core orthologous gene number (in the centre). The total number of protein coding genes 
within each genome is listed below the strain name. (B) Venn diagram showing numbers of unique orthologues genes in C. fungivorans strains. (C) Venn diagram showing numbers of unique orthologues genes in C. pratensis strains. (D) Venn diagram showing numbers of unique orthologues genes in $C$. arenae strains. (E) Core- and pan-genome as function of the number of genomes taken from the six Collimonas genomes in this study. The number of shared and strain specific gene clusters between strains depends on which combinations of strains (x-axis). Specific singleton gene clusters (blue bars) occur only in one strain, variable gene clusters (green bars) occur in more than one but not all strains and core gene clusters (red bars) occur in all strains of a given combination. Error bars represent the standard deviation in the core- (left error bar), variable- (middle error bar) and singleton- (right error bars) gene clusters.

A

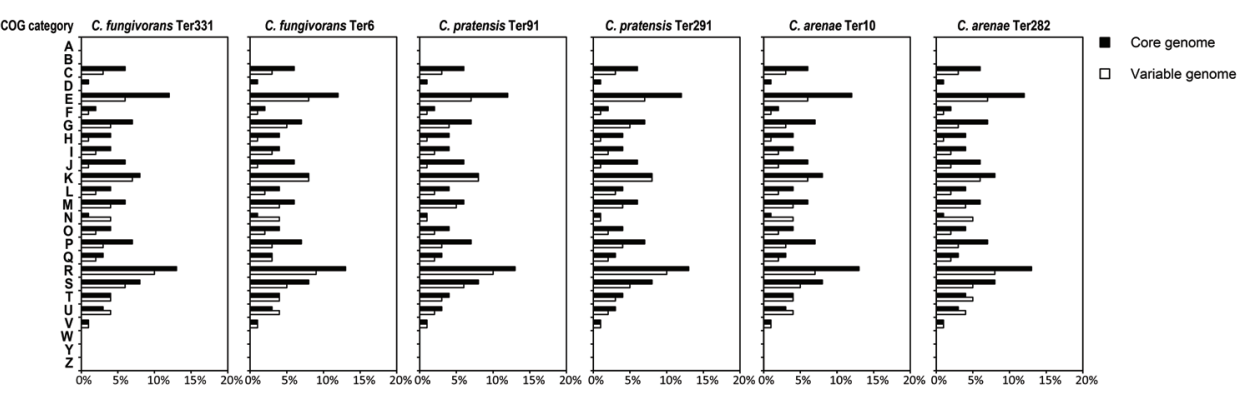

A: RNA processing and modification, B: Chromatin structure and dynamics, C: Energy production and conversion, D: Cell cycle control, cell division, chromosome partitioning E: Amino acid transport and metabolism, F: Nucleotide transport and metabolism, G: Carbohydrate transport and metabolism, $\mathrm{H}$ : Coenzyme transport and metabolism, I: Lipid transport and metabolism, J: Translation, ribosomal structure and biogenesis, K: Transcription, L: Replication, recombination and repair,

M: Cell wall/membrane/envelope biogenesis, N: Cell motility, O: Posttranslational modification, protein turnover, chaperones, $\mathrm{P}$ : Inorganic ion transport and metabolism,

$\mathrm{Q}$ : Secondary metabolites biosynthesis, transport and catabolism, R: General function prediction only, S: Function unknown, T: Signal transduction mechanisms,

U: Intracellular trafficking, secretion, and vesicular transport, V: Defense mechanisms, W: Extracellular structures, Y: Nuclear structure, Z: Cytoskeleton

B

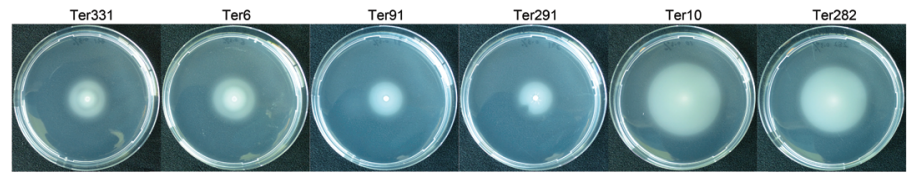

C

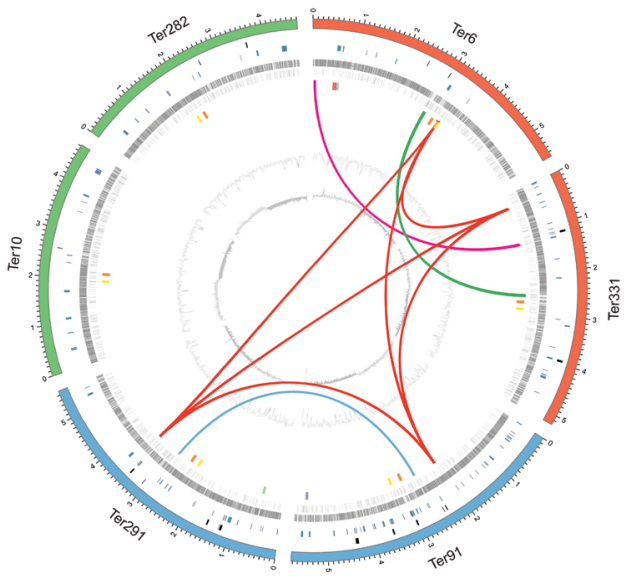

Fig. $\mathbf{3}$ / Distribution of orthologous genes based on COG category in each Collimonas strain. (A) The percentage of orthologous genes assigned by COG category in the core genome (black bars) and 
the variable genome (white bars). (B) Swimming motility of Collimonas strains Ter331, Ter6, Ter91, Ter291, Ter10 and Ter282 on soft (0.3\% [wt/vol]) agar plates. (C) Comparative genome content of the six Collimonas strains. From the outside to the inside circles: Chromosomes of all six strains (red: $C$. fungivorans, blue: C. pratensis, green: C. arenae). Phages/phage-like regions: black bars. Genomic islands: dark blue bars. Genes in the forward (dark grey) direction, genes in the reverse (light grey) direction, G + C content (dark grey and light grey), GC skew (dark grey: negative values, light grey: positive values). Universal and unique gene clusters are indicated with colored bars (orange: Ornibactin, yellow: Phytoene, red: T3pks-nrps (Ter6), light blue: 2-aa NRPS-1 (Ter6), purple: 2-aa NRPS-2 (Ter291), light green: Nrpst1pks (HSAF, Ter91)). Shared secondary metabolite gene clusters are indicated with colored lines (deep pink: Collimomycin, dark red: NLP, dark green: Tripropeptin A)

Whole genome alignments of the six strains were performed to obtain information on the nucleotide level synteny (Additional file 1: Fig. S2). These alignments revealed a very high level of synteny when genomes of strains from the same species were compared (Additional file 1: Fig. S2A-C), but many rearrangements and inversions were observed between the genomes of all strains (Additional file 1: Fig. S2D).

\section{Genomic islands (GIs), bacteriophages and CRISPRs}

Genomic islands (GIs) are mobile genetic elements acquired by horizontal transfer, which carry multiple genes that are typically involved in pathogenesis or symbiosis. The Collimonas genomes carry 7 to $47 \mathrm{Gls}$ ranging from $4.0 \mathrm{~kb}$ to $64 \mathrm{~kb}$ in size (Fig. 3C). Altogether, the six Collimonas genomes have 139 genomic islands. The large numbers of Gls indicate a complex history of genes recombination and horizontal transfer between bacterial relatives. The genomes of all strains contain one to five possible phages, each ranging in size from 7.0 to $59.9 \mathrm{~kb}$. In total, the six genomes have 18 phages with some of the phages falling into the GIs (Fig. 3C). CRISPRS (ㄷustered Regularly Interspaced Short Palindromic Repeats) are DNA loci that are involved in prokaryotic immunity to phage infection. Putative CRISPRs were identified using the CRISPRsFinder program (Grissa et al., 2007)2007. Two confirmed CRISPRs were present only in C. fungivorans Ter6 genome. For the other five genomes, no or only questionable CRISPRs were found (data not shown).

\section{Secretion systems}

In gram-negative bacteria, type II secretion systems (T2SSs) are the most ubiquitous secretion systems used by bacteria to export many extracellular enzymes. T2SSs are conserved and known as a two-step process: proteins are translocated across the inner membrane by the Sec or Tat pathway, and then transported from the periplasm 
to the exterior by an outer membrane secretin (Cianciotto, 2009). SecABDEFY, yajC, yidC, ftsY, and ffh and tatABC encoding genes for Sec and Tat pathways respectively were found to be present in all Collimonas genomes (Fig. S3A; Table S2). The outer membrane secretion unit of the T2SS in the Collimonas genomes resembles the Gsp system which contains one gene cluster gspD-N, responsible for secretion of protease, lipase, and phospholipase C in Burkholderia (DeShazer et al., 1999)1999 (Fig. S3A; Table S2). A newly described subtype of T2SS, tad locus (tight adherence) (Tomich et al., 2007) was identified in all six genomes. The tad locus encodes the machinery required for the assembly of adhesive Flp (fimbrial low-molecule-weight protein) pili and is necessary for bacterial adhesion to surfaces, biofilm formation, and pathogenesis as shown for Aggregatibacter actinomycetemcomitans (Planet et al., 2003), Haemophilus (Nika et al., 2002), Pseudomonas (Durand et al., 2003), Yersinia etc. (Tomich et al., 2007). For the mycophagous behavior of Collimonas, the adhesion to fungal hyphae might be of prime importance (Hoppener-Ogawa et al., 2009) and the presence of the T2SS tad locus in all strains indicates that it may be an essential trait for the mycophagy lifestyle of the Collimonas species.

Type III secretion systems (T3SSs) are used by various Gram-negative bacteria to inject effector proteins into host cells, promoting either mutual benefit or pathogenesis (Cornelis \& Wolf-Watz, 1997, Galan \& Collmer, 1999) and have been described as important for bacterial interaction with fungi (Warmink \& van Elsas, 2008). Three Collimonas strains Ter331, Ter6 and Ter91 carry hrp-hrc1 family gene clusters of T3SS and a second T3SS (Fig. S3A; Table S2). The T3SSs have crucial role in the virulence of plant and human pathogens (Tseng et al., 2009). However, their functions in non-pathogenic bacteria are still poorly understood, with indications that mutation of T3SSs in a plant-growth promoting bacteria P. fluorescens SBW25 resulted in a significant reduction in colonization of the root tips of sugar beet seedlings (Jackson et al., 2005). They have also been suggested to be involved in bacterial-fungal interactions, to facilitate bacteria migration along fungal hyphae (Warmink \& van Elsas, 2008). For Collimonas, the T3SSs may be important to inject membrane disturbing compounds into the fungal host to get access to nutrients inside the fungal hyphae (Leveau et al., 2010)

Type VI secretion systems (T6SSs) are conserved and prevalent in Gramnegative bacteria. They are known to be involved in competition, predation and inter-specific bacterial interactions (Schwarz et al., 2010, Records, 2011, Russell 
et al., 2011). In this study, only in C. fungivorans and C. pratensis strains a cluster of genes encoding T6SS was identified, but not in the $C$. arenae species. This may indicate that horizontal gene transfer events or evolutionary genes loss have occurred. Furthermore in C. fungivorans Ter331, part of the T6SS is located on a genomic island.

Further functional studies are needed to determine the exact role of these secretion systems for Collimonas lifestyle and in particular for the attack of fungi.

\section{Signal transduction systems}

Signal transduction systems play important roles for many bacteria enabling them to detect and respond to changes and stresses in the environment (Stock et al., 2000). Each Collimonas genome encodes 267 to 365 one-component systems (1CSs) which are the majority of signal transduction systems in prokaryotes (Ulrich et al., 2005) and 90 to 109 two component system (TCSs) (Table S3). Additionally, 9 to 29 genes involved in chemotaxis systems were found. Extracytoplasmic function (ECF) sigma factors which comprise the largest group among the $\sigma 70$ family (Ulrich et al., 2005) were also found in all Collimonas genomes with numbers ranging from 9 to 11. Higher numbers of signal transduction system were predicted in C. fungivorans and $C$. pratensis as compared to $C$. arenae. The overall high number of genes related to signal transduction systems (8-9\% of predicted sequences in the six Collimonas genomes) suggest that Collimonas possess the ability to sense environmental signals and cues important for their growth, survival and interactions in the heterogeneous and complex soil environment.

Our genomic analysis revealed that all six Collimonas genomes contain two QS genes: one autoinducer gene and one luxR-type transcriptional regulator. They show 40\% homology to the CepIR system from Burkholderia (Table S4) which is known to regulate protease, lipases, chitinases and some other exoenzymes production (Lewenza et al., 1999, Aguilar et al., 2003). Quorum sensing assay performed with the indicator strain C. violaceum CV026 revealed clear short chain AHL production in C. fungivorans Te331, Ter6 and C. pratensis Ter91, Ter291 strains, but no or traced amount in C. arenae Ter10 and Ter282 strains (Fig. S3B). In all strains, AHL production was detected when A. tumefaciens NT1 was used as QS bioreporter (Fig. S3C). 


\section{Secondary metabolome of Collimonas strains}

Bacteria often produce set of secondary metabolites with antimicrobial properties important for competition and survival in competitive environments. In Collimonas, the secondary metabolites are thought to play an important role for enabling mycophagous growth, namely by disturbing the fungal membrane integrity (Leveau et al., 2010). Although Collimonas was suggested to represent a valuable resource for the discovery of novel molecules and enzymes (Leveau et al., 2010), to date only two antimicrobial compounds were described for this genus, namely violacein and collimomycin (Hakvag et al., 2009, Fritsche et al., 2014). Violacein was identified in the Collimonas CT strain isolated from an aquatic environment and revealed antibacterial activity against Micrococcus luteus (Hakvag et al., 2009). However, in the six Collimonas genomes here, no genes encoding violacein were identified.

Collimomycin is a polyacetylenic compound with alternating triple and single carbon-carbon bonds (Fritsche et al., 2014) which is produced by C. fungivorans Ter331 and was shown to have strong antifungal activities (Fritsche et al., 2014). The corresponding biosynthesis cluster K, is only partially present in C. fungivorans Ter6, and completely absent in the other four genomes (Fig. 3C, Table S5). Moreover, the part of the collimomycin gene cluster that is present only in C. fungivorans Ter331 is located in a genomic island suggesting horizontal gene transfer event.

\section{Exoenzymes}

Exoenzymes are extracellular enzymes produced inside the cell, then released outside the cell to begin the process of extracellular digestion. Exoenzymes may be of importance for Collimonas nutrient acquisition, microbial interactions, mycophagy and weathering.

\section{Chitinases}

Chitin is a major component of fungal cell walls and is a homopolymer of $\mathrm{N}$-acetyl-D-glucosamine (GlCNAc). Chitinases are able to hydrolyze the 1,4-betalinkages of chitin (Rabea et al., 2003). Two loci A and B of chitinase biosynthesis and transport were found in the genome of C. fungivorans Ter331 (Fritsche et al., 2008). Here, we revealed that the other five Collimonas genomes also contain a complete set of genes in these two loci (Table S6). This is in line with the previous observation based on comparative genomic hybridization study (Mela et al., 2012) 
and indicates that acquisition of these genes occurred before Collimonas speciation. Phenotypic evaluation for chitinase production of these strains was confirmed by halo formation on water-agar plates containing colloidal chitin (de Boer et al., 2004). Bacterial chitinase activity has often been reported to be responsible for antifungal properties (Chernin et al., 1995, Kobayashi et al., 2002, Chang et al., 2003, Dahiya et al., 2005). However, mutants in the chitinase loci of C. fungivorans Ter331 showed no difference during in vitro antagonism test (Fritsche et al., 2008). This suggested that chitinases might not contribute solely to the antifungal activities of Collimonas. The antifugal activities might be coupled with the production of other secondary metabolites. Indeed, when supplementing chitinase inhibitor allosamidin, the growth of Collimonas on fungi was decreased, suggesting potential contribution to its mycophagous ability (De Boer et al., 2001).

\section{Phospholipases}

Phospholipases are a group of enzymes that catalyze the cleavage of phospholipids. Two major phospholipase activities can be defined by the site of cleavage, namely in the hydrophobic diacylglycerol moiety (PLA) or in the polar head group of the amphipathic phospholipid (PLC and PLD) (Schmmiel and Miller, 1999). In general, 11 to 15 phospholipases from four different groups: phospholipase A1, phospholipase C, phospholipase D and patatin phospholipase were detected in the six Collimonas genomes (Fig. 4A; Table S7). Phospholipases are considered virulence factors for pathogenic bacterial species which cause tissue destruction, lung infections, hemolysis etc. (Schmiel \& Miller, 1999). Given the cleavage properties of phospholipids we speculate that they might be involved in nutrient acquisition via fungal membrane disturbing activities as well as in defense against competitors. 


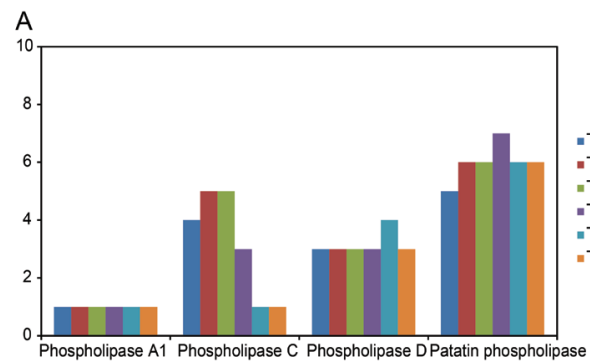

C

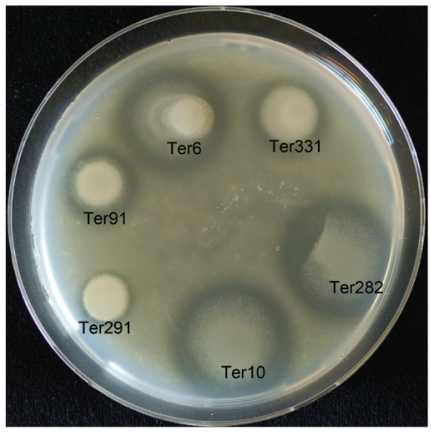

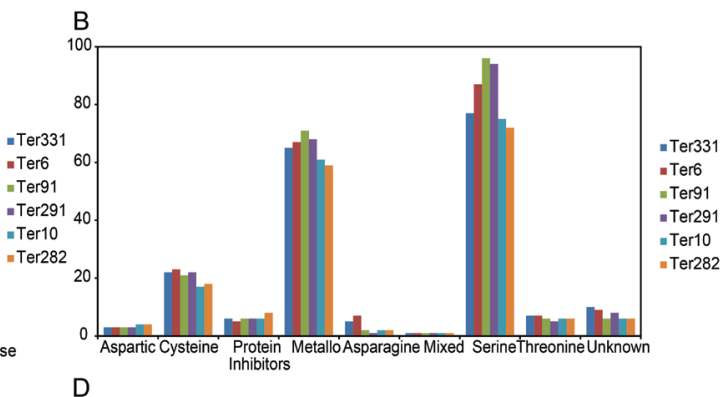

D

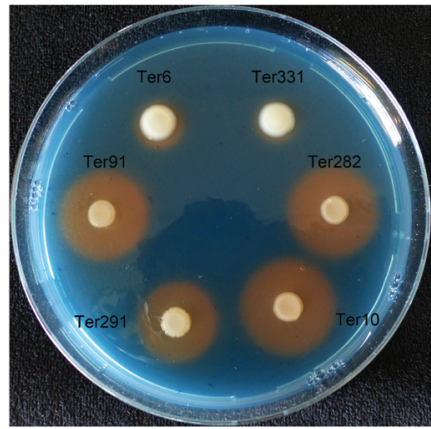

E

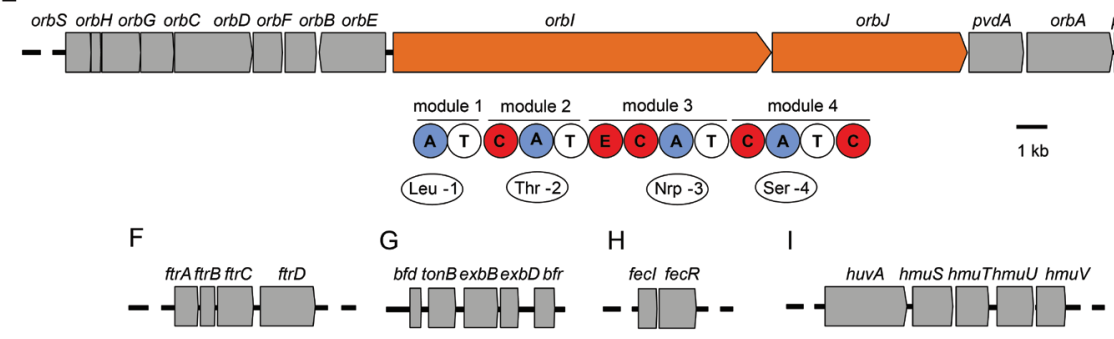

Fig. 4 / (A) Identification of phospholilpases in the six Collimonas genomes with PFAM signatures of the different phospholipase groups. (B) Identification of peptidases in the six Collimonas genomes as inferred from MEROPS 9.12 database. (C) Extracellular protease activity of Collimonas strains Ter331, Ter6, Ter91, Ter291, Ter10 and Ter282. A halo indicates extracellular protease production. (D) Siderophore production of Collimonas strains Ter331, Ter6, Ter91, Ter291, Ter10 and Ter282 plated on a CAS plate. An orange halo indicates of siderophore production. Gene clusters involved in iron acquisition in Collimonas strains. Five loci are presented: (E) Ornibactin locus. Underneath the genes are the module and domain organization of orb/ and orbJ. The domains are as follows: C, condensation; A, adenylation; $T$, thiolation; and E, Epimerization. Underneath the domains are the amino acids that are incorporated into the peptide moiety. The number associated with the amino acid refers to the position of the amino acid in the peptide chain. (F) $\mathrm{ftr}{ }_{b c c} A B C D$ locus. (G) bfr (bacterioferritin) encoding gene and adjacent genes bfd and tonB-exbB-exbD cluster. (H) feclR operon. (I) hmu operon (or bhu Burkholderia haem uptake operon) 


\section{Peptidases}

In the six Collimonas genomes, 176 to 212 peptidases were predicted and nine families of proteolytic enzymes were identified (Fig. 4B). Among them, serine and metallo peptidases are the two dominant families. The Collimonas strains in our study were tested positive for exoprotease production (Fig. 4C). Serine proteases are one of the most abundant groups of proteolytic enzymes found in all living organisms (Page \& Di Cera, 2008) and in prokaryotes, serine proteases are involved in several biological processes associated with cell signaling, defense response and development (Gottesman, 2003, Hengge \& Bukau, 2003, Jenal \& Hengge-Aronis, 2003). Furthermore, serine protease can be involved in regulating the biosynthesis of lipopeptides, which can play a role in the suppression of other microbes (de Bruijn \& Raaijmakers, 2009).

\section{Iron acquisition}

Siderophores are low molecular weight, high-affinity iron chelating compounds produced by microorganisms under iron limited conditions and function in solubilization, transport and storage of iron (Chu et al., 2010, Hider \& Kong, 2010). Siderophore production can act as an antagonistic mechanism by scavenging limited iron from the soil environment, thereby reducing the amount of available iron for other organisms. Our analysis revealed that all six strains encode biosynthesis clusters which resemble ornibactin (Fig. 4E), a siderophore synthesis cluster of Burkholderia cenocepacia (Agnoli et al., 2006). Siderophore production was confirmed for all the six Collimonas strains, albeit with different production efficiency (Fig. 4D).

Next to siderophore production, other mechanisms of iron acquisition were reported. For example recently, a novel alternative siderophore-independent iron uptake system was identified in Burkholderia, named $\operatorname{tr}_{\text {bcc }} A B C D$ locus. This ftrABCD operon was identified in all six Collimonas genomes (Fig. 4F), indicating that there are more strategies for iron uptake besides ornibactin production. Moreover, we found genes coding for the production of bacterioferritin, a type of iron-storage protein (Andrews, 1998). The gene is often adjacent to genes encoding a small [2Fe-2S]-ferredoxin Bfd. Downstream of the bfd gene is the tonB-exbB-exbD cluster which encodes a system to transduce cellular energy to outer-membrane receptors for siderophores and haemin (Schalk et al., 2004). The bacterioferritin 
encoding gene bfr and adjacent genes bfd and tonB-exbB-exbD cluster were found in the six Collimonas genomes (Fig. 4G). Furthermore, a feclR operon was also found in all six genomes (Fig. 4H). It is known that the transcription of genes for ferriccitrate transport in E. coli requires Fecl, and FecR, a cytoplasmic membrane protein encoded by the second gene in the Fur-repressed feclR operon which transmits an external iron signal to the cytoplasmic Fecl protein (Braun et al., 2003)2003. A haem uptake system similar to that of Burkholderia cenocepacia 12315 was found in the genomes of $\boldsymbol{C}$. fungivorans Ter6 and C. pratensis Ter91, it is encoded by the hmu operon (Holden et al., 2009). This operon is comprised of five genes (Fig. 4l) and was suggested to be Fur-mediated. Furthermore, fungi are known to produce haem (Franken et al., 2012), therefore, it is plausible that Collimonas are able to use the fungal haem as source of iron.

\section{NRPS and PKS-NRPS genes encoding metabolites}

\section{Lipopeptides}

Lipopeptides (LPS) are compounds composed of a lipid tail with a linear or cyclic oligopeptide (Raaijmakers et al., 2010). They exhibit surfactant, antimicrobial, antipredation, and cytotoxic properties (Raaijmakers et al., 2006, Raaijmakers \& Mazzola, 2012). LPs are synthesized in bacteria by large nonribosomal peptide synthetases (NRPSs) via a thiotemplate process. The structural diversity of the LPS is due to differences in the length, composition of the fatty acid tail, and the number, type and configuration of the amino acids in the peptide moiety. Via in silico analysis, we identified gene clusters for LP biosynthesis of tripropeptin A in the genomes of C. fungivorans Ter331 and Ter6 (Fig. 5A). Although the structure of tripropeptin A has been known for more than a decade (Hashizume et al., 2001, Hashizume et al., 2004), genetic analysis of its biosynthesis was only recently reported (Medema et al., 2014). Our study revealed that three NRPS genes $\operatorname{trpA}, \operatorname{trp} B$, $\operatorname{trp} C$ are organized in a single-operon (Fig. 5A). The trpA gene, encodes an NRPS with the first five modules, and $\operatorname{trp} B$, $\operatorname{trp} C$ encode one and two modular NRPS, respectively. The wild type $C$. fungivorans Ter331 and Ter6 possess antibacterial activity against Staphylococcus aureus but not the other four strains from C. pratensis and C. arenae (Fig. 5B). The site-directed $\triangle$ trpA mutant of $C$. fungivorans Ter331 lacks this antagonism (Fig. 5B) which indicates that tripropeptin $\mathrm{A}$ is indeed involved in this antibacterial activity and this is a unique trait for the C. fungivorans strains. 
Another 16.9 kb NRPS gene was found in C. fungivorans Ter331, Ter6 and C. pratensis Ter91, Ter291 strains (Fig. 3C; Fig. 5C). This gene is composed of five amino acids without hits to the known lipopeptides indicating a new lipopeptide. Collimonas strains are well known for their ability to suppress a range of fungi and oomycetes (De Boer et al., 1998, Opelt \& Berg, 2004, Adesina et al., 2007, Fritsche et al., 2014). However, our study revealed that the six Collimonas strains possess different extent of in vitro suppression against fungal and oomycetal pathogens (Fig. 5D; Table S8). When the new lipopeptide was knocked out in C. fungivorans Ter331 by site-directed mutagenesis, reduced suppression was observed (Fig. 5D). This indicated that the new lipopeptide contributes to antimicrobial activity against both fungal and oomycetal pathogens. For the $C$. arenae species, no new lipopeptide was found in the genome, but the strains still revealed suppressing activities against fungal and oomycetal pathogens (Table S8). This indicated that apart from new lipopeptide, there is/are other factor(s) involved in antimicrobial activity or that the suppression activity might be due to synergistic effect rather than a single compound. Thus, the different types of lipopeptide produced by the different Collimonas strains may partly explain the variability in the fungal inhibition behavior of these strains. The variability is also reflected in the unknown orphan gene clusters described below. 
A

$--\operatorname{trpA} x \operatorname{trpB} \sqrt{\operatorname{trp} C}-\overline{1 \mathrm{~kb}}$

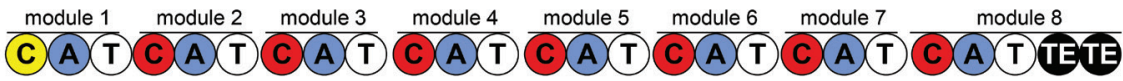

Thr-1 Pro-2 Pro-3 Arg-4 Asp-5 Ser-6 Pro-7 Asp-8

B
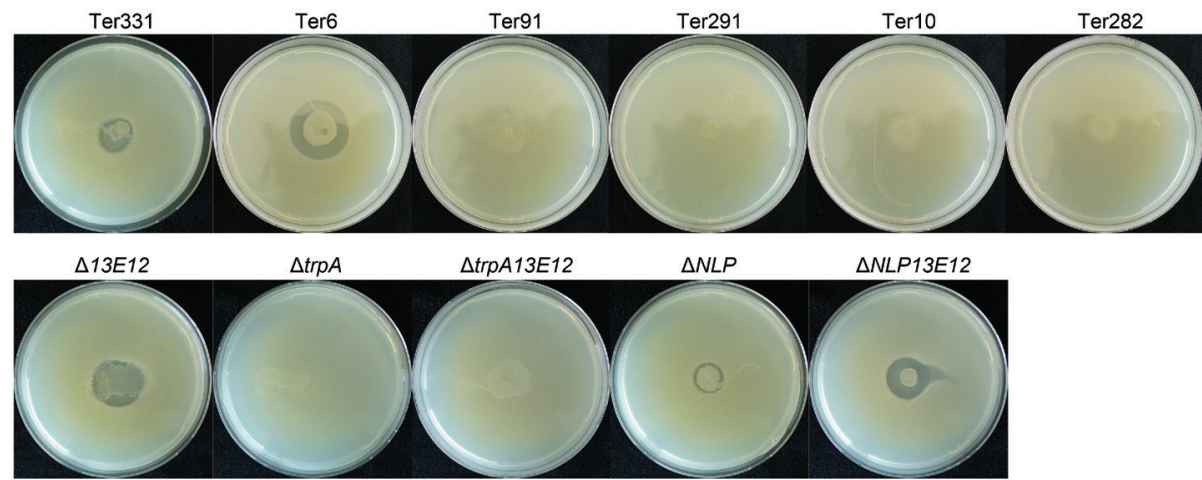

C

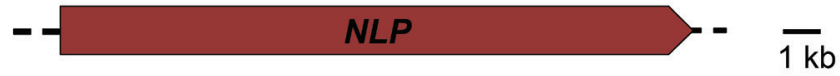

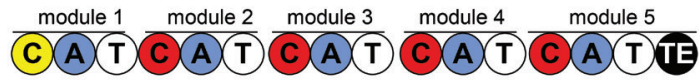

Thr-1 Thr-2 Nirp-3 Ser-4 Ne-5

D

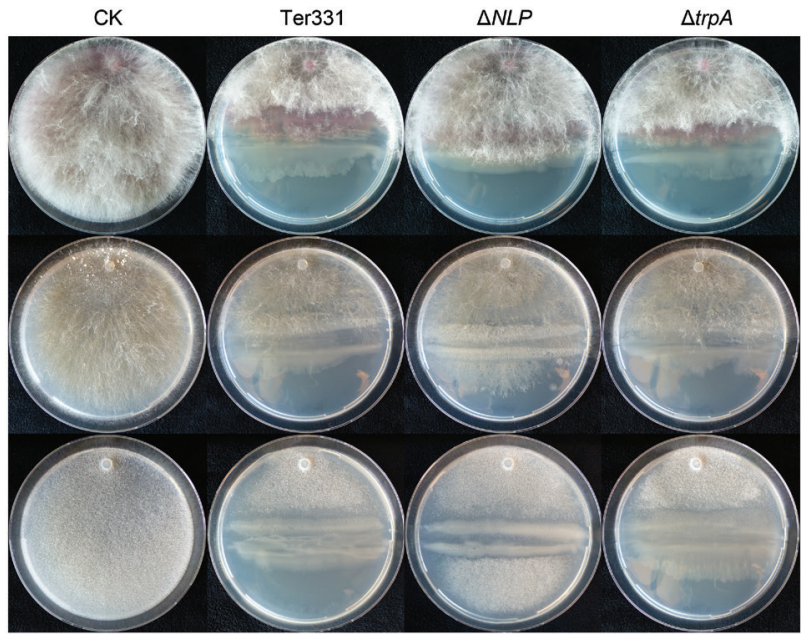

Fusarium

culmorum

Rhizoctonia

solani

Saprolegnia

parasitica 
Fig. 5 / Biosynthetic gene cluster and antibacterial activity associated with tripropeptin A production by the Collimonas stains. (A) Organization of the gene cluster and predicted amino acid composition of the Tripropeptin A in C. fungivorans Ter331 and Ter6 genomes. Underneath the genes are the module and domain organization of $\operatorname{trp} A, \operatorname{trp} B$ and $\operatorname{trp} C$. The domains are as follows: $C$, condensation; $\mathrm{A}$, adenylation; $\mathrm{T}$, thiolation; and $\mathrm{TE}$, thioesterification. Underneath the domains are the amino acids that are incorporated into the LP peptide moiety. The number associated with the amino acid refers to the position of the amino acid in the LP peptide chain. The black triangle indicates the position of the Gm cassette insertion in the trpA gene. (B) Antibacterial activity associated with tripropeptin A production. Strains Ter331, Ter6, $\triangle 13 E 12$ mutant (deficient in collimomycin biosynthesis), $\triangle N L P$ mutant (deficient in the new lipopeptide biosynthesis) and $\triangle$ NLP13E12 mutant (deficient in bothnew lipopeptide and collimomycin biosynthesis) exhibited inhibition against Staphylococcus aureus via overlay assay. $\triangle t r p A$ mutant of $C$. fungivorans Ter331 (deficient in tripropeptin A biosynthesis)- loss in the inhibition activity. Biosynthetic gene cluster and antifungal/oomycetal activities associated with new lipopeptide production by the Collimonas stains. (C) Organization of the gene cluster and predicted amino acid composition of the new lipopeptide in C. fungivorans Ter331, Ter6 and C. pratensis Ter91, Ter291 genomes. Underneath the genes are the module and domain organization of NLP. The domains are as follows: C, condensation; A, adenylation; T, thiolation; and TE, thioesterification. Underneath the domains are the amino acids that are incorporated into the LP peptide moiety. The number associated with the amino acid refers to the position of the amino acid in the LP peptide chain. The black triangle indicates the position of the Gm cassette insertion in the $n / p$ gene. (D) Antifungal/oomycetal activities associated with new lipopeptide production. Strain Ter331 which has new lipopeptide biosynthetic clusters exhibited inhibition against Fusarium culmorum, Rhizoctonia solani and Saprolegnia parasitica. Mutant $\triangle N L P$ of Ter331 deficient in new lipopeptide biosynthesis abolished the inhibition activities. CK is control grew without bacteria

\section{Unknown orphan NRPS and PKS-NRPS hybrid gene clusters}

Within the genomes, four orphan gene clusters were identified. Two different 2-amino acids (2-aa) NRPS genes were found in Ter6 and Ter291 respectively (Table S9; Fig. S4B, C). Next to this, a 54.5 kb unknown T3PKS-NRPS gene cluster was discovered in the genome of Ter6 (Fig. S4A). Another $10.6 \mathrm{~kb}$ NRPS-T1PKS gene cluster in Ter91 (Fig. S4D) resembles clusters encoding the Heat-stable antifungal factor (HSAF), also referred to as dihydromaltophilin, produced by Lysobacter species (Yu et al., 2007, Lou et al., 2011). HSAF exhibits inhibitory activities against a wide range of fungal species by disrupting the polarized growth or the biosynthesis of a distinct group of sphingolipids of fungi (Yu et al., 2007, Lou et al., 2011).

\section{Terpenes}

Terpenes are a diverse family of primary and secondary metabolites which were mostly studied in plants and fungi (Trapp \& Croteau, 2001). Many terpenes of plant origin are known to be active against a wide variety of microorganisms, including 
gram-positive, gram-negative bacteria and fungi (Tholl, 2006), however to date there are only few reports on antimicrobial activity of terpenes from microbial origin (Gurtler et al., 1994, Zhao et al., 2008). In a previous study, four monoterpenes ( $y$-terpinene, 1S-a-pinene, $\beta$-pinene and $\beta$-myrcene) were detected in the headspace of $C$. pratensis strains Ter91 (Garbeva et al., 2014). Here, these monoterpenes were tested individually and as a mixture for their antimicrobial activity. The $\beta$-pinene exhibited inhibition against Staphylococcus aureus and Rhizoctonia solani and the mixture of all monoterpenes revealed inhibition against these two pathogens and also to E. coli (Fig. 6A).

A

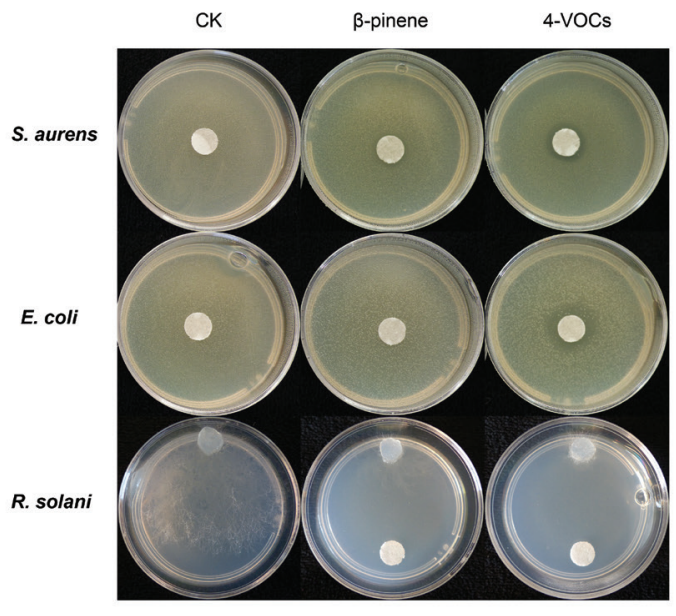

C

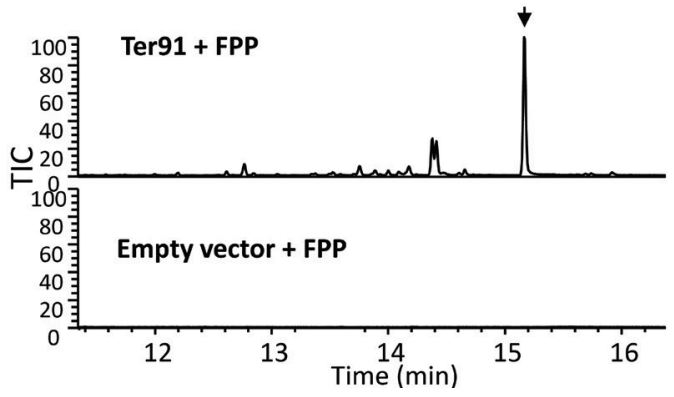

B

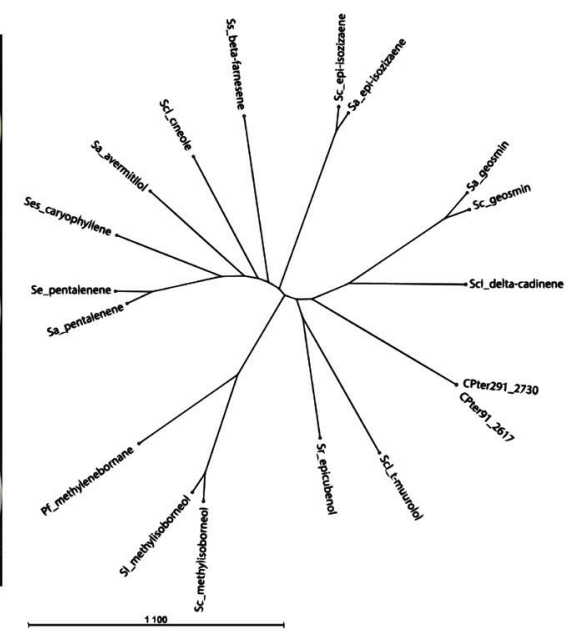

Peak RT 15.17

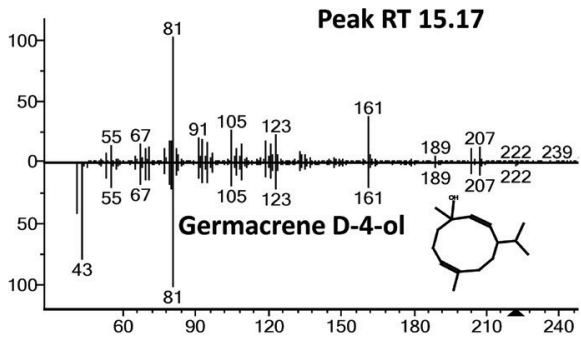

Fig. $\mathbf{6}$ / (A) Antimicrobial activities of pure $\beta$-pinene and 4-VOCs (mix of $\beta$-pinene, a-pinene, myrcene and terpinene in 1:1:1:1 ratio) against Staphylococcus aureus, Escherichia coli and Rhizoctonia solani. CK 
is control without terpenes. $5 \mathrm{~mm}$ sterilized white filter papers were placed in the centre or on the bottom of the petri dishes for antibacterial and antifungal assays respectively. $2 \mu$ of each VOC was added accordingly to the white filter papers. (B) Phylogenetic tree of characterized bacterial terpene cyclase proteins. The protein name indicates the bacterial species and the major terpene produced by these terpene cyclases. Sequences included are listed in Additional file 2: Table S13. (C) Ter91 terpene synthase with FPP. TIC $100 \%=1.76$ E5. Major sesquiterpene product at RT 15.17, identified as Germacrene D-4-ol by comparison of mass spectra to NIST14 spectral library

Biosynthesis of terpenes is mediated by terpene cyclases, and starts from polyprenyl pyrophosphate precursors. The precursor accepted by the cyclase determines which class of terpenes it produces: the $C_{10}$ precursor geranyl pyrophosphate (GPP) will lead to formation of monoterpenes, while $C_{15}$ precursorfarnesyl pyrophosphate (FPP) will lead to sesquiterpenes, and the $C_{20}$ precursor geranylgeranyl pyrophosphate (GGPP) will lead to di-terpenes, or to phytoene $\left(\mathrm{C}_{40}\right)$. In particular mono- and sesquiterpene synthases can occur in many cyclization patterns, leading to a huge diversity of molecules.

The genomes of the six Collimonas strains were screened for gene clusters possibly involved in terpene biosynthesis. All strains are carrying genes related to phytoene biosynthesis (Table S9). Only C. pratensis strains Ter91 and Ter291 harbored an additional terpene gene cluster comprising terpene synthases genes (Fig. 3C; CPter91_2617 and CPter291_2730). Terpene cyclases are widely distributed in bacteria, but mostly characterised in Streptomyces species (Cane \& Ikeda, 2012, Yamada et al., 2015). To date only one terpene cyclase from Proteobacteria has been functionally characterised, the 2-methylenebornane synthase from Pseudomonas fluorescens PfO-1 (Chou et al. 2011). CPter91_2617 and CPter291_2730 both encode a 330-amino acid protein that differs only in 2 amino acid residues (Fig. S5). When compared to other functionally characterized terpene cyclases, the Collimonas protein sequences showed maximally $23 \%$ aa-identity to any previously characterized bacterial terpene cyclase (Fig. 6B).

The product specificity of mono- and sesquiterpene cyclases cannot be predicted from their primary sequence. For biochemical characterization, CPter91_2617 and CPter291-2730 genes were expressed in E. coli, partially purified and tested in vitro using FPP, GPP or GGPP as substrates. When produced terpenes were analyzed by GC-MS, both Collimonas enzymes converted FPP to a mix of sesquiterpenes and sesquiterpene alcohols. The major peak was putatively identified as germacrene D-4-ol by comparison of the mass spectrum to the NIST 
2014 spectral library, and several minor sesquiterpene peaks, including $\delta$-cadinene (Fig. 6C). When GPP was applied as a substrate, production of two monoterpenes identified as $\beta$-pinene and $\beta$-linalool was observed (Fig. S6A). A small amount of product could be observed upon the incubation of GGPP as substrate, which was putatively identified as 13-epimanool (Fig. S6B). Thus we characterize CPter91_2617 and CPter291_2730 as mixed mono-, sesqui- and diterpene cyclases, with major product germacrene D-4-ol. The sesquiterpene products suggest they are functionally related to plant and fungal cadinene/cadinol and germacrene D-4-ol synthases, although sequence homology to these enzymes is low (Yoshikuni et al., 2006, Lauchli et al., 2014). One of the monoterpene products of CPter91_2617 and CPter291_2730, $\beta$-pinene, was also observed in the headspace of C. pratensis and showed to have antibacterial effect, suggesting a role of the Collimonas terpene cyclases in antimicrobial activity. Volatiles terpenes compound may have synergistic antimicrobial effect in combination with antibiotics. For example synergistic effect of terpenes and penicillin on multiresistant strains S. aureus and E. coli was reported (Gallucci et al., 2009). Beside antimicrobial activity the production of terpenes by Collimonas may point another important ecological role namely chemical communication. Since terpenes volatilize easily and can be produced by all kingdoms of life including plant, fungi and bacteria, we assume that terpenes may play significant roles for Collimonas long-distance inter-kingdom interactions and communications.

\section{Conclusions}

The comparative analysis of six completely sequenced Collimonas genomes representing three species revealed a high degree of genomic diversity between strains with a core genome representing $49.8 \%$ to $59.6 \%$ of the genome of all strains (Summarized in Fig. 3C). Although the genomes were largely syntenic, genomes rearrangements were observed both between and within the species indicating high genomic plasticity. All Collimonas genomes carry large numbers of Genomic Islands pointing at a complex history of gene recombination and horizontal transfer between bacterial relatives. Type two secretion systems were present in all genomes suggesting that it may be important trait for the mycophagy lifestyle of Collimonas.

Genomic analysis of secondary metabolism of Collimonas revealed that genes encoding for exoenzymes such as chitinase, peptidase, phospholipase are well conserved but that there is a high variability of genes encoding for other secondary 
metabolites such as collimomycin, lipopeptides, (PKS-)NRPS, and terpenes. Genes encoding the polyacetylenic compound collimomycin and lipopeptide tripropeptin $A$ are present only in $C$. fungivorans while the gene clusters encoding a putative new lipopeptide is present in both $C$. fungivorans and C. pratensis, but not $C$. arenae. Moreover, several unknown orphan (PKS-)NRPS gene clusters are present in the genome of $C$. fungivorans and $C$. pratensis.

Mutational and phenotypical analyses indicated that tripropeptin A and the designated new lipopeptide have antibacterial and antifungal (oomycetal) activities, respectively. The biochemical characterization of the terpene synthases genes revealed that Collimonas are able to produce a set of sesquiterpenes and/ or monoterpens that are considered to be mainly of plant origin. The in vitro assay of pure terpene compounds indicated their contributions to both antibacterial and antifungal activities.

Overall, our exploration of Collimonas genomes revealed that this bacterial group represent a valuable resource for the discovery of novel secondary metabolites and enzymes. The results gained based on comparative genome analysis here will be certainly helpful for designing future experimental studies that will lead to comprehensive understanding of the unique ecology of Collimonas species.

\section{Material and Methods}

\section{Strains and growth conditions}

All bacterial strains used in this study are listed in Table 1 and Table S10. Collimonas strains were cultured in 0.1 Tryptic Soy Broth (0.1 TSB) $(5 \mathrm{~g} / \mathrm{L} \mathrm{NaCl}, 1 \mathrm{~g} / \mathrm{L} \mathrm{KH2PO}$, 3g/L TSB, 20 gL-1 CMN-Boom Agar, pH=6.7) or King's B medium (20g/L proteose peptone, 1.5g/L MgSO $4^{\prime} 1.2 \mathrm{~g} / \mathrm{L} \mathrm{KH}_{2} \mathrm{PO}_{4^{\prime}} 10 \mathrm{~g} / \mathrm{L}$ glycerol, $15 \mathrm{~g} / \mathrm{L}$ agar). Escherichia coli strain DH5a was used as a host for the plasmids used for site-directed mutagenesis. E. coli strains were grown on Luria-Bertani (LB) plates (10 g/L NaCl, $10 \mathrm{~g} / \mathrm{L} \mathrm{Bacto}^{\mathrm{TM}}$ Tryptone, $5 \mathrm{~g} / \mathrm{L}$ Bacto ${ }^{\mathrm{TM}}$ Yeast extract, $20 \mathrm{~g} / \mathrm{L}$ Merck Agar) or in LB broth amended with the appropriate antibiotics. 


\section{Genomic DNA isolation}

Genomic DNA from each Collimonas strain was extracted from overnight grown cells using QIAamp® DNA Mini Kit and Qiagen ${ }^{\circledR}$ MagAttract ${ }^{\circledR}$ HMW kit and used for Illumina and PacBio RS II sequencing respectively.

\section{Genome sequencing}

Illumina paired-end sequences were obtained for C. arenae Ter10, C. arenae Ter282, C. pratensis Ter91, C. pratensis Ter291 and C. fungivorans Ter6 from BaseClear B.V. on the Illumina HiSeq2000 platform ( 2 × 51 bp paired-end reads, except strain Ter91 which was sequenced at $2 \times 100 \mathrm{bp}$ paired-end reads) and assembled using the Ray (Boisvert et al., 2010) assembler version 2.3.1 (Table S11).

PacBio RS II sequences were obtained from 5 SMRT cells, one for each strain. Sequences were filtered using SMRT Analysis server v2.2.0 with default settings. The RS_HGAP Assembly.3 (HGAP3) (Chin et al., 2013) protocol was used to assemble the filtered reads, the RS_AH_Scaffolding protocol was used if the initial assembly yielded more than one contig, followed by a final Quiver correction using the RS_Resequencing protocol (See Table S12).

The singular contigs were checked using a custom script and overlapping ends trimmed. The final circular contig for each chromosome was rearranged to start at the dnaA gene in the forward direction. There was no evidence for plasmids in the sequence data.

All Collimonas sequences including the previously published genome of Ter331 were annotated using the IGS annotation pipeline (Galens et al., 2011).

\section{Bioinformatic analysis}

Core, Pan and variable genome analysis:

Protein coding genes from the six Collimonas strains were clustered together with the protein coding genes of Burkholderia phytofirmans PSJN (NC_010681.1, NC_010676.1) and Pseudomonas protegens Pf-5 (NC_004129.6) using cd-hit (Huang et al., 2010)2010 with word length 3 (-n 3), global identity (-G 1) and a minimal alignment coverage of $60 \%$ for the shortest protein (-aS 0.6). Cd-hit clusters were parsed into an absence-presence matrix from which the core, pan and variable 
genomes were parsed using custom scripts. COG annotations were determined using kognitor (Snel et al., 2002). Core and pan evolution plot is generated based on (Tettelin et al., 2005). At each number of strains (n) out of strain set (s), s!/ $n ! *(s-n) !$ combinations are possible. The median number of specific, variable and core genes for all combinations are plotted as a function of $n$. Clusters containing one gene per strain were selected from the core cluster set of the Collimonas, Burkholderia and Pseudomonas strains were aligned with MAFFT and combined in one pseudoalignment. Redundant colums were removed and maximum likelihood phylogenetic trees were calculated with RAxML (Stamatakis, 2014).

The annotation of the previously-published genome of Ter331 was updated and manually curated as part of this study. Synteny analyses were performed using Progressive MAUVE (Darling et al., 2004)2004. Phylogenetic analyses on the whole genome was performed using Gegenees (Agren et al., 2012)2012, and 16S rDNA analysis was performed using MEGA6 (Felsenstein, 1985, Saitou \& Nei, 1987, Tamura et al., 2004, Tamura et al., 2013). Whole genome peptidases prediction was conducted by MEROPS (Rawlings et al., 2014)2014. Phospholipases were predicted by searching on the basis of the profile HMM using PFAM domains of phospholipase A1 (PF02253), phospholipase A2 (PF09056), phospholipase C (PF05506) and phospholipase D (PF00614). Secondary metabolite production clusters were examined using the antiSMASH program (Medema et al., 2011, Blin et al., 2013). The amino acid composition of products from NRPS sequences were predicted using NRPSpredictor 2 (Rottig et al., 2011). Genomic islands were identified using IslandViewer (Langille \& Brinkman, 2009, Dhillon et al., 2013) and phages elements and features were identified using PHAST (Zhou et al., 2011)2011. CRISPRs were identified based on CRISPRfinder (Grissa et al., 2007)2007. Whole genome analysis for type VI secretion system was conducted with SecRet6 (Li et al., 2015) and circular genome diagrams were visualized using Circos (Krzywinski et al., 2009)2009.

\section{Abbreviations}

PKS: polyketide synthase

NRPS: nonribosomal peptide synthetase

COG: Clusters of Orthologous Groups

GIs: Genomic Islands

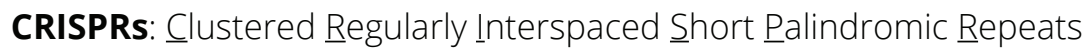




\section{Acknowledgements}

This research was supported in part by funds from Ecolink (260-45140) and by The Netherlands Organization for Scientific Research (NWO) VIDI personal grant to P.G (864.11.015). This publication is No.5983 of the Netherlands Institute of Ecology (NIOO-KNAW).

\section{Competing interests}

The authors declare no conflict of interest.

\section{Authors' contributions}

CS and PG designed the experiments. CS, RS and VJ performed the bioinformatics analysis. CS, DK, EJ, KC, AV conducted the experiments. CS drafted the manuscript. $P G, J B, K C, W B$ and JV revised the manuscript. All authors read and approved the final manuscript.

\section{Supplementary Materials}

\section{Additional detailed materials and methods}

\section{Quorum sensing assay}

Collimonas wild type strains, and indicator strain C. violaceum CV026 (50 $\mu \mathrm{g} / \mathrm{mL}$ kanamycin) (McClean et al., 1997), A. tumefaciens NT1 (Farrand et al., 2002) were grown in $10 \mathrm{ml} 0.1 \mathrm{TSB}$ broth overnight at $20^{\circ} \mathrm{C}$. The cells were washed twice with sterile $10 \mathrm{~mm}$ sodium phosphate buffer (1.361 $\mathrm{g} \mathrm{KH}_{2} \mathrm{PO}_{4}$ in $1 \mathrm{~L}$ milliQ, pH 6.5), adjusted to $1 \times 10^{8}$ cells $/ \mathrm{ml}, 5 \mu \mathrm{l}$ of cell suspension was spotted on $0.1 \mathrm{TSB}$ plates with indicator strain C. violaceum CV026 and 0.1 TSB plates (50 $\mu \mathrm{g} / \mathrm{mL}$ X-gal) with indicator strain A. tumefaciens NT1 and incubated at $20^{\circ} \mathrm{C}$ for $2-3$ days. Purple (C. violaceum CV026) and blue (A. tumefaciens NT1) colonies are indicating production of AHLs by the respective Collimonas strains.

\section{Siderophore detection assay}

ChromoAzurolS (CAS) and King's B (KB) medium were prepared based on previously description (Schwyn \& Neilands, 1987). KB medium consists of $20 \mathrm{~g}$ proteose 
peptone, $1.5 \mathrm{~g} \mathrm{MgSO}_{4^{\prime}} 1.2 \mathrm{~g} \mathrm{KH}_{2} \mathrm{PO}_{4^{\prime}}, 10 \mathrm{~g}$ glycerol, $15 \mathrm{~g}$ agar and water to $1 \mathrm{~L}$. Orange halos around the colonies on the blue CAS agar plates are indicative of siderophore production.

\section{Extracellular protease activity assay}

The cells from different strains were washed with sterile MilliQ water and set to a final density of $1 \times 10^{8}$ cells $/ \mathrm{mL}$ and $5 \mu \mathrm{L}$ of this bacterial suspension was spotted on Skim Milk Agar plates (SMA, 1 Liter: $15 \mathrm{~g}$ skim milk powder, $4 \mathrm{~g}$ blood agar base, $0.5 \mathrm{~g}$ yeast extract and $13.5 \mathrm{~g}$ agar) and incubated at $20^{\circ} \mathrm{C}$ for 4 days. Extracellular protease activity was quantified by measuring the diameter of the transparent halo surrounding the bacteria colony.

\section{Swimming motility}

Swimming motility assays of the bacterial strains were conducted according to the method described previously by de Bruijn \& Raaijmakers (2009). Swimming motility of the Collimonas strains were assessed on soft [0.3\% wt/vol] M9 medium. After autoclaving, the medium was cooled down in a water bath to $55^{\circ} \mathrm{C}$ and kept at $55^{\circ} \mathrm{C}$ for $1 \mathrm{~h}$. Twenty $\mathrm{ml}$ of the medium was pipetted into a 9 -cm-diameter petri dish, and the plates were kept for $24 \mathrm{~h}$ at room temperature $\left(20^{\circ} \mathrm{C}\right)$ prior to the swimming assay. For all swimming assays, the same conditions (agar temperature \& volume, time period of storage of the poured plates) were kept constant to maximize reproducibility. Overnight cultures of Collimonas strains were washed three times with $0.9 \% \mathrm{NaCl}$, and $5 \mu \mathrm{L}$ of the washed cell suspension $\left(1 \times 10^{8}\right.$ cells $\left./ \mathrm{ml}\right)$ was spot inoculated in the centre of the soft agar plate and incubated for 3 days at $20^{\circ} \mathrm{C}$.

\section{Mutagenesis of new lipopeptide and tripropeptin A genes}

Site-directed mutagenesis of the new lipopeptide and tripropeptin A was performed with the pEX18Tc suicide vector as described by Choi and Schweizer (2005). For each mutant construct, a 5' fragment, a Gm cassette and a 3' fragment were synthesized for the target gene in Baseclear, Leiden, the Netherlands (www.baseclear.com), and constructed to vector pEX18Tc. The synthesized sequences are given in the end of this file. The mutant constructs pEX18Tc-NLP and PEX18Tc-trpA were were subsequently electroporated into C. fungivorans Ter331. Electrocompetent cells were obtained according to the method of Choi et al. (2006) and the electroporation was performed at $2.4 \mathrm{kV}$ and $200 \mu \mathrm{F}$. After incubation in SOC medium (2\% Bacto 
tryptone [Difco], 0.5\% Bacto yeast extract [Difco], $10 \mathrm{mM} \mathrm{NaCl}, 2.5 \mathrm{mM} \mathrm{KCl}, 10 \mathrm{mM}$ $\mathrm{MgCl} 2,10 \mathrm{mM} \mathrm{MgSO} 4,20 \mathrm{mM}$ glucose $[\mathrm{pH} 7])$ for $2 \mathrm{~h}$ at $25^{\circ} \mathrm{C}$, the cells were plated on KB supplemented with gentamicin $(40 \mu \mathrm{g} / \mathrm{ml})$. The obtained single crossover colonies were grown in LB overnight at $25^{\circ} \mathrm{C}$ and plated on LB supplemented $5 \%$ sucrose and gentamicin $(40 \mu \mathrm{g} / \mathrm{ml})$ to accomplish the double crossover. The plates were incubated at $25^{\circ} \mathrm{C}$ for at least $48 \mathrm{~h}$, and colonies were re-streaked on $\mathrm{KB}$ supplemented with gentamicin $(40 \mu \mathrm{g} / \mathrm{ml})$ and tetracycline $(25 \mu \mathrm{g} / \mathrm{ml})$. Colonies that grew on KB with gentamicin, but not on KB with tetracycline, were selected and subjected to colony PCR to confirm genes mutagenesis.

\section{Phylogenetic analysis of terpene synthases}

The deducted protein sequence of terpene synthases CPter91_2617 and CPter291_2730 was compared to previously characterized terpene synthases from genera Streptomyces, Saccharopolyspora, Saccharothrix, Streptosporangium and Pseudomonas. A full list of sequences included for phylogenetic analysis is given in Supplemental table S14. Multiple protein sequence alignments and bootstrap $\mathrm{N}$-J trees were generated by the CLC Workbench software, with a 100 replicates of bootstrap analysis.

\section{Heterologous expression of terpene synthases in E. coli and enzyme activity assays}

PCR-generated DNA encompassing the complete coding sequence of terpene synthases genes of CPter91_2617 and CPter291_2730 were inserted into the cloning site of the expression vector pACYCDuet-1 $\left(\mathrm{Cm}^{\mathrm{R}}\right)$. The constructs were introduced into E. coli BL21 DE3 and protein expression and enzyme activity assays were performed as described in Jongedijk et al. (2015). $5 \mu 10 \mathrm{~mm}$ GPP, FPP or GGPP were added as substrates to the assay mix. The mix was immediately covered with an overlay of $1 \mathrm{ml}$ pentane and incubated at $30^{\circ} \mathrm{C}$ for $1 \mathrm{~h}$ under gentle agitation. The tubes were vortexed well and centrifuged for $5 \mathrm{~min}$ at $3400 \mathrm{rpm}$. The pentane phase was collected, dried over anhydrous $\mathrm{Na}_{2} \mathrm{SO}_{4}$ and injected into a 7890A gas chromatograph (Agilent) equipped with a mass selective detector (Model 5975C, Agilent), scanning in the range 45-450 m/z. Splitless injection of $1 \mu \mathrm{l}$ sample was performed at $250^{\circ} \mathrm{C}$ on a Zebron ZB-5MS column $(30 \mathrm{~m} \times 0.25 \mathrm{~mm}, 0.25$ $\mu \mathrm{m}$ thickness; Phenomenex) at a helium flow rate of $1 \mathrm{ml} / \mathrm{min}$. The temperature programme was $2.25 \mathrm{~min}$ at $45^{\circ} \mathrm{C}$, then the temperature was increased at the 
rate of $40^{\circ} \mathrm{C} / \mathrm{min}$ to $300^{\circ} \mathrm{C}$, followed by 3 min at $300^{\circ} \mathrm{C}$. Standard of $\beta$-pinene was purchased from Acros.

\section{Antimicrobial activities assay}

The antifungal and anti-oomycete activities of the Collimonas strains and mutants were tested as follows: Collimonas strains and mutant strains were grown in $5 \mathrm{ml}$ KB broth overnight at $25^{\circ} \mathrm{C}$. Strip $50 \mu \mathrm{l}$ bacterial suspension $\left(1 \times 10^{8} \mathrm{cells} / \mathrm{ml}\right)$ in the middle of a $0.1 \mathrm{TSB}$ plate. After three days of incubation at $20^{\circ} \mathrm{C}$, a mycelial plug of 4-mm diameter of each fungal or oomycete pathogen was placed in the edge of the $0.1 \mathrm{TSB}$ plate and incubated at $20^{\circ} \mathrm{C}$. Migration diameters of the fungus or oomycete were measured for 6-7 days depending on the pathogen's growth rate.

To test the antibacterial activity of Collimonas strains or constructed mutants, $10 \mu \mathrm{l}$ of cell suspension was spotted on 0.1 TSB plates and incubated at $20^{\circ} \mathrm{C}$ for 3 days. Subsequently, overnight cultures of S. aureus was washed twice with sterile milliQ water, and cell suspensions $\left(2 \times 10^{6}\right.$ cells $\left./ \mathrm{ml}\right)$ were overlaid onto the Collimonas/mutant inoculated agar surface and incubated at $30^{\circ} \mathrm{C}$ overnight. The antibacterial activity was observed by the formation of visible zones of inhibition of the bacterial pathogens.

Additional file1: Fig. S1 / Phylogenetic tree based on 16S rDNA depicting the relationships of sequenced strains of Collimonas. Fig. S2 / Synteny of the six Collimonas genomes. Pairwise alignments of genomes were generated using Mauve (A) C. fungivorans (B) C. pratensis (C) C. arenae and (D) The three species together. Colored outlined blocks surround the regions of the genomic sequence that aligned to another genome. The colored bars inside the blocks are related to the level of sequence similarities. The analysis showed that the highest number of rearrangements was evident between all the three species.Fig. S3 / (A) Conserved gene clusters for type II (T2SSa/T2SSb), III (T3SSa/T3SSb) and VI (T6SS) secretion systems identified in Collimonas strains. Quorum sensing assays of the Collimonas strains. Quorum sensing activity of Collimonas strains Ter331, Ter6, Ter91, Ter291, Ter10 and Ter282 with indicator strain (B) C. violaceum CV026 and (C) A. tumefaciens NT1 (outer colonies). A purple (C. violaceum CV026) or blue (A. tumefaciens NT1) pigment produced by the indicator strains is indicative of quorum sensing activity of the tested strains. Fig. S4 / Organization of the orphan gene clusters and predicted amino acid compositions.Fig. S5 I Amino acid alignment of Collimonas terpene synthases CPter91_2617 and CPter291_2730 with previously characterised bacterial terpene synthases. The Collimonas terpene synthases were aligned with the Streptomyces exfoliatus pentalenene synthase (Se_pentalenene), S. coelicolor geosmin synthase (Sc_geosmin, 336 amino acids of the N-terminus), Streptosporangium roseum epi-cubenol synthase (Sr_epicubenol), S. avermitilis avermitilol synthase (Sa_avermitilol), S. clavuligerus 1,8-cineole synthase (Scl_cineole) and Pseudomonas fluorescens 2-methylenebornane synthase (Pf methylenebornane). The characteristic terpene synthase divalent metal-binding motifs, namely the acidic amino acid-rich motif and the NSE triad, are boxed. Fig. S6 / GC-MS chromatograms of Ter91 
terpene cyclase incubated with different substrates, and mass spectra of major products. The GC-MS chromatograms of Ter291 were identical to Ter91 (data not shown). Empty vector chromatograms shows products from a control enzyme extract (PACYC-duet-1). (A) Ter91 terpene synthase with GPP. TIC 100\% $=1.19 \mathrm{E} 4$. Major monoterpene product at RT 6.79, identified as Beta-pinene by authentic standard. (B) Ter91 terpene synthase with GGPP. TIC 100\% = 2.12E4. Major diterpene product at RT 20.24, identified as 13-epimanool comparison of mass spectra to the NIST8 library (available online with this article).

Additional file 2: Table S1 / Gene locus of the flagellar biosynthetic gene cluster of the Collimonas strains. Table $\mathbf{S} \mathbf{I}$ Gene locus of the secretion systems of the Collimonas strains. Table S3 / Genomic distribution of signal transduction proteins in six Collimonas genomes. Table S4 I Presence of quorum-sensing proteins in six Collimonas genomes. Table $\mathbf{5 5}$ / Gene locus of the collimomycin biosynthetic gene cluster of the Collimonas strains. Table $\mathbf{6} \mathbf{6}$ / Gene locus of the chitinase biosynthetic gene clusters of the Collimonas strains. Table S7 I Gene locus of the phopholipase genes of the Collimonas strains. Table $\mathbf{8 8}$ / Antimicrobial activities of the Collimonas strains. Table $\mathbf{5 9}$ / Genes encoding secondary metabolites and secretion systems in Collimonas strains. Table S10 / Strains and mutants used in this study. Table S11 / Summary of Illumina paired-end sequences assembly data. Table S12 / Summary of PacBio sequences assembly data. Table S13 / Sequences of characterized bacterial terpene synthases used in the phylogenetic analysis. (available online with this article).

Additional file 3: The IGS annotation of Collimonas fungivorans Ter331 genome. (available online with this article). 


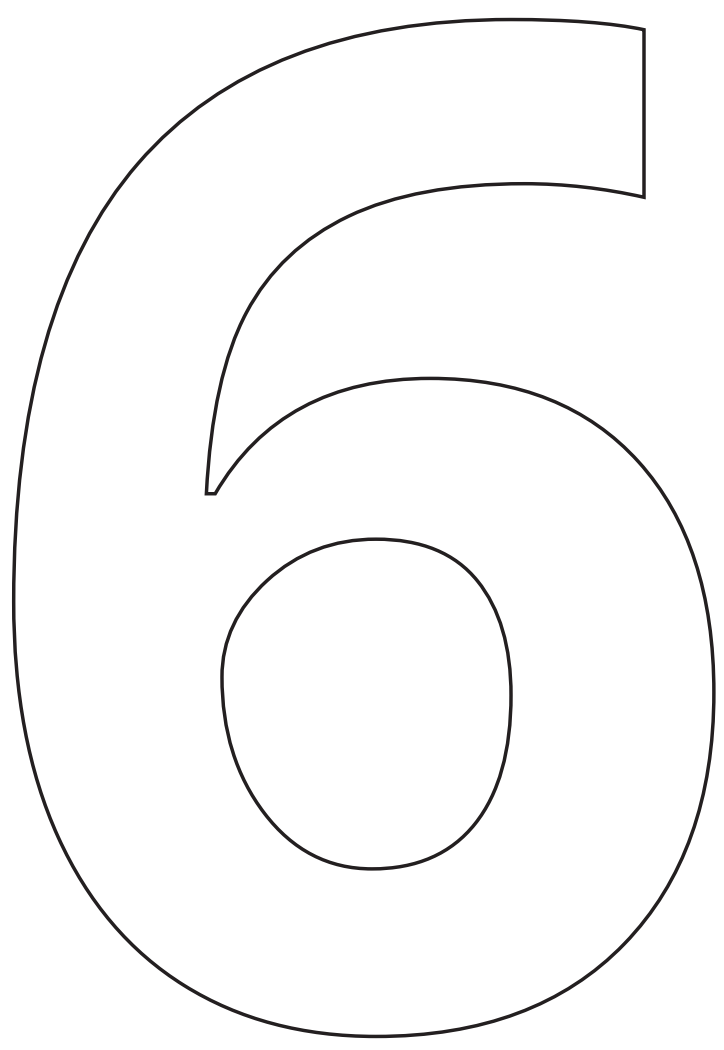

\section{Deciphering the Fusarium culmorum genome: from genes to products}

Ruth Schmidt, Mikael B. Durling, Victor de Jager, Riya C. Menezes, Erik Nordkvist, Aleš Svatoš, Mukesh Dubey, Lukas Lauterbach, Jeroen Dickschat, Magnus Karlsson and Paolina Garbeva 



\begin{abstract}
Fusarium culmorum is a one of the most important fungal plant pathogens that causes diseases on a wide diversity of cereal and non-cereal crops. We report herein for the first time the genome sequence of $F$. culmorum $P V$ and its associated secondary metabolome that plays a role in the interaction with other microorganisms and contributes to its plant pathogenicity. The genome revealed the presence of two terpenes synthases, trichodiene and longiborneol that generate an array of volatile terpenes. Furthermore, we identified two gene clusters, DON and ZEN that encode for the production of mycotoxins. Linking the production of mycotoxins with in vitro bioassays, we found a high pathogenicity of F. culmorum on maize, barley and wheat. Using a combination of LESA and UPLC/MS identified several other compounds important for F. culmorum behaviour and lifestyle. This research set the basis for future studies in microbe-plant interactions and for finding solutions for sustainable agricultural practices.
\end{abstract}

Keywords: Genome; Fusarium culmorum; Pathogenicity; Volatile organic compounds; Terpenes 


\section{Introduction}

Fungi produce a plethora of structurally diverse secondary metabolites. Due to their broad range of biological activities, secondary metabolites can provide an advantage to the producing organism under different environmental conditions (Demain \& Fang, 2000, Keller et al., 2005, Keller, 2015). Many fungi release both volatile and polar water-soluble compounds (Keller et al., 2005, Morath et al., 2012, Keller, 2015, Dickschat, 2017), some of which exhibit antagonistic bioactivity towards competing organisms in the same habitat while others function as signalling chemicals.

Volatile organic compounds (VOCs) are recently discussed to not only act in the direct surrounding of their producer, but also in long-distance microbial interactions (Effmert et al., 2012, Schmidt et al., 2015). Especially, terpenes were shown to play important roles in the long-range communication between microorganisms (Schmidt et al., 2017, Schulz-Bohm et al., 2017). Arguably, terpenes represent the largest and structurally most diverse class of natural products with over 55,000 members so far described, including monoterpenes, sesquiterpenes, and diterpenes. These molecules are generated via terpene cyclases, a highly sophisticated class of enzymes that turns simple linear and achiral precursor molecules with high enantioselectivity into usually polycyclic terpene hydrocarbons or alcohols with often multiple stereogenic centres (Degenhardt et al., 2009, Quin et al., 2014, Dickschat, 2016). The initial products can be further converted into highly functionalised and bioactive molecules such as mycotoxins by oxidations, acylations and other modifications. In the past two decades, several fungal terpene cyclases and their related products have been characterised, including the sesquiterpene cyclases for (-)-(1R,4R,5S)-guaia-6,10(14)-diene, (+)-eremophilene, (+)-koraiol and (-)-a-acorenol from Fusarium fujikuroi (Brock et al., 2013, Burkhardt et al., 2016), longiborneol from F. graminearum (McCormick et al., 2010), trichodiene from F. sporotrichioides (Hohn \& Beremand, 1989) and aristolochene from Penicillium roqueforti (Proctor \& Hohn, 1993)and bacterial expression of a sesquiterpenoid biosynthetic gene (Ari1. Although the principal processes of terpene biosynthesis are well understood, it remains a challenging task to predict the structure of a terpene just from the amino acid sequence of its corresponding synthase. With the advances in sequencing technology, whole genome sequences have become available for identification and mining of unexplored secondary metabolite genes.

The genus Fusarium represents an important group of fungal plant 
pathogens, causing various diseases on a broad spectrum of crops worldwide, including cereal and non-cereal crops (Scherm et al., 2013, King et al., 2015). For instance, F. culmorum causes foot and root rot (FRR) and fusarium head blight (FHB) on different small-grain cereals, in particular wheat and barley. Of equal concern is the health hazard posed to humans and livestock by F. culmorum mycotoxins, such as type B trichothecenes, zearalenone and fusarins of which the trichothecenes that derive from the sesquiterpene hydrocarbon trichodiene are considered the most bioactive compounds (Scherm et al., 2013). These mycotoxins are able to inhibit eukaryotic protein biosynthesis, cause toxicoses in humans or animals consuming contaminated food, have been reported to induce apoptosis, and play an important role as virulence factors (Scherm et al., 2013). However, despite the ecological and agricultural importance of $F$. culmorum its genome remains unknown. Our previous research revealed that $F$. culmorum emits a specific blend of VOCs including various terpenes (Schmidt et al., 2015). Furthermore, we observed that F. culmorum VOCs play an important role in the long-distance interaction with bacteria by inducing both motility and production of secondary metabolites.

The aim of this study was to sequence the genome of F. culmorum and to explore and identify genes associated with secondary metabolite production that potentially play important roles in fungal pathogenicity and fungal-bacterial interactions. Furthermore, we aimed to correlate the genomic data with metabolomic analyses of water-soluble and volatile compounds.

\section{Materials and Methods}

\section{Fungal strains and growth conditions}

The F. culmorum isolate PV, originally isolated from a sandy dune soil in The Netherlands (De Boer et al., 1998), was used in this study. The isolate was grown on King's B agar, supplemented with $50 \mathrm{mg} / \mathrm{mL}$ rifampicin, kanamycin and ampicillin and subsequently transferred to Oatmeal agar OA (24 g/L Difco), supplemented with $50 \mathrm{mg} / \mathrm{mL}$ rifampicin, kanamycin and ampicillin. The spores were washed with $50 \mathrm{mg} / \mathrm{mL}$ rifampicin, kanamycin and ampicillin, collected over glass wool with sterile deionized water and stored at $-80^{\circ} \mathrm{C}$. 


\section{Bioassay with F. culmorum on wheat and barley}

Pathogenicity of F. culmorum was tested on wheat and barley in an in vivo bioassay using a sand seedling test. Per treatment, five biological replicates with 10 plants per replicate were used following the procedure as described previously (Knudsen et al., 1995, Dubey et al., 2014). The seeds were surface sterilised and sown in a 1- to 2 -cm hole, 1 seed in each hole, in moistened, autoclaved sand in individual plastic pots $(5 \times 5 \times 5 \mathrm{~cm})$. Pathogen inoculation was carried out by placing a $5-\mathrm{mm}$ PDA plug containing F. culmorum mycelia close to the seed. PDA plugs without F. culmorum were used as control. After inoculations, the holes were filled with moist sand. Pots were kept in a growth chamber with a photoperiod of $12 \mathrm{~h}$ light, with $150 \mu \mathrm{Mol} \mathrm{m} \mathrm{m}^{-1}$ light intensity, and $12 \mathrm{~h}$ dark, 70\% \pm 5 relative humidity, and $20^{\circ} \mathrm{C} \pm 1$ temperature. The shoot length was measured every 5 days. Seedlings were harvested 3 weeks post inoculation and root length and fresh biomass were determined. Disease symptoms were scored on a 0 to 4 scale as described previously (Knudsen et al., 1995): 0 = healthy plants with no symptoms, 1 = slightly brown roots and coleoptiles, 2 = moderately brown roots and coleoptiles, 3 = severely brown roots and coleoptiles, and $4=$ dead plants. Pairwise comparisons were made using the Tukey-Kramer method at the $95 \%$ significance level $(P \leq$ 0.05). Results are shown as mean \pm standard deviation (S.D.).

\section{Fungal DNA extraction and genome sequencing}

Fungal plugs were grown on 0.5 strength PDA plates at $20 \mathrm{C}$ for 5 days. The mycelium was removed from the surface of the agar with a sterile scalpel and transferred to $200 \mathrm{~mL}$ 1.75\% malt extract $+0.25 \%$ peptone supplemented with 50 $\mathrm{mg} / \mathrm{mL}$ rifampicin, kanamycin and ampicillin and incubated for 10 days at $20^{\circ} \mathrm{C}, 120$ rpm. The liquid was removed by vacuum filtration and the mycelium was freezedried and stored at -20 C. $500 \mathrm{mg}$ of freeze-dried mycelium was ground to a fine powder in a sterile mortar in liquid nitrogen using a pre-cooled pestle. The ground mycelium was immediately transferred to $10 \mathrm{~mL}$ G2 buffer of the genomic DNA extraction kit (Qiagen), $10 \mu \mathrm{l}$ RNase $\mathrm{A}(10 \mathrm{mg} / \mathrm{mL})$ and $100 \mu$ l proteinase $\mathrm{K}(20 \mathrm{mg} /$ $\mathrm{mL}$ ) and incubated at $50^{\circ} \mathrm{C}$ for one hour and mixed by inverting the tube several times. The suspension was centrifuged twice at 5,000 rpm for 10 min at $4^{\circ} \mathrm{C}$ and the supernatant was transferred to $4 \mathrm{ml}$ QBT buffer (Qiagen) equilibrated Genomic-tip 100/G (Qiagen) and fixed in a $50 \mathrm{~mL}$ Falcon tube. The next steps were performed according to the midi-prep instructions of the Genomic-tip Protocol as part of the 
Genomic DNA Handbook (Qiagen). After isopropanol precipitation, the DNA was harvested using a glass rod, washed with 70\% ethanol, dried and dissolved in 200 $\mu \mathrm{L}$ Nuclease-free water and stored at $-20 \mathrm{C}$. The quality and quantity of the DNA was checked on a 0.8\% agarose gel with the O'GeneRuler $1 \mathrm{~kb}$ DNA Ladder and the 23 kb DNA Molecular Weight Marker II (Sigma-Aldrich). High-quality genomic DNA was then submitted to the Institute of Genome Sciences (Baltimore, Maryland, USA) for generation of a 15-30 kb fragment library, followed by and PacBio RS II sequencing. The genome was deposited at NCBI as Bioproject PRJNA375977.

\section{Genome assembly and annotation}

F. culmorum reads were extracted and assembled with the SMRT Portal software (smrtanalysis_2.3.0.140936.p4.150482) using the HGAP3 (Chin et al., 2013) assembly protocol with an estimated assembly length of $39 \mathrm{Mb}$ and default settings. The resulting contigs were scaffolded using PBJelly v15.8.24 (publicly available at https:// sourceforge.net/projects/pb-jelly/) with the same PacBio reads as in the HGAP3 assembly. The scaffolds of the PBJelly analysis were used as reference for 2 rounds of Quiver polishing using the BAM_RS_resequencing_Beta.1 protocol of the SMRT Portal software. The resulting polished contigs were annotated using Maker 2.31 with the available Fusarium gene models from Augustus and Ensembl predicted proteins of F. graminearum str. PH-1 and F. culmorum UK99. The resulting polished contigs were matched against the reference genome of $F$. graminearum str. $\mathrm{PH}-1$ using CONTIguator (Galardini et al., 2011) to get an indication of which contigs belong to which chromosome. To filter potential bacterial sequences out of the assembly, blobology (publicly available at https://github.com/blaxterlab/blobology) was run on the unmapped contigs. Protein-coding genes in the F. culmorum genome were annotated using MAKER 2 (Holt \& Yandell, 2011). We configured MAKER 2 to use SNAP (Korf, 2004), Augustus (Stanke \& Waack, 2003), and GenemarkES (TerHovhannisyan et al., 2008)2008 for ab initio gene calls. The Core Eukaryotic Genes Mapping Approach (CEGMA) program v2.4.01031218 (Parra et al., 2007) was used to detect the presence of 248 widely conserved eukaryotic core genes (CEG).

\section{Phylogenetic Analysis}

A custom pipeline was used for construction of a phylogenomic tree by using whole predicted proteome sequences. Six species were included for the topology construction, including F. graminearum (isolate $\mathrm{PH}-1$ ), F. oxysporum forma specialis 
(f. sp.) lycopersici (isolate FOL 4287), F. solani (isolate 77-13-4), F. verticillioides (isolate 7600), and F. fujikuroi (isolate IMI 58289), and Clonostachys rosea (isolate IK726) as outgroup. Except for C. rosea, the predicted proteome sequences of the included species were obtained from the JGI Mycocosm portal (Grigoriev et al., 2014)2014. The C. rosea proteome was predicted using the funannotate pipeline (publicly available at https://github.com/nextgenusfs/funannotate). The predicted proteomes were clustered using cd-hit and clusters were matched with the BUSCO fungal dataset (Simão et al., 2015). Predicted proteins occurring once in all six species were selected and aligned. A consensus tree was build by concatenating the variation from the selected core proteins using RaxML (Stamatakis, 2014). Phylogenetic trees were visualised using FigTree (publicly available at http://tree.bio.ed.ac.uk/software/ figtree/).

\section{In silico identification of secondary metabolite gene clusters}

For identification of secondary metabolite gene clusters antiSMASH 2.0 (Blin et al., 2013) was used. BLAST homology searches were performed with mycotoxin genes described in the closely related strain F. graminearum.

\section{Terpene production and phylogeny of terpene cyclases}

An agar plate culture of $F$. culmorum was inoculated with small pieces of an agar plate preculture and grown at $28^{\circ} \mathrm{C}$ for 3 days. The emitted volatile mono- and sesquiterpenes were trapped on charcoal filters by use of a closed-loop stripping apparatus for $24 \mathrm{~h}$, followed by extraction of the traps with dichloromethane. The obtained extracts were analysed by GC/MS and compounds were identified by comparison of the recorded mass spectra to library spectra, and of retention indices determined from a homologous series of $\mathrm{n}$-alkanes with published reference data. Positive compound identification was assumed for mass spectral matches >900/1000 and measured retention indices deviating no more than 10 units from the published value.

The terpene cyclases encoded in the genome of $F$. culmorum were identified by a sequence similarity search using BLAST with the trichodiene from $F$. sporotrichioides (Hohn \& Beremand, 1989) as a probe, resulting in two candidates with 90\% (F. culmorum trichodiene synthase) and 24\% (F. culmorum longiborneol synthase) sequence identity. Both putative terpene cyclases were inspected for 
the presence of the highly conserved motifs required for functionality, including the aspartate-rich motif (DDXXD) (Cane et al., 1996)1996, the NSE triad (ND(L,I,V) XSXXXE), the pyrophosphate sensor (highly conserved arginine ca. 46 residues upstream of the NSE triad) (Baer et al., 2014), and the RY dimer near the C-terminus. A phylogenetic tree containing the two terpene cyclases from F. culmorum and 2501 other terpene cyclase homologs that were all checked for the presence of the highly conserved motifs was constructed using the tree builder of Geneious 7.0.6 (Biomatters).

\section{Mycotoxin production}

$20 \mathrm{~g}$ of maize or wheat were weighted into a $150 \mathrm{ml}$ Erlenmeyer flask and $15 \mathrm{ml}$ distilled water was added. The bottle was autoclaved for $1 \mathrm{~h}$ at $120^{\circ} \mathrm{C}$. After cooling, three agar plugs of $6 \mathrm{~mm}$ in diameter were inoculated onto the substrate from 1-week-old F. culmorum cultures. Sterilized maize and wheat grains without $F$. culmorum served as control. After the incubation period, the grain cultures were freeze dried and finely ground to pass a 1.0-mm sieve by an impact crusher (Retsch ZM 100, Nino Lab, Stockholm, Sweden). The samples were analysed by LC-MS/MS according to Tevell Åberg et al. (2013).

\section{Analysis of secondary metabolites using UPLC-MS/MS}

F. culmorum agar plugs were used to inoculate 0.5 strength PDA plates and incubated at $28^{\circ} \mathrm{C}$ for 4 days. The mycelium was scraped off the agar and homogenized with liquid nitrogen in a glass vial, $2.5 \mathrm{ml}$ ice-cold solvent of methanol and water (1:2) were added and the mixture was ultrasonicated for $30 \mathrm{~min}$. The sample was then centrifuged to separate the cell debris. The clear supernatant was collected. To the pellet, $2 \mathrm{ml}$ of water and chloroform (1:1) was added. The sample was ultrasonicated and spun. Finally, the two liquid phases were separated. The polar and non-polar fractions were analyzed separately with UPLC-MS. Liquid extraction surface analysis (LESA) was performed using a Triversa Nanomate chip based electrospray ionization system (Advion, Ithaca, NY, USA) coupled to a LTQ Orbitrap - XL (Thermo Fisher Scientific, Bremen, Germany) mass spectrometer operated in positive and negative ion mode. Ultra-performance liquid chromatography-mass spectrometry (UPLC-MS) analyses of F. culmorum extracts were performed on a Q-Exactive Plus Hybrid Quadrupole-Orbitrap Mass Spectrometer (Thermo Fisher Scientific, Bremen, Germany) equipped with a Ultimate 3000 series RSLC (Dionex, Sunnyvale, 
CA, USA) chromatograph. Chromatographic separation was achieved on an Acclaim C18 column $(150 \times 2.1 \mathrm{~mm}, 2.2 \mu \mathrm{m}$ particles with $120 \AA$ pore diameter, Dionex, Sunnyvale, CA, USA) with a flow rate of $300 \mu \mathrm{m} \mathrm{min}^{-1}$ in a binary solvent system of water (Solvent A) and acetonitrile (Solvent B), both containing $0.1 \%(v / V)$ formic acid. $5 \mu$ each of extracts were loaded onto the column and eluted by using a gradient as follows: linear increase from 0\% B to 100\% B within 15 min - 100\% B constant for 5 min - equilibration time at 0\% B for 5 min. The mass spectrometer was operated in positive and negative ionization modes using Heated-Electrospray Ionization (H-ESI) in the mass range of m/z 100 to 1500 using 70,000 m/Am resolving power in the Orbitrap mass analyzer. $\mathrm{H}$-ESI source parameters were set to $4 \mathrm{kV}$ for spray voltage, $35 \mathrm{~V}$ for transfer capillary voltage at a capillary temperature $300{ }^{\circ} \mathrm{C}$. Data was evaluated and interpreted using Xcalibur v.3.0.63 software (Thermo Fisher Scientific, Waltham, MA, USA). Fragmentation was achieved with data dependent acquisition using a resolution of $70,000 \mathrm{~m} / \Delta \mathrm{m}$ and stepped normalized collision energy of 10,30 and 60 .

\section{Results and Discussion}

\section{Pathogenicity of $F$. culmorum on wheat and barley}

First, we tested the pathogenicity of $F$. culmorum on barley and wheat. Our result showed a significant $(P \leq 0.05)$ decrease in the shoot length of barley and wheat seedlings when infected with F. culmorum over a period of two weeks as compared to the control (Fig. 1A). Disease symptoms were high with severely brown roots and coleoptiles in both barley and wheat (Fig. 1B). Moreover, root length and fresh weight were significantly $(P \leq 0.05)$ reduced in both barley and wheat when infected with F. culmorum as compared to the control (Fig. 1C and D). These results show the high prevalence of F. culmorum as a pathogen in both crops.

The lack of full F. culmorum genome has limited the functional analysis of genes to few examples (Ward etal., 2002, Scherm etal.,2011). To link the pathogenicity to secondary metabolites produced by F. culmorum, such as mycotoxins, the next step was to sequence and investigate its genome. 

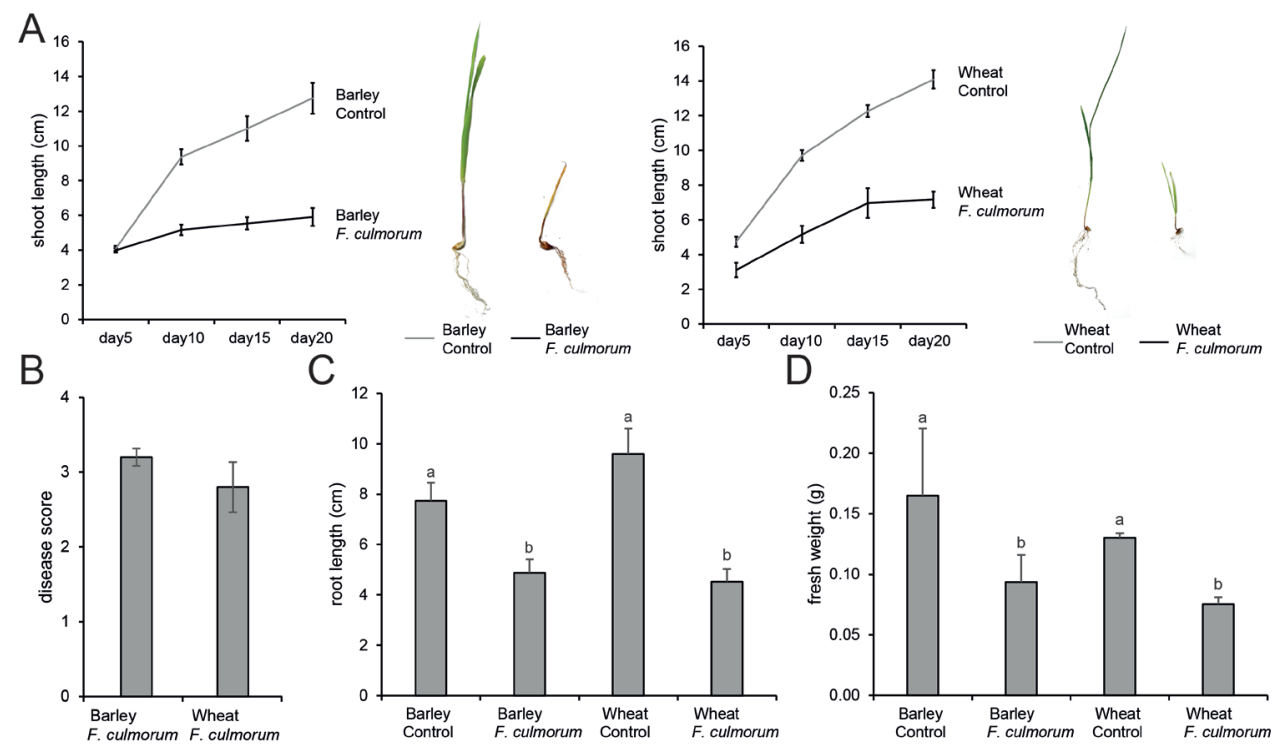

D

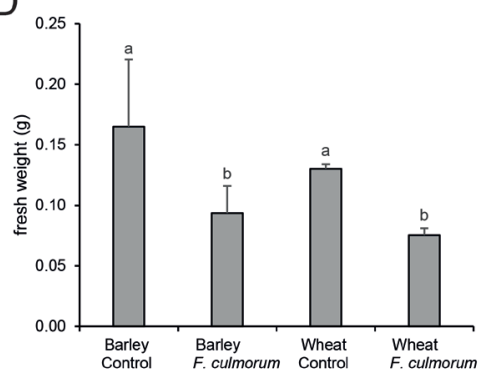

Fig. 1 I In vivo pathogenicity test of $F$. culmorum on wheat and barley. Seeds were planted in moist sand together with a F. culmorum agar plug. The shoot length was measured every 5 days (A). Seedlings were harvested 3 weeks post inoculation and disease symptoms were scored on a 0 to 4 scale (B) (no disease symptoms were observed for the controls), and root length (C) and fresh biomass (D) were determined. The experiment was performed in five biological replicates with 10 plants in each replicate. Error bars represent standard deviation based on five biological replicates. Different letters indicate statistically significant differences $(P \leq 0.05)$ within experiments based on the Tukey-Kramer test.

\section{General features of $F$. culmorum}

The F. culmorum genome was deep sequenced using 5 PacBio SMRT cells to at least $115 \times$ coverage and assembled with HGAP3. The total assembly length was 38.24 Mb spread across 37 scaffolds with an $\mathrm{N}_{50}$ read length of $5215.4 \mathrm{~kb}$ (Table 1). 237 of 248 CEGMA genes are complete in our gene list (95.6\%), and an additional 6 genes are represented as fragments (Table S1). The mapping of F. culmorum genome resulted in mapped contigs to 4 chromosomes, 1 mitochondrial chromosome and 12 unmapped contigs (Table S2). A total of 12,979 gene models coding for proteins were annotated using MAKER 2. All gene models were functionally annotated using InterProScan5 to assign Gene Ontology (GO) terms (Ashburner et al., 2000) and Pfam domains (Table 1, Fig. 2). Pfam domains were found in 8,688 gene models, whereas $\mathrm{GO}$ terms were assigned to 6,917 genes. 
Table 1 / General features of the Fusarium culmorum genome: assembly and annotations

\begin{tabular}{ll}
\hline Genome size (Mb) & 38.24 \\
Number of scaffolds & 37 \\
Gap length & 0 \\
G+C content (\%) & 47 \\
Scaffold N50 (kb) & 5215.4 \\
Gene count & 12,979 \\
Total coding length (Mb) & 18.5 \\
Genes with EST support & 12,487 \\
Average/median transcript length (bp) & $1,598.04 / 1,324$ \\
Average/median exon count & $3.01 / 3$ \\
\hline
\end{tabular}
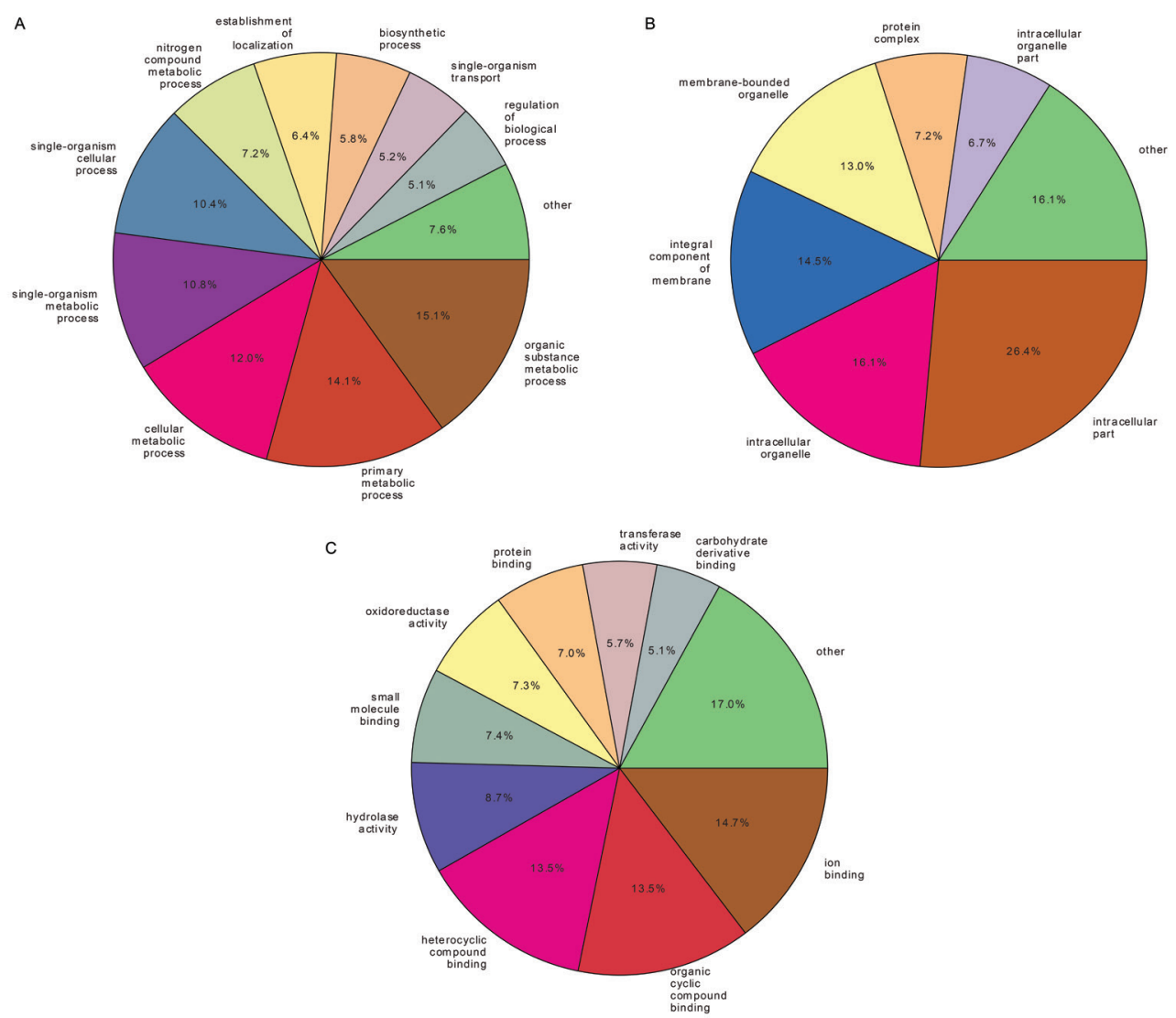

Fig. 2 / Functional annotations of F. culmorum genes. GO terms of the three ontologies biological process (A), cellular component (B) and molecular function (C) were assigned using InterProScan. 


\section{Phylogenetic placement of $F$. culmorum}

In order to determine the phylogenetic relationship between F. culmorum and other sequenced Fusarium species, a whole-proteome analysis was conducted. The phylogenetic analysis showed that F. culmorum clustered together with $F$. graminearum (Fig. 3). F. fujikuroi and F. oxysporum f. sp. lycopersici clustered as sister taxa with 100\% bootstrap support. F. solani clustered with the outgroup C. rosea with 100\% bootstrap support.

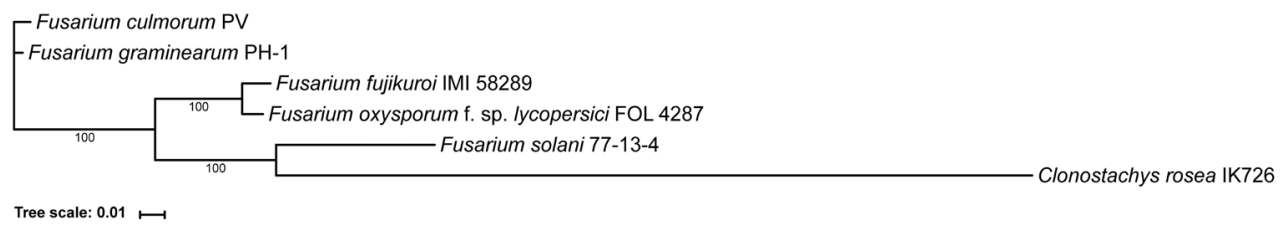

Fig. 3 / Phylogenetic placement of F. culmorum. The phylogenomic tree was constructed using whole predicted proteome sequences. Six species were included for the topology construction, including F. graminearum, F. oxysporum f. sp. lycopersici, F. solani, F. verticillioides, and F. fujikuroi, and C. rosea as outgroup. Bootstrap support values are associated with lineages. Strain identifications are given after species names.

\section{Distribution of secondary metabolite gene clusters in Fusarium}

Generally, the biosynthesis genes for fungal secondary metabolites are located in clusters that can span more than 10,000 bases (Brakhage, 2013). In most fungal genomes, the clusters contain one or several central biosynthesis genes encoding extremely large multidomain, multimodular enzymes belonging to the polyketide synthases (PKSs) or non-ribosomal peptide synthetases (NRPSs). To link genes to secondary metabolites, we examined the genomes of six Fusarium species in silico. The analysis revealed multiple genes clusters across the six species, with the highest number encoding for non-ribosomal protein synthetases (nrps), type 1-polyketide synthases (t1-pks), terpenes and other secondary metabolites (Table S3). The wholegenome analysis of $F$. culmorum predicted a total of 41 gene clusters, comprising 9 nrps, 2 hybrid nrps-type 1 pks, 6 type 1 pks, 1 hybrid type 1 pks-terpene, 1 type 3 pks, 9 terpenes, 1 terpene-type 1 pks, 2 type 1 pks-nrps, 3 fatty acids and 7 gene clusters classified as other (Table S3). In this study, we focused mainly on predicted clusters that may play a role in pathogenicity and communication with other microorganisms, such as the genes encoding for mycotoxins and terpenes. 


\section{Genes encoding for mycotoxins}

The major mycotoxins produced by F. culmorum strains are the trichothecenes deoxynivalenol (DON), nivalenol (NIV) and the polyketide zearalenone (ZEN) (Wagacha \& Muthomi, 2007). Many of the trichothecene biosynthesis genes are located in a gene cluster comprising at least 10 genes: trichodiene synthetase (Tri5), P450 oxygenases (Tri4 and Tri11), acetyltransferase (Tri3), a transcription factor (Tri6), a toxin efflux pump (Tri12), and several unidentified hypothetical proteins (Tri7, Tri8, Tri9, and Tri10). We identified all Tri genes in the genome of F. culmorum with identity levels from $77-98 \%$ at the amino acid level, except for Tri7 and Tri9, and Tri13, which was only partially identified (Fig. 5, Table S4). The order and transcription directions of the open reading frames (ORFs) were identical for the gene cluster described in F. graminearum. It has been shown that the Tri13 and Tri7 genes are required for the production of nivalenol and 4-acetyl nivalenol, dividing the producing Fusarium isolates into DON and NIV chemotypes. Thus the partial presence of Tri13 and absence of Tri7 suggests that the PV F. culmorum isolate belongs to the DON chemotype, able to produce DON but unable to convert this to NIV. A recent survey investigating the spatial and temporal distribution of Fusarium chemotypes across Europe showed that in F. culmorum the prevalent chemotype was DON (Pasquali et al., 2016).

Another important Fusarium mycotoxin is ZEN. The ZEN gene cluster was described in F. graminearum, showing that four genes are essential for ZEN biosynthesis: the two polyketide synthases, PKS13 and PKS4, the alcohol oxidase ZEB1, and the transcription factor ZEB2 (Kim et al., 2005). We identified all four genes in the genome of $F$. culmorum, including several other genes located upstream and downstream with identity levels from 82-96\% at the amino acid level (Fig. 4, Table S4), suggesting that F. culmorum strain PV is a ZEN producer.

To confirm the production of DON and ZEN we determined the mycotoxin produced by F. culmorum strain PV upon infection of maize and wheat grains. 


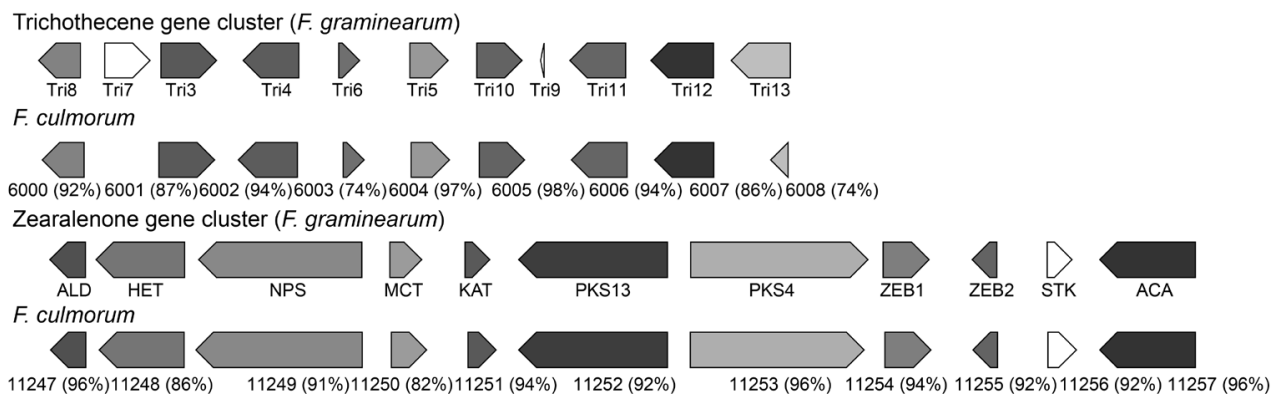

Fig. 4 / Mycotoxin gene clusters in F. culmorum. Locus tags are indicated for each gene along with the percentage of amino acid sequence identity to triochothecene and zearalenone gene clusters in $F$. graminearum.

\section{Mycotoxin production during plant infection}

Mycotoxins play important roles in the pathogenesis of Fusarium. Thus, we measured the mycotoxin levels in maize and wheat under infection with F. culmorum. Infection of maize and wheat grains resulted in high levels of several mycotoxins (Fig. 5, Table S4). High levels of DON (up to $251 \mathrm{mg} / \mathrm{kg}$ in maize and up to $256 \mathrm{mg} / \mathrm{kg}$ in wheat) and ZEN (up to $150 \mathrm{mg} / \mathrm{kg}$ in maize and up to $230 \mathrm{mg} / \mathrm{kg}$ in wheat) were detected (Fig. 5, Table S4). DON concentration was highest in F. culmorum infected maize, while ZEN concentration was highest in F. culmorum infected wheat. Traces of HT2toxin, T2-toxin, Fumonisin B1, Fumonisin B2 and Ochratoxin A were present, but were not detected in any significant amounts as compared to the control. These results confirm that $F$. culmorum strain PV produces DON and ZEN at high levels.
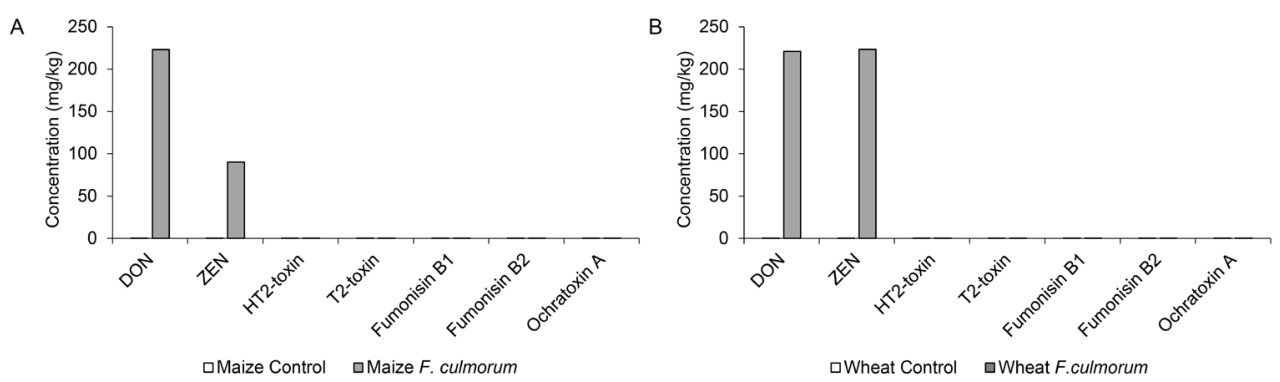

Fig. 5 / Mean mycotoxin concentration ( $\mathrm{mg} / \mathrm{kg}$ ) after three weeks of infection with F. culmorum in wheat (A) and maize (B). DON: Deoxynivalenol, ZEN: Zearalenone 


\section{Genes encoding for terpene synthases and terpene products}

Terpene synthases are a highly specialised group of enzymes that catalyse the formation of a wide range of terpenes (Dickschat, 2016). In order to link genes encoding for terpenes, we first looked at amino acid sequences of terpene synthases in the genome of F. culmorum. By blast comparison with known terpene synthases form other fungi we identified two enzymes, trichodiene and longiborneol synthase (Fig. 6). Using GC/MS identified a range of terpenes (Fig. 7A, B and C, Table 2), some of which have been identified in previous studies and linked to changes in motility in the interacting bacteria (Schmidt et al., 2015).

A peculiar feature of terpene synthases is that they typically produce multiple terpenes (Quin et al., 2014, Dickschat, 2016). It remains to be addressed in future studies, whether these two terpene synthases are the manufacturers of all identified terpenes or whether there are yet unidentified terpene synthases in the genome of $F$. culmorum. 


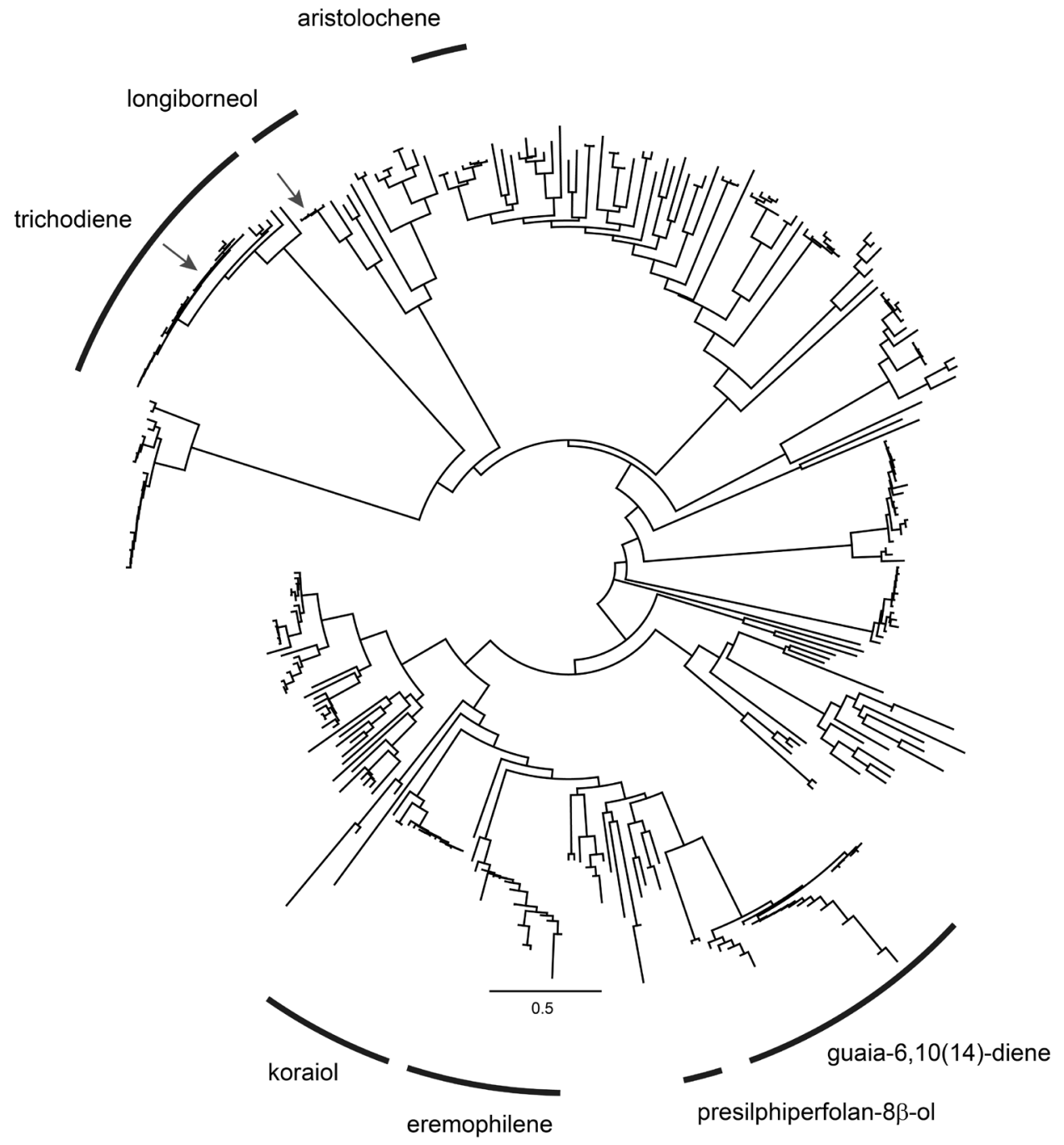

Fig. $\mathbf{6}$ / Phylogenetic tree of fungal terpene cyclases. The black bars indicate groups of highly homologous enzymes from which at least one enzyme was functionally characterised. The arrows point to the two enzymes from F. culmorum for trichodiene and longiborneol. 
A
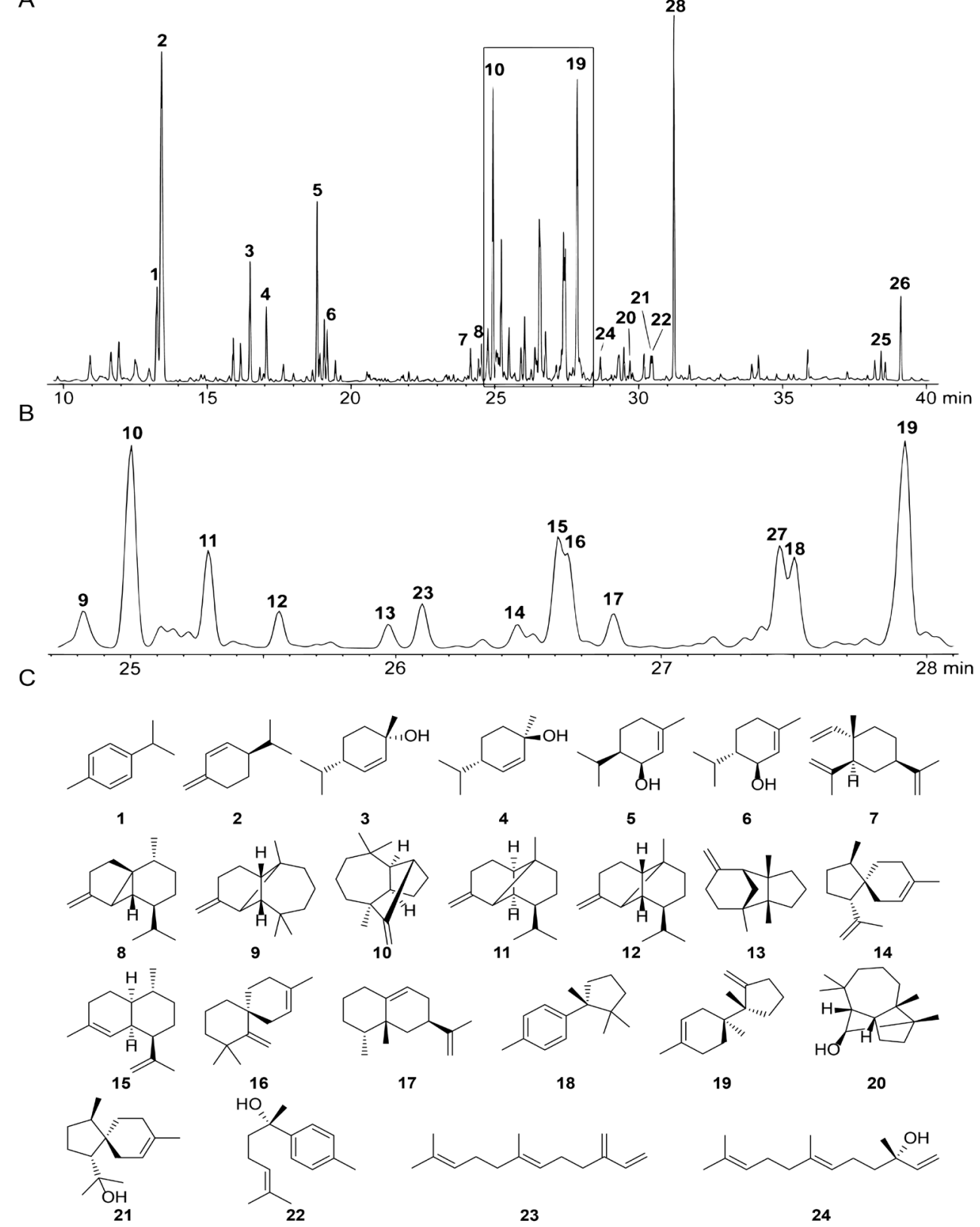

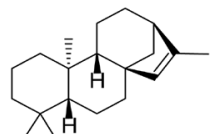

25

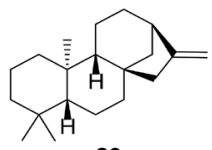

26

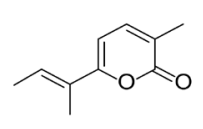

27

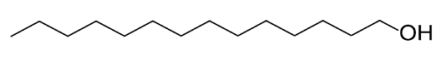

28 
Fig. 7 / (A) Chromatogram of all terpenes produced by F. culmorum according to numbers in Table 2 (B) Zoomed in chromatogram of major terpenes in F. culmorum (C) Structures of identified terpenes

Table $\mathbf{2}$ I Identified terpenes in F. culmorum. I: Experimental retention indices, I (Lit.): Retention indices found in literature.

\begin{tabular}{|c|c|c|c|}
\hline F. culmorum PV & RT [min] & $\mathbf{I}$ & I (Lit.) \\
\hline Benzaldehyde & 10.95 & 959 & 952 \\
\hline 1-Octen-3-ol & 11.67 & 979 & 974 \\
\hline 3-Octanone & 11.92 & 982 & 979 \\
\hline p-Cymene (1) & 13.27 & 1024 & 1026 \\
\hline$\beta$-Phellandrene (2) & 13.46 & 1029 & 1026 \\
\hline n-Nonanal & 15.96 & 1102 & 1100 \\
\hline Phenylethyl alcohol & 16.21 & 1110 & 1106 \\
\hline cis-p-Menth-2-en-1-ol (3) & 16.54 & 1120 & 1118 \\
\hline trans-p-Menth-2-en-1-ol (4) & 17.10 & 1138 & 1136 \\
\hline cis-Piperitol (5) & 18.87 & 1194 & 1195 \\
\hline trans-Piperitol (6) & 19.22 & 1205 & 1207 \\
\hline$\beta$-Elemene (7) & 24.22 & 1383 & 1389 \\
\hline$\beta$-Cubebene (8) & 24.49 & 1393 & 1390 \\
\hline$\beta$-Longipinene (9) & 24.82 & 1405 & 1405 \\
\hline Longifolene (10) & 25.00 & 1412 & 1413 \\
\hline$\beta$-Ylangene (11) & 25.29 & 1423 & 1425 \\
\hline$\beta$-Copaene (12) & 25.55 & 1434 & 1430 \\
\hline ß-Barbatene (13) & 25.98 & 1450 & 1445 \\
\hline (E)- $\beta$-Farnesene (23) & 26.10 & 1455 & 1454 \\
\hline$\beta$-Acoradiene (14) & 26.45 & 1469 & 1469 \\
\hline Amorpha-4,11-diene (15) & 26.61 & 1475 & 1472 \\
\hline Isochamigrene (16) & 26.64 & 1476 & \\
\hline 5-epi-Aristolochene (17) & 26.82 & 1483 & 1477 \\
\hline Gibepyrone A (27) & 27.45 & 1508 & 1509 \\
\hline Cuparene (18) & 27.50 & 1510 & 1504 \\
\hline Trichodiene (19) & 27.93 & 1528 & 1533 \\
\hline (E)-Nerolidol (24) & 28.72 & 1561 & 1561 \\
\hline Hexadecane & 29.56 & 1596 & 1600 \\
\hline Longiborneol (20) & 29.76 & 1605 & 1607 \\
\hline B-Acorenol (21) & 30.50 & 1637 & 1636 \\
\hline Gossonorol (22) & 30.55 & 1639 & 1636 \\
\hline
\end{tabular}




\begin{tabular}{llll} 
Tetradecanol (28) & 31.28 & 1671 & 1671 \\
Heptadecane & 31.83 & 1695 & 1700 \\
Octadecane & 34.00 & 1795 & 1800 \\
Kaur-15-ene (24) & 38.29 & 2007 & 1997 \\
Kaur-16-ene (26) & 39.19 & 2056 & 2049 \\
\hline
\end{tabular}

\section{Identification of secondary metabolites using LESA and UPLC-MS/MS}

To get an overview of water-soluble secondary metabolites produced F. culmorum, we employed two complementary approaches LESA and UPLC-MS/MS. In total, 15 metabolites were identified (Table 3). Among those were several known pigments like aurofusarin, rubrofusarin and nor-rubrofusarin. Aurofusarin, a red polyketide pigment, was first isolated from F. culmorum by Ashley et al. (1937) and was shown to have antibiotic properties against both mycelial fungi and yeasts. Rubrofusarin, an orange polyketide pigment, is reported to be produced by various fungi, including F. graminearum, Aspergillus niger, A. parasiticus and Ustilaginoidea virens, and was shown to inhibit the human DNA topoisomerase II-a and to have general antibiotic effects on Mycobacterium tuberculosis and various filamentous fungi (Rugbjerg et al., 2013). The orange polyketide pigment nor-rubrofusarin is an intermediate of the rubrofusarin biosynthesis pathway, which is methylated to yield rubrofusarin (Rugbjerg et al., 2013). These pigments give the characteristic appearance of $F$. culmorum on the macroscopic scale and possibly contribute to its protection to secondary metabolites produced by other microorganisms in its surrounding.

Further, several mycotoxins were identified. The mycotoxins fumonisin B2 is commonly produced by Fusarium sp. and infect maize, wheat and other cereals. Butenolide is a Fusarium mycotoxin frequently detected from cereal grains in endemic areas (Wang et al., 2009). Culmorin and Culmorone belong to the class of Culmorins, which are tricyclic sesquiterpene diols that have been reported from $F$. culmorum, F. graminearum, F. venenatum and have antifungal activity (McCormick et al., 2010). Fusarin A is a polyketide that belongs to the class of Fusarins (DiazSanchez et al., 2012)2012. T-2 toxin and its deacetylated form HT-2 toxin belong to the group of trichothecene mycotoxins that have been shown to not only inhibit protein synthesis and cell proliferation in plants, but also cause acute or chronic intoxication of humans and animals (Rocha et al., 2005). Furthermore, neosolaniol (NEO), diacetoxyscirpenol (DAS) and monoacetoxyscirpenol (MAS) are potent trichothecene mycotoxins. The trichothecenes Deoxynivalenol (DON), and 
its acetylated derivate 3-acetyl DON (3-ADON) as well as NIV and ZEN are potent mycotoxins that are the major mycotoxins of Fusarium.

We employed a new technique, LESA that allows direct detection of compounds at concentrations below the detection level of other methods, such as standard LC-MS/MS measurements used in this study for the detection of mycotoxins. Processing of the samples may lead to degradation of certain compounds, while LESA allows detection directly from an agar plate and was successfully used for monitoring metabolites produced by interacting fungi (Menezes et al., 2015). Combined with the high sensitivity of the LESA method, we could not only confirm the mycotoxins detected with LC-MS/MS, but also detected a range of additional mycotoxins. An important factor influencing the production of secondary metabolites is the medium employed (Hestbjerg et al., 2002)2002. Here, we used a nutrient-rich medium, while for LC-MS/MS analysis the metabolites were detected from grains infected with F. culmorum. The difference in the medium may contribute to the difference in detected metabolites. The detected mycotoxins are likely to contribute to the high pathogenicity of F. culmorum in several crops, including maize, barley and wheat. 
Table $\mathbf{3}$ / Secondary metabolites of F. culmorum detected by UPLC-MS

\begin{tabular}{|c|c|c|c|c|c|}
\hline$\#$ & Metabolite & $\begin{array}{l}\text { Molecular } \\
\text { Formula }\end{array}$ & $\begin{array}{l}{[\mathrm{M}+\mathrm{H}]+\text { or }} \\
*[\mathrm{M}-\mathrm{H}]-\end{array}$ & $\begin{array}{l}\text { Mass error } \\
(\triangle \mathrm{ppm})\end{array}$ & $\begin{array}{l}\text { Chemical } \\
\text { classification }\end{array}$ \\
\hline 1 & Aurofusarin & $\mathrm{C}_{30} \mathrm{H}_{18} \mathrm{O}_{12}$ & 571.08575 & -2.368 & Polyketide \\
\hline 2 & Rubrofusarin & $\mathrm{C}_{15} \mathrm{H}_{12} \mathrm{O}_{5}$ & 273.07586 & 0.659 & Polyketide \\
\hline 3 & Nor-rubrofusarin & $\mathrm{C}_{14} \mathrm{H}_{10} \mathrm{O}_{5}$ & 259.05997 & & Polyketide \\
\hline 4 & Fumonisin B2 & $\mathrm{C}_{34} \mathrm{H}_{59} \mathrm{NO}_{14}$ & *704.3855 & -1.119 & Polyketide \\
\hline 5 & Butenolide & $\mathrm{C}_{6} \mathrm{H}_{7} \mathrm{NO}_{3}$ & 142.04999 & 0.847 & Polyketide \\
\hline 6 & culmorin & $\mathrm{C}_{15} \mathrm{H}_{26} \mathrm{O}_{2}$ & 239.20061 & 0.223 & Polyketide \\
\hline 7 & culmorone & $\mathrm{C}_{15} \mathrm{H}_{24} \mathrm{O}_{2}$ & 237.18468 & -0.955 & Polyketide \\
\hline 8 & Fusarin A & $\mathrm{C}_{23} \mathrm{H}_{29} \mathrm{NO}_{6}$ & 416.20679 & 0.062 & Polyketide \\
\hline 9 & Fusamarin & $\mathrm{C}_{18} \mathrm{H}_{28} \mathrm{O}_{4}$ & 309.20627 & 0.757 & Polyketide \\
\hline 10 & $\mathrm{~T}-2$ & $\mathrm{C}_{24} \mathrm{H}_{34} \mathrm{O}_{9}$ & 467.2261 & -3.123 & Terpene \\
\hline 11 & HT-2 & $\mathrm{C}_{22} \mathrm{H}_{32} \mathrm{O}_{8}$ & 425.21667 & -0.763 & Terpene \\
\hline 12 & neosolaniol (NEO) & $\mathrm{C}_{19} \mathrm{H}_{26} \mathrm{O}_{8}$ & 383.17004 & 0.062 & Terpene \\
\hline 13 & diacetoxyscirpenol (DAS) & $\mathrm{C}_{19} \mathrm{H}_{26} \mathrm{O}_{7}$ & 367.17523 & 0.273 & Terpene \\
\hline 14 & monoacetoxyscirpenol (MAS) & $\mathrm{C}_{17} \mathrm{H}_{24} \mathrm{O}_{6}$ & 325.16485 & 0.877 & Terpene \\
\hline 12 & deoxynivalenol (DON) & $\mathrm{C}_{15} \mathrm{H}_{20} \mathrm{O}_{6}$ & 297.13326 & -0.016 & Terpene \\
\hline 13 & 3-acetyl DON & $\mathrm{C}_{17} \mathrm{H}_{22} \mathrm{O}_{7}$ & 339.14338 & -1.325 & Terpene \\
\hline 14 & nivalenol (NIV) & $\mathrm{C}_{15} \mathrm{H}_{20} \mathrm{O}_{7}$ & *311.11393 & 0.977 & Terpene \\
\hline 15 & zearalenone (ZEN) & $\mathrm{C}_{18} \mathrm{H}_{22} \mathrm{O}_{5}$ & 319.15411 & 0.344 & Polyketide \\
\hline
\end{tabular}

\section{Concluding remarks}

Here we describe for the first time the genome of $F$. culmorum and its secondary metabolome. This F. culmorum isolate was shown to be highly pathogenic to maize, wheat and barley and to produce a wide range of secondary metabolites, including volatile terpenes and mycotoxins.

Sequencing of the F. culmorum genome and establishment of its predicted secondary metabolite clusters provides a starting point for future investigations regarding the ecology of this important species. We show the close relatedness between F. culmorum and F. graminearum, which is also evident in the presence and structure of the DON and ZEN biosynthetic gene clusters in the two species. 


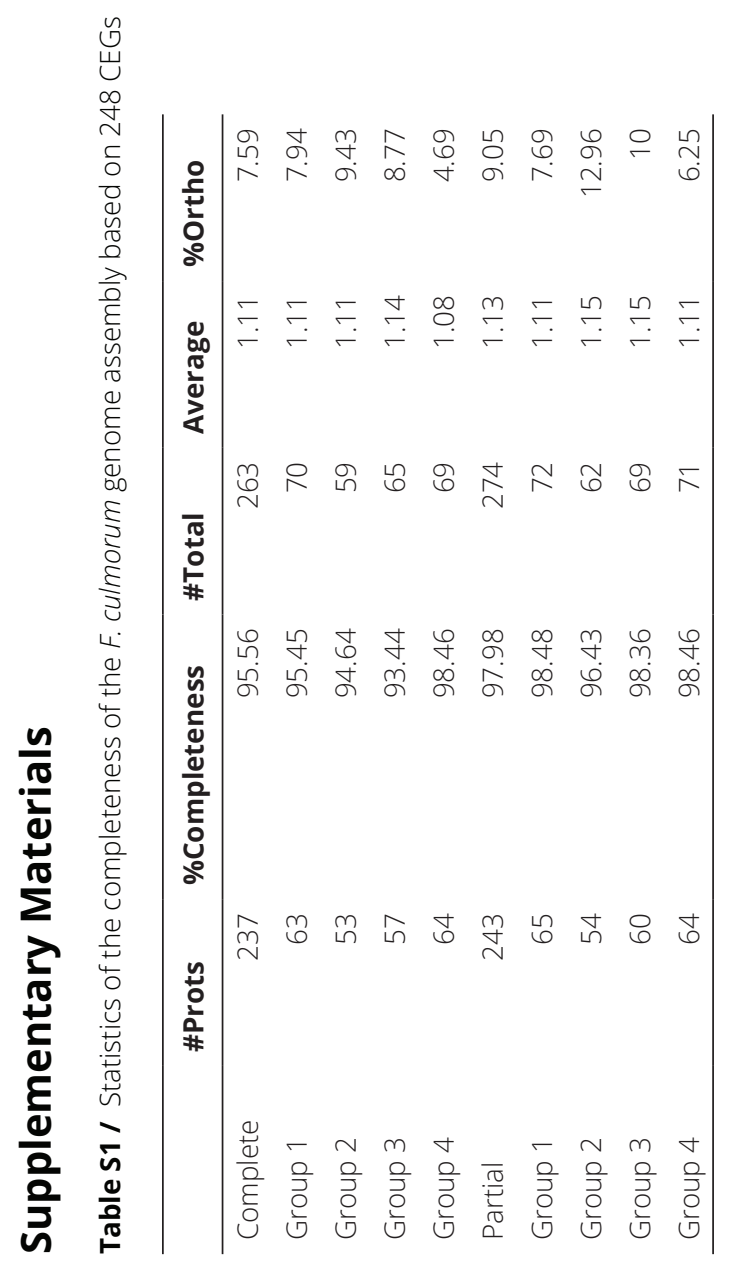

167 / Chapter 6 


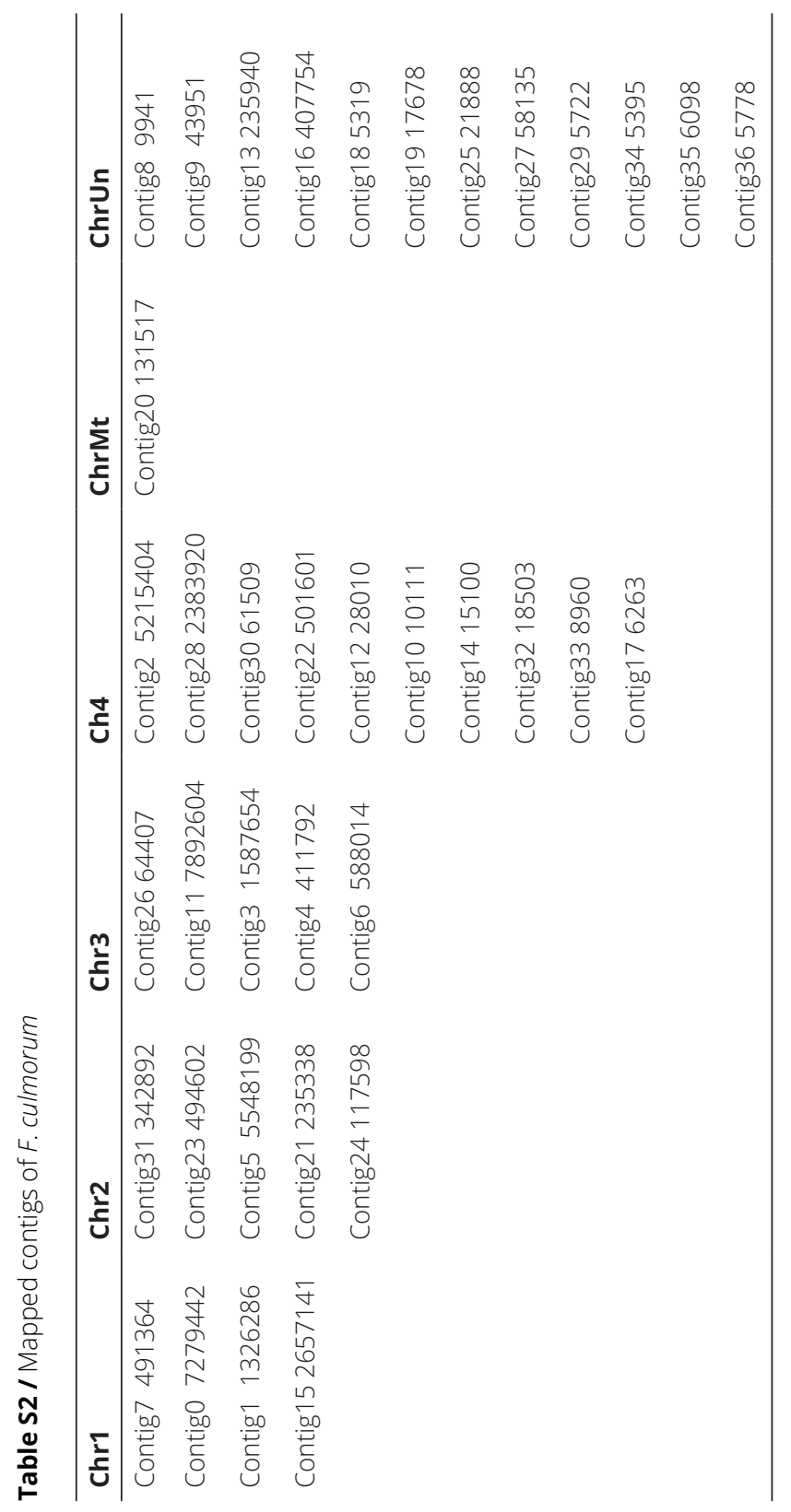




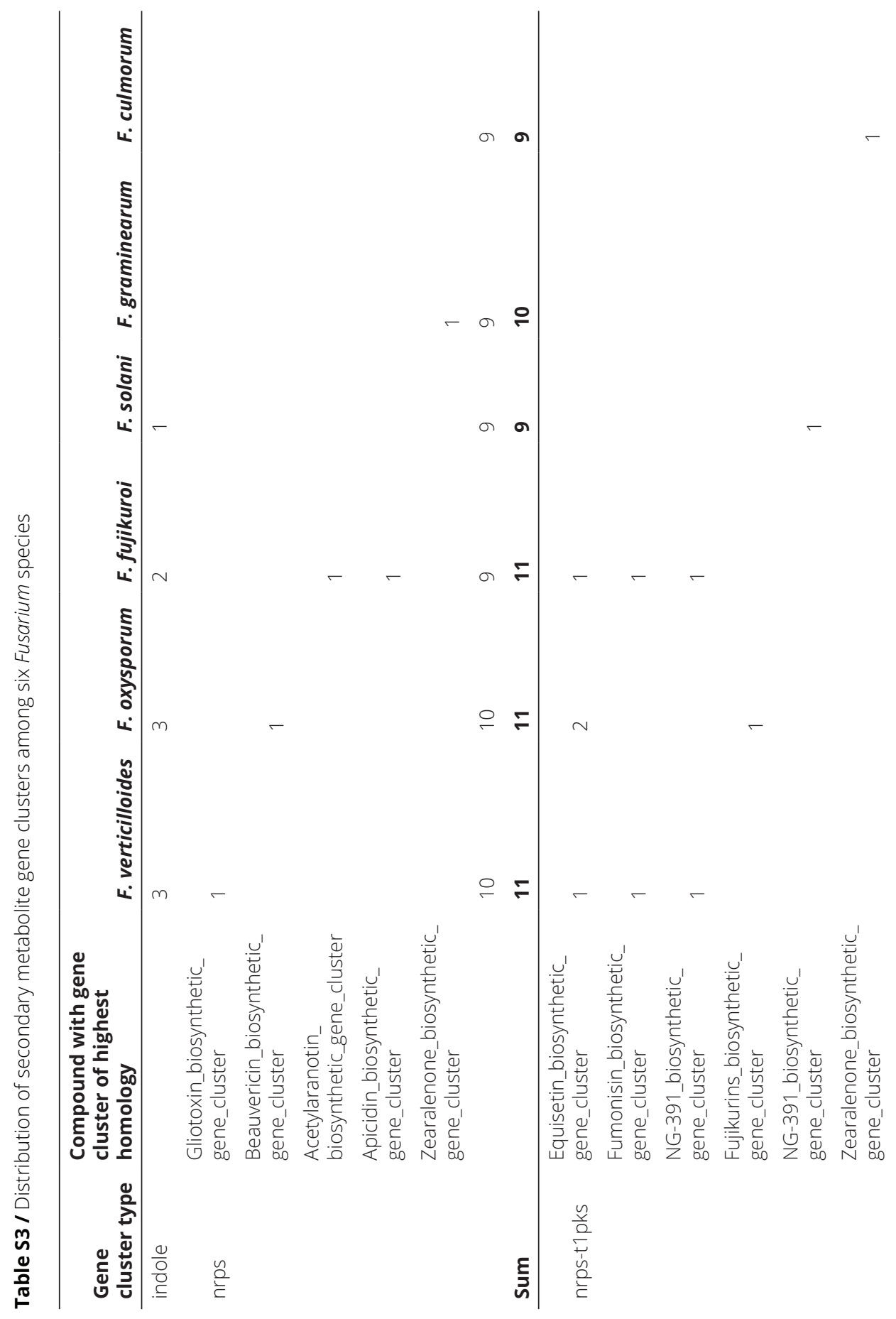




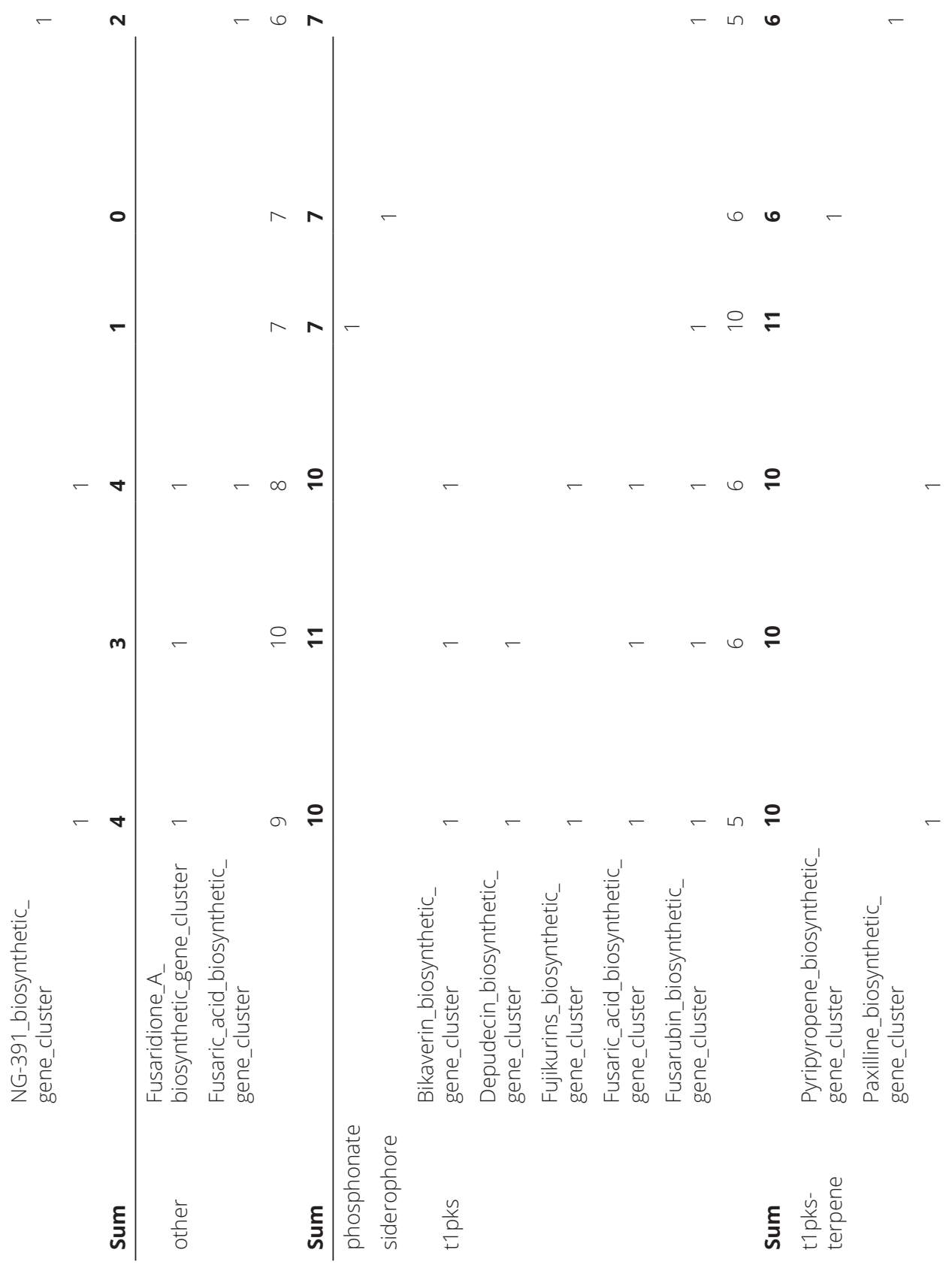



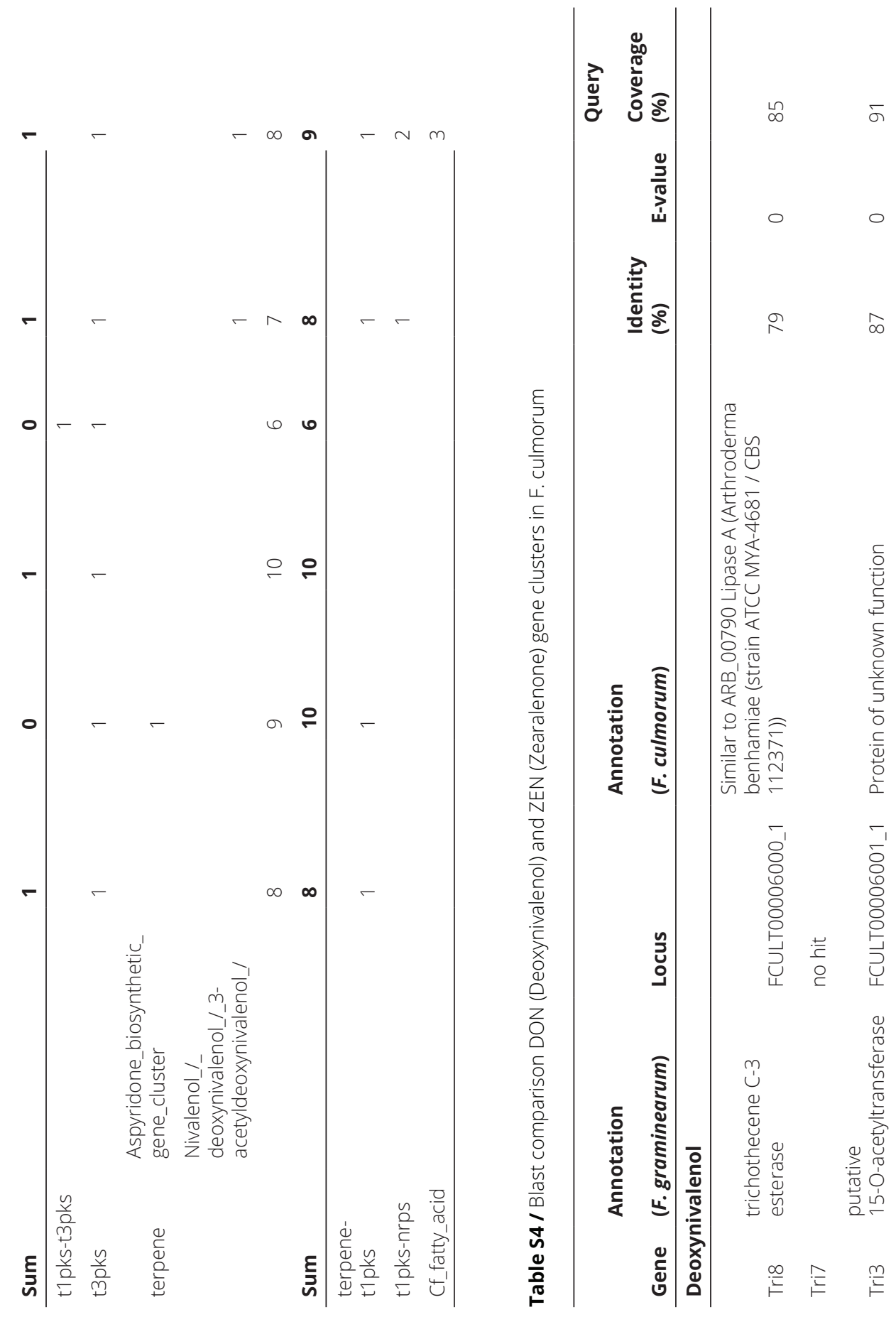


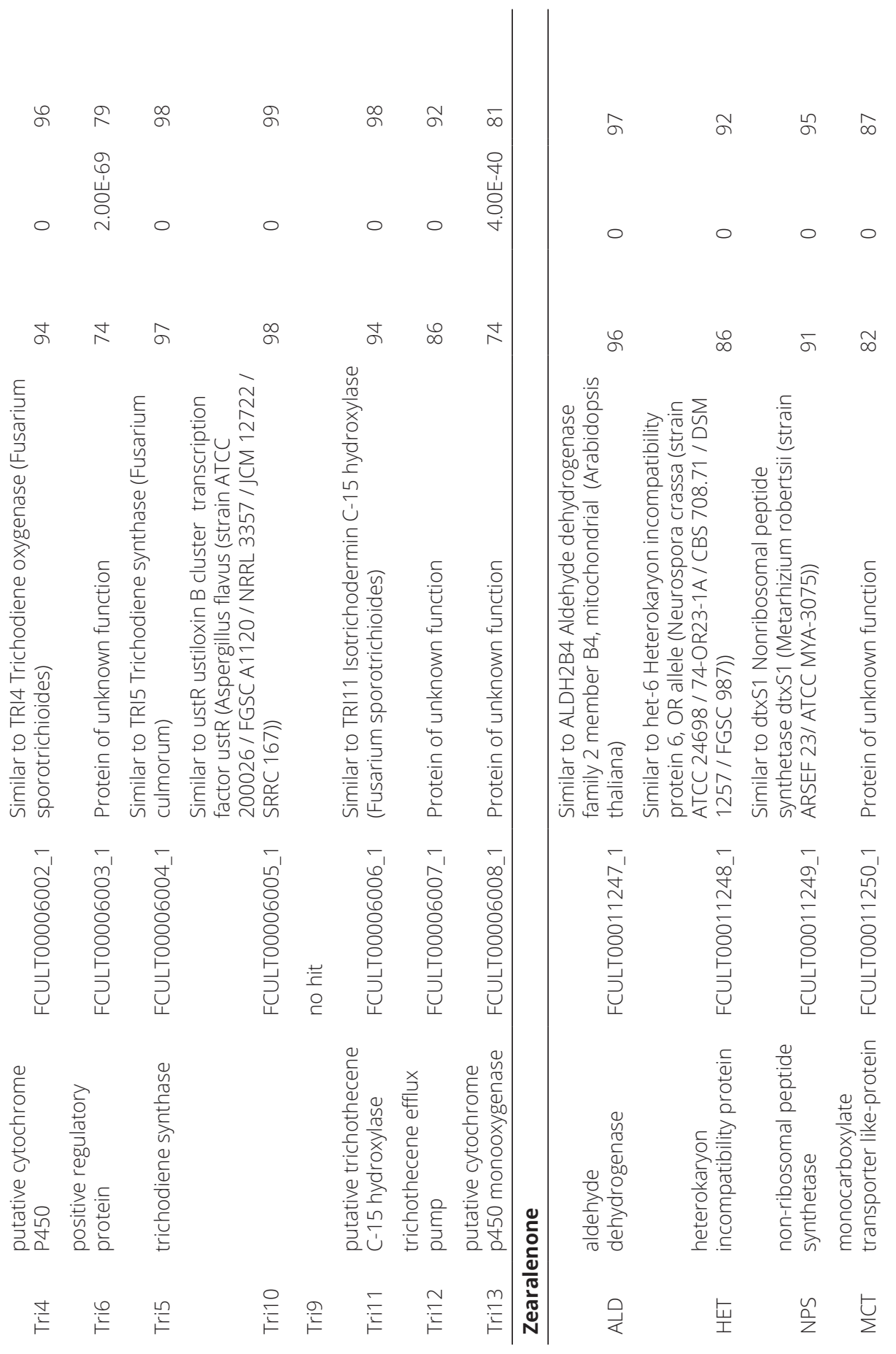




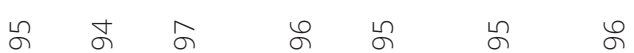

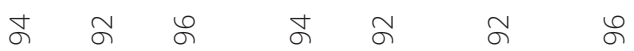

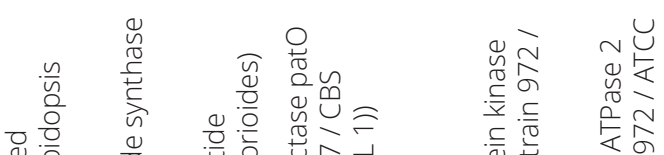

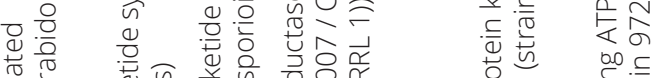

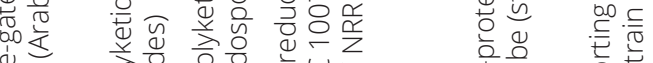

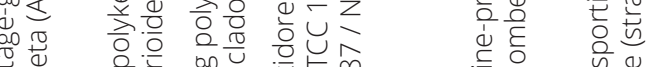

I

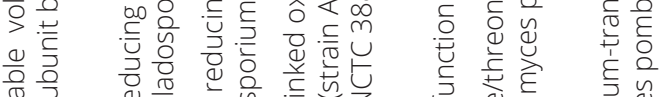

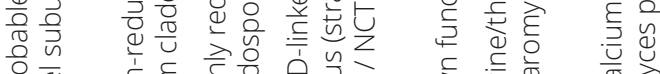

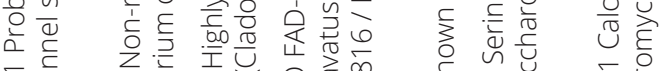

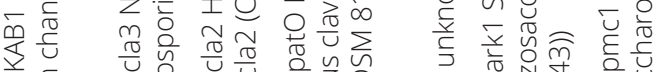

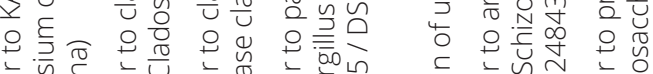

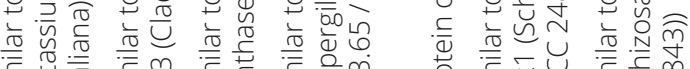

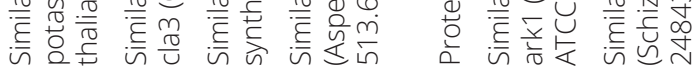

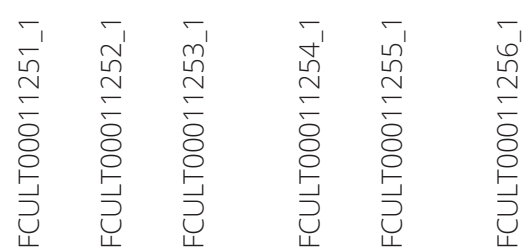

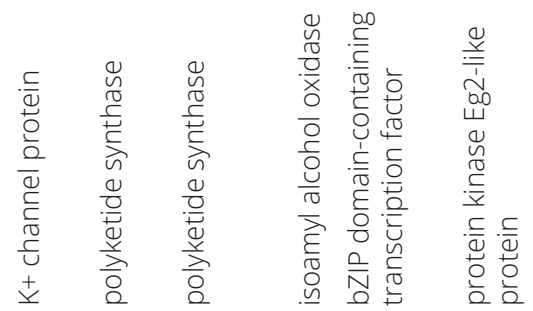

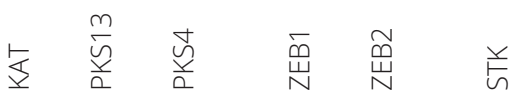

ค |

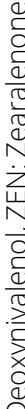

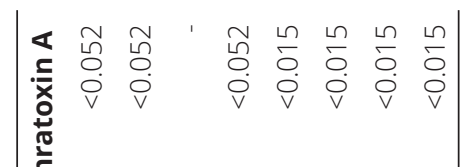

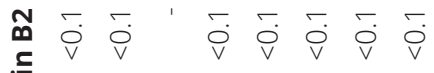

$\frac{5}{2}$

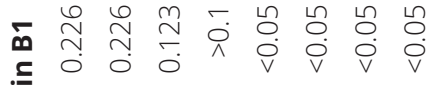

$\frac{. \bar{n}}{\frac{2}{2}}$

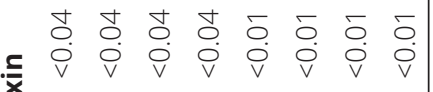

ำ

논

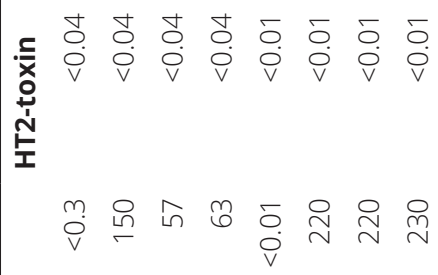

岕

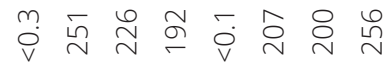

zํㅡㅁ

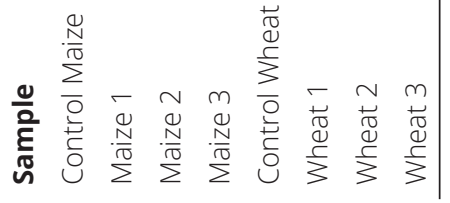


174 / Chapter 6 


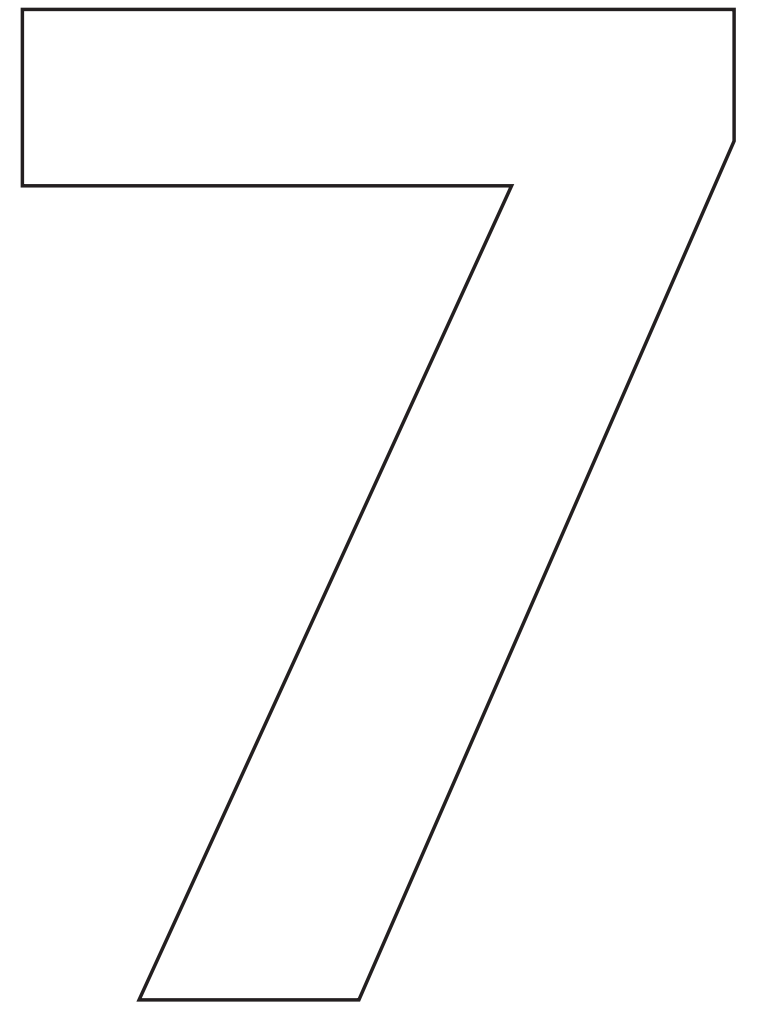

General discussion 



\section{Volatile affairs in microbial interactions}

Interactions and communication among organisms are fundamental for the functioning of any ecosystem. The essential role of volatile organic compounds (VOCs), also known as infochemicals, in the communication between organisms has been acknowledged for more than 30 years (Dicke \& Sabelis, 1988). However, their ecological functions have been mainly studied for aboveground plant-plant and plant-insect interactions (Baldwin et al., 2006, Babikova et al., 2013). In recent years, there has been a reviving interest in microbial VOCs in the field of microbial ecology. Due to their unique nature they have been shown to play central roles in the long-distance interaction and communication within the microbial world (Schmidt et al., 2015). The overall aims of this thesis were to i) characterize VOCs emitted by plant pathogenic and saprotrophic fungi ii) investigate their effects on bacteria iii) understand molecular mechanisms underlying the bacterial perception and response to fungal VOCs, and iv) identify genes and biosynthetic pathways underlying VOC production in both bacteria and fungi. Here I discuss the major findings of this thesis in the context of related published literature. Furthermore, I will raise new propositions on the ecological role of VOCs in soil environments from the microbe's point of view.

\section{Microbe's talk: Volatile-mediated communication between fungi and bacteria}

Bacteria and fungi produce a range of structurally and functionally diverse VOCs (Effmert et al., 2012). Several studies have addressed the role of VOCs from a bacterial point of view, acting as infochemical molecules in soil or protecting plants against pathogenic fungi and oomycetes (Garbeva et al., 2014, Cordovez et al., 2015, De Vrieze et al., 2015). Yet, very little is known about the chemical diversity of VOCs produced by filamentous eukaryotic microbes (fungi and oomycetes) as well as their ecological role in fungal-bacterial interactions.

VOC profiling of five soil/rhizospheric fungi and an oomycete revealed that each microbe has its own chemical signature and that both the growth stage and nutritional status (rich vs. poor media) have a strong effect on VOC emission (Chapter $\mathbf{3}$ ). These results suggest that VOC production by fungi and oomycetes is tightly controlled in time according to soil nutritional constraints (Hacquard, 2017). Since organic carbon represents the most important nutrient source in soil, the 
production of VOCs is strongly influenced by the availability of organic substrates. This appears to be the case for particular terpenes, whose emission was enhanced under nutrient-poor conditions. These findings make it tempting to speculate that filamentous microbes adjust the production of VOCs for communication with other microbes depending on the nutrient status. Taking it one step further, it is an obvious question what ecological effects those VOCs have on the behavior of the interacting organism. By testing their effect on bacterial traits I found that VOCs emitted by particular fungi/oomycetes strongly affect the motility of two bacterial isolates (Collimonas pratensis and Serratia plymuthica). This suggests that, similar to bacterial VOCs that have been shown to alter specific fungal/oomycetal traits (Tyc et al., 2014, De Vrieze et al., 2015, Sharifi \& Ryu, 2016), VOCs produced by fungi/ oomycetes can be in turn sensed by bacteria, therefore modulating their ability to move. These results were confirmed when studying the bacterial transcriptome and proteome of S. plymuthica PRI-2C in response to fungal VOCs. Here, I showed that the bacterium responds to fungal VOCs with changes in gene and protein expression related to motility (Chapter 4). By testing pure compounds I could link the ability of bacteria to move to the production of fungal terpenes (Chapter $\mathbf{3}$ ). These results shed new lights into one possible mechanism used by particular soil and rhizospheric fungi/oomycetes to attract mutualistic or to fend off competitive bacterial neighbors by emitting specific terpenes as communication signals under specific nutrient conditions.

\section{Terpenes as a lingua franca between fungi and bacteria}

It is well established that plants emit VOCs as aboveground communication signals to "cry for help" when under attack (Dicke \& Sabelis, 1988). Also belowground, plant roots produce VOCs, including terpenes that are actively involved in rhizosphere communication (Van Tol et al., 2001, Rasmann et al., 2005). For example, when roots are damaged by insects, the sesquiterpene (E)- $\beta$-caryophyllene is emitted to attract entomopathogenic nematodes (Rasmann et al., 2005). Similarly, citrus roots infested by root-feeding herbivores recruit entomopathogenic nematodes via the emission of several mono- and sesquiterpenes (Ali et al., 2010). In both cases, the nematode attractants appear to be volatile terpenes implying that terpenes are important signals in odor-mediated interactions aboveground as well as belowground. It is thus not surprising that, similar as for plants, terpenes may act as a lingua franca between bacteria and fungi. As described in Chapter 3, I showed that the plant 
pathogenic fungus F. culmorum emits a range of terpenes. Interestingly, I identified the unusual volatile terpene sodorifen that was produced by the bacterium in response to fungal VOCs (Chapter 4). Here I show for the first time that an actual dialogue is taking place between the emitting organism (fungus) and the receiving organism (bacterium), by sending a response back in form of a volatile terpene. One of the main questions that came up during the study was what the ecological function of this particular terpene is, which in previous studies on sodorifen has not been addressed (Domik et al., 2016). I tested the synthesized pure compound in several concentrations on the growth of F. culmorum as well as on the motility of S. plymuthica as potential inter- or intraspecific signaling compound. Unfortunately, so far, our results did not explain any of these effects. Another possible explanation may be that the compound does not act on the emitting organism, but on other member in the rhizosphere community, including bacteria, fungi or protists.

An additional aspect, that has been largely overlooked, is the effect of microbial terpenes on plants. As discussed earlier, plants send signals into the belowground world to attract beneficial microbes when under attack, however, whether microbes send signals to the plant is largely unknown. A limited number of studies showed that plants respond to VOCs produced by microorganisms by using $A$. thaliana as a model plant. These studies have shown that bacteria are able to modify the plant's root system and biomass production, ranging from increased biomass to plant death (Ryu et al., 2003, Vespermann et al., 2007, Blom et al., 2011), thus demonstrating that the physiological status of a plant is influenced by the presence of microbes belowground. However, these systems are difficult to transfer to real situations that prevail in nature. More recently, Sherif et al. (2016) showed that the volatile profiles of maize ears were influenced by the presence of different fungal pathogens, leading to the emission of common volatile markers, mostly composed of sesquiterpenoids. Based on these findings we could speculate that the plant and the rhizomicrobiome strongly influence each other via the secretion and detection of terpenes and thus communicate not only one-way between each other, but also sense and respond to each other within the plant-root network. However, these questions remain to be addressed in future studies.

\section{On the search for the bacterial "nose"}

Now that we have come to the understanding that bacteria and fungi communicate via VOCs, including terpenes, we still do not know exactly how VOCs are recognized 
and perceived by bacteria. The big challenge is to determine whether VOCs are internalized and transduced by receptor-mediated processes, whether they interact with the cell membrane to initiate signal transduction cascades or whether they are simply taken up by the cell and metabolized. The perception of VOCs could occur via changes in the electrochemical membrane potential or by receptors. For plants, the current view is that due to their lipophilic nature, VOCs such as monoand sesquiterpenes may interfere with membrane structures, thereby causing depolarization of the membranes and triggering $\mathrm{Ca}^{2+}$ signaling in plants (Maffei et al., 2001, Heil, 2014). Depolarization of the bacterial membrane have been shown for antimicrobial agents on both Gram-positive and Gram-negative bacteria (Epand et al., 2010), however VOCs, so far, have not been shown to promote such an effect. The same holds true for volatile receptors. There are numerous studies on olfactory receptors in insects and mammals aimed to understand the underlying principles and mechanisms of the sense of smell (Fleischer et al., 2009, Sato \& Touhara, 2009). In plants, ethylene receptors have been suggested as putative receptors in VOC perception (Ryu et al., 2003, Bailly \& Weisskopf, 2012). Yet, molecular receptors for the perception of terpenes, for example, have not yet been identified in plants or in bacteria. It is thus not surprising that the search for volatile receptors is one of the "hot topics" in the field.

In bacteria the interaction between terpenes and the bacterial cell has been mainly described as increased permeability of the cell membrane (Trombetta et al., 2005, Nazzaro et al., 2013). Kovac et al. (2015) described a concentration dependent effect of (-)-a-pinene on antimicrobial resistance in C. jejuni, leading to either inhibition of antimicrobial efflux in lower concentrations, while in higher concentrations leading to increased permeability of the cell membrane thereby promoting the influx of antimicrobials. I identified several up-regulated genes and proteins related to cell wall biogenesis in S. plymthica PRI-2C when exposed to fungal VOCS (Chapter 4). These results indicate that terpenes emitted by F. culmorum make the bacterial cell wall more permeable for VOCs to enter and to be taken up by the cell. As a result the bacterium activates membrane biosynthetic pathways when exposed to VOCs that cause damages in the bacterial cell wall and thus require repair. As receptors, two-component systems are important for bacteria to sense and respond to environmental signals. For example, Cheng et al. (2016) showed that the membrane bound sensor kinase GacS is involved in the regulation of the VOC production by the plant growth-promoting P. fluorescence SBW25. In 
Chapter 4, I found that the genome of S. plymuthica encodes for numerous twocomponent systems as well as for chemotaxis. I identified the sensor histidine kinase that upregulates transcription of small RNAs, and GIrK and the sensor protein QseC that activates the flagella regulon. Moreover, the methyl-accepting chemotaxis protein IV was identified, which acts as chemotactic-signal sensor that detects attractants and promotes bacterial movement towards suitable sites for colonization. These findings are one step further towards identifying mechanisms underlying the perception and response to VOCs. However, I was not able to find specific receptors for the perception of VOCs. Most likely, VOC perception occurs fast within a few hours, as recently shown for plants (Maffei et al., 2007). In our study I tested the response at two time points, without, including early time point responses. Hence, identification of specific receptors that play a role in the perception of VOCs remains an important issue for future research. These studies should go together with deciphering the biological relevant concentrations at which single or a combination of VOCs become reliable signals for the receiving organism.

\section{From gene to product: Bacteria and fungi as "treasure boxes" of terpenes}

Terpenes are generally considered to be plant metabolites. Recent research demonstrated that microorganisms, including fungi and bacteria, are a rich source of terpenes too (Rabe et al., 2013, Rabe \& Dickschat, 2013). In the past two decades several terpene cyclases and their related products have been characterised for several soil-derived fungi (Collado et al., 2007, Ebel, 2010, Singh et al., 2011, Dickschat, 2017). Yamada et al. (2015) showed that terpenes and their cyclases are also widespread in bacteria. Thus we can conclude that many soil microbes harbor genes encoding such terpene synthases. However, terpene synthases have been chemically characterized for only few bacteria and fungi. Although the principal processes of terpene biochemistry are well understood, it is challenging to predict terpene structures from the amino acid sequence of terpene synthases.

With the increasing availability of microbial genome sequences, we can now search through the genome data and look for gene sequences that likely code for terpene synthases. By using a comparative genomics approach, we found in Chapter $\mathbf{5}$ that Collimonas harbors two terpene synthase genes (CPter91_2617 and CPter291_2730). One of the crucial steps is to link genes to their products. As the product specificity of mono- and sesquiterpene cyclases cannot be predicted from their primary biochemical characterization, the two genes were expressed in 
E. coli and tested for cyclization reactions using FPP, GPP or GGPP as substrates. We found that both Collimonas enzymes converted FPP to a mix of sesquiterpenes and sesquiterpene alcohols, with the major peak being identified as germacrene D-4ol by, and several minor sesquiterpene peaks, which included $\delta$-cadinene. When GPP was applied as a substrate, the production of two monoterpenes identified as $\beta$-pinene and $\beta$-linalool was observed. The sesquiterpene products suggest that they are functionally related to plant and fungal cadinene/cadinol and germacrene D-4-ol synthases (Yoshikuni et al., 2006, Lauchli et al., 2014). Based on these findings, we suggest that there may be many new terpene products as yet undiscovered hiding in the genomes of bacteria.

In Chapter 6, I went one step further and used the fungal genome of our previous studies, Fusarium culmorum, to search for genes encoding for terpenes and other secondary metabolites that play important roles in fungal pathogenicity and in the interaction with other microbes. The genus Fusarium has been largely studied as a model for plant diseases as it represents an important group of fungal plant pathogens and for its health hazard posed to humans and livestock by $F$. culmorum mycotoxins. By sequencing the genome of F. culmorum and "searching" through the genome data for gene sequences that likely code for terpene synthases and other secondary metabolites, we identified two terpene synthase genes, longiborneol synthase and trichodiene synthase, as well as several genes encoding for mycotoxins. To link genes to their products, we used a combination of techniques, including, GC/MS and LESA a relatively new technique, combined with UPLC-MS/MS that allows detection of secondary metabolites directly from the Petri dish.

\section{Outlook: Towards unraveling the ecological role of terpenes and providing solutions for crop welfare}

The obtained results in this thesis show that both, bacteria and fungi, harbor genes for terpenes in their genomes and use them to communicate with each other. Within this thesis, I zoomed into one specific dialogue, taking place among fungi and bacteria, however the network of volatile communication is much larger and more complex. Thus, the next logical step towards unraveling the ecological role of terpenes is to discover their importance in shaping the plant holobiont, i.e. the plant and its associated microbiome. This will include studying the role of terpenes in microbe-microbe and plant-microbe interactions, by using a model plant. For our 
system maize (Zea mays) proves to be a suitable model plant, F. culmorum being a potent pathogen and S. plymuthica a plant beneficial bacterium that is potentially involved in boosting the plant's growth and health.

The obtained results of this thesis set the basis for further understanding the natural roles of terpene-mediated interactions, both below- and aboveground. Ultimately, this knowledge can be translated into innovative strategies for developments towards ecofriendly and sustainable agricultural practices. Bacterial and fungal VOCs, including terpenes, may represent an alternative to harmful pesticides, fungicides, and bactericides and thus might be the future to protect plants from pathogens and provide a setting for better crop welfare. Research in the last 10 years has led to an increasingly clear understanding of the role of VOCs as biocontrol agents to control various phytopathogens and as biofertilizers for plant growth promotion (Kanchiswamy et al., 2015). Yet, the application of microbial VOCs in agriculture is still in its infancy. Thus, further studies are needed to harness the potential of VOCs and to bring the knowledge from laboratory to field conditions. 
184 / Chapter 7 
/ References 



\section{A}

Adesina MF, Lembke A, Costa R, Speksnijder A \& Smalla K (2007) Screening of bacterial isolates from various European soils for in vitro antagonistic activity towards Rhizoctonia solani and Fusarium oxysporum: Site-dependent composition and diversity revealed. Soil Biology \& Biochemistry 39: 2818-2828.

Agnoli K, Lowe CA, Farmer KL, Husnain SI \& Thomas MS (2006) The ornibactin biosynthesis and transport genes of Burkholderia cenocepacia are regulated by an extracytoplasmic function sigma factor which is a part of the Fur regulon. J Bacteriol 188: 3631-3644.

Agren J, Sundstrom A, Hafstrom T \& Segerman B (2012) Gegenees: Fragmented Alignment of Multiple Genomes for Determining Phylogenomic Distances and Genetic Signatures Unique for Specified Target Groups. Plos One 7.

Aguilar C, Friscina A, Devescovi G, Kojic M \& Venturi V (2003) Identification of quorum-sensing-regulated genes of Burkholderia cepacia. Journal of Bacteriology 185: 6456-6462.

Ali JG, Alborn HT \& Stelinski LL (2010) Subterranean herbivore-induced volatiles released by citrus roots upon feeding by Diaprepes abbreviatus recruit entomopathogenic nematodes. J Chem Ecol 36: 361-368.

Andrews SC (1998) Iron storage in bacteria. Adv Microb Physiol 40: 281-351.

Ashburner M, Ball CA, Blake JA, et al. (2000) Gene ontology: tool for the unification of biology. The Gene Ontology Consortium. Nature genetics 25: 25-29.

Ashley JN, Hobbs BC \& Raistrick H (1937) Studies in the biochemistry of micro-organisms: The crystalline colouring matters of Fusarium culmorum (W. G. Smith) Sacc. and related forms. The Biochemical journal 31: 385-397.

Babikova Z, Gilbert L, Bruce TJ, Birkett M, Caulfield JC, Woodcock C, Pickett JA \& Johnson D (2013) Underground signals carried through common mycelial networks warn neighbouring plants of aphid attack. Ecology letters 16: 835-843.

\section{B}

Baer P, Rabe P, Fischer K, Citron CA, Klapschinski TA, Groll M \& Dickschat JS (2014) Induced-fit mechanism in class I terpene cyclases. Angew Chem Int Ed Eng/ 53: 7652 7656. 
Bailly A \& Weisskopf L (2012) The modulating effect of bacterial volatiles on plant growth: current knowledge and future challenges. Plant Signal Behav 7: 79-85.

Baldwin IT, Halitschke R, Paschold A, von Dahl CC \& Preston CA (2006) Volatile signaling in plant-plant interactions: "talking trees" in the genomics era. Science 311: 812-815.

Blin K, Medema MH, Kazempour D, Fischbach MA, Breitling R, Takano E \& Weber T (2013) antiSMASH 2.0--a versatile platform for genome mining of secondary metabolite producers. Nucleic Acids Res 41: W204-212.

Blom D, Fabbri C, Connor EC, Schiestl FP, Klauser DR, Boller T, Eberl L \& Weisskopf $L$ (2011) Production of plant growth modulating volatiles is widespread among rhizosphere bacteria and strongly depends on culture conditions. Environ Microbiol 13: 3047-3058.

Boisvert S, Laviolette F \& Corbeil J (2010) Ray: simultaneous assembly of reads from a mix of high-throughput sequencing technologies. Journal of computational biology : a journal of computational molecular cell biology 17: 1519-1533.

Brakhage AA (2013) Regulation of fungal secondary metabolism. Nat Rev Micro 11: 21-32.

Braun V, Mahren S \& Ogierman M (2003) Regulation of the Fecl-type ECF sigma factor by transmembrane signalling. Curr Opin Microbio/ 6: 173-180.

Brock NL, Huss K, Tudzynski B \& Dickschat JS (2013) Genetic dissection of sesquiterpene biosynthesis by Fusarium fujikuroi. Chembiochem : a European journal of chemical biology 14: 311-315.

Burkhardt I, Siemon T, Henrot M, Studt L, Rosler S, Tudzynski B, Christmann M \& Dickschat JS (2016) Mechanistic Characterisation of Two Sesquiterpene Cyclases from the Plant Pathogenic Fungus Fusarium fujikuroi. 55: 8748-8751.

Cane DE \& Ikeda H (2012) Exploration and mining of the bacterial terpenome. Accounts of chemical research 45: 463-472.

Cane DE, Xue Q \& Fitzsimons BC (1996) Trichodiene synthase. Probing the role of the highly conserved aspartate-rich region by site-directed mutagenesis. Biochemistry 35: 12369-12376. 
Chang WT, Chen CS \& Wang SL (2003) An antifungal chitinase produced by Bacillus cereus with shrimp and crab shell powder as a carbon source. Curr Microbiol 47: 102-108.

Cheng X, Cordovez V, Etalo DW, van der Voort M \& Raaijmakers JM (2016) Role of the GacS Sensor Kinase in the Regulation of Volatile Production by Plant Growth-Promoting Pseudomonas fluorescens SBW25. Frontiers in plant science 7.

Chernin L, Ismailov Z, Haran S \& Chet I (1995) Chitinolytic Enterobacter agg/omerans antagonistic to fungal plant-pathogens. Appl Environ Microb 61: 1720-1726.

Chin CS, Alexander DH, Marks P, et al. (2013) Nonhybrid, finished microbial genome assemblies from long-read SMRT sequencing data. Nat Methods 10: 563-+.

Choi KH \& Schweizer HP (2005) An improved method for rapid generation of unmarked Pseudomonas aeruginosa deletion mutants. Bmc Microbiol 5: 30.

Choi KH, Kumar A \& Schweizer HP (2006) A 10-min method for preparation of highly electrocompetent Pseudomonas aeruginosa cells: Application for DNA fragment transfer between chromosomes and plasmid transformation. J Microbiol Meth 64: 391-397.

Chu BC, Garcia-Herrero A, Johanson TH, Krewulak KD, Lau CK, Peacock RS, Slavinskaya Z \& Vogel HJ (2010) Siderophore uptake in bacteria and the battle for iron with the host; a bird's eye view. Biometals 23: 601-611.

Cianciotto NP (2009) Many substrates and functions of type II secretion: lessons learned from Legionella pneumophila. Future Microbiol 4: 797-805.

Collado IG, Sanchez AJ \& Hanson JR (2007) Fungal terpene metabolites: biosynthetic relationships and the control of the phytopathogenic fungus Botrytis cinerea. Nat Prod Rep 24: 674-686.

Cordovez V, Carrion VJ, Etalo DW, Mumm R, Zhu H, van Wezel GP \& Raaijmakers JM (2015) Diversity and functions of volatile organic compounds produced by Streptomyces from a disease-suppressive soil. Frontiers in microbiology 6: 1081.

Cornelis GR \& Wolf-Watz H (1997) The Yersinia Yop virulon: a bacterial system for subverting eukaryotic cells. Mol Microbiol 23: 861-867. 


\section{D}

Dahiya N, Tewari R, Tiwari R \& Hoondal G (2005) Production of an antifungal chitinase from Enterobacter sp NRG4 and its application in protoplast production. World J Microb Biot 21: 1611-1616.

Darling ACE, Mau B, Blattner FR \& Perna NT (2004) Mauve: Multiple alignment of conserved genomic sequence with rearrangements. Genome Research 14: 13941403.

De Boer W, Gunnewiek PJAK, Kowalchuk GA \& Van Veen JA (2001) Growth of chitinolytic dune soil beta-subclass Proteobacteria in response to invading fungal hyphae. Appl Environ Microb 67: 3358-3362.

De Boer W, Klein Gunnewiek PJA, Lafeber P, Janse JD, Spit BE \& Woldendorp JW (1998) Anti-fungal properties of chitinolytic dune soil bacteria. Soil Biology and Biochemistry 30: 193-203.

de Boer W, Leveau JH, Kowalchuk GA, Klein Gunnewiek PJ, Abeln EC, Figge MJ, Sjollema K, Janse JD \& van Veen JA (2004) Collimonas fungivorans gen. nov., sp. nov., a chitinolytic soil bacterium with the ability to grow on living fungal hyphae. Int J Syst Evol Microbiol 54: 857-864.

de Bruijn I \& Raaijmakers JM (2009) Regulation of cyclic lipopeptide biosynthesis in Pseudomonas fluorescens by the ClpP protease. J Bacteriol 191: 1910-1923.

De Vrieze M, Pandey P, Bucheli TD, Varadarajan AR, Ahrens CH, Weisskopf L \& Bailly A (2015) Volatile Organic Compounds from Native Potato-associated Pseudomonas as Potential Anti-oomycete Agents. Frontiers in microbiology 6: 1295.

Degenhardt J, Kollner TG \& Gershenzon J (2009) Monoterpene and sesquiterpene synthases and the origin of terpene skeletal diversity in plants. Phytochemistry 70: 1621-1637.

Demain AL \& Fang A (2000) The natural functions of secondary metabolites. Advances in biochemical engineering/biotechnology 69: 1-39.

DeShazer D, Brett PJ, Burtnick MN \& Woods DE (1999) Molecular characterization of genetic loci required for secretion of exoproducts in Burkholderia pseudomallei. Journal of Bacteriology 181: 4661-4664.

Dhillon BK, Chiu TA, Laird MR, Langille MGI \& Brinkman FSL (2013) IslandViewer update: improved genomic island discovery and visualization. Nucleic Acids Research 
Diaz-Sanchez V, Avalos J \& Limon MC (2012) Identification and regulation of fusA, the polyketide synthase gene responsible for fusarin production in Fusarium fujikuroi. Appl Environ Microbiol 78: 7258-7266.

Dicke M \& Sabelis MW (1988) Infochemical Terminology: Based on Cost-Benefit Analysis Rather than Origin of Compounds? Functional Ecology 2: 131-139.

Dickschat JS (2016) Bacterial terpene cyclases. Nat Prod Rep 33: 87-110.

Dickschat JS (2017) Fungal volatiles - a survey from edible mushrooms to moulds. Nat Prod Rep.

Domik D, Magnus N \& Piechulla B (2016) Analysis of a new cluster of genes involved in the synthesis of the unique volatile organic compound sodorifen of Serratia plymuthica 4Rx13. FEMS Microbiol Lett 363.

Dubey MK, Jensen DF \& Karlsson M (2014) An ATP-binding cassette pleiotropic drug transporter protein is required for xenobiotic tolerance and antagonism in the fungal biocontrol agent Clonostachys rosea. Mol Plant Microbe Interact 27: 725-732.

Durand E, Bernadac A, Ball G, Lazdunski A, Sturgis JN \& Filloux A (2003) Type II protein secretion in Pseudomonas aeruginosa: the pseudopilus is a multifibrillar and adhesive structure. J Bacteriol 185: 2749-2758.

\section{$\mathbf{E}$}

Ebel R (2010) Terpenes from marine-derived fungi. Mar Drugs 8: 2340-2368.

Effmert U, Kalderas J, Warnke R \& Piechulla B (2012) Volatile mediated interactions between bacteria and fungi in the soil. J Chem Ecol 38: 665-703.

Epand RF, Pollard JE, Wright JO, Savage PB \& Epand RM (2010) Depolarization, bacterial membrane composition, and the antimicrobial action of ceragenins. Antimicrob Agents Chemother 54: 3708-3713.

Farrand SK, Qin YP \& Oger P (2002) Quorum-sensing system of Agrobacterium plasmids: analysis and utility. Method Enzymol 358: 452-484. 


\section{$\mathbf{F}$}

Felsenstein J (1985) Phylogenies and the comparative method. Am Nat 125: 1-15.

Fleischer J, Breer H \& Strotmann J (2009) Mammalian Olfactory Receptors. Frontiers in Cellular Neuroscience 3: 9.

Franken ACW, Lokman BC, Ram AFJ, van den Hondel CAMJJ, de Weert S \& Punt PJ (2012) Analysis of the role of the Aspergillus niger aminolevulinic acid synthase (hemA) gene illustrates the difference between regulation of yeast and fungal haemand sirohaem-dependent pathways. Fems Microbiology Letters 335: 104-112.

Fritsche K, de Boer W, Gerards S, van den Berg M, van Veen JA \& Leveau JH (2008) Identification and characterization of genes underlying chitinolysis in Collimonas fungivorans Ter331. FEMS Microbiol Ecol 66: 123-135.

Fritsche K, van den Berg M, de Boer W, van Beek TA, Raaijmakers JM, van Veen JA \& Leveau JH (2014) Biosynthetic genes and activity spectrum of antifungal polyynes from Collimonas fungivorans Ter331. Environ Microbiol 16: 1334-1345.

\section{G}

Galan JE \& Collmer A (1999) Type III secretion machines: bacterial devices for protein delivery into host cells. Science 284: 1322-1328.

Galardini M, Biondi EG, Bazzicalupo M \& Mengoni A (2011) CONTIGuator: a bacterial genomes finishing tool for structural insights on draft genomes. Source Code for Biology and Medicine 6: 11.

Galens K, Orvis J, Daugherty S, Creasy HH, Angiuoli S, White O, Wortman J, Mahurkar A \& Giglio MG (2011) The IGS standard operating procedure for automated prokaryotic annotation. Stand Genomic Sci 4: 244-251.

Gallucci MN, Oliva M, Casero C, Dambolena J, Luna A, Zygadlo J \& Demo M (2009) Antimicrobial combined action of terpenes against the food-borne microorganisms Escherichia coli, Staphylococcus aureus and Bacillus cereus. Flavour Frag J 24: 348-354.

Garbeva P, Hordijk C, Gerards S \& de Boer W (2014) Volatile-mediated interactions between phylogenetically different soil bacteria. Frontiers in microbiology 5: 289.

Gottesman S (2003) Proteolysis in bacterial regulatory circuits. Annu Rev Cell Dev Bi 19: 565-587. 
Grigoriev IV, Nikitin R, Haridas S, et al. (2014) MycoCosm portal: gearing up for 1000 fungal genomes. Nucleic Acids Res 42: D699-704.

Grissa I, Vergnaud G \& Pourcel C (2007) CRISPRFinder: a web tool to identify clustered regularly interspaced short palindromic repeats. Nucleic Acids Research 35: W52-W57.

Gurtler H, Pedersen R, Anthoni U, Christophersen C, Nielsen PH, Wellington EM, Pedersen C \& Bock K (1994) Albaflavenone, a sesquiterpene ketone with a zizaene skeleton produced by a streptomycete with a new rope morphology. The Journal of antibiotics 47: 434-439.

\section{H}

Hacquard S (2017) Commentary: Microbial Small Talk: Volatiles in Fungal-Bacterial Interactions. Frontiers in microbiology 8.

Hakvag S, Fjaervik E, Klinkenberg G, Borgos SE, Josefsen KD, Ellingsen TE \& Zotchev SB (2009) Violacein-producing Collimonas sp. from the sea surface microlayer of costal waters in Trondelag, Norway. Mar Drugs 7: 576-588.

Hashizume H, Igarashi M, Hattori S, Hori M, Hamada M \& Takeuchi T (2001) Tripropeptins, novel antimicrobial agents produced by Lysobacter sp. I. Taxonomy, isolation and biological activities. Journal of Antibiotics 54: 1054-1059.

Hashizume H, Hirosawa S, Sawa R, Muraoka Y, Ikeda D, Naganawa H \& Igarashi M (2004) Tripropeptins, novel antimicrobial agents produced by Lysobacter sp - II. Structure elucidation. Journal of Antibiotics 57: 52-58.

Heil M (2014) Herbivore-induced plant volatiles: targets, perception and unanswered questions. New Phytologist 204: 297-306.

Hengge R \& Bukau B (2003) Proteolysis in prokaryotes: protein quality control and regulatory principles. Molecular Microbiology 49: 1451-1462.

Hestbjerg H, Nielsen KF, Thrane U \& Elmholt S (2002) Production of trichothecenes and other secondary metabolites by Fusarium culmorum and Fusarium equiseti on common laboratory media and a soil organic matter agar: an ecological interpretation. J Agric Food Chem 50: 7593-7599.

Hider RC \& Kong XL (2010) Chemistry and biology of siderophores. Natural Product Reports 27: 637-657. 
Hohn TM \& Beremand PD (1989) Isolation and nucleotide sequence of a sesquiterpene cyclase gene from the trichothecene-producing fungus Fusarium sporotrichioides. Gene 79: 131-138.

Holden MTG, Seth-Smith HMB, Crossman LC, et al. (2009) The genome of Burkholderia cenocepacia J2315, an epidemic pathogen of cystic fibrosis patients (vol 191, pg 261, 2009). Journal of Bacteriology 191: 2907-2907.

Holt C \& Yandell M (2011) MAKER2: an annotation pipeline and genome-database management tool for second-generation genome projects. BMC Bioinformatics 12: 491.

Hoppener-Ogawa S, Leveau JH, Hundscheid MP, van Veen JA \& de Boer W (2009) Impact of Collimonas bacteria on community composition of soil fungi. Environ Microbiol 11: 1444-1452.

Hoppener-Ogawa S, de Boer W, Leveau JH, van Veen JA, de Brandt E, Vanlaere E, Sutton H, Dare DJ \& Vandamme P (2008) Collimonas arenae sp. nov. and Collimonas pratensis sp. nov., isolated from (semi-)natural grassland soils. Int J Syst Evol Microbiol 58: 414-419.

Huang Y, Niu BF, Gao Y, Fu LM \& Li WZ (2010) CD-HIT Suite: a web server for clustering and comparing biological sequences. Bioinformatics 26: 680-682.

Jackson RW, Preston GM \& Rainey PB (2005) Genetic characterization of Pseudomonas fluorescens SBW25 rsp gene expression in the phytosphere and in vitro. Journal of Bacteriology 187: 8477-8488.

Jenal U \& Hengge-Aronis R (2003) Regulation by proteolysis in bacterial cells. Curr Opin Microbiol 6: 163-172.

Jongedijk E, Cankar K, Ranzijn J, van der Krol S, Bouwmeester H \& Beekwilder J (2015) Capturing of the monoterpene olefin limonene produced in Saccharomyces cerevisiae. Yeast 32: 159-171.

\section{K}

Kanchiswamy CN, Malnoy M \& Maffei ME (2015) Bioprospecting bacterial and fungal volatiles for sustainable agriculture. Trends in plant science 20: 206-211. 
Keller NP (2015) Translating biosynthetic gene clusters into fungal armor and weaponry. Nat Chem Biol 11: 671-677.

Keller NP, Turner G \& Bennett JW (2005) Fungal secondary metabolism - From biochemistry to genomics. Nature Reviews Microbiology 3: 937-947.

Kim YT, Lee YR, Jin J, Han KH, Kim H, Kim JC, Lee T, Yun SH \& Lee YW (2005) Two different polyketide synthase genes are required for synthesis of zearalenone in Gibberella zeae. Mol Microbiol 58: 1102-1113.

King R, Urban M, Hammond-Kosack MC, Hassani-Pak K \& Hammond-Kosack KE (2015) The completed genome sequence of the pathogenic ascomycete fungus Fusarium graminearum. BMC Genomics 16: 544.

Knudsen IMB, Hockenhull J \& Jensen DF (1995) Biocontrol of seedling diseases of barley and wheat caused by Fusarium culmorum and Bipolaris sorokiniana: effects of selected fungal antagonists on growth and yield components. Plant Pathology 44: 467-477.

Kobayashi DY, Reedy RM, Bick J \& Oudemans PV (2002) Characterization of a chitinase gene from Stenotrophomonas maltophilia strain 34S1 and its involvement in biological control. Appl Environ Microb 68: 1047-1054.

Korf I (2004) Gene finding in novel genomes. BMC Bioinformatics 5.

Kovac J, Simunovic K, Wu Z, Klancnik A, Bucar F, Zhang Q \& Mozina SS (2015) Antibiotic resistance modulation and modes of action of (-)-alpha-pinene in Campylobacter jejuni. PLoS One 10: e0122871.

Krzywinski M, Schein J, Birol I, Connors J, Gascoyne R, Horsman D, Jones SJ \& Marra MA (2009) Circos: An information aesthetic for comparative genomics. Genome Research 19: 1639-1645.

Langille MGI \& Brinkman FSL (2009) IslandViewer: an integrated interface for computational identification and visualization of genomic islands. Bioinformatics 25: 664-665.

Lauchli R, Pitzer J, Kitto RZ, Kalbarczyk KZ \& Rabe KS (2014) Improved selectivity of an engineered multi-product terpene synthase. Org Biomol Chem 12: 4013-4020.

Leveau JH, Uroz S \& de Boer W (2010) The bacterial genus Collimonas: mycophagy, 
weathering and other adaptive solutions to life in oligotrophic soil environments. Environ Microbiol 12: 281-292.

Lewenza S, Conway B, Greenberg EP \& Sokol PA (1999) Quorum sensing in Burkholderia cepacia: identification of the LuxRI homologs CepRI. Journal of Bacteriology 181: 748-756.

Li J, Yao Y, Xu HH, Hao L, Deng Z, Rajakumar K \& Ou HY (2015) SecReT6: a web-based resource for type VI secretion systems found in bacteria. Environ Microbiol.

Lou LL, Qian GL, Xie YX, et al. (2011) Biosynthesis of HSAF, a tetramic acid-containing macrolactam from Lysobacter enzymogenes. Journal of the American Chemical Society 133: 643-645.

\section{M}

Maffei M, Camusso W \& Sacco S (2001) Effect of Mentha x piperita essential oil and monoterpenes on cucumber root membrane potential. Phytochemistry 58: 703-707.

Maffei ME, Mithofer A \& Boland W (2007) Before gene expression: early events in plant-insect interaction. Trends in plant science 12: 310-316.

McClean KH, Winson MK, Fish L, et al. (1997) Quorum sensing and Chromobacterium violaceum: exploitation of violacein production and inhibition for the detection of $\mathrm{N}$-acylhomoserine lactones. Microbiol-Uk 143: 3703-3711.

McCormick SP, Alexander NJ \& Harris LJ (2010) CLM1 of Fusarium graminearum encodes a longiborneol synthase required for culmorin production. App/ Environ Microbiol 76: 136-141.

Medema MH, Paalvast Y, Nguyen DD, Melnik A, Dorrestein PC, Takano E \& Breitling R (2014) Pep2Path: automated mass spectrometry-guided genome mining of peptidic natural products. Plos Comput Biol 10.

Medema MH, Blin K, Cimermancic P, de Jager V, Zakrzewski P, Fischbach MA, Weber T, Takano E \& Breitling R (2011) antiSMASH: rapid identification, annotation and analysis of secondary metabolite biosynthesis gene clusters in bacterial and fungal genome sequences. Nucleic Acids Res 39: W339-346.

Mela F, Fritsche K, de Boer W, van Veen JA, de Graaff LH, van den Berg M \& Leveau $J H$ (2011) Dual transcriptional profiling of a bacterial/fungal confrontation: Collimonas fungivorans versus Aspergillus niger. ISME J 5: 1494-1504. 
Mela F, Fritsche K, de Boer W, van den Berg M, van Veen JA, Maharaj NN \& Leveau $J \mathrm{H}$ (2012) Comparative genomics of bacteria from the genus Collimonas: linking (dis) similarities in gene content to phenotypic variation and conservation. Environ Microbiol Rep 4: 424-432.

Mela F, Fritsche K, Boersma H, van Elsas JD, Bartels D, Meyer F, de Boer W, van Veen JA \& Leveau JH (2008) Comparative genomics of the pIPO2/pSB102 family of environmental plasmids: sequence, evolution, and ecology of pTer331 isolated from Collimonas fungivorans Ter331. FEMS Microbiol Ecol 66: 45-62.

Morath SU, Hung R \& Bennett JW (2012) Fungal volatile organic compounds: A review with emphasis on their biotechnological potential. Fungal Biology Reviews 26: 73-83.

\section{$\mathbf{N}$}

Nazzaro F, Fratianni F, De Martino L, Coppola R \& De Feo V (2013) Effect of Essential Oils on Pathogenic Bacteria. Pharmaceuticals 6: 1451-1474.

Nika JR, Latimer JL, Ward CK, Blick RJ, Wagner NJ, Cope LD, Mahairas GG, Munson RS, Jr. \& Hansen EJ (2002) Haemophilus ducreyi requires the flp gene cluster for microcolony formation in vitro. Infection and immunity 70: 2965-2975.

\section{0}

Opelt K \& Berg G (2004) Diversity and antagonistic potential of bacteria associated with bryophytes from nutrient-poor habitats of the Baltic Sea coast. App/ Environ Microb 70: 6569-6579.

\section{$\mathbf{P}$}

Page MJ \& Di Cera E (2008) Serine peptidases: classification, structure and function. Cell Mol Life Sci 65: 1220-1236.

Parra G, Bradnam K \& Korf I (2007) CEGMA: a pipeline to accurately annotate core genes in eukaryotic genomes. Bioinformatics 23.

Pasquali M, Beyer M, Logrieco A, et al. (2016) A European Database of Fusarium graminearum and F. culmorum Trichothecene Genotypes. Frontiers in microbiology 7: 406 . 
Planet PJ, Kachlany SC, Fine DH, DeSalle R \& Figurski DH (2003) The widespread colonization island of Actinobacillus actinomycetemcomitans. Nature genetics 34: 193198.

Proctor RH \& Hohn TM (1993) Aristolochene synthase. Isolation, characterization, and bacterial expression of a sesquiterpenoid biosynthetic gene (Ari1) from Penicillium roqueforti. J Biol Chem 268: 4543-4548.

\section{Q}

Quin MB, Flynn CM \& Schmidt-Dannert C (2014) Traversing the fungal terpenome. Natural product reports 31: 1449-1473.

\section{$\mathbf{R}$}

Raaijmakers JM \& Mazzola M (2012) Diversity and natural functions of antibiotics produced by beneficial and plant pathogenic bacteria. Annual Review of Phytopathology, Vol 50 50: 403-424.

Raaijmakers JM, de Bruijn I \& de Kock MJD (2006) Cyclic lipopeptide production by plant-associated Pseudomonas spp.: Diversity, activity, biosynthesis, and regulation. Mol Plant Microbe In 19: 699-710.

Raaijmakers JM, de Bruijn I, Nybroe O \& Ongena M (2010) Natural functions of lipopeptides from Bacillus and Pseudomonas: more than surfactants and antibiotics. FEMS microbiology reviews 34: 1037-1062.

Rabe P \& Dickschat JS (2013) Rapid Chemical Characterization of Bacterial Terpene Synthases. Angewandte Chemie International Edition 52: 1810-1812.

Rabe P, Citron CA \& Dickschat JS (2013) Volatile terpenes from actinomycetes: a biosynthetic study correlating chemical analyses to genome data. Chembiochem : a European journal of chemical biology 14: 2345-2354.

Rabea El, Badawy MET, Stevens CV, Smagghe G \& Steurbaut W (2003) Chitosan as antimicrobial agent: Applications and mode of action. Biomacromolecules 4: 14571465.

Rasmann S, Kollner TG, Degenhardt J, Hiltpold I, Toepfer S, Kuhlmann U, Gershenzon J \& Turlings TC (2005) Recruitment of entomopathogenic nematodes by insect-damaged maize roots. Nature 434: 732-737. 
Rawlings ND, Waller M, Barrett AJ \& Bateman A (2014) MEROPS: the database of proteolytic enzymes, their substrates and inhibitors. Nucleic Acids Res 42: D503-509.

Records AR (2011) The type VI secretion system: a multipurpose delivery system with a phage-like machinery. Mol Plant Microbe In 24: 751-757.

Rocha O, Ansari K \& Doohan FM (2005) Effects of trichothecene mycotoxins on eukaryotic cells: a review. Food additives and contaminants 22: 369-378.

Rottig M, Medema MH, Blin K, Weber T, Rausch C \& Kohlbacher O (2011) NRPSpredictor2-a web server for predicting NRPS adenylation domain specificity. Nucleic Acids Research 39: W362-W367.

Rudnick M-B (2015) Mycophagous soil bacteria. 87-124.

Rugbjerg P, Naesby M, Mortensen UH \& Frandsen RJ (2013) Reconstruction of the biosynthetic pathway for the core fungal polyketide scaffold rubrofusarin in Saccharomyces cerevisiae. Microbial cell factories 12: 31.

Russell AB, Hood RD, Bui NK, LeRoux M, Vollmer W \& Mougous JD (2011) Type VI secretion delivers bacteriolytic effectors to target cells. Nature 475: 343-U392.

Ryu CM, Farag MA, Hu CH, Reddy MS, Wei HX, Pare PW \& Kloepper JW (2003) Bacterial volatiles promote growth in Arabidopsis. Proc Natl Acad Sci U S A 100: 4927-4932.

\section{S}

Saitou N \& Nei M (1987) The neighbor-joining method - a new method for reconstructing phylogenetic trees. Mol Biol Evol 4: 406-425.

Sato K \& Touhara K (2009) Insect olfaction: receptors, signal transduction, and behavior. Results and problems in cell differentiation 47: 121-138.

Schalk IJ, Yue WW \& Buchanan SK (2004) Recognition of iron-free siderophores by TonB-dependent iron transporters. Molecular Microbiology 54: 14-22.

Scherm B, Balmas V, Spanu F, Pani G, Delogu G, Pasquali M \& Migheli Q (2013) Fusarium culmorum: causal agent of foot and root rot and head blight on wheat. Molecular plant pathology 14: 323-341.

Scherm B, Orru M, Balmas V, Spanu F, Azara E, Delogu G, Hammond TM, Keller NP \& Migheli Q (2011) Altered trichothecene biosynthesis in TRI6-silenced transformants of Fusarium culmorum influences the severity of crown and foot rot on 
durum wheat seedlings. Molecular plant pathology 12: 759-771.

Schmidt R, Cordovez V, de Boer W, Raaijmakers J \& Garbeva P (2015) Volatile affairs in microbial interactions. ISME J 9: 2329-2335.

Schmidt R, Etalo DW, de Jager V, Gerards S, Zweers H, de Boer W \& Garbeva P (2015) Microbial Small Talk: Volatiles in Fungal-Bacterial Interactions. Frontiers in microbiology 6: 1495.

Schmidt R, Jager V, Zuhlke D, et al. (2017) Fungal volatile compounds induce production of the secondary metabolite Sodorifen in Serratia plymuthica PRI-2C. Sci Rep 7: 862.

Schmiel DH \& Miller VL (1999) Bacterial phospholipases and pathogenesis. Microbes Infect 1: 1103-1112.

Schulz-Bohm K, Geisen S, Wubs ER, Song C, de Boer W \& Garbeva P (2017) The prey's scent - Volatile organic compound mediated interactions between soil bacteria and their protist predators. ISME J11: 817-820.

Schwarz S, Hood RD \& Mougous JD (2010) What is type VI secretion doing in all those bugs? Trends in microbiology 18: 531-537.

Schwyn B \& Neilands JB (1987) Universal chemical-assay for the detection and determination of siderophores. Anal Biochem 160: 47-56.

Sharifi R \& Ryu CM (2016) Are Bacterial Volatile Compounds Poisonous Odors to a Fungal Pathogen Botrytis cinerea, Alarm Signals to Arabidopsis Seedlings for Eliciting Induced Resistance, or Both? Frontiers in microbiology 7: 196.

Sherif M, Becker EM, Herrfurth C, Feussner I, Karlovsky P \& Splivallo R (2016) Volatiles Emitted from Maize Ears Simultaneously Infected with Two Fusarium Species Mirror the Most Competitive Fungal Pathogen. Frontiers in plant science 7: 1460.

Simão FA, Waterhouse RM, loannidis P, Kriventseva EV \& Zdobnov EM (2015) BUSCO: assessing genome assembly and annotation completeness with single-copy orthologs. Bioinformatics 31: 3210-3212.

Singh SK, Strobel GA, Knighton B, Geary B, Sears I \& Ezra D (2011) An endophytic Phomopsis sp. possessing bioactivity and fuel potential with its volatile organic compounds. Microbial ecology 61: 729-739.

Snel B, Bork P \& Huynen MA (2002) The identification of functional modules from the genomic association of genes. P Natl Acad Sci USA 99: 5890-5895. 
Stamatakis A (2014) RAxML version 8: a tool for phylogenetic analysis and post-analysis of large phylogenies. Bioinformatics 30: 1312-1313.

Stanke M \& Waack S (2003) Gene prediction with a hidden Markov model and a new intron submodel. Bioinformatics 19.

Stock AM, Robinson VL \& Goudreau PN (2000) Two-component signal transduction. Annu Rev Biochem 69: 183-215.

\section{$\mathbf{T}$}

Tamura K, Nei M \& Kumar S (2004) Prospects for inferring very large phylogenies by using the neighbor-joining method. P Natl Acad Sci USA 101: 11030-11035.

Tamura K, Stecher G, Peterson D, Filipski A \& Kumar S (2013) MEGA6: molecular evolutionary genetics analysis version 6.0. Mol Biol Evol 30: 2725-2729.

Ter-Hovhannisyan V, Lomsadze A, Chernoff YO \& Borodovsky M (2008) Gene prediction in novel fungal genomes using an ab initio algorithm with unsupervised training. Genome Res 18: 1979-1990.

Tettelin H, Masignani V, Cieslewicz MJ, et al. (2005) Genome analysis of multiple pathogenic isolates of Streptococcus agalactiae: implications for the microbial "pan-genome". Proc Natl Acad Sci U S A 102: 13950-13955.

Tevell Åberg A, Solyakov A \& Bondesson U (2013) Development and in-house validation of an LC-MS/MS method for the quantification of the mycotoxins deoxynivalenol, zearalenone, T-2 and HT-2 toxin, ochratoxin A and fumonisin B1 and B2 in vegetable animal feed. Food Additives \& Contaminants: Part A 30: 541-549.

Tholl D (2006) Terpene synthases and the regulation, diversity and biological roles of terpene metabolism. Current opinion in plant biology 9: 297-304.

Tomich M, Planet PJ \& Figurski DH (2007) The tad locus: postcards from the widespread colonization island. Nat Rev Microbio/ 5: 363-375.

Trapp SC \& Croteau RB (2001) Genomic organization of plant terpene synthases and molecular evolutionary implications. Genetics 158: 811-832.

Trombetta D, Castelli F, Sarpietro MG, Venuti V, Cristani M, Daniele C, Saija A, Mazzanti G \& Bisignano G (2005) Mechanisms of Antibacterial Action of Three Monoterpenes. Antimicrobial Agents and Chemotherapy 49: 2474-2478. 
Tseng TT, Tyler BM \& Setubal JC (2009) Protein secretion systems in bacterial-host associations, and their description in the gene ontology. Bmc Microbio/ 9.

Tyc O, van den Berg M, Gerards S, van Veen JA, Raaijmakers JM, de Boer W \& Garbeva P (2014) Impact of interspecific interactions on antimicrobial activity among soil bacteria. Frontiers in microbiology 5: 567.

\section{$\mathbf{U}$}

Ulrich LE, Koonin EV \& Zhulin IB (2005) One-component systems dominate signal transduction in prokaryotes. Trends in microbiology 13: 52-56.

\section{V}

Van Tol RWHM, Van Der Sommen ATC, Boff MIC, Van Bezooijen J, Sabelis MW \& Smits PH (2001) Plants protect their roots by alerting the enemies of grubs. Ecology letters 4: 292-294.

Vespermann A, Kai M \& Piechulla B (2007) Rhizobacterial volatiles affect the growth of fungi and Arabidopsis thaliana. Appl Environ Microbiol 73: 5639-5641.

\section{W}

Wagacha JM \& Muthomi JW (2007) Fusarium culmorum: Infection process, mechanisms of mycotoxin production and their role in pathogenesis in wheat. Crop Protection 26: 877-885.

Wang YM, Liu JB \& Peng SQ (2009) Effects of Fusarium mycotoxin butenolide on myocardial mitochondria in vitro. Toxicology mechanisms and methods 19: 79-85.

Ward TJ, Bielawski JP, Kistler HC, Sullivan E \& O'Donnell K (2002) Ancestral polymorphism and adaptive evolution in the trichothecene mycotoxin gene cluster of phytopathogenic Fusarium. Proc Natl Acad Sci U S A 99: 9278-9283.

Warmink JA \& van Elsas JD (2008) Selection of bacterial populations in the mycosphere of Laccaria proxima: is type III secretion involved? Isme Journal 2: 887-900.

Wu JJ, de Jager VC, Deng WL \& Leveau JH (2015) Finished genome sequence of Collimonas arenae Cal35. Genome announcements 3. 


\section{$\mathbf{Y}$}

Yamada Y, Kuzuyama T, Komatsu M, Shin-Ya K, Omura S, Cane DE \& Ikeda H (2015) Terpene synthases are widely distributed in bacteria. Proc Natl Acad Sci U S A 112: 857-862.

Yoshikuni Y, Martin VJ, Ferrin TE \& Keasling JD (2006) Engineering cotton (+)-delta-cadinene synthase to an altered function: germacrene D-4-ol synthase. Chemistry \& biology 13: 91-98.

Yu FG, Zaleta-Rivera K, Zhu XC, Huffman J, Millet JC, Harris SD, Yuen G, Li XC \& Du LC (2007) Structure and biosynthesis of heat-stable antifungal factor (HSAF), a broad-spectrum antimycotic with a novel mode of action. Antimicrobial Agents and Chemotherapy 51: 64-72.

\section{$\mathbf{Z}$}

Zhao B, Lin X, Lei L, Lamb DC, Kelly SL, Waterman MR \& Cane DE (2008) Biosynthesis of the sesquiterpene antibiotic albaflavenone in Streptomyces coelicolor A3(2). Journal of Biological Chemistry 283: 8183-8189.

Zhou Y, Liang YJ, Lynch KH, Dennis JJ \& Wishart DS (2011) PHAST: a fast phage search tool. Nucleic Acids Research 39: W347-W352. 



\section{/ Summary}

/ Zusammenfassung

/ Acknowledgement

/ Curriculum vitae

/ Education Statement 



\section{Summary}

Microorganisms, such as bacteria and fungi produce odors, also known as volatile organic compounds (VOCs). VOCs are small, carbon-containing molecules that exhibit high vapor pressure under ambient conditions and have low boiling points. Especially in soil and in the rhizosphere, these unique properties provide the advantage to travel over long distances, mediating interaction and communication amongst physically separated microorganisms. In this thesis, we zoom for the first time into the mechanisms of volatile mediated fungalbacterial interactions. For this, in vitro bioassays were combined with "omic" approaches, such as genomics, transcriptomics, proteomics and metabolomics.

Whilst so far most attention has been paid to bacterial VOCs, the first step of the thesis was to investigate the diversity and ecological role of fungal VOCs. Comparing the "volatilome" of a range of soil/rhizospheric fungi and oomycete revealed that each microbe has its own chemical signature and that the growth stage and the nutritional status (rich vs. poor media) have a strong effect on VOC emission. This makes it tempting to speculate that filamentous microbes adjust the production of VOCs for interactions and communication with other microbes depending on the nutrient status. Linking the individual "volatilomes" with their effects on the behavior of bacteria showed that in particular terpene volatiles of the fungus F. culmorum affect the motility of two bacterial isolates, Collimonas pratensis and Serratia plymuthica.

Curiosity about the underlying mechanisms of VOC perception and responses lead to the next step in which we analyzed the transcriptome and proteome of S. plymuthica when exposed to VOCs emitted by the fungal plant pathogen F. culmorum. We found that the bacterium responds to fungal VOCs with changes in gene and protein expression related to motility, signal transduction, energy metabolism, cell envelope biogenesis, and secondary metabolite production. Metabolomic analysis of the bacterium exposed to the fungal VOCs and heterologous co-expression of a terpene synthase and a methyltransferase revealed the production of the unusual terpene sodorifen in response to fungal VOCs. These results lead to the proposition that terpenes are a lingua franca in long-distance communication between bacteria and fungi. However, it remains to be elucidated in future studies what the ecological roles of sodorifen are in respect of the context of microbe-microbe and microbe-plant interactions. 
Intrigued by the newly discovered "language", we went on a quest for more secret terpene producers in the belowground world by comparing the genomes of several Collimonas species. In this way we identified two terpene synthase genes that encode for the production of germacrene D-4-ol by, and several sesquiterpens, including $\delta$-cadinene. Not only bacteria are a rich source of terpenes, but fungi are promising producers too. Thus, we sequenced the fungal genome of our previous studies, Fusarium culmorum, to look for genes encoding for terpenes and other secondary metabolites that play important roles in fungal pathogenicity and in the interaction with other microorganisms. Searching through the genome we identified two terpene synthase genes, longiborneol synthase and trichodiene synthase that encode for the production of a whole array of terpenes. We propose that there may be many new, undiscovered terpenes hiding in the genomes of bacteria and fungi. However, also this quest remains to be continued in future studies.

The obtained results of this thesis set the basis for further understanding the natural roles of terpene-mediated microbe-microbe as well as microbe-plant interactions. Ultimately, this knowledge can be translated into innovative strategies for developments towards ecofriendly and sustainable agricultural practices. 


\section{Zusammenfassung}

Mikroorganismen, wie Bakterien und Pilze, erzeugen Gerüche, die auch als flüchtige organische Verbindungen (VOCs) bekannt sind. VOCs sind kleine, kohlenstoffhaltige Moleküle, die unter Umgebungsbedingungen einen hohen Dampfdruck und niedrige Siedepunkte aufweisen. Besonders im Boden und in der Rhizosphäre bieten diese einzigartigen Eigenschaften den Vorteil lange Distanzen zu überwinden und somit die Interaktion und Kommunikation zwischen physisch getrennten Mikroorganismen zu vermitteln. In dieser Arbeit zoomen wir zum ersten Mal in die unterliegenden Mechanismen dieser flüchtigen Pilz-BakterienInteraktion. Dazu kombinierten wir in vitro Bioassays mit "omik" Ansätzen, wie Genomik, Transkriptomik, Proteomik und Metabolomik.

Während bislang die meiste Aufmerksamkeit auf bakterielle VOCs gelegt wurde, war der erste Schritt der Arbeit, die Vielfalt und die ökologische Rolle von Pilz-VOCs zu untersuchen. Der Vergleich der "Volatilome", einer Reihe von Boden / Rhizosphärenpilzen und Oomycete, ergab, dass jede Mikrobe ihre eigene chemische Signatur hat und dass die Wachstumsstufe und der Ernährungszustand (reich vs. nährstoffarme Medien) einen starken Einfluss auf die VOC-Emission haben. Dies veranlasst zu spekulieren, dass filamentöse Mikroben die Produktion von VOCs für Interaktionen und die Kommunikation mit anderen Mikroben je nach Nährstoffstatus anpassen. Die Verknüpfung der einzelnen "Volatilomen" mit ihren Auswirkungen auf das Verhalten von Bakterien zeigte, dass insbesondere terpenflüchtige Stoffe des Pilzes F. culmorum die Motilität von zwei Bakterienisolaten, Collimonas pratensis und Serratia plymuthica beeinflussen.

Die Neugier über die zugrundeliegenden Mechanismen der VOCInteraktionen führte zu dem nächsten Schritt, in dem wir das Transkriptom und das Proteom von S. plymuthica analysierten. Hierzu wurde das Bakterium VOCs des Pilzpflanzenpathogen F. culmorum ausgesetzt. Wir fanden heraus, dass das Bakterium auf Pilz-VOCs mit Veränderungen in der Gen- und Protein-Expression reagiert, die sich auf Motilität, Signaltransduktion, Energiestoffwechsel, Zellhülle Biogenese und sekundäre Metabolitproduktion beziehen. Die metabolomische Analyse des Bakteriums, das den Pilz-VOCs ausgesetzt war und heterologen Co-Expression einer Terpen-Synthase und einer Methyltransferase, zeigte die Produktion des ungewöhnlichen Terpen Sodorifen als Reaktion auf Pilz-VOCs. 
Diese Ergebnisse führen zu der Hypothese, dass Terpene eine lingua franca in der Fernkommunikation zwischen Bakterien und Pilzen sind. Allerdings bleibt in künftigen Studien zu klären, welche ökologischen Rollen Sodorifen im Kontext von Mikroben-Mikroben- und Mikroben-Pflanzen-Wechselwirkungen spielen.

Fasziniert von der neu entdeckten "Sprache", gingen wir auf die Suche nach mehr geheimen Terpenproduzenten in der Bodenwelt, indem wir die Genome von mehreren Collimonas-Arten verglichen. Auf diese Weise konnten wir zwei TerpenSynthase-Gene identifizieren, die für die Herstellung von Germacren-D-4-ol und mehreren Sesquiterpenen, einschließlich $\delta$-Cadinen, kodierten. Nicht nur Bakterien sind eine reiche Quelle von Terpenen, sondern auch Pilze sind vielversprechende Produzenten. Folglich sequenzierten wir das Pilzgenom unserer früheren Studien, Fusarium culmorum, um nach Genen zu suchen, die für Terpene und andere sekundäre Metaboliten kodieren und eine wichtige Rolle in der Pilzpathogenität und in der Interaktion mit anderen Mikroorganismen spielen. In dem Genom wurden zwei Terpen-Synthase-Gene, Longiborneolsynthase und Trichodien-Synthase identifiziert, die für die Herstellung einer ganzen Reihe von Terpenen kodieren. Unser Ergebnis ist, dass es viele neue unentdeckte Terpene gibt, die sich in den Genomen von Bakterien und Pilzen verstecken. Allerdings ist dies eine Arbeit und Suche, die nur durch zukünftigen Studien erbracht werden könnte.

Die gewonnenen Ergebnisse dieser Arbeit bilden die Grundlage für das weitere Verständnis der ökologischen Rollen von Terpen-vermittelten MikrobenMikroben sowie Mikroben-Pflanzen-Interaktionen. Letztlich kann dieses Wissen in innovative Strategien für die Entwicklungen von umweltfreundlichen und nachhaltigen landwirtschaftlichen Praktiken umgesetzt werden. 


\section{Acknowledgement}

I still remember the first visit to the Netherlands and the $\mathrm{NIOO}$ as if it was yesterday. I was very excited and nervous at the same time to move to the country in the north I had associated back then with my cousin who had moved here more than 20 years ago. When looking back at the last 4 and half years it feels like a rich and intense ride during which I met many wonderful people who found a special place in my heart.

First of all I would like to thank Paolina. You were more than just a supervisor. I got to know you on a personal level throughout the last years and I'm incredibly thankful for all the support you gave me. You're a smart, empathetic woman with a kind heart! I learned a lot from you, from being a more organized person to becoming independent as a scientist. On top of that you were giving me the creative freedom to explore other projects outside my PhD.

Wietse. our conversations were very valuable to me. Was it your scientific input or the exchange of our latest music discoveries. I could always knock your door when I had a question or doubt and needed help. Thanks for all your support.

Victor de Jager. without your help my project would have been impossible. You helped me to work through the masses of 'omics' data. Whatever it was you always had an open ear and discussions with you, both scientific and personal, made it fun to work with you. Take care of yourself; I hope the future will treat you with kindness!

Thanks to all my collaborators. I really enjoyed getting to know and working with many people from different universities. Katarina Cankar and Jules Beekwilder, thanks for your help with the sodorifen mutant. Riya Menezes, Aleš Svatoš, Jeroen Dickschat and Lukas Lauterbach, thanks for helping me with the measurements of the secondary metabolites of Fusarium. Magnus Karlsson, Mikael Durling, Erik Nordkvist and Mukesh Dubey, thank you for the help with the Fusarium genome and the mycotoxin work during my stay in Sweden.

One of the most fun summers was our trip to Greifswald with Agaat, Marcelo and Anna. Thank you Kathrin, Daniela, Jörg and Christian and everyone else in the department for all your help and hospitality. Besides working hard in the lab at feelingly $40^{\circ} \mathrm{C}$, we had had a great time on the island of Usedom and inventing our 
own Black Stories after all those experimental failures.

Desalegn. You have undoubtedly a beautiful soul and a unique way of seeing the world! You helped me so much in the beginning of my PhD when I was lost in the sea of metabolomics data. Thanks for fishing me out and teaching me how to swim! I wish you the best of everything life has to offer.

Thank you to all the people I met during my time in Sweden. Les, you were the best office mate, thanks for all the conversations about life! Valerie, Tina, Roman, Lea, Germán, Miguel, Aitzi, Jonás, Mariya - thanks for the fun times. Efstratia and Haris, thanks for your friendship. Isidora, thanks for your support. Vasiliki. I met you in the tunes of Yann Tiersen and since then we lived through many intense moments. Thank you for sharing your philosophy with me and for showing me "how to see rightly".

I was lucky to meet some artists during my PhD whom l'd like to thank for the wonderful work that came out of those projects. Sonja Bäumel, it was a pleasure to work with you and all other people from the fifty percent human consortium and to get an insight into the world of Bioart. Hauke Smidt, Hanne Tygtgat and LooWee Chia, it was fun discussing and working with you in this project.

Eva van Ooij and Lucas Wiegerink. I enjoyed a lot working with you on an arts and science project of microbial music and discussing about interdisciplinarity.

Thanks to my paranymphs. We experienced many fun and creative moments together. Was it our post-crossing addiction, our adventures with the Oogjes family, creative outbursts in the house, or music festivals... It was a wonderful time, which I will always think back to with a smile on my face! You're both very different personalities, though fantastic in your own ways. And that's what made our "three musketeers" team so incredible. Maaike. The beginning of our friendship couldn't have been more adventurous. Stuck on an island in France. Being literally cut off from the rest of the world. Your way of perceiving your surrounding feels like stepping through a hidden door into a magical world. Thanks for showing me this world through your eyes. Marta. You are literally a colorful explosion. You have so much energy to share that immediately infects everyone around you. When thinking of you this quote comes to my mind "The creative adult is the child that survived". Thank you for all the conversations, political activities, and for introducing 
me to your family and life in Spain.

Enric. Our friendship started during our weekly Dutch lessons with Emma. I love your openness and humor. You're a person with whom I can be totally free and express whatever comes to my mind, no matter how crazy or weird it may be. Thanks for always having been there in good and in difficult moments.

Stijn. You are my friend since our first year seminar at $\mathrm{NIOO}$ and you have been there during the whole time of our PhD. I always valued our conversations. You're very straightforward and honest and you always managed to give me a different perspective on things.

Elisabet and Niklas. Thank you for having shared many music and movie moments together and for showing me a part of Sweden.

Maria. You're a smart and engaged woman with whom I shared countless discussions about music, politics and feminism. Thank you for having me provided with so many interesting events and thoughts!

Penny. I met first in Austria and we reconnected when I moved to the Netherlands. I will never forget our crazy adventures with our friend Omar. You're a wonderful person, filled with joy and music!

Anna. Thank you for all the great conversations and dinners. Your music always puts a smile on my face.

João. Even though we haven't lived in the same country since you moved back to Brazil, I always carry you in my heart. What a wonderful reunion we had when I came to Brazil. I won't forget our endless Caipirinha nights and dancing in the streets of Rio. Até mais!

Sam. Thank you for all your beautiful post and for showing me part of your life in the UK.

Thanks to my dear Villa Pomonians. It was a pleasure to meet so many inspiring women and to share a house with you! l'll always remember our dinners, parties and cozy hangouts. You made me feel at home. Lura, Marta, Sara, Bri, Yngrid, Marika, I'll always think back of your words "Life is better weird than boring"! Tuna, my love. You brought so much the sunshine into my life. I admire your positive attitude 
towards life and your seemingly endless energy. You're truly a power woman!

Wageningen wouldn't have been the same without the wonderful Brazilians with music and sunshine in their souls. André, Cata, Roger, Cris. Thank you for all those beautiful tunes. Maíra, thanks for all the fun times. Renake. You're one of the kind heartiest and caring persons I've ever met. I wish you all the best for your new future. Alexandre. I want to thank you for always having seen my true self.

Thanks to Alba, Pascal and Jenny for the many good moments.

Joost. Our randomness prevented us many time from meeting each other. If we managed, though, I always enjoyed our conversations. You're an intelligent and empathetic person and I wish you to find your path.

I'd like to thank the people from ME and the ISME office. Hans van Veen - for your irresistible laugh that is contagious to everyone who can hear it. Jos, thanks for all your input and support. Paul - thanks for your help and our in-between chats at the coffee machine. Fleur and Mattias - thanks for your bioinformatics support. Annelies - you're a very smart person, conversations with you always trigger my mind. Natalia - Your way of thinking makes me want to sit with you forever and talk about the world. Vivi, thanks for the exchange about volatiles and to work together through the mess of data. Mauricio - thanks for the nice chats. Nori - you have a fantastic way of being and amazing dance moves! Paolo - thanks for sharing many fun moments in the Netherlands and other places in the world. Femke - we always bumped into each other and ended up having great conversations. Thanks for your help for Sweden! Je Seung, you're a very sweet person, thanks for the nice chats. Hans - thanks for measuring countless samples. Saskia, Kees, Maria, Paulien, Marion, Roos - thanks for all your help in the lab. Sabine and Sarash - thanks for all the conversations.

Thank you to my former office mates. Chunken, thanks for the good moments and the time working through the Collimonas genomes. It was not always easy but we managed! Thanks, Irene, for all the in-between conversations over science, traveling and life. Thiago, I really enjoyed our philosophical interludes. Olaf, you're one of the most helpful persons I met during my time at NIOO. Thanks to my present office mates for all the in-between conversations and chocolate in low energy moments.

Thanks to everyone else from the Garbeva group and people from ME! 
Thanks to all other (former) colleagues and friends from NIOO. Veronica, Julie, Kostas, Alexandra, Sabrina, Adrian, Leonardo, Sang Yoon, Michiel, Kadri, Sam, Lysanne, Minghui, Roeland, Sui, Marta Manrubia - You're a kind and caring person and I wish you to find your happiness. Julia - thank you for sharing many common interests and thoughts. Louise, Wim, Arjen, Julie, Veronica, Kadri, Roeland, Stefan, Pella, Koen, Basten, Emilia, Elly, Nurmi, Ana, Kelly, Sigrid, Haikun, Viola, Rutger, Freddy, Andrielli, Ciska, Roel, Tanja, Carolin, Michiel, Tania, Kim, Antica, Sven, Thijs, Annette, Jan, Lukas, Mandy, Peiyu, Suzanne, Thomas, Lucia, Gerda, Froukje, Perro and Carel.

I'd also like to thank my students, Lisette, Dominika, Harm and Jacco, who helped me a lot in my project.

Dani. Danke für all deine Post, die meine halbes Zimmer ausfüllt. Du hast mich immer an deinem Leben teilhaben lassen, auch wenn wir weit voneinander entfernt leben. Du bist ein toller Mensch mit unerschöpflicher Energie und Positivität. Ich bin froh, dich in meinem Leben zu haben!

Maria. Wir sind Freundinnen seitdem ich nach Österreich gezogen bin. Egal wie schwer manche Momente waren, du warst immer für mich da. Dafür danke ich dir von ganzem Herzen. Du bist eine tolle, starke Frau, die immer eine Inspiration für mich war und bleiben wird!

Heike. Du bist die große Schwester, die ich nie hatte. Ich bin dir zutiefst dankbar, dass du mir geholfen hast meinen Weg zu finden und mich an der Arbeit an mir selbst zu unterstützten. Du bist ein sensibler und begabter Mensch, aus dessen Kreativität auch gemeinsame Projekte entstanden sind. Danke, dass du für mich da bist! Walter. Bedankt voor de goede conversaties en muziek.

Liebe Eltern. Ihr habt mich immer in meinen Entscheidungen unterstützt, wenn auch kritisch hinterfragt. Aber so wie ihr mich kennt, hat sich mein Sturkopf dann doch durchgesetzt. Ich bin euch dankbar, dass ihr immer für mich da seid, auch wenn es mich weiter in die Welt zieht. Papa, ich wünsche dir dass du innere Ruhe findest. Mama, du bist ein sehr empathischer und hilfsbereiter Mensch. Vergiss nicht für deine eigenen Bedürfnisse einzustehen. Stephan, Christina, Jael, Christoph und Carmen. Danke dafür, dass ihr für mich da seid und mich immer unterstützt habt. Ich wünsche euch, dass eure Träume in Erfüllung gehen. 



\section{Curriculum vitae}

Ruth Lydia Schmidt was born in Bamberg, Germany on December $20^{\text {th }} 1987$. With the age of 12 she moved to the south of Austria. Tough at first, she managed to find her way through High School and finally graduated with specialization in the Arts and Natural Sciences. Ever since she was fascinated by both disciplines. As there was no specialization for both fields, she chose to go for Natural Sciences and studied Molecular Biology and Biotechnology as part of a trans-disciplinary study between the Karl-Franzens University and Technical University of Graz in Austria.

During one lecture, she heard about the importance of microbes for the environment and about new methods to target those tiny creatures. She contacted the Professor for Environmental Biotechnology, Gabriele Berg, and asked for a project. It brought her to the desert in Egypt, where she studied the effect of microbial inoculants on the native microbial community of Chamomile plants. It was during the time when political turbulences of the Arab Spring was shaking the society of Egypt and didn't leave the work and life unaffected. This time was undoubtedly one of the most influencing and mind-opening experiences in her life so far and ever since she was getting involved in political activities to stand up against discrimination and inequality issues.

In 2013 she started a PhD in microbial ecology at the Netherlands Institute of Ecology in Wageningen. Here, she continued her work on the communication between microorganisms. Throughout her career she missed the link to the Art world. As luck would have it, several projects came along which allowed her to live out her passion for Art aside from her PhD. As an example, the KNAW started a workshop to bring people from different science and art disciplines together and she got to know musicians and a composer with whom she worked on an transdisciplinary project to translate the language of microbes into music.

With this music playing in her head she is now looking for new opportunities in science. 


\section{PE\&RC Training and Education Statement}

With the training and education activities listed below the PhD candidate has complied with the requirements set by the C.T. de Wit Graduate School for Production Ecology and Resource Conservation (PE\&RC) which comprises of a minimum total of 32 ECTS (= 22 weeks of activities)

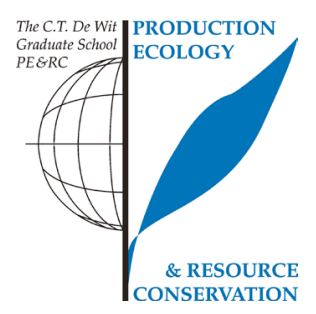

\section{Review of literature (4.5 ECTS)}

Competitive strategies of soil bacteria

\section{Writing of project proposal (4.5 ECTS)}

- Fungal-bacterial interactions: the ability of bacteria to sense fungi (2013)

\section{Post-graduate courses (7.8 ECTS)}

- Introduction to R for statistical analysis; Wageningen, NL (2013)

- Introduction to next generation sequencing; technologies, applications and data analysis; Breda, NL

- IGS Genomics workshop; Baltimore, USA (2013)

- Proteomics summer school; Greifswald, Germany (2013)

- Comparative genomics: from evolution to function; Amsterdam, NL

- Microbial ecology; Soest, NL (2015)

\section{Laboratory training and working visits (9 ECTS)}

- Proteomic responses of bacteria to fungal volatiles; Institute of Microbiology, Greifswald, Germany (2015)

- Fungal volatiles: from gene to product; FEMS research grant; Institute of Mycology and Plant Pathology, Uppsala, Sweden (2016)

\section{Invited review of (unpublished) journal manuscript (9 ECTS)}

- FEMS Microbiology letters: topics in 2013 (2 times), 2014 (2 times), 2015, 2016 (3 times) 
- Applied Microbiology and Biotechnology (2016)

\section{Deficiency, refresh, brush-up courses (4 ECTS)}

- Analytical methods in organic chemistry; Wageningen, NL (2013)

- Ecological models and data in R; PE\&RC, Wageningen, NL (2014)

\section{Competence strengthening / skills courses (8.4 ECTS)}

- PhD Competence assessment; WGS (2013)

- Presentations skills; WGS, Wageningen, NL (2014)

- Academy honours programme for young artists and scientists; The young Academy, Wageningen, NL $(2015,2016)$

- Scientific writing; WGS (2016)

- $\quad$ Making videos for scientists; NIOO, Wageningen, NL (2016)

- Writing for impact; NIOO, Wageningen, NL (2016)

- $\quad$ Career outside university; Uppsala, Sweden (2016)

- Writing grant proposals; WGS (2017)

- Scientific integrity; NIOO, Wageningen, NL (2017)

- Career perspectives; WGS, Wageningen, NL (2017)

\section{PE\&RC Annual meetings, seminars and the PE\&RC weekend (1.2 ECTS)}

- Netherlands Annual Ecology Meeting, NAEM (2013)

- PE\&RC Day (2015)

- Current themes in ecology (2015)

\section{Discussion groups / local seminars / other scientific meetings (20.5 ECTS)}

- Science days; NIOO $(2013,2015)$

- Microbial ecology seminar; NIOO (2013-2017)

- Working group Soilborne pathogens and soil microbiology; NIOO (20132017)

- Soil quality colloquium; NIOO (2013-2017) 
- Microbial ecology PhD group meeting; NIOO (2014-2017)

- Weekly seminars at Department of Forest Mycology and Plant Pathology; Uppsala, Sweden (2016)

- Journal club Department of Forest Mycology and Plant Pathology; Uppsala, Sweden (2016)

\section{International symposia, workshops and conferences (11.7 ECTS)}

- Boston Bacterial Meeting, BBM, Boston, USA (2013)

- CNRS-Jacques Monod conference: bacterial -fungal interactions: a federative field for fundamental and applied microbiology; Roscoff, France (2013)

- $\quad$ 4th International student conference on Microbial Communication MiCom; Jena, Germany (2014)

- Proteomics workshop; Wageningen, NL (2015)

- Ecology of soil microorganisms; Prague, Czech Republic (2015)

- Volatile workshop; Wageningen, NL (2016)

- ISME 16; Montreal, Canada (2016)

\section{Lecturing / supervision of practicals / tutorials}

- Internship students (2015-2017)

\section{Supervision of a MSc student}

- From genes to product: fungal genomics 

The research described in this thesis was carried out at the Department of Microbial Ecology of the Netherlands Institute of Ecology (NIOO-KNAW) in Wageningen.

The project was funded by The Netherlands Organization for Scientific Research (NWO) VIDI personal grant to Paolina Garbeva (864.11.015).

Cover illustration: (c) Heike Engel - 21Lux photography / www.21lux.nl

Design: Ruth Schmidt and Heike Engel

Portrait: (c) Heike Engel

Printing: GVO / www.gvo.nl

This is NIOO thesis number 147 